\title{
Driver Comprehension of Integrated Collision Avoidance System Alerts Presented through a Haptic Driver Seat
}

\author{
Gregory M. Fitch \\ Dissertation submitted to the faculty of the \\ Virginia Polytechnic Institute and State University \\ in partial fulfillment of the requirements for the degree of
}

DOCTOR OF PHILOSOPHY

In

Industrial and Systems Engineering

Dr. Brian M. Kleiner, Chair

Dr. Thomas A. Dingus

Dr. Jonathan M. Hankey

Dr. Woodrow W. Winchester

December $9^{\text {th }}, 2008$

Blacksburg, Virginia

Keywords: Active Safety, Alert, Collision Avoidance, Collision Avoidance System, Collision Warning, Crash Alert, Integrated Collision Avoidance System, Haptic, Haptic Driver Seat, Highway Safety, Surface Transportation, Tactile, Vibration, Vibrotactile 


\title{
Driver Comprehension of Integrated Collision Avoidance System Alerts
}

Presented through a Haptic Driver Seat

\author{
Gregory M. Fitch
}

\begin{abstract}
Active safety systems that warn automobile drivers of various types of impending collisions have been developed. How these systems alert drivers when integrated, however, is a crucial component to their effectiveness that hinges on the consideration of human factors. Drivers' ability to comprehend multiple alerts presented through a haptic driver seat was investigated in this dissertation. Twenty-four participants, balanced for age and gender, drove an instrumented vehicle on a test-track while haptic alerts (vibrations in the driver seat) were generated. Drivers' ability to transmit the information conveyed by the alerts was investigated through two experiments. The first experiment investigated the effects of increasing the number of potential alerts on drivers' response performance. The second experiment investigated whether presenting haptic alerts through unique versus common locations in the driver seat affects drivers' response performance. Younger drivers (between the ages of 18 and 25 years old) were found to efficiently process the increased information contained in the alerts, while older drivers were not as efficient. However, it is foreseeable that older driver performance decrements may be assuaged when a crash context is provided. A third experiment evaluated the haptic driver seat's ability to alert distracted drivers to an actual crash threat. Drivers that received a haptic seat alert returned their gaze to the forward roadway sooner, removed their foot from the throttle sooner, pressed the brake pedal sooner, and stopped farther away from an inflatable barricade than drivers that did not receive a haptic seat alert. No age or gender effects were found in this experiment. Furthermore, half of the drivers that received the haptic seat alert lifted up on the throttle before returning their eyes to the forward roadway. This suggests these drivers developed an automatic response to the haptic seat alerts through their experience with the previous two experiments. A three-alert haptic seat approach, the intermediate alternative tested, is recommended providing specific design requirements are met.
\end{abstract}




\section{DEDICATION}

This dissertation is dedicated to my fiancée Elizabeth, my grandparents Joyce, Francine, and Andre, my parents Stephen and Monique, as well as my brother Jeff and my sister Stephanie. Thank you for your tremendous support. 


\section{ACKNOWLEDGEMENTS}

I would first like to thank my committee Dr. Brian Kleiner, Dr. Tom Dingus, Dr. Jon Hankey, and Dr. Woodrow Winchester for their invaluable feedback. I have become a better researcher through their instruction. I would like to thank Dr. Raymond Kiefer and Donald Grimm from General Motors Corporation for providing the Inseat Solutions, LLC. inertial shaker tactors and programming logic. Jared Bryson and Fang Huang are acknowledged for helping me with the hardware and software development. I would also like to thank Amy Wharton, Rory Brannan, and Julia Dyman for assisting me with my research. Last, but not least, I would like to thank my sister Stephanie for proof reading this document.

Human Factors has been a passion of mine ever since I learned about designing systems to match humans' abilities and limitations in my sophomore year of Industrial Engineering at the University of Toronto. Over the past decade, many colleagues have provided opportunities for me to study and practice Human Factors engineering. I would like to thank them for their benevolence. 


\section{TABLE OF CONTENTS}

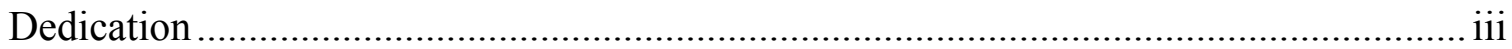

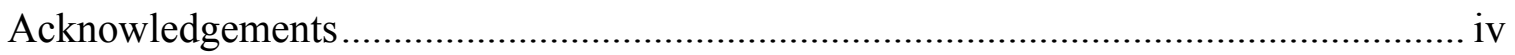

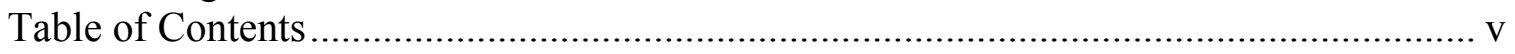

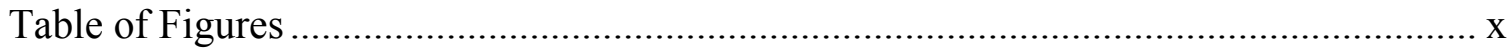

Table of Tables ................................................................................................ xiv

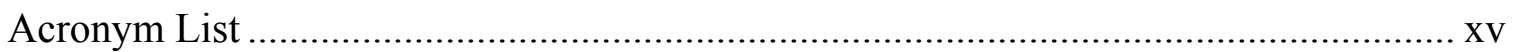

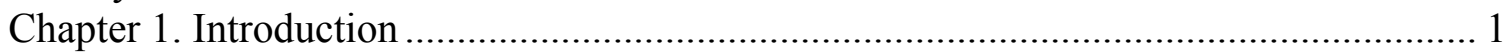

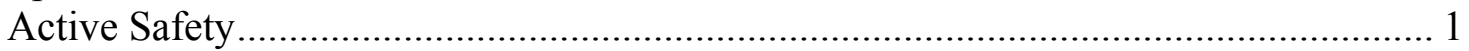

Why Alert Drivers?

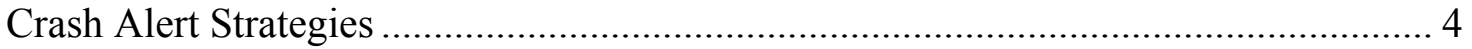

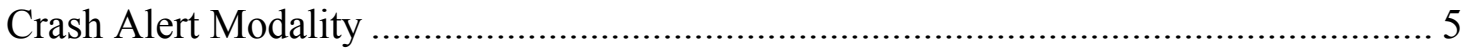

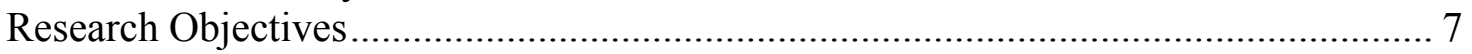

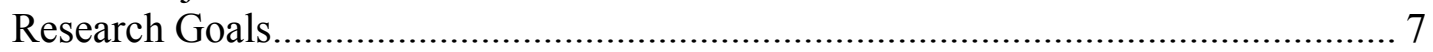

Research Questions ................................................................................... 7

Research Question 1: What is the effect of increasing the number of CAS alerts on driver performance? ................................................................................. 7

Research Question 2: What is the effect of the haptic seat alert location on drivers' ability to differentiate meaningful from uninformative alerts? ................. 8

Research Question 3: What is the effect of a haptic seat alert on drivers' collision

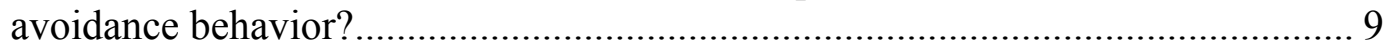

Chapter 2. Literature Review .............................................................................. 10

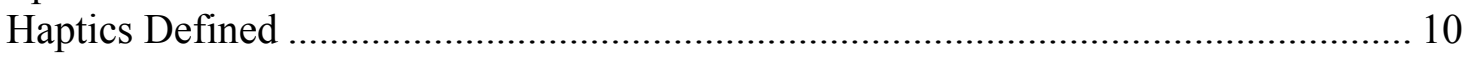

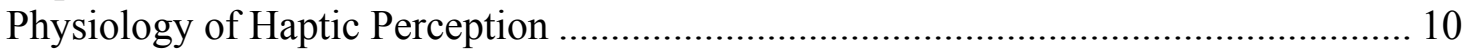

Factors Affecting Perception of Vibration............................................................ 10

Types of Mechanoreceptors ........................................................................ 10

Number, Distribution, and Placement of Mechanoreceptors .................................. 13

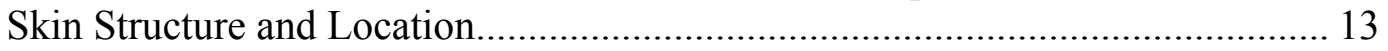

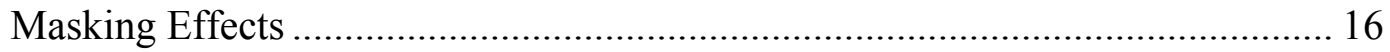

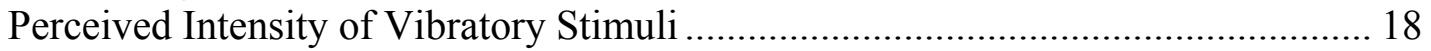

Contours of Haptic Sensation ....................................................................... 18

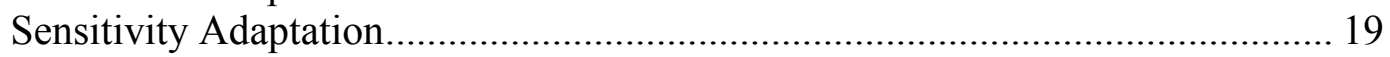

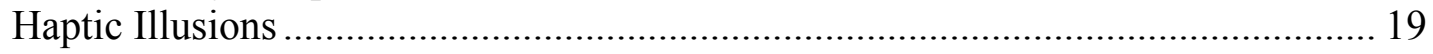

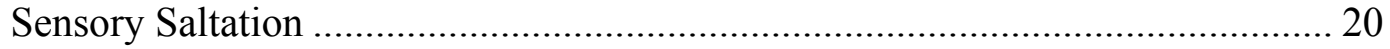

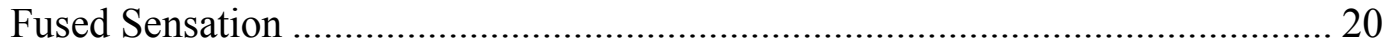

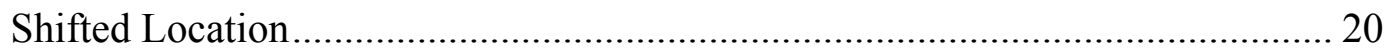

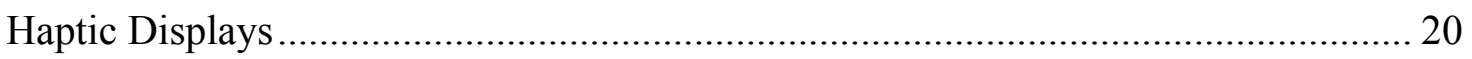

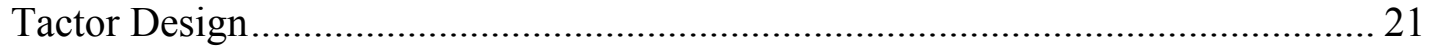

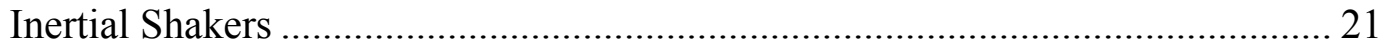

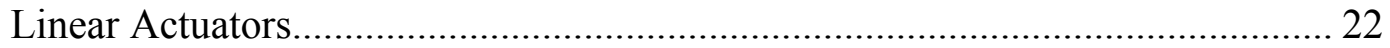

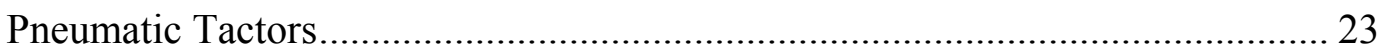

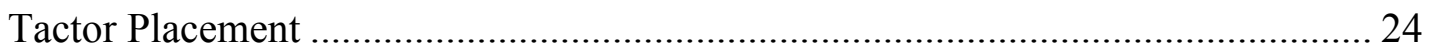

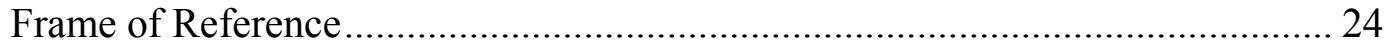

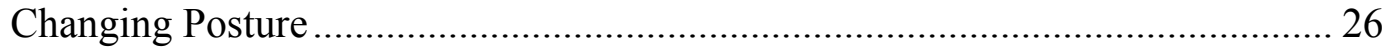

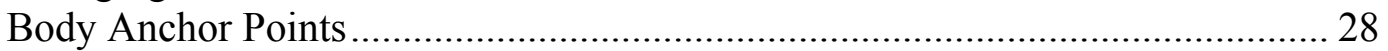




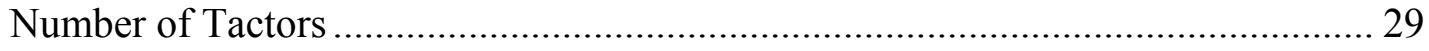

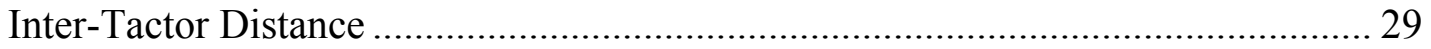

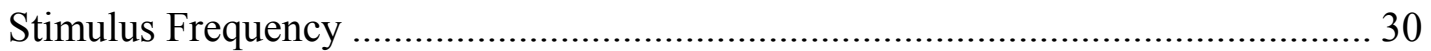

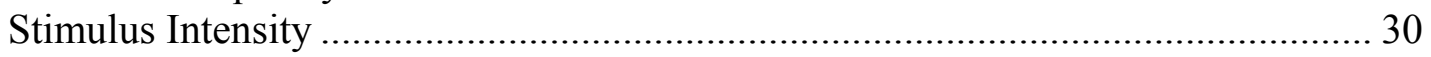

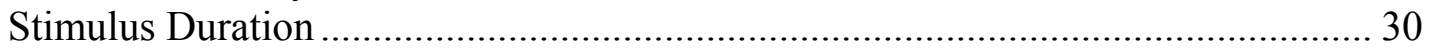

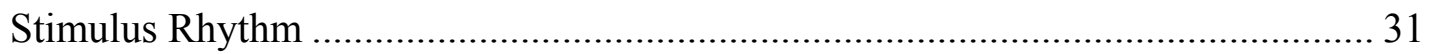

Haptic Display Design Constraints ........................................................................ 31

Chapter 3. Experiment 1: Effects of Increasing the Number of CAS Alerts on Driver

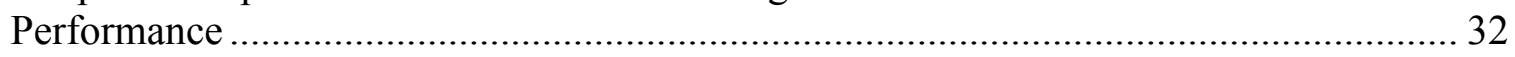

Purpose

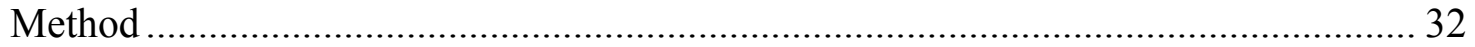

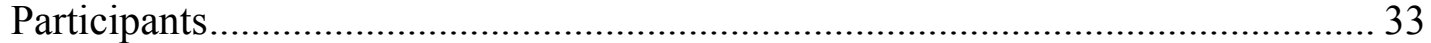

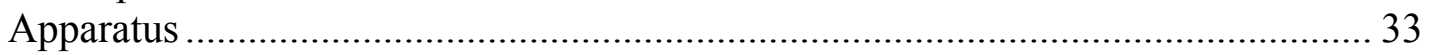

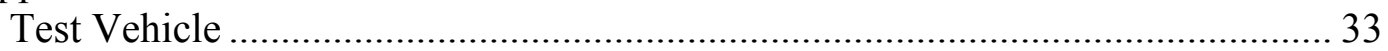

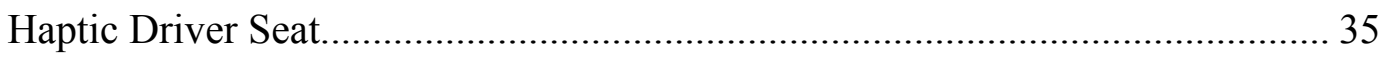

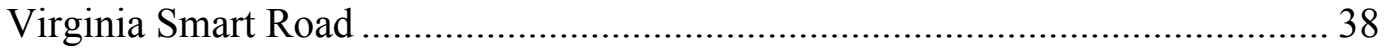

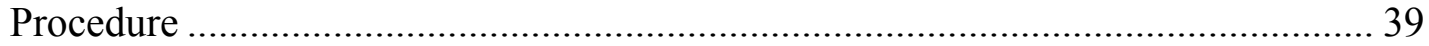

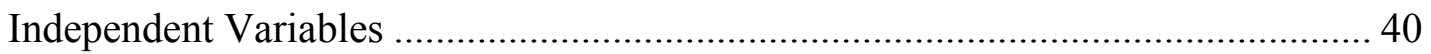

Age (Older Younger) - Between-Subjects ........................................................... 40

Gender (Female, Male) - Between-Subjects .......................................................... 41

Number of Alerts (One Alert, Three Alerts, Seven Alerts) - Within-Subjects .... 41

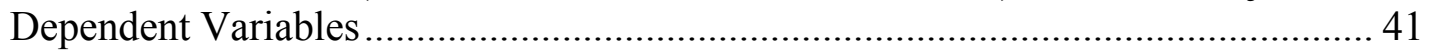

Manual Response Accuracy............................................................................ 41

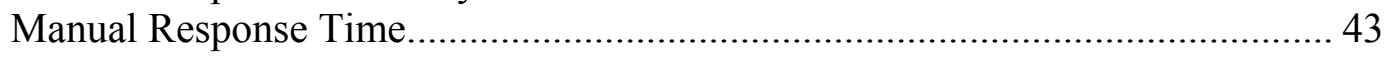

Verbal Response Accuracy ......................................................................... 43

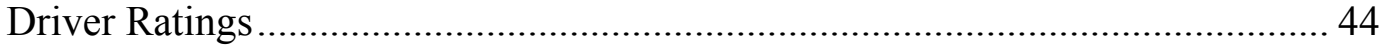

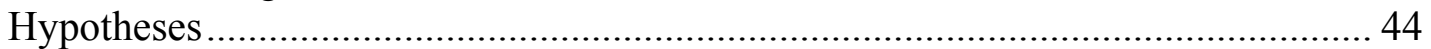

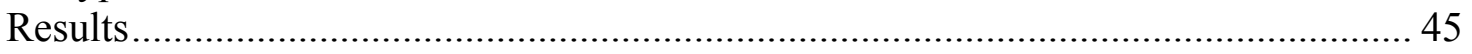

Manual Response Accuracy............................................................................... 45

Practical Manual Response Accuracy ............................................................... 45

Theoretical Manual Response Accuracy ........................................................ 47

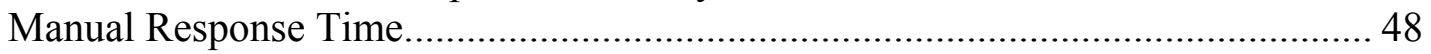

Practical Manual Response Time................................................................. 48

Hick-Hyman Law of Choice Response Time .................................................... 52

Theoretical Manual Response Time …………………..................................... 54

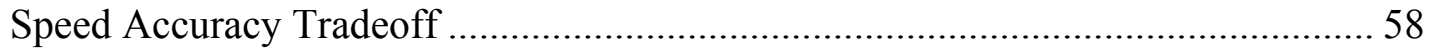

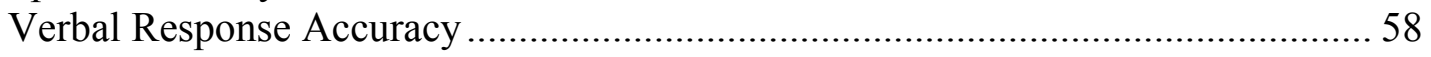

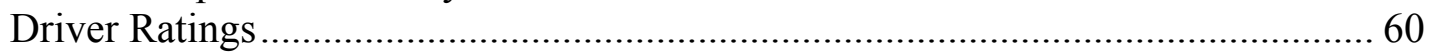

Question 1: Please rate how difficult it was to make the correct driving action for

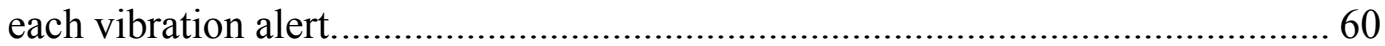

Question 2: Please rate how difficult it was to identify the vibration alerts......... 62

Question 3: Please rate how confusing the vibration alerts were. ........................ 63

Question 4: Please rate how difficult it was to perform the task in Experiment 1.

Question 5: Please rate how distinct the vibration alerts were from each other... 65 
Question 6: Please rate how appropriate it was for the seat to vibrate like this

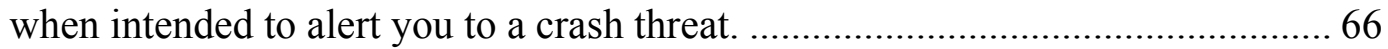

Discussion

Chapter 4. Experiment 2: Effects of Haptic Seat Alert Location on Drivers' Ability to

Differentiate Meaningful from Uninformative Alerts ............................................ 71

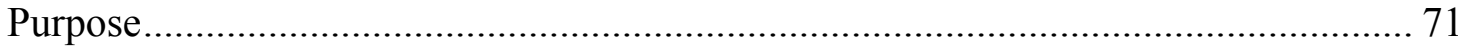

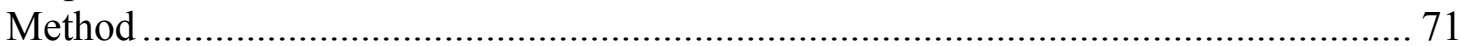

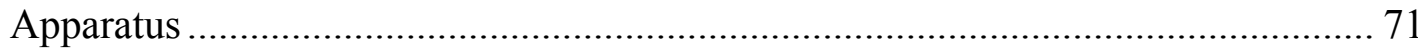

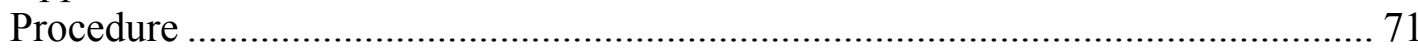

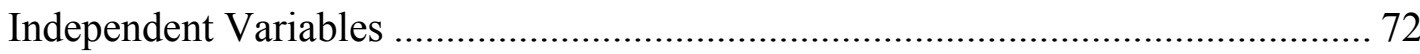

Age (Older, Younger) - Between-Subjects ..................................................... 72

Gender (Female, Male) - Between-Subjects …........................................... 72

Side (Left, Right) - Within-Subjects ........................................................ 72

Location (Same Location, Different Location) - Within-Subjects...................... 72

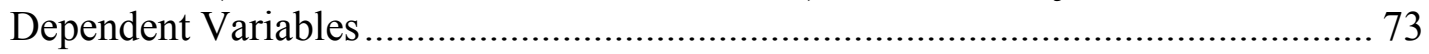

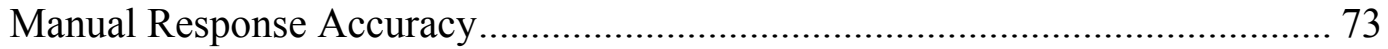

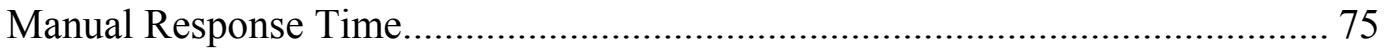

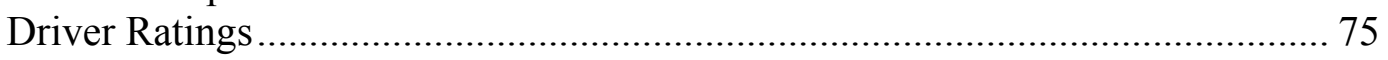

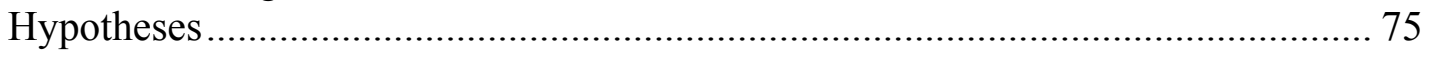

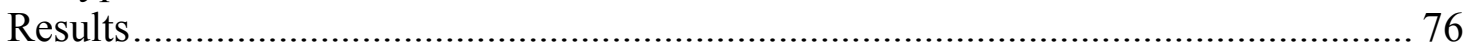

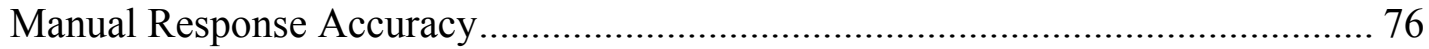

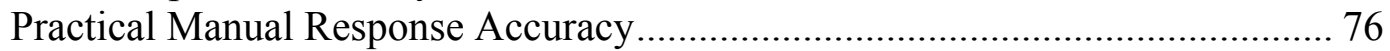

Theoretical Manual Response Accuracy ....................................................... 78

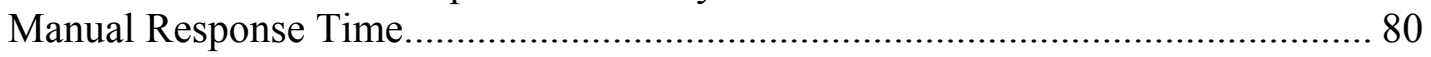

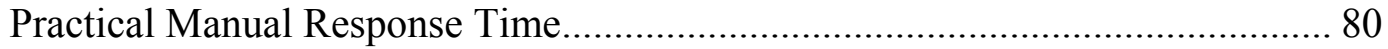

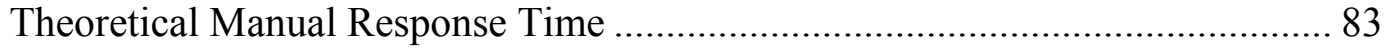

Speed Accuracy Tradeoff Inspection ............................................................... 86

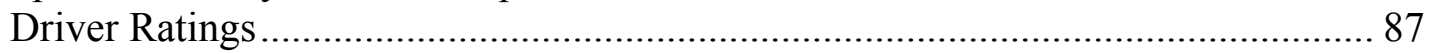

Question 1: Please rate how easy it was to distinguish meaningful vibration alerts from those you were not supposed to respond to......................................... 87

Question 2: Please rate how distinct the vibration alerts were from each other... 88 Question 3: Please rate how difficult it was to perform the task in Experiment 2.

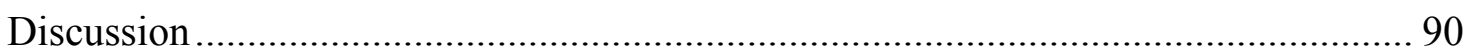

Chapter 5. Experiment 3: Effects of a Haptic Seat Alert on Driver Collision Avoidance

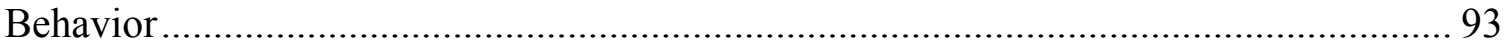

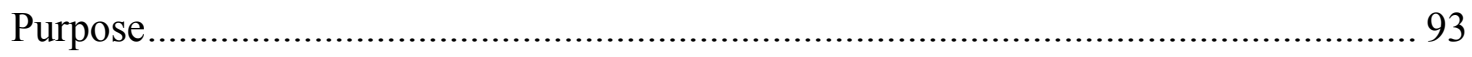

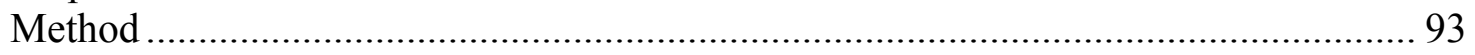

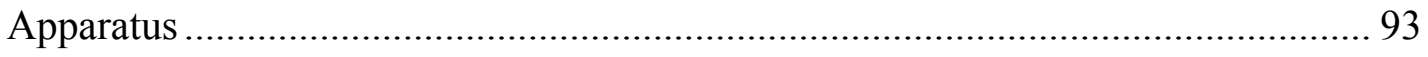

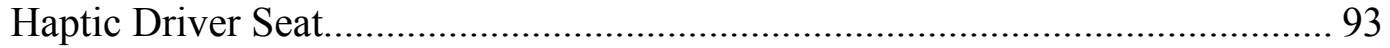

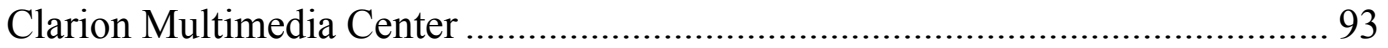

The Inflatable Barricade ............................................................................ 94

Differential Global Positioning System (DGPS) .............................................. 95

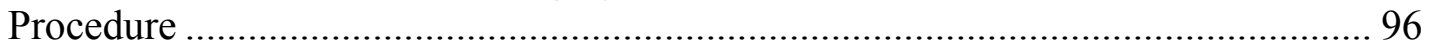

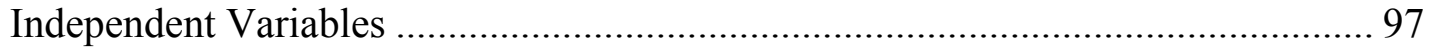

Age (Older, Younger) - Between-Subjects ............................................... 97 
Gender (Female, Male) - Between-Subjects ............................................................ 97

Alert (On, Off) - Between-Subjects …………….............................................. 97

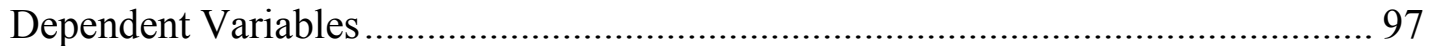

Collision with Barricade ................................................................................... 98

Time from Inflatable Barricade Activation to Returning Eyes to the Forward

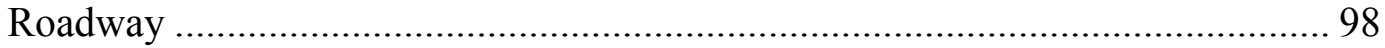

Foot-Off Throttle Response Time.................................................................... 98

Foot-On Brake Response Time..................................................................... 98

Number of Drivers that Pressed the Brake Pedal Prior to Returning their Gaze to

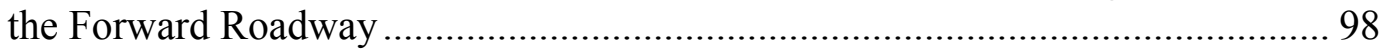

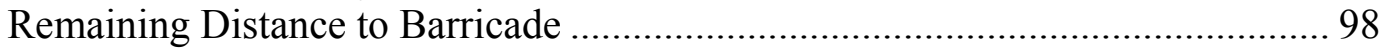

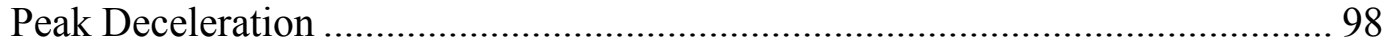

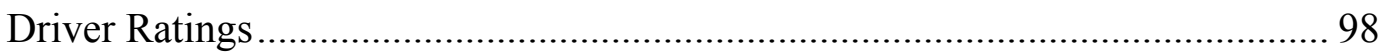

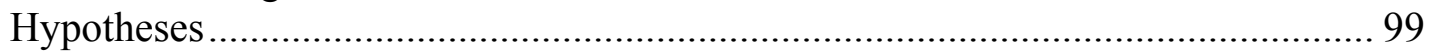

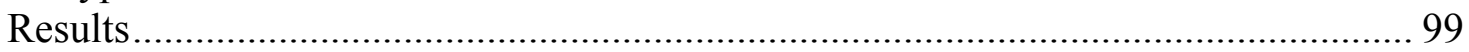

Collision with Barricade ................................................................................... 99

Time from Barricade Inflating to Driver Returning Eyes to the Forward Roadway 99

Foot-Off Throttle Response Time................................................................... 100

Foot-On Brake Response Time............................................................................ 101

Number of Drivers that Pressed the Brake Pedal Prior to Returning Gaze to the

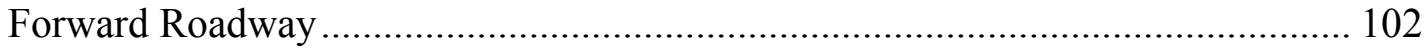

Remaining Distance to Barricade .................................................................... 102

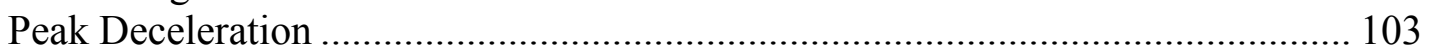

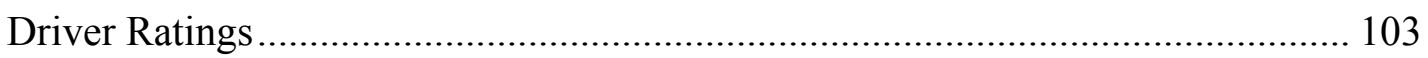

Question 1: Please rate how distracted you were while the barricade was inflated.

Question 2: Please rate how surprised you were that you had to stop............... 104

Question 3: Please indicate the degree to which the vibration made you look forward using the options listed below ........................................................... 105

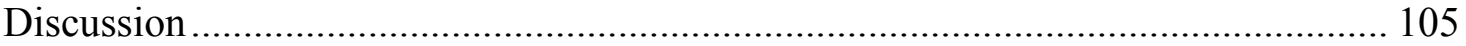

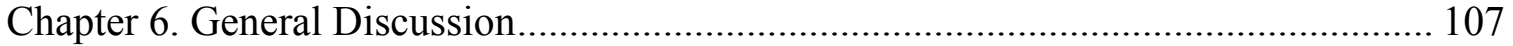

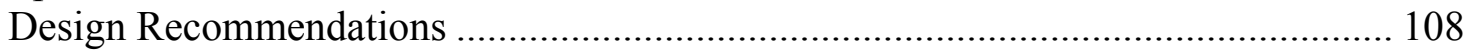

1) High Degree of Stimulus-Response Compatibility ................................... 108

2) Consideration for Drivers' Frame of Reference ........................................... 109

3) Unique Tactors for Each Alert................................................................ 109

4) Large Distance between Tactors............................................................... 109

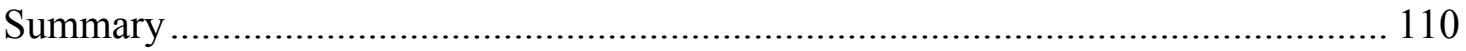

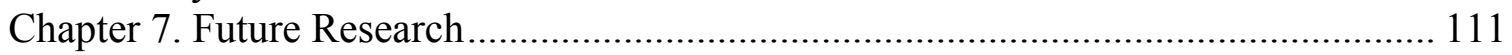

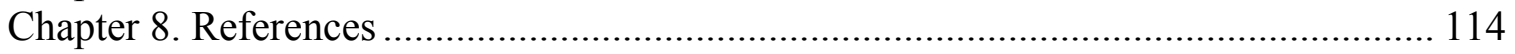

Appendix A - Telephone Script and Driver Screening Questionnaire............................ 119

Appendix B - Virginia Smart Road..................................................................... 126

Weather-Making Capability................................................................................... 126

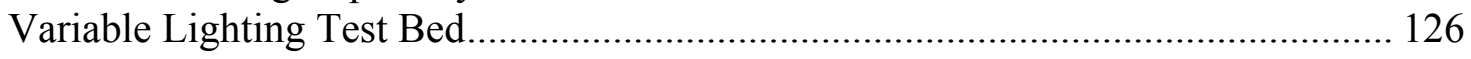

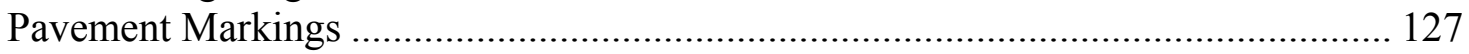

On-Site Data Acquisition and Road Weather Information Systems........................... 127

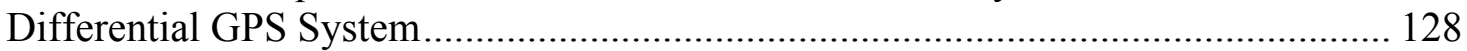


Road Access and Surveillance ............................................................................ 128

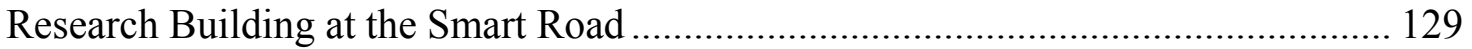

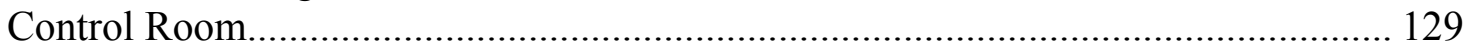

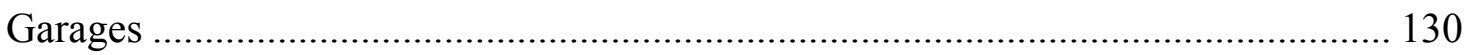

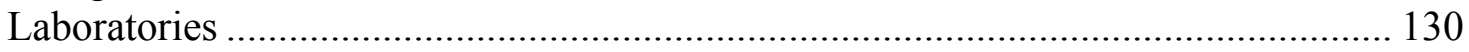

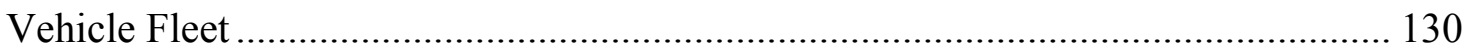

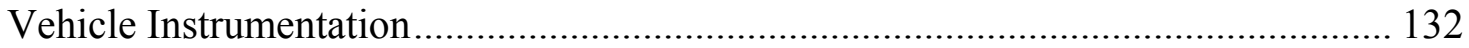

Appendix C - Informed Consent Form ............................................................... 133

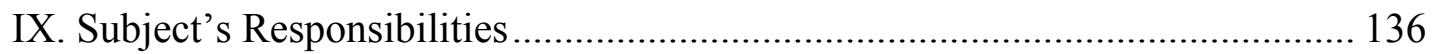

X. Consent to Use Video/Audio Data for Research Reporting Purposes ............... 136

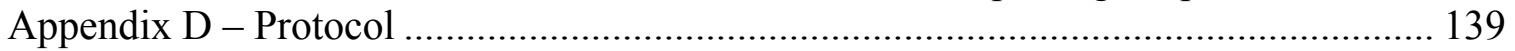

Appendix E - Number of Alerts Questionnaire ....................................................... 162

Appendix F - Number of Alerts Questionnaire (Overall) ............................................. 164

Appendix G - Removal of Response Time Outliers ..................................................... 165

Appendix H - Additional Driver Ratings of Alerts in Experiment 1 ............................. 166

Appendix I - Haptic Alert Location Questionnaire...................................................... 170

Appendix J - Haptic Alert Location Questionnaire (Overall)......................................... 171

Appendix K - Additional Driver Ratings of Alerts in Experiment 2 ............................ 173

Appendix L - Surprise Braking Event ...................................................................... 179

Appendix M - Surprise Braking With Haptic Seat ........................................................... 181

Appendix N - Scatter Plots of the Variables Analyzed in Experiment 3 ...................... 184

Time from Inflatable Barricade Activation to Returning Eyes to the Forward

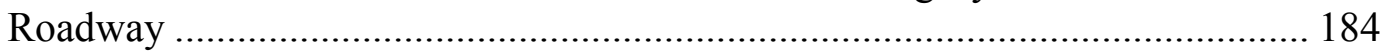

Foot-Off Throttle Response Time................................................................ 185

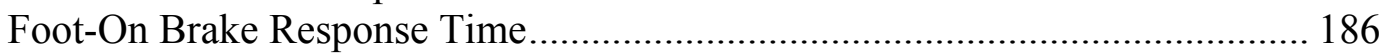

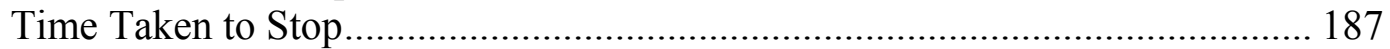

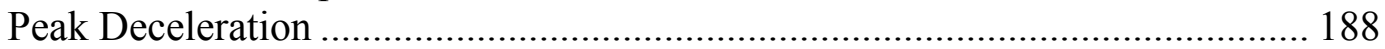

Distance Between Car and Inflatable Barricade …………................................. 189

Appendix O - Experiment 3 Driver Ratings.............................................................. 190 


\section{TABLE OF FIGURES}

Figure 1. Histological depiction of four mechanoreceptors in glabrous skin. Adapted from Greene (2007).

Figure 2. Two-point discrimination thresholds for the human body. Adapted from Greene (2007).

Figure 3. Thresholds for $200 \mathrm{~Hz}$ vibration for one male subject. Adapted from Boff and Lincoln (1988).

Figure 4. Upper: Standard English Braille alphabet. Lower: Standard English Braille alphabet with border. Adapted from Loomis (1983).

Figure 5. Equal subjective intensity curves for vibratory stimuli. Adapted from Boff and Lincoln (1988).

Figure 6. Left side: An eccentric mass tactor. Right side: 64 eccentric mass tactors positioned in a foam driver seat. Reproduced with permission from Fitch, Kiefer, Hankey and Kleiner (2007). Copyright 2007 by the Human Factors and Ergonomics Society. All rights reserved.

Figure 7. A linear actuator tactor.

Figure 8. Four pneumatic tactors attached to a belt. Reproduced with permission from Dr. Roger Cholewiak's website (http://www.princeton.edu/ rcholewi/TRLTactorArrays.html) .....

Figure 9. Average perceived directions in response to 15 tactors mounted around users waists via a belt. Reproduced with permission from Van Erp (2005). Copyright 2005 by Taylor and Francis Group (http://www.informaworld.com). All rights reserved............ 25 Figure 10. Center image: the $8 \times 8$ tactor array embedded in the driver seat pan. Tactors are represented by bold diagonal lines. The target locations are denoted by the dashed line borders. A seat pan pressure distribution plot is also shown (where darker areas indicate increased pressure). Peripheral graphs: the localization scores for each target location (front (F), front-right (FR), right (R), back-right (BR), back (B), back-left (BL), left (L), and front-left (FL)). Note: the front target location was generated by simultaneously activating the FL and FR target locations. Reproduced with permission from Fitch, Kiefer, Kleiner, et al. (2007). Copyright 2007 by the Human Factors and Ergonomics Society. All rights reserved.

Figure 11. The 2002 Cadillac STS test vehicle. Counterclockwise starting from the top:

a) profile photograph of test vehicle, b) the data acquisition system, c) in-vehicle experimenter's computer to control the presentation of the haptic seat alerts................. 34

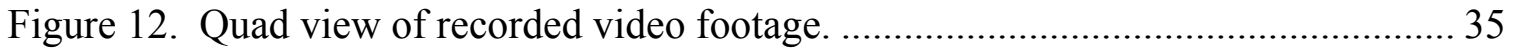

Figure 13. Mounting of the six inertial shaker tactors in the driver seat. ....................... 36

Figure 14. Close-up of the inertial shaker tactors manufactured by InSeat Solutions,

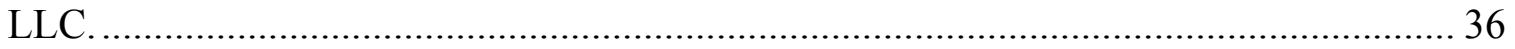

Figure 15. Diagram of the Virginia Smart Road...................................................... 39

Figure 16. Drivers' practical manual response accuracy broken down by the age and gender of the drivers.

Figure 17. Drivers' theoretical manual response accuracy broken down by the age and gender of the drivers.

Figure 18. Histogram of drivers' practical manual response times to one, three, and seven alerts. 
Figure 19. Alert practical manual response time.

Figure 20. Drivers' $90^{\text {th }}$ percentile practical manual response times............................. 51

Figure 21. The Hick-Hyman Law of choice response time as exemplified by drivers' mean response times to an FCW alert presented on its own, with two other alerts, and

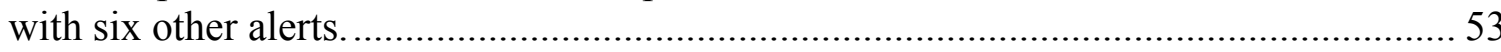

Figure 22. Drivers' practical manual response time to FCW alerts.............................. 54

Figure 23. Histogram of drivers' theoretical manual response time to one, three, and seven alerts.

Figure 24. Drivers' theoretical manual response times by age and gender. ................... 56

Figure 25. Drivers' $90^{\text {th }}$ percentile theoretical manual response times........................... 57

Figure 26. Speed-accuracy tradeoff investigation. For each data series, the smallest symbol represents the speed-accuracy data point associated with one alert, the next largest symbol represents the speed-accuracy data point associated with three alerts, and the largest symbol represents the speed-accuracy data point associated with seven alerts.

Figure 27. Drivers' verbal response accuracy. 58

Figure 28. Drivers' ratings of how difficult it was to make the correct driving action for each vibration alert.

Figure 29. Drivers' mean ratings of how difficult it was to identify the vibration alerts. 62

Figure 30. Drivers' mean ratings of how confusing the alerts were.

Figure 31. Drivers' mean ratings of how difficult it was to perform the manual and verbal response task in Experiment 1.

Figure 32. Drivers' mean rating of how distinct the alerts were.

Figure 33. Drivers' mean ratings of how appropriate it was for the driver seat to vibrate when alerting drivers to a crash threat. 66

Figure 34. Drivers' practical manual response accuracy to alerts presented through just the seat pan, or both the seat pan and seat back.

Figure 35. Drivers' practical manual response accuracy to just the meaningful alerts. .. 78

Figure 36. Drivers' theoretical manual response accuracy broken down by their age and

gender.

Figure 37. Drivers' theoretical manual response accuracy to just the meaningful alerts. 80 Figure 38. Practical manual response times to meaningful alerts presented through the seat pan, as well as through the seat back.

Figure 39. Drivers' mean practical manual response time to meaningful alerts. ........... 82

Figure 40. Drivers' $90^{\text {th }}$ percentile practical manual response times to meaningful alerts.

Figure 41. Histogram of drivers' theoretical manual response time to meaningful alerts presented through the seat pan, as well as through the seat back.

Figure 42. Drivers' mean theoretical manual response times to meaningful alerts......... 85

Figure 43. Drivers' $90^{\text {th }}$ percentile theoretical manual response times to meaningful alerts.

Figure 44. Speed-accuracy tradeoff inspection. The smaller symbols represent the speed-accuracy data points observed when the alerts were presented through the seat pan, while the larger symbols represent the speed-accuracy data points observed when the alerts were presented through the seat back. 
Figure 45. Drivers' mean ratings of how easy it is to distinguish meaningful vibration alerts from those that they are not supposed to respond to....................................... 88 Figure 46. Drivers' ratings of how distinct the vibration alerts were from each other.... 89 Figure 47. Drivers' mean ratings of how easy it is to perform the task in Experiment 2.90 Figure 48. Representation of drivers' judgment of vibrotactile stimuli according to a) one dimension, and b) two correlated separable dimensions. Adapted from Wickens \&

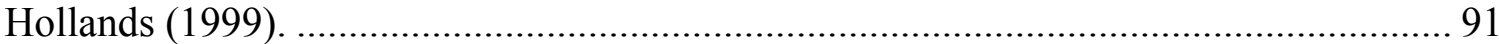

Figure 49. Clarion touch-screen multimedia center................................................... 94 Figure 50. Eight inflatable nylon cylinders that span the Smart Road lanes and right

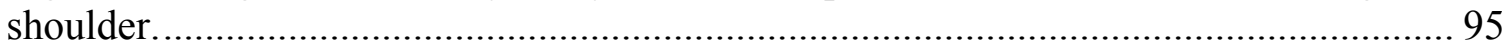
Figure 51. Antenna mounted to trunk of test vehicle that was used for the Differential

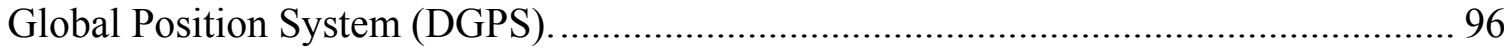
Figure 52. Drivers' mean time to return their gaze to the forward roadway broken down by age and gender.

Figure 53. Drivers' mean time to remove their foot from the throttle broken down by age and gender..... 101

Figure 54. Drivers' mean time to press the brake pedal broken down by age and gender.

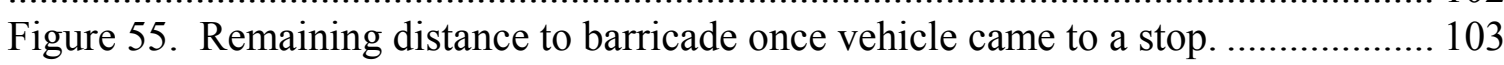

Figure 56. Drivers' ratings of how distracted they were while the barricade inflated... 104

Figure 57. Drivers' ratings of how surprised they were that they had to stop.............. 105

Figure 58. Fog, rain, and snow equipment installed on the Smart Road. ...................... 126 Figure 59. All-weather testing equipment with experimental lighting test bed installed on

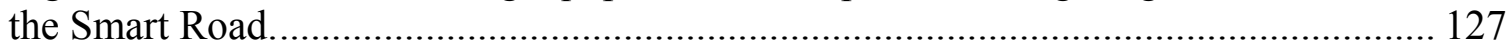

Figure 60. Bunker with DAS and weather station installed on the Smart Road........... 128

Figure 61. Gate that restricts access to the Smart Road............................................. 129

Figure 62. Smart Road control room and dispatcher monitoring research................... 130

Figure 63. VTTI's vehicle fleet on the Smart Road bridge. ...................................... 131

Figure 64. Visual acuity test chart positioning. ....................................................... 144

Figure 65. Scatter plot of the observed practical manual response times for each

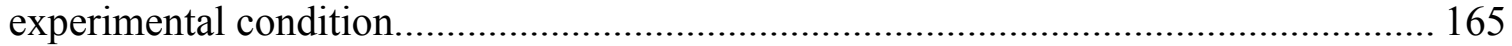

Figure 66. Drivers' mean ratings of how urgent the alerts were. ................................. 166

Figure 67. Drivers' mean ratings of how annoying the alerts were.............................. 167

Figure 68. Drivers' mean ratings of how intense the alerts were. ................................ 168

Figure 69. Drivers' mean ratings of how long the alerts were.................................... 169

Figure 70. Drivers' mean ratings of the intensity of the vibration alerts that were

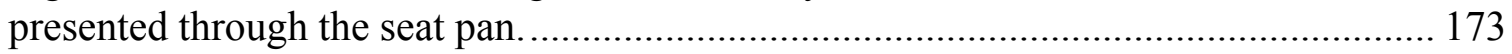

Figure 71. Drivers' mean ratings of the intensity of the vibration alerts that were

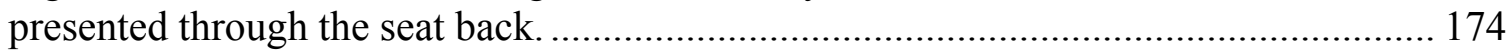

Figure 72. Drivers' mean ratings of how annoying the vibration alerts were when

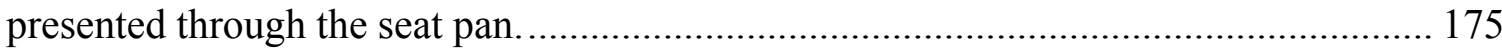

Figure 73. Drivers' mean ratings of how annoying the vibration alerts were when

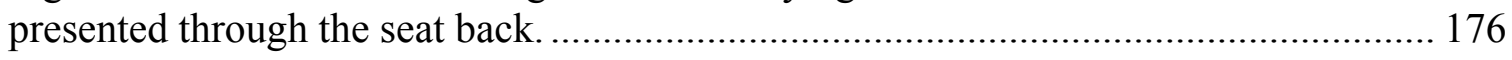
Figure 74. Drivers' mean ratings of how appropriate it is to vibrate the bottom of the driver seat to present an alert. 
Figure 75. Drivers' mean ratings of how appropriate it is to vibrate the back of the driver seat to present an alert...................................................................................... 178

Figure 76. Time from inflatable barricade activation to returning eyes to the forward

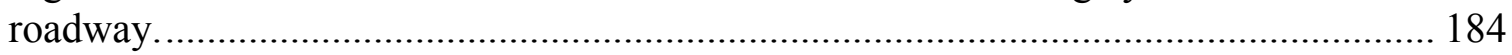

Figure 77. Foot-off throttle response time. ............................................................ 185

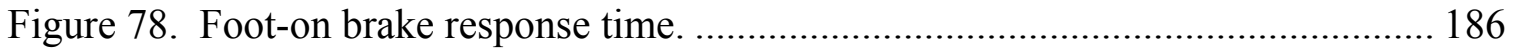

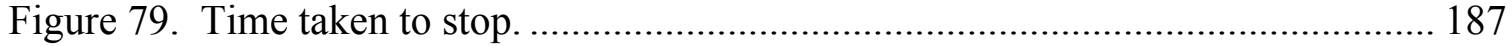

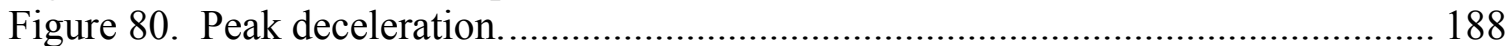

Figure 81. Remaining distance between vehicle and inflatable barricade.................... 189

Figure 82. Drivers' rating of event's similarity to an actual emergency braking event. 190

Figure 83. Drivers' ratings of how fast they pressed the brakes broken down by age and

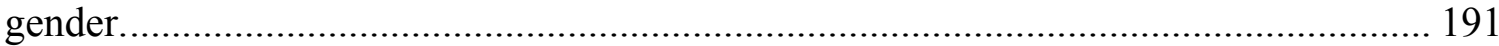

Figure 84. Drivers' ratings of how hard they pressed on the brakes broken down by age

and gender. 192

Figure 85. Drivers' ratings of how well the vibrating seat grabbed their attention broken

down by age and gender.

Figure 86. Drivers' ratings of how appropriate the vibrating seat was for telling them to look forward broken down by age and gender. 194

Figure 87. Drivers' ratings of how much they would like their driver seat to vibrate to warn them of an impending collision broken down by age and gender.

Unless otherwise stated, all images are the property of the author. 


\section{TABLE OF TABLES}

Table 1. List of Automotive Collision Avoidance Systems (CASs) (SAE/Automotive

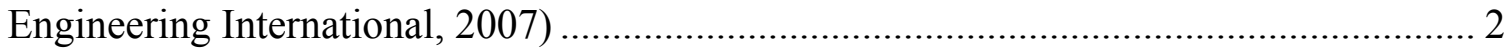

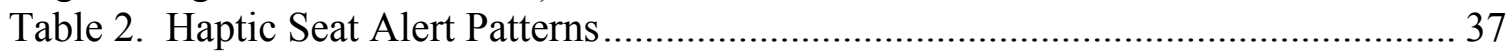

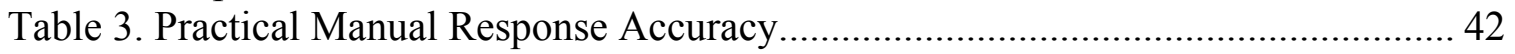

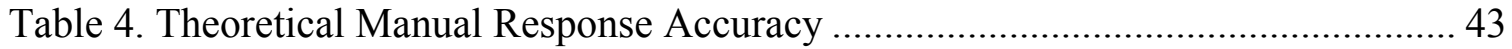

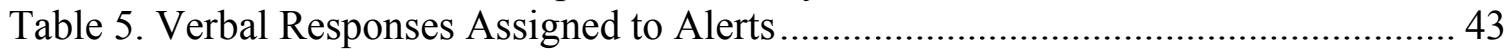

Table 6. Drivers' Practical Response Time Quantiles by Number of Alerts.................. 51

Table 7. Computation of Information Conveyed by an FCW Alert .............................. 52

Table 8. Drivers' Theoretical Response Time Quantiles by Number of Alerts............... 57

Table 9. Changes in Drivers' Mean Practical Manual Response Times......................... 68

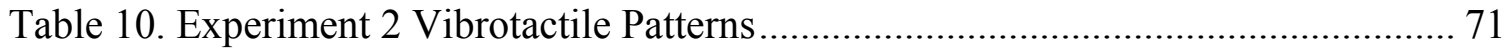

Table 11. Permitted Maneuvers for the Practical Manual Accuracy Score ...................... 74

Table 12. Permitted Maneuvers for the Theoretical Manual Accuracy Score................ 75

Table 13. Quantiles of Drivers' Manual Response Time to Meaningful Alerts ............. 83

Table 14. Quantiles of Drivers' Theoretical Manual Response Times.......................... 85

Table 15. Experiment 3 Vibrotactile Seat Alert Pattern ............................................. 93 


\section{ACRONYM LIST}

\begin{tabular}{|c|c|}
\hline CAS & Collision Avoidance System \\
\hline $\mathrm{CNS}$ & Central Nervous System \\
\hline CSW & Curve Speed Warning \\
\hline $\mathrm{C} / \mathrm{VIS}$ & Camera/Video Imaging System \\
\hline $\mathrm{dB}$ & Decibel \\
\hline DGPS & Differential Global Positioning Satellite \\
\hline ESC & Electronic Stability Control \\
\hline FA & Fast Adapting \\
\hline FCW & Forward Collision Warning \\
\hline HVAC & Heating Ventilation and Air Conditioning \\
\hline IVW & Intersection Violation Warning \\
\hline ITS & Intelligent Transportation Systems \\
\hline JND & Just Noticeable Difference \\
\hline $\mathrm{LCW}$ & Lane Change Warning \\
\hline LDW & Lane Departure Warning \\
\hline LED & Light-emitting diode \\
\hline NHTSA & National Highway Traffic Safety Administration \\
\hline RE & Rear-End \\
\hline SA & Slow Adapting \\
\hline TTC & Time-to-collision \\
\hline
\end{tabular}




\section{CHAPTER 1. INTRODUCTION}

In the event of a collision, substantial forces are placed on the vehicle's occupants. To compare, the driver of a vehicle crashing into a telephone pole at $72 \mathrm{~km} / \mathrm{h}$ (45 mph) collides with the windshield with the same force as falling to the ground from a five story building. Automotive engineering over the past four decades has improved vehicles' ability to absorb crash energy before it is transferred to the vehicle occupants. A vehicle's ability to prevent injury to its occupants during a collision is termed its crashworthiness (National Highway and Traffic Safety Administration, 2007a).

Automotive crashworthiness has greatly improved through the use of seat belts, advanced front and side air bags, crumple zones, side impact beams, and reinforced roofs (National Highway and Traffic Safety Administration, 2007a). Yet, there are still over 43,000 automotive crash fatalities per year (National Highway and Traffic Safety Administration, 2007b). It is believed that developments in passive safety measures have reached saturation (SAE/Automotive Engineering International, 2007). Broader issues, such as driver distraction, impairment, as well as an exponential increase in the number of vehicles in the roadway infrastructure, have led automotive engineers to look for alternative approaches to saving lives.

\section{ACTIVE SAFETY}

Greater return-on-investments are anticipated from systems that prevent collisions from occurring in the first place (SAE/Automotive Engineering International, 2007). This active safety approach evaluates the crash threat of surrounding objects and notifies the driver when they become threatening. Various active safety systems have resulted from the nexus of transportation, computing, and communication (SAE/Automotive Engineering International, 2007). Controller area networks allow computers to monitor and electronically control a vehicle's components. For instance, a mandated safety feature by the National Highway Traffic Safety Administration (NHTSA) is electronic stability control (ESC). ESC enhances directional control and the stability of the vehicle in various conditions. ESC uses sensors in the vehicle to determine the vehicle path intended by the driver and compares it to the actual path of the vehicle. When the actual path does not meet the intended path, ESC applies the brake of the appropriate wheel to assist in counteracting the oversteer or understeer condition (DaimlerChrysler Corporation, 2007). This safety feature alone is expected to significantly reduce the number fatalities resulting from single lane road departures (SAE/Automotive Engineering International, 2007). Safety systems such as ESC become increasingly effective at preventing collisions through inter-vehicle communication. For instance, by notifying trailing vehicles when a lead vehicle slides off an icy road, not only can drivers respond by slowing down (after being notified by an alert), but their vehicles can prepare for the changing conditions (e.g., by engaging all-wheel drive). The combined drivervehicle response can decrease the likelihood of skidding off the road. Table 1 lists other collision avoidance systems (CASs) and driver assist systems that are designed to help drivers avoid crash threats. 
Table 1. List of Automotive Collision Avoidance Systems (CASs) (SAE/Automotive Engineering International, 2007)

\begin{tabular}{|c|c|}
\hline System & Purpose \\
\hline $\begin{array}{l}\text { Forward Collision Warning } \\
(\mathrm{FCW})\end{array}$ & $\begin{array}{l}\text { Notify drivers of an impending rear-end } \\
\text { (RE) collision }\end{array}$ \\
\hline $\begin{array}{l}\text { Intersection Violation Warning } \\
\text { (IVW) }\end{array}$ & $\begin{array}{l}\text { Notify drivers of an impending } \\
\text { intersection violation }\end{array}$ \\
\hline Curve Speed Warning (CSW) & $\begin{array}{l}\text { Notify drivers of an upcoming sharp turn } \\
\text { when vehicle speed is too fast }\end{array}$ \\
\hline Lane Departure Warning (LDW) & $\begin{array}{l}\text { Notify drivers when the vehicle drifts } \\
\text { outside of the lane markings }\end{array}$ \\
\hline Lane Change Warning (LCW) & $\begin{array}{l}\text { Notify drivers making a lane change of an } \\
\text { impending collision with a vehicle in the } \\
\text { adjacent lane }\end{array}$ \\
\hline Park Assist & $\begin{array}{l}\text { Provide feedback to drivers parking a } \\
\text { vehicle regarding the distance to } \\
\text { surrounding objects }\end{array}$ \\
\hline Adaptive Cruise Control & $\begin{array}{l}\text { Automatically adjusts the vehicle's cruise } \\
\text { speed based on lead vehicle speed }\end{array}$ \\
\hline Night Vision & $\begin{array}{l}\text { Uses infra-red light to display non- } \\
\text { illuminated objects to drivers at night }\end{array}$ \\
\hline All Around Camera View & $\begin{array}{l}\text { Displays video images of areas } \\
\text { surrounding the vehicle }\end{array}$ \\
\hline Speed Limit Assistant & $\begin{array}{l}\text { Captures speed limit with machine vision } \\
\text { and displays it on the speedometer }\end{array}$ \\
\hline Alcohol/Drowsy Detection & Alerts driver when drowsiness is detected \\
\hline Emergency Braking & $\begin{array}{l}\text { Automatic engagement of vehicle brakes } \\
\text { in response to a forward threat }\end{array}$ \\
\hline Electronic Stability Control & $\begin{array}{l}\text { Detects over- and under-steer and applies } \\
\text { power and brakes to individual wheels to } \\
\text { correct for it }\end{array}$ \\
\hline
\end{tabular}

\section{WHY ALERT DRIVERS?}

In general, people are safe drivers. They develop spatial awareness of surrounding objects and the pathways available to safely navigate around them by visually sampling the forward roadway, the rear view mirror, their side view mirrors, and their blind spots. Mental resources are used to store this information in working memory where it is analyzed and acted upon through selection of a response, such as applying the vehicle's brakes, or steering into an adjacent lane. Drivers, however, are limited in that they can only visually attend to one location at a time. Inattention as well as rapidly changing traffic conditions can undermine drivers from developing accurate spatial awareness. Safety is jeopardized when drivers execute a maneuver based on inaccurate mental models of their environment. The 100-Car naturalistic driving study found that nearly 80 percent of all crashes and 65 percent of all near-crashes involved driver inattention (due to distraction, fatigue, or just looking away) just prior to (i.e., within 3 seconds) the onset 
of a conflict (Dingus, et al., 2006). Additionally, Fitch et al. (2009) found that drivers involved in lane change crashes and near-crashes failed to adequately check their sideview mirrors or blind spots prior to changing lanes. These findings suggest that drivers missed a critical aspect of their environment because they assumed circumstances would not change as they looked away. As an analogy to this problem, consider the issue of sampling frequency in analog-to-digital conversion, where transients in an analog signal can be missed if the sampling frequency is inadequate.

Active safety systems stand to improve transportation safety by mitigating driver inattention and poor visibility. By providing information on the location of surrounding traffic, these systems support the perception of changing conditions that might otherwise go unnoticed by drivers looking down at their cell phone, driving in inclement weather, facing increased time pressure to change lanes, or who lack the required range of motion to check their blind spots. Additionally, since driver visual search strategies are a direct reflection of their expectancies (Wickens \& Hollands, 1999), active safety systems support drivers in detecting objects located in areas they were not expected to be. This information can be supplemental to the driver's existing sampling strategy, which may possibly increase crash threat detection reliability. Active safety systems may also reduce mental workload and alleviate stress, which may normalize aggressive driving behavior (SAE/Automotive Engineering International, 2007). For rapidly evolving crash threats, active safety systems can alert the driver to make an immediate collision avoidance maneuver. It is expected that with these systems, drivers will be able to respond to the collision earlier, reducing their impact velocity and collision severity, or allow them to avoid the collision altogether. For instance, Fitch, Rakha, et al. (2008) estimated that a nation-wide deployment of an FCW system in heavy vehicles could reduce the number of RE crashes by 21 percent.

It is foreseeable that technology may allow the driving task to someday become completely automated. Assuming system reliability and trustworthiness are adequately addressed when this occurs, it may be inappropriate to warn drivers of an impending collision. This is because drivers that are aware they are about to collide with an object tend to brace themselves before impact. This tensing up of the muscles can propagate bodily injury by increasing the forces transferred to the body upon impact (Tencer, et al., 2002). In the mean time, CAS designers must concern themselves with what functions are to be performed by the driver and which ones are to be performed by the vehicle.

Automated braking in stop-and-go traffic has become a commercially available feature on certain Mercedes-Benz as well as Volvo vehicles. However, machines are not yet capable of reliably differentiating true crash threats from benign objects. The decision of whether the driver or vehicle should brake for all circumstances is therefore a concern. Hard braking to false-alarms can have unintended consequences given the expectancy of following-vehicle drivers. On the other hand, legal issues may ensue if evasive action is not properly executed by a vehicle. Although humans are better at discerning true crash threats from false alarms, machines are better at vigilantly monitoring the forward roadway. Driver inattention from secondary tasks, as well as fatigue, can significantly delay response time to crash threats. Furthermore, some drivers are physically incapable 
of engaging a vehicle's maximum braking in emergency situations (Fitch, et al., In Press). It may therefore be appropriate for vehicles to automatically brake at the last possible second before a collision occurs if the driver has not responded. Integrating information regarding the driver's gaze may improve the reasoning behind such algorithms.

It should be noted that function allocation is an area of inquiry in Human Factors that provides methods to support system designers in determining which functions should be performed by machines and which ones should be performed by humans (Chapanis, 1996). However, as machines become increasingly reliable at performing life-critical functions, the decision of whether to not allocate these functions to them has become increasingly difficult. New function allocation methods are needed to address this dilemma (Kleiner, 1998).

\section{CRASH ALERT STRATEGIES}

How active safety systems support drivers in perceiving changing traffic conditions and alert drivers to crash threats is critical to their effectiveness. False alarms and nuisance alarms are particularly concerning in this matter. False alarms are alerts that are generated when the active safety system does not function as designed (e.g., when an electronic sensor interprets ambient noise as a signal) (Barfield \& Dingus, 1998). Nuisance alarms, on the other hand, are alerts that are generated by a properly functioning active safety system, but are uninformative to the driver because the situation does not constitute a crash threat. Frequent false alarms and nuisance alarms have been shown to have detrimental effects on the user's trust in the system. The first generation Traffic-Collision Alerting System for commercial aircraft produced so many false and nuisance alarms in congested traffic conditions that pilots stopped believing that the alerts were valid and ignored them (Barfield \& Dingus, 1998).

Methods of overcoming the paradox of providing reasonably conservative warnings while minimizing false alarms have been proposed. One option is the use of graded alerts (Barfield \& Dingus, 1998). Graded alerts present drivers with a sequence of cautionary to imminent warnings as a crash threat develops. Graded alerts have been recommended instead of single-stage crash alerts, which are presented immediately before a collision (Dingus, et al., 1997; Lee, Hoffman, \& Hayes, 2004). This is because graded alerts allow drivers to directly perceive the relationship between other vehicles and their own (e.g., the headway and the relative velocity between the vehicles). Drivers can use this information to make timely changes in their driving behavior. Graded alerts have been found to lead to the development of greater safety margins (Dingus, et al., 1997; NHTSA, 2005; Volvo, 2005) and a lower rate of inappropriate responses to nuisance alerts (Lee, et al., 2004). With graded alerts comes another recommendation that there be a parallel change in alert modality as the alerts' grade changes from cautionary to imminent (Barfield \& Dingus, 1998). For example, visual alerts could be used to present cautionary warnings to drivers, while auditory alerts could be used to present imminent warnings. Changing the alert modality helps notify the driver that an immediate response is required. 
It may also be possible to reduce nuisance alarms using attention-centric systems. Attention-centric systems are notification systems that adapt how they present information to users (McCrickard \& Chewar, 2005). An attention-centric active safety system may adapt to drivers by tracking their eyeglances and only generate crash alerts when the driver is not looking in the direction of the crash threat. Attention-centric systems is a research area in Human-Computer Interaction where developments stand to benefit active safety systems.

\section{CRASH ALERT MODALITY}

The modality used to present crash alerts is also an important human factors consideration. This section discusses the presentation of crash alerts through visual, auditory, and haptic modalities.

Visual alerts warn drivers of approaching danger by presenting an icon or by flashing a light on a display (General Motors Corporation, 2005). An example of a visual alert would be the low-fuel icon that illuminates on the vehicle's dashboard when the fuel tank is nearly empty. Presenting crash alerts visually has been a controversial topic within the human factors community. Visual alerts are capable of presenting highly informative warnings, they are reviewable after they have been evoked, and they are perceived to be less annoying than non-visual alerts. Visual alerts are also effective at clarifying crash alerts to drivers. On the other hand, visual alerts can undermine crash alert effectiveness by demanding the driver's visual attention when it should be focused on the actual hazard. Given these considerations, visual alerts may be more appropriate for the presentation of cautionary early stage alerts in a graded alert approach.

Non-visual alerts, such as auditory and haptic alerts, are good for presenting imminent final stage alerts in a graded alert approach. This is because they are perceptible regardless of where the driver is looking. Presenting complex information through nonvisual alerts is challenging. When a vehicle is outfitted with a single CAS, such as an FCW system, drivers can learn that a non-visual alert is intended to cue their attention to the forward roadway. However, for vehicles outfitted with multiple CASs, the alerts must be differentiable to be comprehended. Otherwise, drivers may waste valuable time looking at a visual display for clarification of the threat type. Non-visual alerts that provide directional information may help resolve this issue. Both auditory and haptic alerts have been considered for the final stage of a graded alert approach. The appropriateness of each alert modality for this application is reviewed below.

Auditory alerts warn drivers of approaching danger by generating sounds. An example of an auditory alert would be a beep that is heard when one's safety belt is not fastened. Auditory alerts possess an innate attention grabbing quality. They are also good at conveying urgency to the driver (Edworthy, 1998). Properly designed, they can reduce response time to crash threats. McElheny (2005) found that an auditory CSW alert significantly reduced throttle and brake response times, as well as curve entry speed for drivers approaching a curve at $89 \mathrm{~km} / \mathrm{h}(55 \mathrm{mph})$. On the other hand, auditory alerts must be presented loud enough that they are detectable in the presence of noise. Design 
guidelines recommend that auditory alerts be 15 decibels $^{1}(\mathrm{~dB})$ louder than the noise sound pressure level (Karwowski \& Marras, 1999). Auditory alerts evoked at $75 \mathrm{dBA}^{2}$ have been shown to be audible inside a vehicle (Fitch, Kiefer, Hankey, \& Kleiner, 2007). However, they can annoy drivers when they are not merited (Fitch, Kleiner, Kiefer, Lee, \& Babski-Reeves, 2005).

Spatial audio has been proposed for communicating crash threat direction to drivers (Fitch, Kiefer, Hankey, et al., 2007). The concept involves emitting a sound from the direction the threat is detected. Provided drivers successfully localize the alert, their attention can be focused towards a specific direction, allowing them to address the crash threat. Drivers localize the alerts using perceived differences in sound wave timing and intensity between each ear. These cues are minute and depend on the auditory alert frequency. For instance, people are better at localizing sounds below $1500 \mathrm{~Hz}$ with timing cues and sounds above $3000 \mathrm{~Hz}$ with intensity cues (Sanders \& McCormick, 1993). Sound localization inside a vehicle is challenging with the acoustical complexity of the cabin interior. Sounds bounce off the windshield, roof, seats, and floor, making it difficult for drivers to perceive the true origin of the sound. Fitch, Kiefer, Hankey, et al. (2007) investigated drivers' ability to localize auditory alerts using a spatial audio display that comprised the vehicle's stock sound system. The display used a higher pitched broadband alert with embedded sound impulses to indicate the front-left, front, and frontright direction, while a lower pitched broadband alert with embedded sound impulses to indicate the left, right, back-left, back-right, and back directions. Overall, drivers localized the auditory alerts with $32 \%$ accuracy, a reflection of the task's difficulty. Tan and Lerner (1996) investigated the use of spatial audio inside a vehicle for conveying directional alerts. They used various combinations of sixteen speakers mounted around the interior of the vehicle cabin to generate the auditory alerts. They found that auditory alerts were not appropriate for precise localization. Since the spatial audio alerts are localized using perceived differences between the drivers' ears, spatial audio displays require drivers to have two properly functioning ears. Hearing decrements from aging, hearing damage from trauma, as well as ambient noise from open windows, HVAC (Heating Ventilation Air conditioning) system, or passenger conversation affect localization performance. Despite these drawbacks, auditory alerts should not be discounted as an alert component. For instance, they are good for presenting nondirectional alerts. Additional research may prove them to be effective in specific circumstances.

Haptic alerts warn drivers of approaching danger by generating vibrations or by applying forces to the driver. An example of a haptic alert would be a cell phone that vibrates during an incoming call. Haptic alerts may be more appropriate for presenting collision warning alerts. Fitch, Kiefer, Hankey, et al. (2007) evaluated a directional haptic seat's ability to communicate a direction to drivers while they drove down a four-lane highway at $89 \mathrm{~km} / \mathrm{h}(55 \mathrm{mph})$. The haptic seat conveyed eight directions by vibrating eight areas in the seat pan (bottom of driver seat). They found that drivers correctly localized $86 \%$

\footnotetext{
${ }^{1}$ Decibel is the standard measure of sound pressure level, or intensity.

${ }^{2}$ Since a given sound is perceived to have different intensity when its frequency is changed, a dBA weighting system is used to scale the intensity of sounds relative to what the human ear would perceive.
} 
of the alerts. They also found that $90 \%$ of the localization errors made resulted from drivers mistaking the alert for a location spatially contiguous (one location off) to the actual location. Van Erp and Van Veen (2004) investigated the use of a haptic seat to display navigation cues to drivers. The haptic seat communicated a left or right direction by vibrating tactors under the left or right leg, respectively. By using this under-loaded modality, driver mental workload was reduced, allowing them to respond to the directional cues faster than when they were presented through a visual display.

Reasons for using haptic seat displays to communicate directions are as follows. For one, the drivers' frame of reference remains relatively stable (Fitch, Kiefer, Hankey, et al., 2007). Information can be transmitted if the driver takes their foot off the accelerator, holds the steering wheel with one finger, or even their knees. The haptic alerts are also personal. They do not expose the driver to passenger criticism or disturb in-vehicle activities such as audio/visual entertainment or a sleeping child (Fitch, Kiefer, Hankey, et al., 2007). Thirdly, compared to auditory alerts, haptic seat alerts have been reported to be less annoying (Fitch, et al., 2005), which may be more appropriate given the existence of nuisance alarms. Additionally, although haptic sensitivity decreases with age (Verrillo, Bolanowski, \& Gescheider, 2002), haptic seat alerts may be more reliably perceived than auditory alerts by elderly drivers owing to the prevalence of hearing loss in one or both ears (Fitch, Kiefer, Hankey, et al., 2007). For these reasons, directional haptic seats appear to be a promising method of alerting drivers to a crash threat.

\section{RESEARCH OBJECTIVES}

\section{Research Goals}

This dissertation was written with the goal of furthering the scientific knowledge of drivers' ability to process integrated CAS alerts presented through a haptic driver seat. Specifically, this research set out to quantify the effects of incorporating various alerts into an integrated CAS architecture. There was also a desire to understand how driver response performance differed when the haptic alerts were presented through common versus unique areas in the driver seat. The effectiveness of the haptic driver seat's ability to direct distracted drivers' attention to an actual forward crash threat was also a focus. These goals were pursued by conducting Human Factors experiments designed to answer the following research questions.

\section{Research Questions}

\section{Research Question 1: What is the effect of increasing the number of CAS alerts on driver performance?}

Technological advancements have made it possible for vehicles to monitor a multitude of safety dimensions and warn drivers when any single safety dimension is breached (www.its.dot.gov, 2006). A direction active safety systems are taking is for each system to provide context through the presentation of a unique alert rather than using a generic master alert for every system. An interest exists in developing a haptic driver seat that effectively communicates multiple alerts, such as FCW, CSW, IVW, LCW, and LDW 
alerts, to drivers. However, designers should question whether drivers can quickly and accurately comprehend this much information when these CASs are integrated.

Information Theory, which arose from Shannon and Weaver's work at Bell Telephone Labs in the United States to develop a mathematical theory of communication (1949), states that the amount of information in a stimulus is a function of: a) the number of differing events presented, b) the probability that an event will occur, and c) the sequential constraints imposed on the occurrence of an event (Wickens \& Hollands, 1999). Hick (1952) and Hyman (1953) applied Information Theory to explain human performance in a choice response task. In doing so, they established the Hick-Hyman Law, which states that the more information a stimulus possesses, the longer humans take to process it. This suggests that drivers' response time to any CAS alert may increase as the number of different CAS alerts increases. Here, the additional attentional resources required to process which alert was presented and which response is appropriate translate into increases in response time.

Three years later, George Miller (1956) applied Information Theory to explain human performance in an immediate memory task. He found that people's ability to remember a string of digits decreased as the number of digits increased. His research showed that people, in general, can effectively process 2 to 3 bits of information before performance significantly degrades. Miller concluded that people's span of immediate memory is approximately $7 \pm 2$ chunks of information. The implication of this research is that drivers should be able to comprehend $7 \pm 2$ different types of haptic seat alerts.

The first research question attempts to quantify the effects of increasing the amount of information conveyed by haptic driver seat alerts on drivers' response performance (i.e., how fast and accurate drivers are in performing correct driving maneuvers in response to CAS alerts). The research previously described suggests that drivers' response performance will degrade as the number of alerts increases.

\section{Research Question 2: What is the effect of the haptic seat alert location on drivers' ability to differentiate meaningful from uninformative alerts?}

Research question 2 considers scenarios drivers may encounter with an integrated CAS and how the CAS architecture might affect drivers' response performance. LDW alerts warn drivers when their vehicle drifts out of lane. These alerts stand to provide feedback to drivers that fail to track the position of their vehicle in the lane due to drowsiness, distraction, impairment, etc... LCW alerts, on the other hand, warn drivers when they are about to collide with an adjacent vehicle when executing a lane change. LCW alerts stand to benefit drivers who fail to observe an adjacent vehicle when changing lanes. An analysis of driver behavior leading to the lane change crashes and near-crashes observed in the 100-Car Study found that less than half of the involved drivers looked at their sideview mirrors, side windows, and rear-view mirrors during the last $3 \mathrm{~s}$ prior to performing lane changes (Fitch, et al., 2009). It has been proposed that LCW systems remain enabled even if drivers do not use their turn signals (Campbell, Richard, Brown, \& McCallum, 2007). This is merited because Fitch et al. (2009) found that 85 percent of drivers involved in lane change crashes and near-crashes used their turn signal during 
planned left lane changes, while only 24 percent used their turn signal when swerving into an adjacent left lane to avoid a forward crash threat. LCW systems that only enable with turn signal activation would thus miss a portion of lane change events.

Given LCW systems would always be enabled, consider the case of drivers who fail to activate their turn signal when changing lanes. Such drivers would receive LDW alerts as they cross over into the adjacent lane. With repeated exposure to these benign alerts, these drivers may adapt and ignore them. At the same time, these drivers may fail to perceive an adjacent vehicle when changing lanes. Here, these drivers would receive LCW alerts as they execute the lane change. Since both LDW and LCW alerts warn drivers of lateral crash threats, it is foreseeable that haptic seat designers elect to present both alert types through the same seat location to reduce manufacturing costs. However, haptic seat designers should question whether drivers can quickly differentiate meaningful LCW alerts from potentially uninformative LDW alerts. Quick response times to lane change crash scenarios are imperative. Fitch et al. (2009) found that nearcrashes arising from a vehicle executing a planned left lane-change across the path of an adjacent vehicle occurred on average in $2.3 \mathrm{~s}$. The second research question asks whether generating LDW and LCW alerts through different areas in the driver seat improves driver response performance compared to when both alerts are generated through the same area in the seat. Drivers are expected to improve their performance when the alerts are presented through different locations in the driver seat compared to when they are presented through the same location. The results of this study are expected to assist designers in determining the location of the haptic seat alerts.

\section{Research Question 3: What is the effect of a haptic seat alert on drivers' collision avoidance behavior?}

The effectiveness of a haptic driver seat to alert distracted drivers to an actual crash threat is relatively unknown. Research to date has asked participants to either imagine crash contexts (Fitch, Kiefer, Hankey, et al., 2007), or exposed them to simulated crash threats (Ho, Reed, \& Spence, 2006; Lee, et al., 2004), when experiencing haptic alerts. The third research question investigates drivers' crash avoidance performance to a surprise crash threat when receiving a haptic seat alert. Drivers' collision avoidance behavior is expected to improve upon receiving haptic seat alerts. 


\section{CHAPTER 2. LITERATURE REVIEW}

This chapter reviews the relevant literature on the design of haptic seat displays. The physiology of haptic perception is explained first. Factors that are known to affect perception of vibration stimuli are then reviewed. The chapter concludes with the presentation of vibrotactile display design considerations.

\section{HAPTICS DEFINED}

The word "Haptic" comes from the Greek word haptesthai, which means "relating to or based on the sense of touch" (Webster's Ninth New Collegiate Dictionary, 1985). In the Human Factors literature, however, it is used interchangeably with the four somatic sensory system modalities: 1) tactile (touch), the sensation elicited by mechanical stimulation of the skin, 2) proprioception, the sensation elicited by the mechanical displacement of the muscles and joints, 3) pain, which is elicited by stimuli of sufficient intensity to damage tissue, and 4) thermal sensation, which is elicited by cool and warm stimuli (Boff \& Lincoln, 1988).

\section{PHYSIOLOGY OF HAPTIC PERCEPTION}

The skin functions to protect the human body from injury, desiccation, and infection, as well as regulate body temperature (Merchant, 2007). Ranging from 1.2 to $2.3 \mathrm{~m}^{2}$ in surface area, it is the human body's largest organ. Comprising $16 \%$ of the total body weight, it is also the heaviest organ (Merchant, 2007). Skin contains a variety of receptors that allow the human body to sense objects contacting or proximally located to it (Mortimer, Zets, \& Cholewiak, In Press). There are four major functional groupings for these receptors: 1) mechanoreceptors, 2) proprioceptors, 3) thermoreceptors, and 4) nocioreceptors (Verry, 1998). Mechanoreceptors are sensitive to pressure, vibration and slip, proprioceptors are sensitive to the position of the human body, thermoreceptors are sensitive to changes in temperature, and nocioreceptors are responsible for sensing pain. Since this dissertation focuses on the communication of information through vibration, this review is limited to the physiology of mechanoreceptors.

\section{Factors Affecting Perception of Vibration}

The perception of vibration is determined by the type of mechanoreceptor, the number, distribution, and placement of the mechanoreceptors, the structure and location of the skin, as well as the presence of a masking stimulus. This section reviews these four elements.

\section{Types of Mechanoreceptors}

There are four types of mechanoreceptors: Pacinian corpuscles, Meissner's corpuscles, Merkel's disks, and Rufinni cylinders (Cholewiak \& Ciollins, 1991; Greenspan \& Boloanowski, 1996). Figure 1 depicts these mechanoreceptors through a histological diagram (Greene, 2007). Each mechanoreceptor differs in terms of how it sends signals to the central nervous system (CNS) and its sensitivity to stimulation. Because 
mechanoreceptors send signals to the CNS they are classified as afferents. The four mechanoreceptors classify into two afferent types: fast adapting and slow adapting. Fast adapting (FA) (and also referred to as rapid adapting and quick adapting in the literature) afferents only briefly send signals to the brain upon stimulation. Here, the signal quickly decays after stimulus onset. FA mechanoreceptors are the reason why people stop feeling their clothes once they are worn. Slow adapting (SA) afferents, however, continue to send signals to the brain throughout sustained indentation of the skin.

A mechanoreceptors sensitivity profile, termed its receptive field, describes the range of sensitivity to mechanical deflection. Two-dimensional sensitivity profiles have been obtained for individual mechanoreceptors by moving a point probe over the skin surface and recording the receptor's response. Sensitivity is greatest directly above the receptor, and decreases as the distance from the center of the field increases (Macefield, 1998). The receptive field is typically circular or oval in shape. The four mechanoreceptors classify into two receptive field sizes: Type I and type II. Type I afferents have small, well-defined receptive fields, whereas type II afferents have large receptive fields whose borders are difficult to determine. Further detail on the four mechanoreceptors is provided next. 


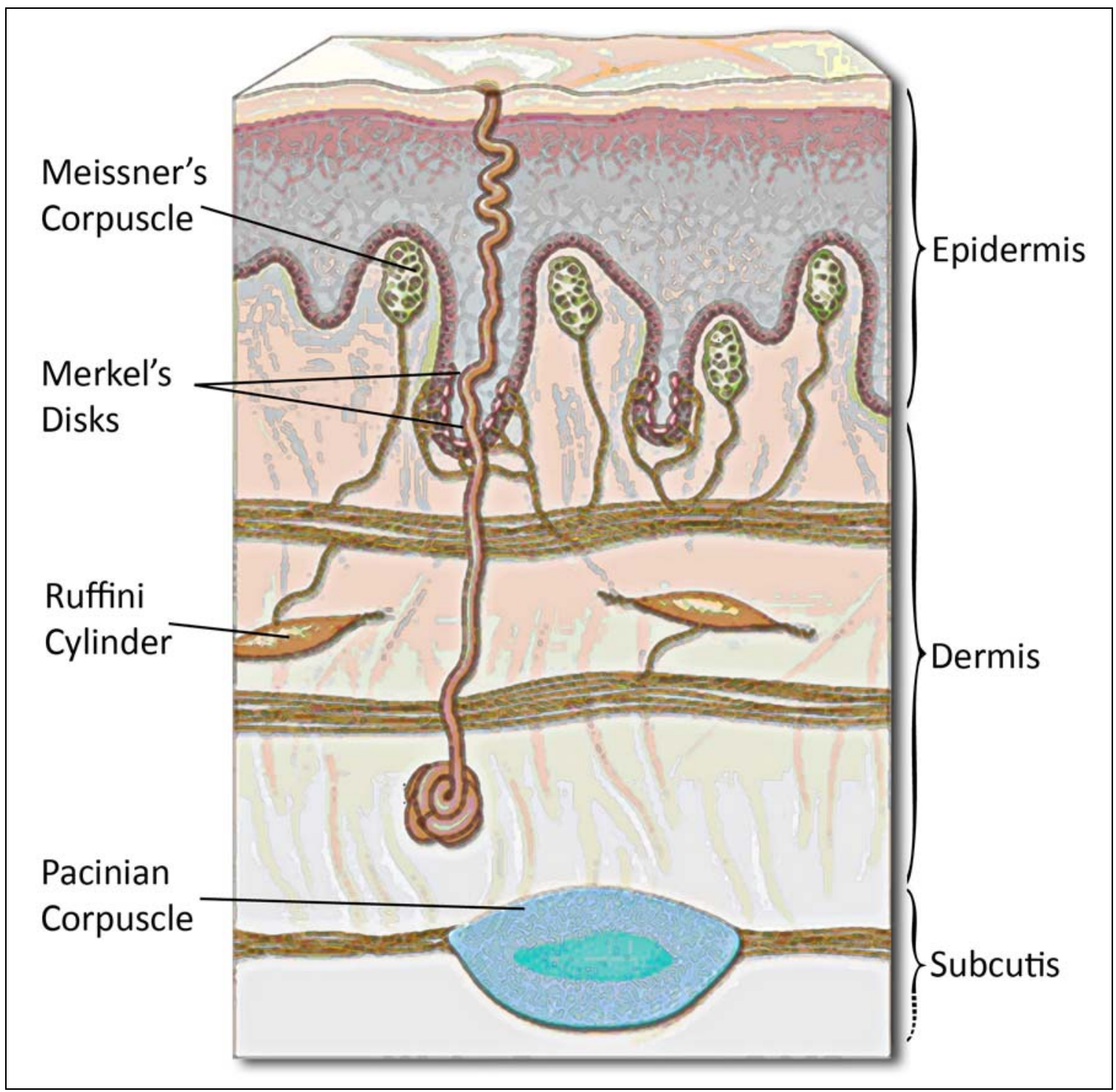

Figure 1. Histological depiction of four mechanoreceptors in glabrous skin. Adapted from Greene (2007).

\section{Pacinian Corpuscles}

Pacinian corpuscles are FA II afferents that detect momentary stimulus changes (Macefield, 1998). They are responsible for detecting gross pressure changes and high frequency vibrations. The exact range of their sensitivity varies by study. Greenspan \& Boloanowski (1996) report it as being between $40-500 \mathrm{~Hz}$, while personal communication with Lederman, an expert in the field, reports it as being between 50 and $700 \mathrm{~Hz}$ (Lederman, 2008). Gescheider \& O'Malley (1983) report that Pacinian Corpuscles are most sensitive to vibrations between 200 and $400 \mathrm{~Hz}$, while Lederman reports it as being between 200 and $300 \mathrm{~Hz}$. Located in the subcutis, Pacinian corpuscles are the largest of the skin receptors measuring approximately $1 \mathrm{~mm}$ in length. 


\section{Meissner's Corpuscles}

Meissner's corpuscles are FA I afferents (Macefield, 1998). They are distributed throughout the skin, but are concentrated in highly sensitive areas such as the fingertips and lips. Having a smaller receptive field, they are responsible for detecting low frequency vibrations and generating the sensation of flutter (which is the sensation generated when the skin is stimulated with a point probe vibrating close to $40 \mathrm{~Hz}$ ). Greenspan \& Boloanowski (1996) report that they are sensitive to frequencies between 2 and $40 \mathrm{~Hz}$ and have peak sensitivity at $40 \mathrm{~Hz}$, while Lederman reports that their sensitivity ranges between 5 and $50 \mathrm{~Hz}$ (Lederman, 2008). They are located in the dermis above the subcutis and are between $30-140 \mu \mathrm{m}$ in length and $40-60 \mu \mathrm{m}$ in diameter.

\section{Merkel’s Disks}

Merkel's disks are associated with SA I afferents (Macefield, 1998). They are responsible for the perception of texture and shape. They produce a sensation of "pressure" for stimuli between 0.4 and $2 \mathrm{~Hz}$ (Greenspan \& Boloanowski, 1996). Lederman (2008) reports that they are sensitive to frequencies between 0.5 and $5 \mathrm{~Hz}$. Pacinian and Meissner's corpuscles will notify the human body when it is initially touched, while Merkel's disks continue to notify the body that the object is still in contact. Merkel's disks are extremely sensitive and may respond to skin deflections less than $1 \mu \mathrm{m}$. Merkel's disks are located in the upper layers of the skin (dermis).

\section{Rufinni Cylinders}

Rufinni cylinders are associated with SA II afferents (Macefield, 1998). They respond best to lateral skin stretch. They also produce a buzz-like sensation in response to frequencies between $100-500 \mathrm{~Hz}$ (Greenspan \& Boloanowski, 1996). In non-glabrous (hairy) skin, Rufinni cylinders have a higher representation relative to the other mechanoreceptors.

\section{Number, Distribution, and Placement of Mechanoreceptors}

The number, distribution, and placement of mechanoreceptors affect the perception of vibration (Lederman \& Browse, 1988). This is simply an issue of bandwidth, where an increased number of receptors facilitate improved perception of tactile information. The density of mechanoreceptors varies throughout the body. It has been estimated that of the 2000 Pacinian corpuscles in the human body, approximately a third of them are located in the digits (Vallbo \& Johansson, 1978). It has also been estimated that there are 17,000 mechanoreceptors in the human hand (Lederman \& Browse, 1988).

\section{Skin Structure and Location}

The type of skin affects the human body's sensitivity to vibration. For example, in glabrous (smooth) skin, Pacinian corpuscles are the most sensitive of the four mechanoreceptors and can detect displacements less than $1 \mu \mathrm{m}$ at $250 \mathrm{~Hz}$. In hairy skin, however, overall sensitivity is much lower (Mortimer, et al., In Press). Although precise 
measurement is difficult since Pacinian-like responses are obtained from surrounding hair follicles, the sensitivity of the Pancinian mechanoreceptor is estimated to be $1 / 10$ the sensitivity of it in glabrous skin (Bolanowski, Gescheider, \& Verrillo, 1994). However, detection of high frequency stimuli is not limited to Pacinian corpuscles. The range of frequencies detected by slow adapting afferents extends from 20 to $100 \mathrm{~Hz}$ in nonglabrous skin.

Sensitivity is also dependent on the location of the stimulus on the human body. Twopoint resolution (the ability to discriminate two-point from one-point touch) for pressure can be as small as a few millimeters on the fingertip, and as large as $50 \mathrm{~mm}$ on the calf (Figure 2) (Greene, 2007). Vibration sensitivity threshold also varies by body location. Sensitivity is as high as a few deci-microns on the hand, and worst on the gluteus maximus (Figure 3) (Boff \& Lincoln, 1988). 


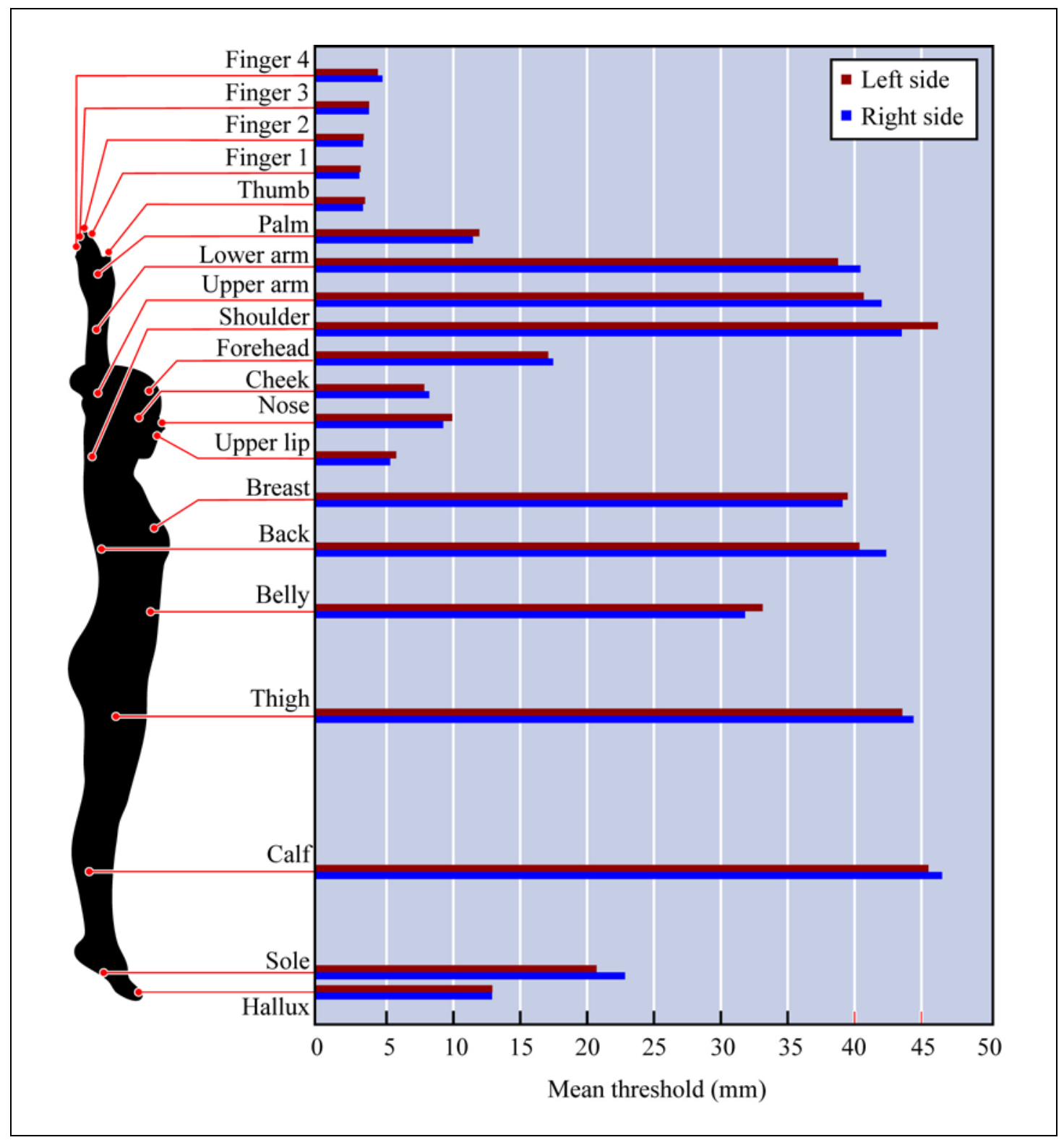

Figure 2. Two-point discrimination thresholds for the human body. Adapted from Greene (2007). 


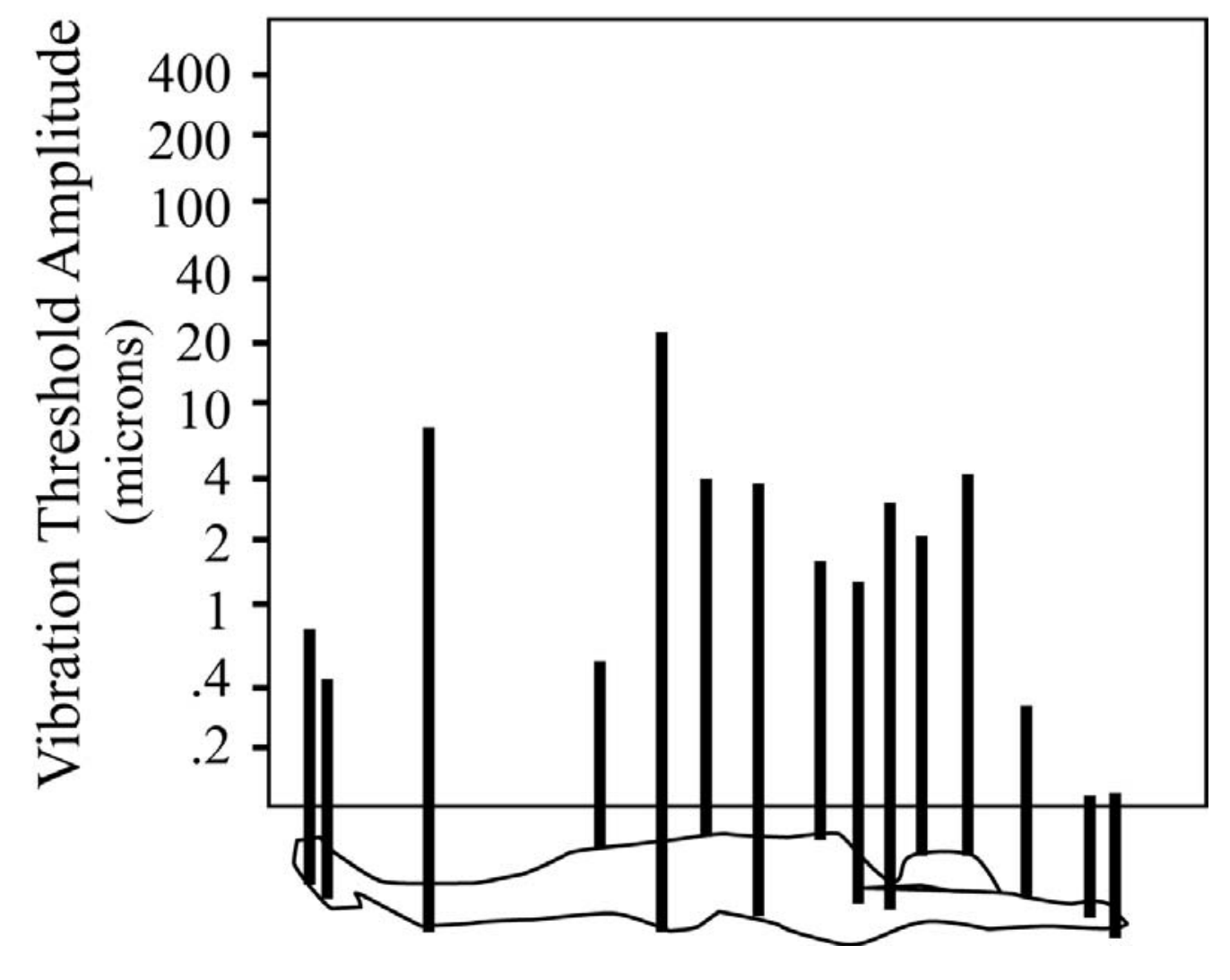

Figure 3. Thresholds for $200 \mathrm{~Hz}$ vibration for one male subject. Adapted from Boff and Lincoln (1988).

\section{Masking Effects}

Masking refers to the reduction in detectability of a tactile stimulus due to the presence of another tactile stimulus that is overlapping in time and/or space (Boff, Kaufman, \& Thomas, 1986). There are three kinds of spatial temporal masking: 1) lateral masking, 2) temporal masking, and 3) metacontrast masking.

\section{Lateral Masking}

Lateral masking occurs when one haptic stimulus overlaps another in time, but not in space (although the two stimuli must be spatially contiguous) (Boff, et al., 1986). Lateral masking is exemplified by Loomis (1983) who compared the tactile legibility of two Braille character sets. Set 1 was the Standard English Braille alphabet, while set 2 placed a rectangular border around each letter in set 1 (Figure 4). Tactile recognition accuracy for set 1 was over $80 \%$ correct, while recognition accuracy for set 2 was under $10 \%$ correct. The addition of the same surround to each Braille character greatly reduced its detectability. 


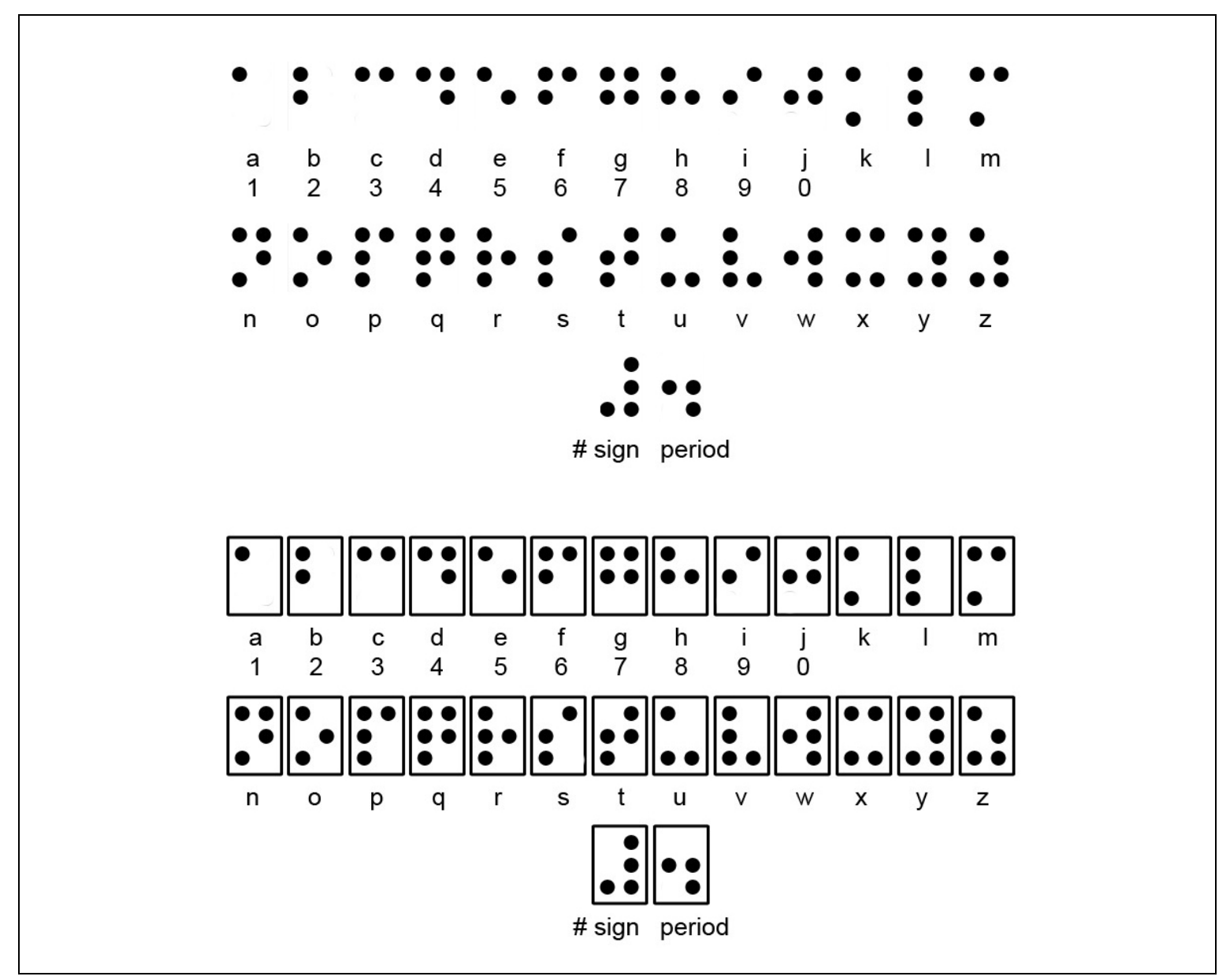

Figure 4. Upper: Standard English Braille alphabet. Lower: Standard English Braille alphabet with border. Adapted from Loomis (1983).

\section{Temporal Masking}

Temporal masking occurs when two haptic stimuli occupy the same location at different points in time (Boff, et al., 1986). The degree to which the two stimuli interfere with each other is dependent on their format. For example, a masking stimulus consisting of a temporally varying pattern increases tactile sensation threshold more than a masking stimulus consisting of continuous activation (Boff, et al., 1986). This is true even if the temporally varying pattern has less energy (number of activation units) than the continuous activation stimulus. Interference also increases when the masking stimulus duration increases (Boff, et al., 1986). Temporal masking is affected by the sequential order of the stimuli. Backward masking, which occurs when the masking stimulus is presented after the signal stimulus, generates more interference than forward masking, which occurs when the masking stimulus is presented before the signal stimulus (Boff, et al., 1986). 


\section{Metacontrast Masking}

Metacontrast masking occurs when the signal stimulus and masking stimulus overlap neither in time or in space (although they are close together in space and time) (Boff, et al., 1986). Maximum metacontrast masking occurs when the masking stimulus follows the signal stimulus close to $50 \mathrm{~ms}$, and not when they are presented simultaneously (Boff, et al., 1986).

\section{Perceived Intensity of Vibratory Stimuli}

The decibel $(\mathrm{dB})$ is the most common measure for vibrotactile stimuli intensity. It is defined as

$$
d B=20 \log \left(\frac{D_{1}}{D_{\text {ref }}}\right)
$$

(Greenspan \& Boloanowski, 1996)

where,

$\mathrm{D}_{1}$ is the skin displacement produced by the stimulus

$D_{\text {ref }}$ is a reference value, usually $1.0 \mu \mathrm{m}$ peak

The perceived magnitude of tactile sensations is related in an orderly fashion to the intensity of the haptic stimulus (Greenspan \& Boloanowski, 1996). Weber's law states that there is a linear relationship between the intensity of the stimulus and the incremental increase of a stimulus from that level to obtain a just noticeable difference (JND) (Weber, 1978). In essence, the increment in stimulus intensity required for someone to perceive a difference in intensity is a constant proportion of the original intensity (Greenspan \& Boloanowski, 1996). Recent studies assessing the JND to vibratory stimuli have found values ranging from 0.05 for an amplitude of $0.4 \mathrm{~dB}$ to 0.3 for amplitudes of $2.3 \mathrm{~dB}$ (Greenspan \& Boloanowski, 1996).

\section{Contours of Haptic Sensation}

Although Greenspan and Boloanowski (1996) cite the frequency response for the four mechanoreceptors as ranging from $0.4 \mathrm{~Hz}$ to $500 \mathrm{~Hz}$, they state that the human body's overall sensitivity to vibrotactile stimuli extends from $20 \mathrm{~Hz}$ to $700 \mathrm{~Hz}$ (Greenspan \& Boloanowski, 1996). For frequencies between $20-40 \mathrm{~Hz}$, the perceived intensity of vibrotactile stimuli is frequency-independent (Greenspan \& Boloanowski, 1996). For frequencies between $40-700 \mathrm{~Hz}$, the perceived intensity of vibrotactile stimuli is frequency dependent (Greenspan \& Boloanowski, 1996). Maximum sensitivity occurs from stimuli vibrating close to $250 \mathrm{~Hz}$ (Greenspan \& Boloanowski, 1996). Equal subjective intensity curves for vibratory stimuli, which are analogous to the equalloudness curves for hearing, are shown in Figure 5. It can be seen that the U-shaped sensitivity curves for high frequency vibrations flatten out as intensity is increased (Greenspan \& Boloanowski, 1996). 


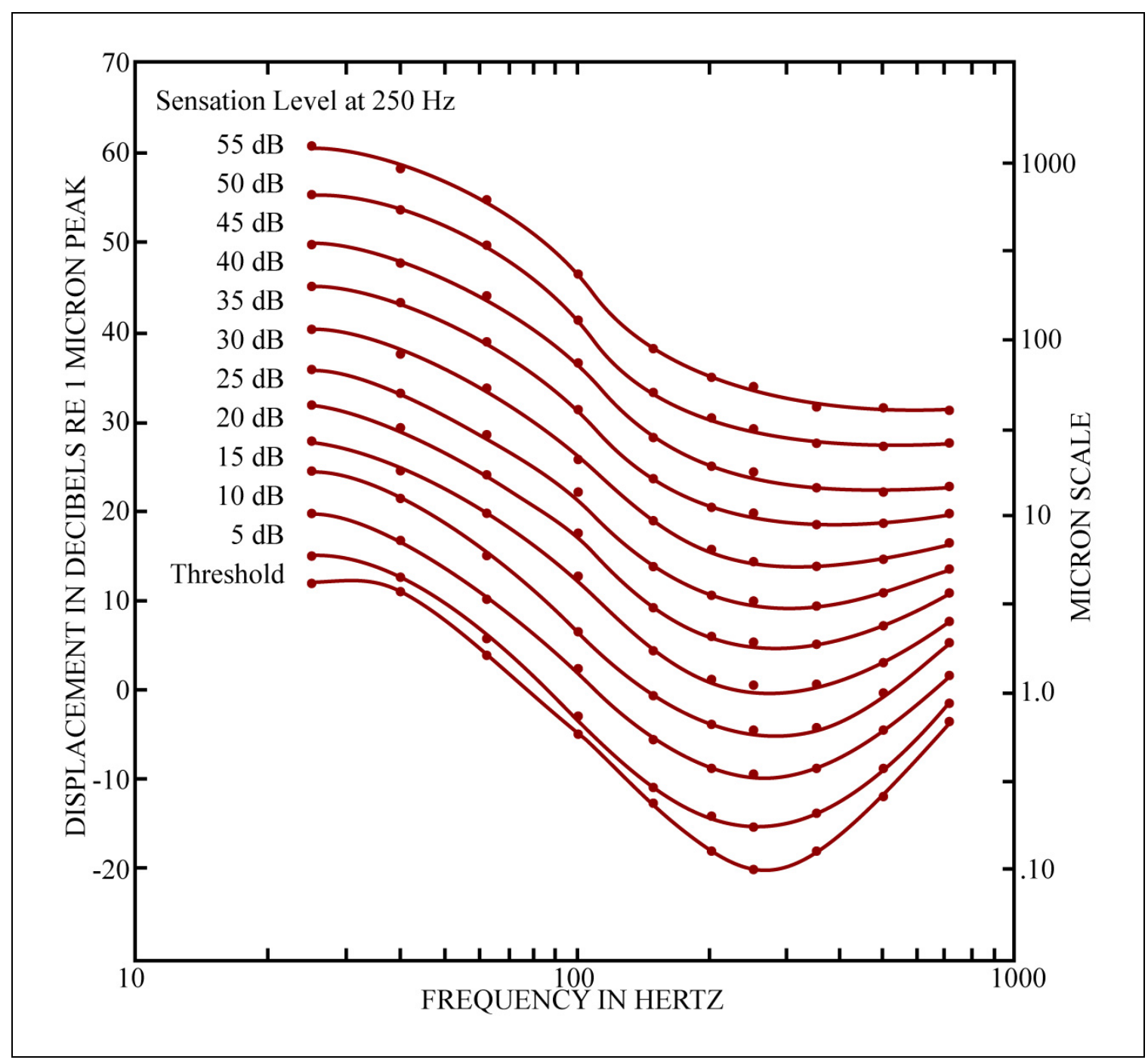

Figure 5. Equal subjective intensity curves for vibratory stimuli. Adapted from Boff and Lincoln (1988).

\section{Sensitivity Adaptation}

Adaptation refers to the reduction of sensitivity following continued stimulation. Adaptation to pressure stimuli occurs relatively quickly, which is why they are not appropriate for communicating CAS alerts. Adaptation to vibrotactile stimuli does occur, but takes over fifteen minutes to run to completion. Adaptation and recovery may take many minutes to complete (Boff \& Lincoln, 1988).

\section{Haptic Illusions}

The body's perception of haptic stimuli can be misled in the presence of certain circumstances. Three haptic phenomena that provide insight on how the body perceives haptic stimuli are presented. 


\section{Sensory Saltation}

An illusion of movement between spatial locations arises when two vibrotactile stimuli are presented at different spatial locations in rapid succession. This phenomenon is dubbed the cutaneous rabbit (Geldard \& Sherrick, 1972) owing to the feeling of progressive hops up one's arm. If the temporal separation of the stimuli is increased, the "hops" are perceived as covering a greater distance. The illusion has been observed with stimuli separated by $2 \mathrm{~cm}$ to $35 \mathrm{~cm}$. The number of pulses occurring in a location can impact the perception, with two pulses reported as too few, four to six pulses reported as better, and eighteen pulses reported as too many. For stimuli greater than $25 \mathrm{~ms}$, the inter-stimulus interval producing the greatest effect increases as stimuli duration increases (Boff \& Lincoln, 1988).

\section{Fused Sensation}

Two vibrotactile stimuli can appear as one if the inter-stimulus interval is less than $2 \mathrm{~ms}$ and the stimuli are carefully matched in perceived tactile intensity (loudness). In such cases, the perceived stimulus location may appear closest to the location of the leading stimulus. Changing the stimulus onset asynchrony from +2 to -2 ms moves the fused image from one site to the other. This illusion has been used to signal position in a prosthetic arm (Boff \& Lincoln, 1988).

\section{Shifted Location}

When presenting two vibratory stimuli in succession, the apparent location of the first stimulus can change when manipulating the inter-stimulus interval. For inter-stimulus intervals between 20 and 25-300 ms, the apparent location of the first stimulus will shift toward that of the following stimulus. The shorter the inter-stimulus interval, the greater the shift in apparent location (Boff \& Lincoln, 1988).

\section{HAPTIC DISPLAYS}

Haptic displays communicate information through contact between the device and the user's skin. Displays that transmit information to the user via vibrations are commonly called vibrotactile displays. An example of such a display is a cell phone that vibrates to notify the user of an incoming call. In contrast, displays that allow users to input commands via finger touches directly on a screen are appropriately called touch displays. An example of such displays is the Escalade kiosk manufactured by Meridian Kiosks (Meridian Kiosks, 2007) whose touch sensitive screen allows quick registration at an airport.

The research objective of this dissertation is to further understand a direction haptic driver seat's ability to communicate integrated collision avoidance alerts. An optimal haptic seat design is one that clearly communicates messages to the driver in a fashion that allows them to be quickly comprehended. Since the haptic seat alerts are intended to present critical safety messages to drivers, comprehension must be near perfect and extend across the driving population. This section presents a review of the parameters 
that have been shown to affect optimal communication of vibrotactile displays in contact with other parts of the human body. The following factors have been identified: 1) tactile activator (tactor) design, 2) tactor placement, 3) number of tactors, 4) inter-tactor distance, 5) stimulus frequency, 6) stimulus intensity 6) stimulus duration, and 7) stimulus rhythm. Design constraints are presented at the end.

\section{Tactor Design}

The device used by the vibrotactile display to stimulate the skin is called a tactor. The word is derived from "tactile activator." Tactor construction has a significant effect on how the human body perceives vibrotactile stimuli. There are three types of tactors that could be considered for use in a vibrotactile driver seat: 1) inertial shakers, 2) linear actuators, and 3 ) pneumatic tactors.

\section{Inertial Shakers}

Inertial shakers utilize the motion of an inertial mechanism to translate force to the tactor housing and consequently to the adjacent skin (Mortimer, et al., In Press). An example is the pager motor, which rotates an eccentric mass, usually a half-circular cylindrical weight, to impart momentum on the motor shaft and consequently the motor housing. The moving housing is what generates a vibration stimulus to the skin. The amount of vibration felt depends on how the motor is mounted to the housing, the mass of the motor, the mass of the eccentric rotating object, the radius from the center of the rotating object to the shaft, and the rotational velocity (Mortimer, et al., In Press). As such, the frequency (rate of rotation) and intensity are inherently linked. The frequency and intensity of the vibration is dependent on how much mass is loaded on it. Consequently, an inertial shaker placed in the driver seat pan bears the weight of the driver. As loading increases, the perceived intensity of the tactor decreases while vibration frequency increases. If the tactor bears too much weight, it may be prevented from shaking altogether (Mortimer, et al., In Press).

Inertial shakers placed in a driver seat pan, nevertheless, have been shown to effectively communicate directions to drivers (Fitch, Kiefer, Hankey, et al., 2007). This may be because the directional cues generated possessed a degree of redundancy owing to the diffuse vibrations generated by the tactors. Figure 6 shows a haptic seat display created with 64 pager motors aligned in an $8 \times 8$ array. A close-up image of one of the motors is also shown. InSeat Solutions, Inc. manufactures a larger version of these inertial shakers and has used them in a wide variety of seat applications that center on massage therapy. Inertial shakers are relatively inexpensive (\$3.00 US per tactor). 


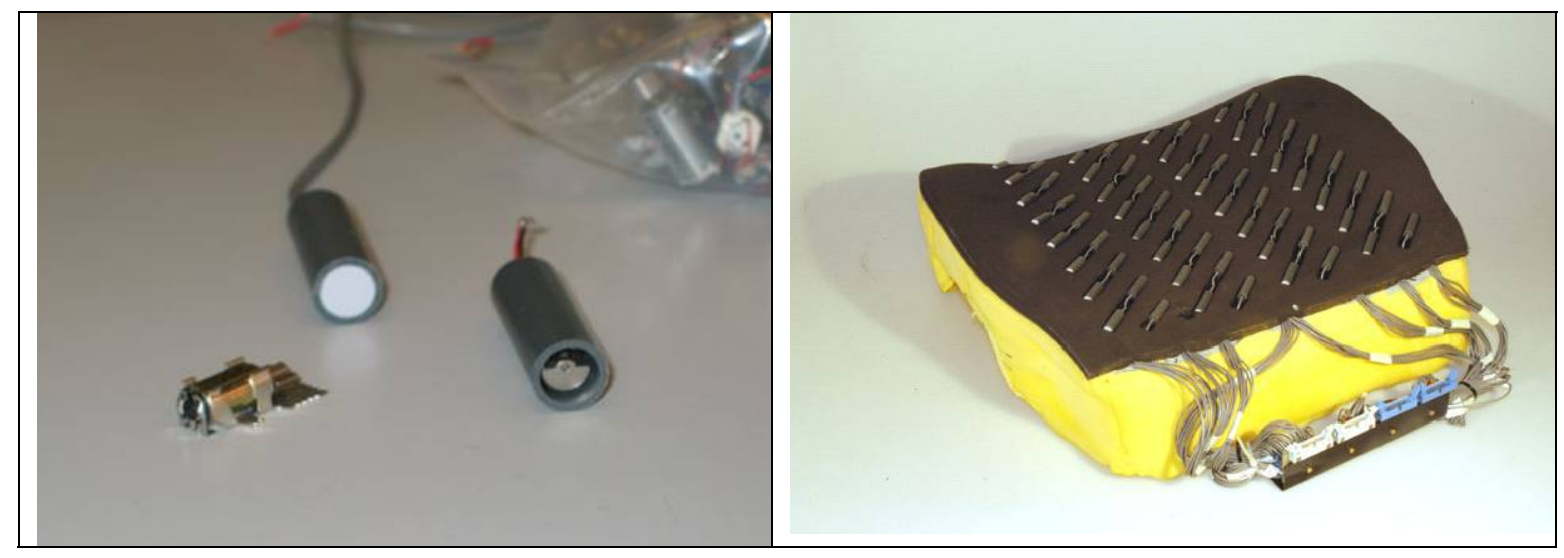

Figure 6. Left side: An eccentric mass tactor. Right side: 64 eccentric mass tactors positioned in a foam driver seat. Reproduced with permission from Fitch, Kiefer, Hankey and Kleiner (2007). Copyright 2007 by the Human Factors and Ergonomics Society. All rights reserved.

\section{Linear Actuators}

For linear actuators, the part of the tactor that touches the user's skin, or contactor, is the only part of the device that moves (Figure 7). The contactor is free to oscillate through a hole in the tactor housing, whose rigidity bears the weight of the user. The manufacturers claim that this design makes the tactor operation independent of loading. Since the amount of skin displacement is affected by the size of the contactor, they are optimally designed to be under $10 \mathrm{~mm}$ in diameter. Linear actuators are excellent for delivering precise tactile stimulation. They also allow frequency and intensity to be independently controlled. Placing numerous tactors adjacent to each other allows delivery of complex vibrotactile patterns. However, such tactors are only effective if the skin is directly contacting it, which prevents them from being an obvious device for a haptic seat since drivers typically change their posture while driving. Engineering Acoustics, Inc. (www.eaiinfo.com) manufactures a linear actuator that has been used inside vests (Craig, Jennings, Cheung, Rupert, \& Schultz, 2004) and belts (Cholewiak, Brill, \& Schwab, 2004). The C-2 tactor they manufacture is designed to optimally operate at $250 \mathrm{~Hz}$, but can be set to vibrate a wide ranger of frequencies. They have also developed tactors to optimally vibrate at lower frequencies. At the current time, their linear activator tactors are relatively expensive ( $\$ 150.00$ to $\$ 300.00$ US per tactor). 


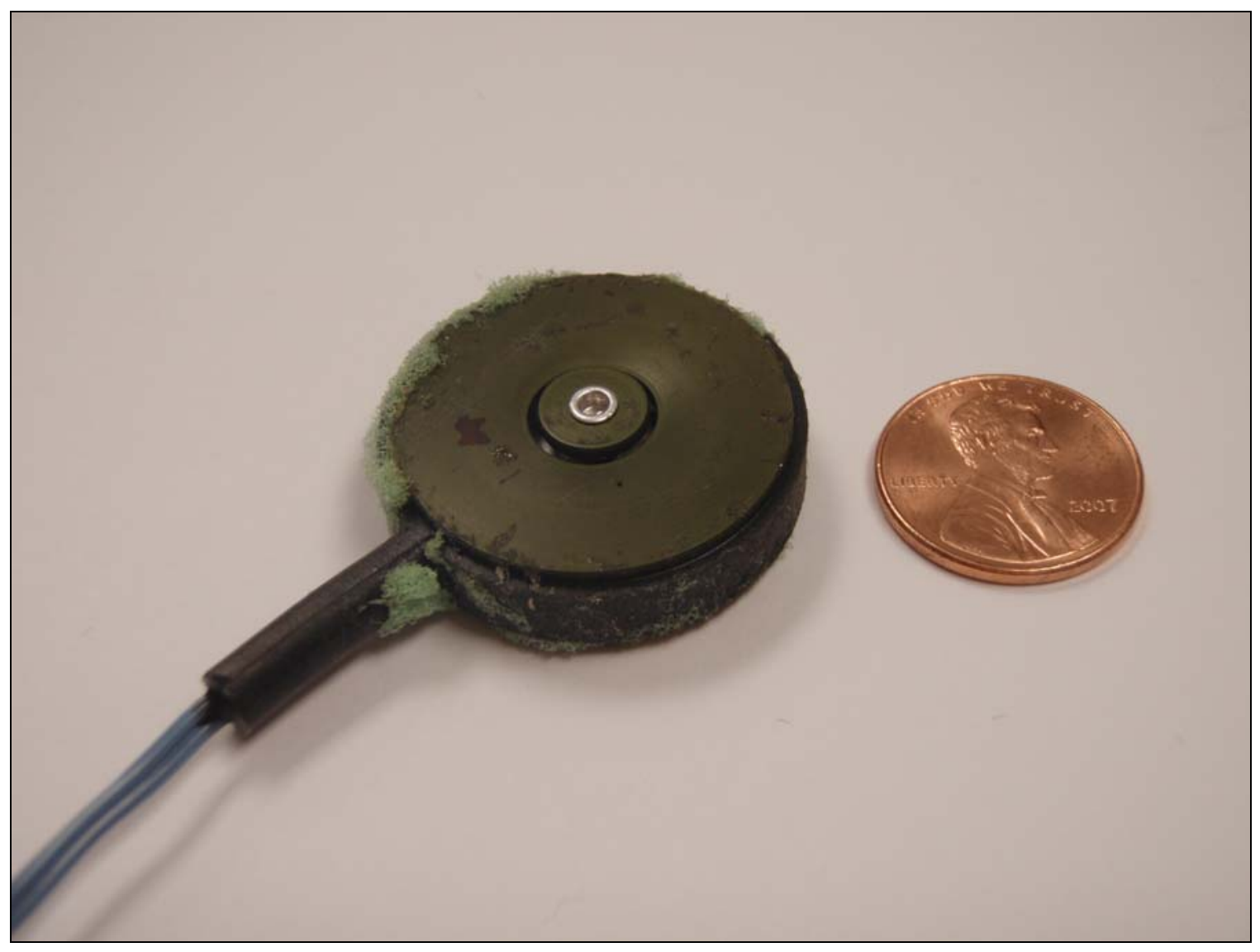

Figure 7. A linear actuator tactor.

\section{Pneumatic Tactors}

Pneumatic tactors deliver tactile feedback by rapidly compressing and releasing air inside a latex bladder (Figure 8). This pulsation of air delivers a distinct tapping sensation to the user. Steadfast Industries manufactures an inexpensive pneumatic tactor (\$5.00 US), but it requires a pulsed air source to drive them and it can be expensive. There are also limitations on tubing length and diameter, but they are markedly light and strong. 


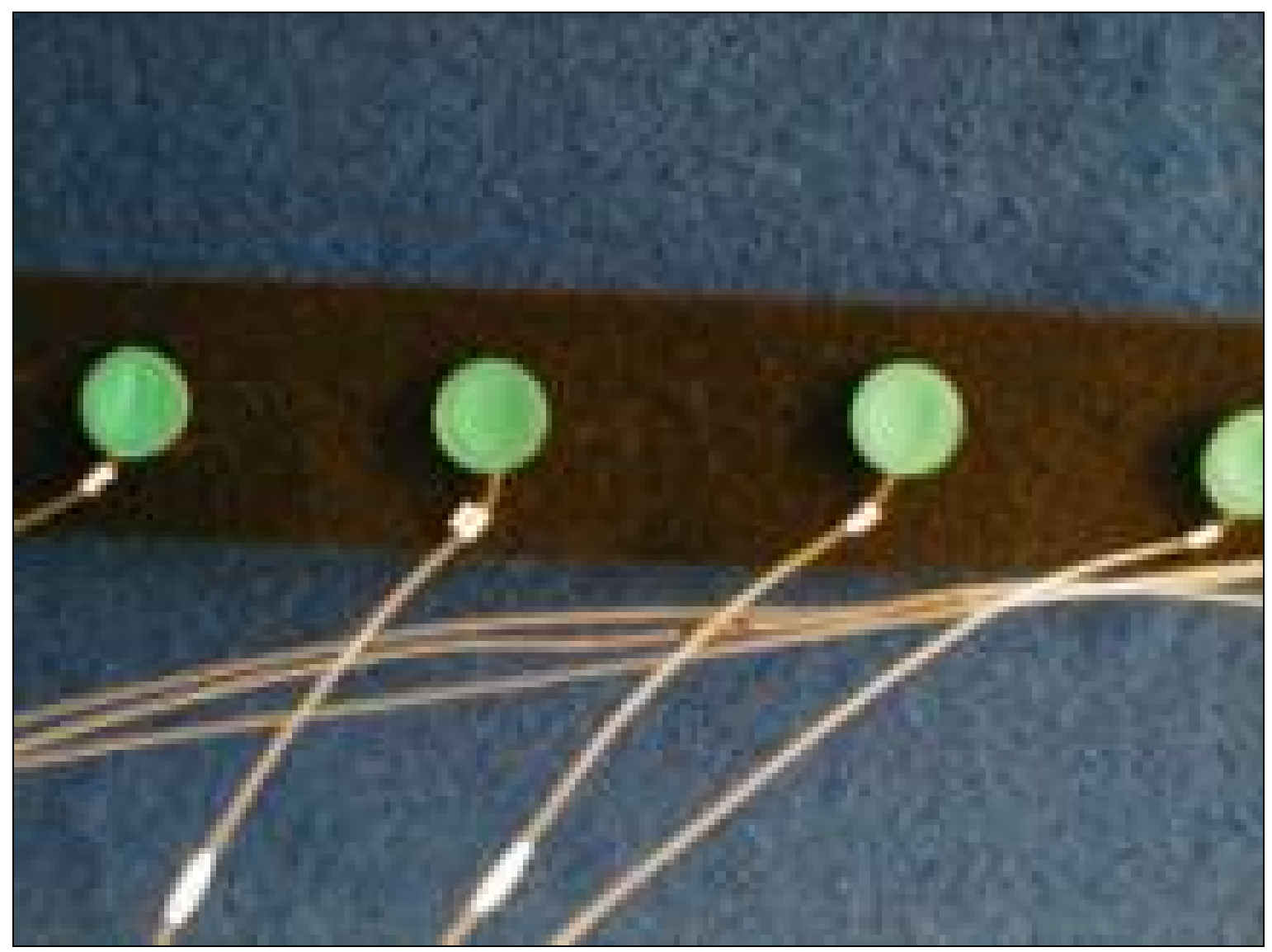

Figure 8. Four pneumatic tactors attached to a belt. Reproduced with permission from Dr. Roger Cholewiak's website (http://www.princeton.edu/ rcholewi/TRLTactorArrays.html)

\section{Tactor Placement}

The positioning of tactors in the seat pan affects the vibrotactile display's ability to communicate directional alerts to the driver. Designers need to consider three issues in developing haptic seat displays: 1) the driver's frame of reference, 2) changing driver posture, and 3) body anchor points.

\section{Frame of Reference}

The conveyance of direction requires two points, the point of contact by the tactor and the human body's frame of reference (Van Erp, 2005). The specified direction is the vector running between these two points. Van Erp (2005) suggests that the human body possesses two internal frame of reference points, one for each body half, when perceiving directions generated by tactors mounted in a belt around the user's waist. Figure 9 shows the directions perceived in response to 15 tactors arranged around the midsection of the body. Participants indicated the perceived direction to the tactors by standing in the middle of a horizontal plane and positioning a remotely controlled illuminated cursor around it. Figure 9 shows that the vectors passing through tactor locations and perceived 
directions coincide on both the left and right hip. Van Erp (2005) reports that the variability of responses depends on the tactor location, being higher for the left and right locations, and lower for the front and back locations.

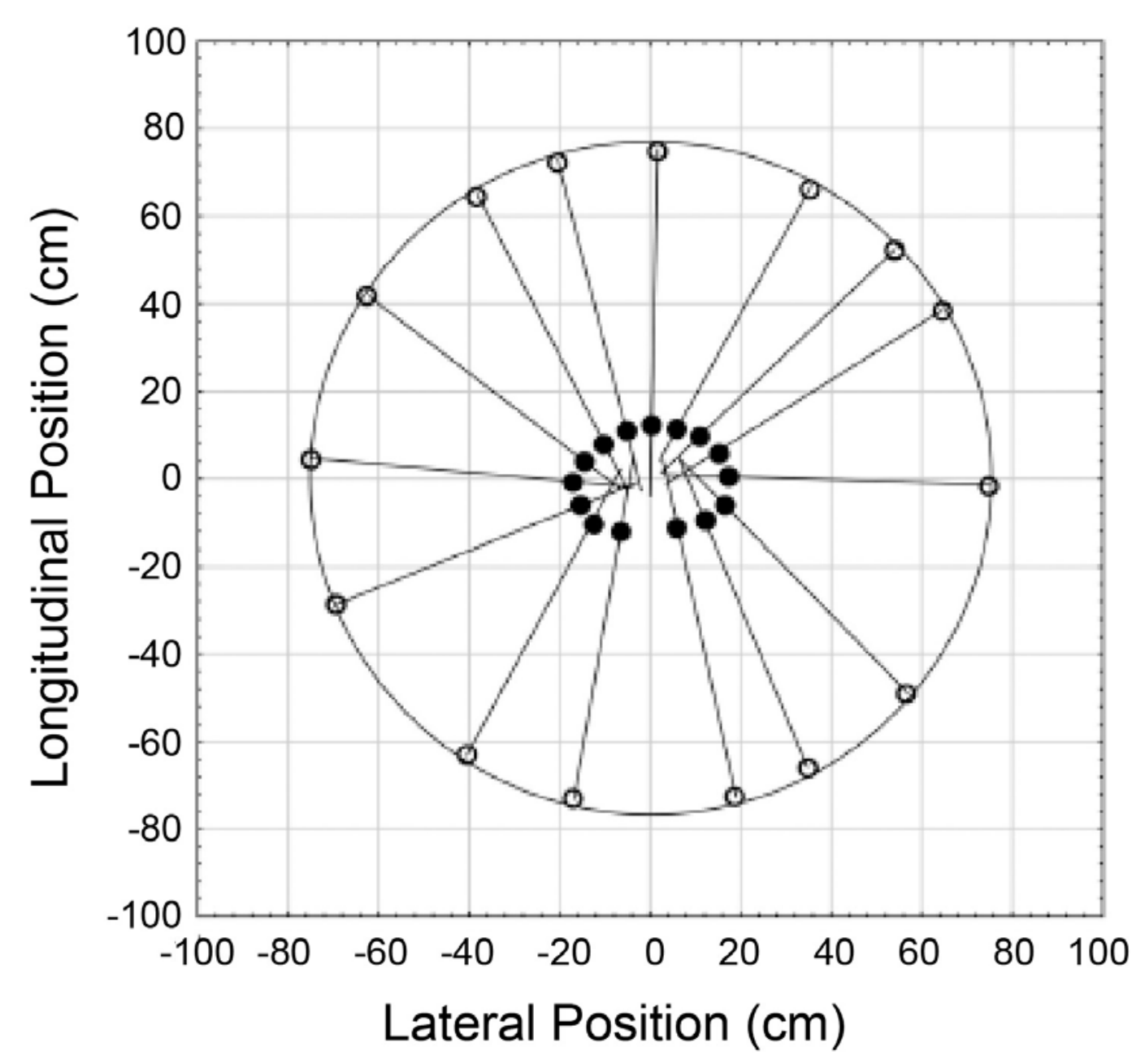

Figure 9. Average perceived directions in response to 15 tactors mounted around users waists via a belt. Reproduced with permission from Van Erp (2005). Copyright 2005 by Taylor and Francis Group (http://www.informaworld.com). All rights reserved.

Failing to consider the body's internal reference points can generate confusion when conveying a direction. In prototyping a vibrotactile vest designed to inform helicopter pilots of lateral drift (both velocity and acceleration) when hovering, McGrath, Estrada, Braithwaite, Raj, \& Rupert (2004) observed that displaying leftward acceleration cues on the right side of the body confused pilots. This suggests that directional haptic cues should be presented on the side of the body associated with the direction.

The location of drivers' frame of reference points while seated is unknown. However, 
since the hips remain in the same position relative to the head when seated, it is plausible that the reference points when seated are the same as when standing. Directional haptic seat designs presented in the literature vary on their consideration for drivers' reference points. The directional haptic seat investigated by Fitch, Kiefer, Hankey, et al. (2007) communicated directions using tactors located on the edges of the seat pan. Drivers were observed to localize haptic alerts presented through these tactors with $86 \%$ accuracy. The haptic seat tested by Lee et al. (2004) also used tactors placed in the front and side bolsters of the seat to communicate left, right and front directions. Although haptic alert comprehension is not specifically reported, drivers rated the haptic display as appropriate for communicating crash threats. Kochhar and Tijerina (2006), on the other hand, take a different approach to positioning haptic seat tactors. Although left and right directions (for lane change and lane departure warnings) are presented on the left and right edges of the seat pan, the directional curve speed warnings are generated by tactors on both sides of the body (an L-shaped pattern which consists of vibrating one side of the seat pan followed by the opposite side). Such warnings were reported as confusing, and were poorly associated with directional curve speed warnings. Additionally, forward collision warnings were generated by activating all the tactors in the seat (including those mounted in the seat back) at once. They report that such alert patterns may be useful for a general crash alert. Doing so, however, may have jeopardized the mapping of edge tactors with a respective direction.

Tan, Gray, Young, and Traylor (2003) developed a directional haptic seat that consisted of a $3 \times 3$ tactor array mounted in the seat back. While seated in an office chair, participants received a vibrotactile stimulus on the side of their back associated with the direction they needed to look to detect a change in the visual scene displayed in front of them. Results show that detection time to scene changes decreased by an average of $41 \%$ $(1630 \mathrm{~ms})$ when the location of the tactor coincided with the side a visual scene change occurred. It was also found that reaction time increased by an average of $19 \%$ (781 ms) when the locations of the tactors and visual scene changes did not coincide.

\section{Changing Posture}

In placing tactors in the seat pan around drivers' reference points, designers must remember that peoples' posture can change while driving. Slouching, for instance, can shift the driver's reference points forward. Fitch, Kiefer, Kleiner, and Hankey (2007) found that the errors made in localizing haptic seat alerts were predominantly onelocation-back errors, meaning the haptic cues were perceived as indicating a direction that was further back then the display was intended to indicate. Since testing lasted over two hours, it is foreseeable that these errors may have occurred due to some drivers slouching in the seat. In addressing this issue, side warnings, such as lane change and lane departure warnings may be most effective if they are generated by activating tactors located in the back-corners of the seat pan. Fitch, Kiefer, Kleiner, et al. (2007) observed very few errors in these locations (Figure 10). This lack of confusion may facilitate quick and accurate comprehension of vibrotactile alerts. 


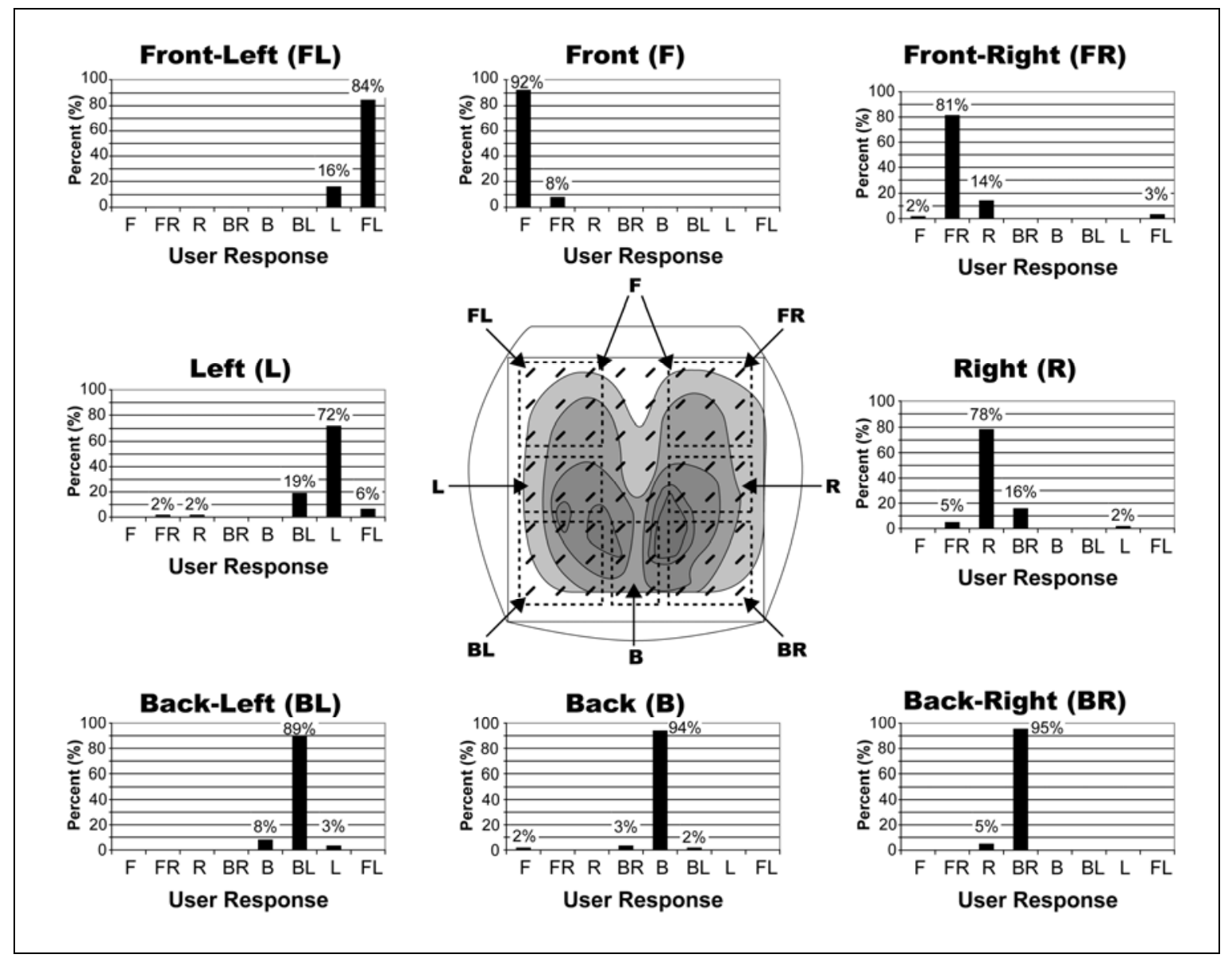

Figure 10. Center image: the $8 \times 8$ tactor array embedded in the driver seat pan. Tactors are represented by bold diagonal lines. The target locations are denoted by the dashed line borders. A seat pan pressure distribution plot is also shown (where darker areas indicate increased pressure). Peripheral graphs: the localization scores for each target location (front (F), front-right (FR), right (R), back-right $(B R)$, back (B), back-left (BL), left (L), and front-left (FL)). Note: the front target location was generated by simultaneously activating the FL and FR target locations. Reproduced with permission from Fitch, Kiefer, Kleiner, et al. (2007). Copyright 2007 by the Human Factors and Ergonomics Society. All rights reserved.

Another driver posture change occurs when drivers bring their feet close to the seat (such as when driving with the cruise control on). Doing so lifts their thighs off the front edge of the seat pan. Front edge tactors may therefore lose contact with the driver, which can be especially problematic for haptic seat displays that only use the front tactors to indicate forward warnings. Designing redundancy into these tactor locations will minimize the risk of missed alerts. Vibrating a large area along the front edge of the seat pan could allow the mid-thighs to sense the vibrations and allow the front direction to be communicated to the driver. Inertial shakers may be an appropriate tactor design in this case. Their diffuse vibrations are what allow vibrating cell phones stored inside a bag to be felt. 
To compensate for changing postures, some haptic seats have tactors placed in both the seat pan and the seat back. For instance, Kochhar and Tijerina (2006) observed that tactors placed in the seat back were intuitive at indicating a blind-spot warning to participants. However, the small of the back has been reported to be very sensitive. LeBlanc et al. (2006) report that last minute changes to a haptic seat designed to alert drivers of roadway departures were made before commencing a field operational test. Tactors originally located in the seat back were removed because some drivers felt they were "unsettling."

\section{Body Anchor Points}

It has been reported that certain parts of the body (typically joints such as the wrist, elbow, and shoulder) act as anatomically defined anchor points that serve as referents in vibrotactile localization. Cholewiak, Brill, and Schwab (2004) observed that localization performance of vibrotactile stimuli around the abdomen was greatest at the navel and the spine. This finding is of interest since all areas tested were found to have equivalent detection thresholds. Participants were able to easily eliminate these locations as possible responses to vibrotactile stimuli occurring close by. When the anchor points were removed from the test conditions (attained by rotating the belt so that the tactors no longer lined up along the naval and spine), overall localization performance decreased. Cholewiak et al. (2004) do not explain what makes certain body locations anchor points.

The presence of anchor points under the thighs has not been studied. However, the near perfect localization performance ( $94 \%$ correct) to tactors located in the middle of the back edge of the seat pan observed by Fitch, Kiefer, Hankey, et al. (2007) may be indicative of an anchor point located above the tail bone. Despite accurate localization in this area, startle responses are great and may interfere with drivers' ability to perform avoidance maneuvers. Directional haptic seats should use other tactor locations to indicate back directions, such as simultaneously activating the back-left and back-right locations or activating tactors located in the seat back.

Other anchor points include anthropometric edges. In response to other studies that were claiming poor localization performance of a vibrotactile forearm display, Oakley et al. (2006) investigated the feasibility of a 3 by 3 band of tactors on the top of the forearm. This location differed from the display used by Cholewiak and Collins (2003) which was fitted to the base of participants' forearms. Localization performance of each of the nine tactors averaged at $46 \%$. Yet, when localization performance was analyzed using just rows and columns ( 3 rows across the forearm, and three columns along the forearm, which would be parallel to a watch band), localization along the rows was $53.89 \%$ correct, while localization along the columns jumped to $84.40 \%$ correct. Cholewiak and Collins (2003) suggest the edges of the forearm assist people differentiate tactor columns from each other. The authors conclude that forearm vibrotactile displays that leverage body landmarks (such as the forearm edges) can yield localization performance comparable to that found with a torso display. 


\section{Number of Tactors}

How many tactors to place in a directional haptic seat is a profound question that has theoretical and practical implications on driver performance. Drivers are band-limited with respect to the amount of information they can process at once (Wickens \& Hollands, 1999). The Hick-Hyman Law (Hick, 1952; Hyman, 1953) suggests that as the number of tactors increases, where each tactor presents a new alert, driver response time to the alerts will increase. Miller's findings also suggest that driver's become progressively worse at correctly identifying alerts as the number of tactors is increased. When Cholewiak et al. (2004) investigated people's ability to localize 15 tactors mounted around the abdomen, they found that as the number of tactors presented in a condition decreased from 12 , to 8 , to 6 , localization accuracy dramatically improved from $74 \%$ to $92 \%$ to $97 \%$ correct. Here, each tactor was activated on its own. This study demonstrates the effects of increasing the amount of information conveyed in a display on human performance.

From a practical standpoint, a pattern that activates multiple tactors at the same time will increase stimulus intensity since more receptive fields are stimulated at the same time (Oakley, et al., 2006). Larger vibration areas also increase the alert's redundancy and allow for changing diver posture. In this case, it is not the number of tactors that dictate information transmission rate, but rather the number of patterns used in the haptic display vocabulary.

\section{Inter-Tactor Distance}

It was previously discussed that people are physically limited in their ability to resolve the separation of two vibrotactile stimuli. Tactors positioned too closely to each other can become indistinguishable. Cholewiak et al. (2004) observed localization performance decrements as tactor spacing around the abdomen increased. Participants were more accurate at localizing 8 tactors positioned around their entire trunk than localizing 7 tactors positioned around half of their trunk. Here, performance decrements were observed despite the reduction in the amount of information transferred ( 7 instead of 8 tactors).

Tactor separation is a concern since vibrotactile CAS alerts that are confused with another may potentially delay driver responses. Sayer, Sayer, and Devonshire (2005) used a directional haptic seat consisting of four tactors to investigate a CSW and a LDW alert. The CSW alert was generated by a steady vibration in the front-left and front-right tactors, while the LDW alerts were generated by vibrating the front-left and back-left tactors to signal a left lane departure, and vibrating the front-right and back-right tactors to signal a right lane departure. Because LDW and CSW alerts shared common tactors, some participants were unable to differentiate them. Sayer et al. (2005) report that some participants commented that they felt as if "the whole seat buzzed" and that they "couldn't tell which leg was being vibrated." The directional haptic seat localization errors reported in Fitch, Kiefer, Hankey, et al. (2007) also indicate small inter-tactor distances can lead to confusion. Although each pattern used mutually exclusive groups of tactors, the display had an inter-tactor distance of $4 \mathrm{~cm}$. It is possible that confusion 
can be minimized by increasing the spacing between tactors and not using the same tactors to indicate different alerts.

\section{Stimulus Frequency}

Designers need to be careful when selecting tactor frequency for vibrotactile seat displays. If the frequency is too high, the vibrotactile cue may be perceived as too sharp. If the frequency is too low, it may be masked by naturally occurring vibration from the road. An idea of which frequencies are appropriate can be gathered by citing the literature. Fitch, Kiefer, Hankey, et al. (2007) presented haptic seat messages concentrated between $100 \mathrm{~Hz}$ and at $140 \mathrm{~Hz}$. Drivers rated these vibrotactile stimuli as not annoying (Fitch, et al., 2005). Van Erp and Van Veen (2004) presented tactors mounted in the seat pan at $250 \mathrm{~Hz}$. Subjective ratings of these alerts, however, were not reported. Ho, Tan, and Spence (2005) activated two tactors mounted around the waist at 290 Hz. Van Erp, Van Veen, Jansen, and Dobbins (2005) also experimented with tactors mounted around participants' waist. For this experiment, they presented tactors at 160 Hz.

\section{Stimulus Intensity}

The coding of stimulus intensity to haptic alerts must be carefully considered. This is because vibrations that are too intense may be perceived as annoying, and vibrations that are too soft may not be perceived at all. Vibrotactile stimulus intensity is currently used to provide haptic feedback in video games. For instance, Major League Baseball 2007 for the Xbox 360 uses increased vibration intensity in the controller to notify users when the region selected to pitch the ball extends past the strike zone. In general, it is believed that no more than three tactor intensities should be used for one communication dimension (Geldard, 1960; Sachs, Miller, \& Grant, 1980). Lee et al. (2004) followed this design recommendation when investigating a haptic driver seat. The tactors used vibrated at three intensity and frequency levels (severe, moderate, and negligible) to indicate the threat level of lead vehicles in a driving simulator.

\section{Stimulus Duration}

The length of the vibrotactile stimulus affects how it is perceived. People are poor at perceiving short duration vibrotactile stimuli. Sensitivity improve as stimulus duration lengthens (Perez \& Weed, 1991). As pulse duration increases, however, it reaches a region where sensitivity no longer improves. The length of the vibrotactile stimulus can differentiate alerts. Some cell phones, for instance, differentiate voice mail messages from low battery messages by vibrating the phone for a longer period of time. In the context of collision avoidance displays, however, this parameter may reduce the effectiveness of the alerts if drivers must wait for the warning to complete in order to comprehend it. Vibrotactile CAS alerts should be designed to be quickly differentiable and have the same duration. 


\section{Stimulus Rhythm}

Stimulus rhythm can be an effective parameter for differentiating vibrotactile alerts (Lorna, Stephen, \& Helen, 2005). Lorna et al. (2005) showed that people were able to accurately identify three vibrotactile messages that differed by rhythmic patterns (greater than $90 \%$ correct). The rhythms differed in the number of pulses generated per message. The tempo of the pulses is also an effective component of rhythm. Cell phones rely on vibrotactile stimulus rhythm to communicate different types of messages. These one dimensional vibrotactile displays use dash (steady activation of tactor), dot dot (bursts of tactor activation similar to pulses in auditory alerts), and dot dash (one short burst followed by one longer vibration). For two-dimensional vibrotactile displays where tactors are arranged in a grid, more complex patterns can be generated. For instance, Van Erp and Van Veen (2004) communicated distance to an upcoming turn by using three vibrotactile temporal patterns. The patterns used consisted of multiple vibrations with a burst length of $60 \mathrm{~ms}$. The longest distance cue presented bursts every $270 \mathrm{~ms}$. The medium and shortest distance cues, however, used a sweeping pattern that sequentially activated four tactors under the driver's leg in decreasing intervals. The medium distance cue used intervals that decreased from $270 \mathrm{~ms}$ to $60 \mathrm{~ms}$, while shortest distance cue used intervals that decreased from $60 \mathrm{~ms}$ to $10 \mathrm{~ms}$. This haptic seat design was found to reduce mental workload when operating an in-vehicle navigation system.

\section{HAPTIC DISPLAY DESIGN CONSTRAINTS}

A design goal when developing haptic seat displays should be to keep their bandwidth low (Boff \& Lincoln, 1988). This allows the driving population to accurately comprehend the alerts. The $86 \%$ correct localization performance to directional haptic seat alerts observed by Fitch, Kiefer, Hankey, et al. (2007) can be criticized for being inadequate. However, these alerts were localized without an established crash threat context. Successful CASs will yield near perfect alert comprehension. The Engineering Data Compendium (Boff \& Lincoln, 1988) proposes design constraints set attain this level of information transfer.

- Because stimulation of the skin involves activation of many receptor systems, assumptions regarding the underlying populations of structures contributing to a sensation should be made with caution.

- The magnitude of prolonged stimulation will suffer from adaptation after several minutes of continued presentation.

- Display systems using vibration frequency for encoding parameters of the display should limit the number of frequencies to fewer than seven to nine and employ changes in frequency greater than $20 \%$. If the display system depends on intensity changes, the number of levels should be limited to four.

- The level of thermal adaptation in the skin will affect judgments of rate of vibration as well as vibrotactile thresholds.

- Individual differences for vibrotactile sensitivity are great.

- Practice can increase tactile acuity. 


\section{CHAPTER 3. EXPERIMENT 1: EFFECTS OF INCREASING THE NUMBER OF CAS ALERTS ON DRIVER PERFORMANCE}

\section{PURPOSE}

Active safety systems that warn drivers of impending collisions have been developed. How these systems alert drivers when integrated, however, is a crucial component to their effectiveness that hinges on the consideration of human factors. Drivers must be able to quickly perceive, process, and execute the correct maneuver in response to the alerts in order to avoid a collision. Active safety system designers should question whether drivers can efficiently process multiple alerts. Information Theory (Shannon \& Weaver, 1949) states that the amount of information conveyed by an event is a function of: 1) the number of events that can occur, 2) the probability that an event will occur, and 3) the sequential constraints imposed on the occurrence of an event (Wickens \& Hollands, 1999). Hick (1952) and Hyman (1953) applied Information Theory to explain human performance in a choice response task. In doing so they established the Hick-Hyman Law, which states that the more information an event conveys, the longer humans take to process it. Furthermore, Miller (1956) applied Information Theory and discovered that the span of immediate memory is limited to a finite amount of information. Humans can process 2 to 3 bits of information, or $7 \pm 2$ chunks of information, before performance significantly degrades. In applying the Hick-Hyman Law and Miller's Number to active safety system design, it is foreseeable that drivers' response performance (response time and accuracy) to any one alert may degrade as the number of active safety systems that generate alerts increases. Here, the additional attentional resources required to process which alert is presented, and which response is appropriate, translates into lengthened response times and potentially more mistakes. The purpose of this experiment was to quantify the effects of increasing the amount of information conveyed by a haptic driver seat on drivers' response performance. Although various alert modalities have been proposed for the use in active safety systems, alerts solely presented through a haptic seat were an interest owing to results obtained in previous research (Fitch, Kiefer, Hankey, et al., 2007).

\section{METHOD}

Since participants performed Experiments 1, 2 and 3 in one session, aspects of the methods are common to all three studies. The selected participants, the test vehicle used, and the haptic driver seat are described in this experiment, but apply to all three experiments. Participants took 2.5 hours to complete all three experiments. The order of the experiments was always Experiment 1 first, followed by Experiment 2, and ended with Experiment 3. This was done because there was an interest in isolating participant's exposure to Experiment 1. Drivers were thus familiar with the apparatus when they performed Experiments 2 and 3. 


\section{Participants}

Twenty-four participants were equally selected from two age groups: a younger age group consisting of drivers between the ages of 18 and 25 years old, as well as an older age group consisting of drivers over the age of 65 years old. An equal number of males and females were selected. Participants had to have a minimum visual acuity of 20/40 to participate. Participants were screened for lingering effects of neck/spine injuries/pain, heart/cardiovascular conditions, history of a stroke, brain tumors, head injuries, and motion sickness. Participants also could not participate if they had a recent occurrence of a respiratory disorder, dizziness, vertigo, or other balance problems, inner ear problems, migraine and tension headaches, epileptic seizures, diabetes, advanced Osteoporosis, eye injuries or retinal detachment. Participants also had to have a valid driver's license to be eligible. The telephone script used to screen participants is presented in Appendix A. Participants were informed that video and audio recordings would be made of them throughout the study. Participants indicated on their informed consent form whether they allowed their footage to be used for research reporting purposes.

\section{Apparatus}

\section{Test Vehicle}

A 2002 Cadillac STS was used in this study (Figure 11). Participants were able to adjust the position of the driver seat, lumbar support, and the steering wheel to their liking. A data acquisition system (DAS) was installed in the trunk of the vehicle. The DAS was controlled via a keyboard and monitor assembled together in the back seat of the vehicle. Video footage of the forward roadway, brake pedal, driver's face and the driver's hands placed on the steering wheel were recorded with cameras approximately $1 \mathrm{inch}^{3}$ in size that were indiscreetly mounted in the vehicle cabin. Video footage was digitally recorded through a quad view at $29.97 \mathrm{~Hz}$ (Figure 12). Advancing through the video footage frame-by-frame post experiment allowed for precise determination of when a driving maneuver began and ended. An audio recording also captured the participant's verbal responses. To assist with response scoring, the in-vehicle experimenter entered the participant's response with the keypad. However, all marked responses were verified post-hoc. The DAS was able to record pertinent vehicle variables such as speed, the brake pedal position, throttle position, and acceleration. The data was digitally recorded on a hard-drive, where it was downloaded to a secure server after each experimental session. 

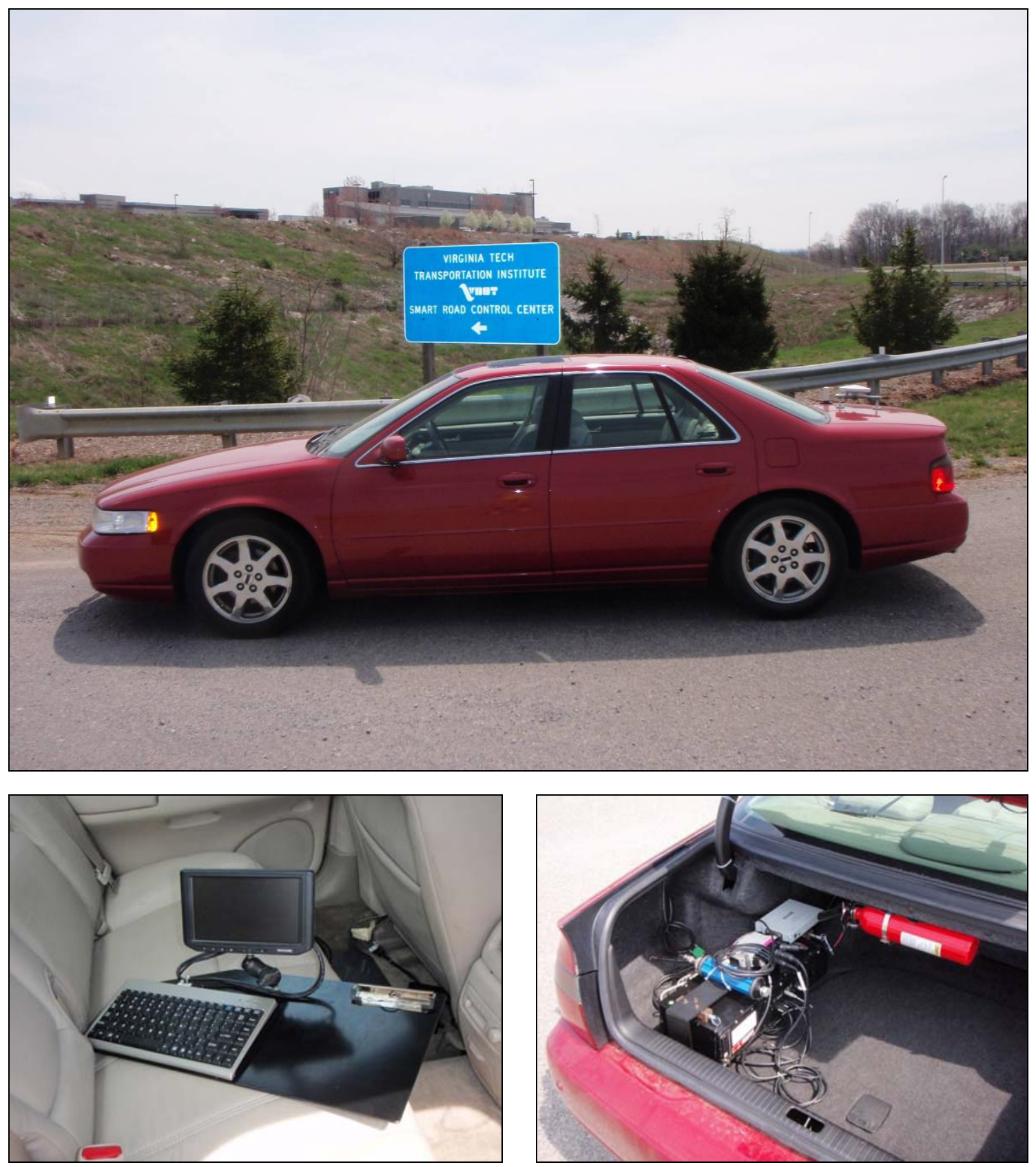

Figure 11. The 2002 Cadillac STS test vehicle. Counterclockwise starting from the top: a) profile photograph of test vehicle, b) the data acquisition system, c) in-vehicle experimenter's computer to control the presentation of the haptic seat alerts. 


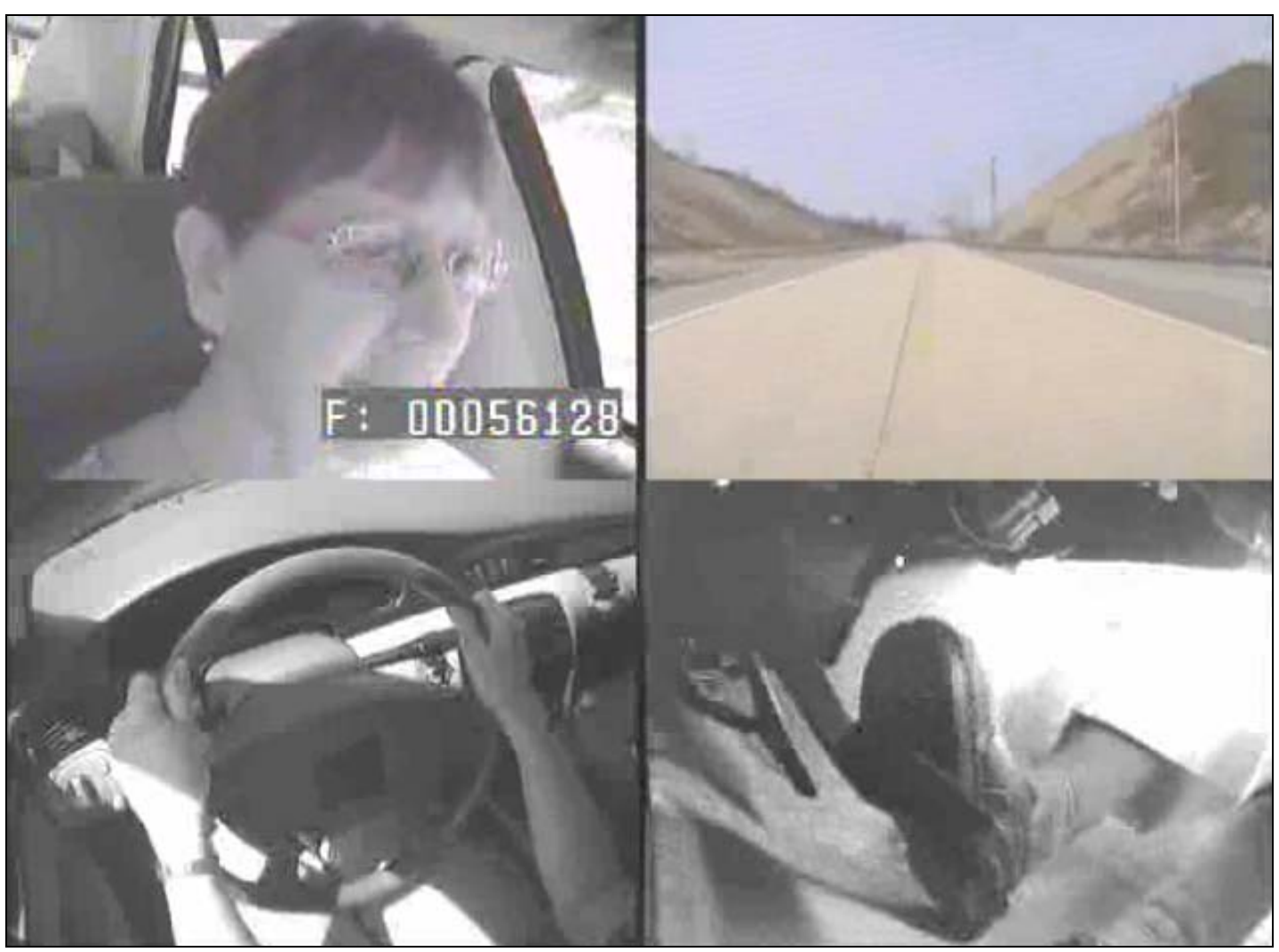

Figure 12. Quad view of recorded video footage.

\section{Haptic Driver Seat}

Inertial shaker tactors manufactured by InSeat Solutions, LLC.

(www.inseatsolutions.com) were used in this experiment. Each tactor consisted of a small DC motor mounted on a metal plate. The motor rotated an eccentric cam shaft causing the motor-plate combination to vibrate. The integral dimensions of vibration frequency and intensity were controlled by varying the motor speed, where faster speeds increased the vibration frequency and intensity. The motor's speed was controlled through pulse width modulation of the DC voltage applied to the tactors. The signal's duty cycle controlled the effective voltage, and thus the haptic stimuli's frequency and intensity. The duty cycle was divided into 100 steps, allowing the intensity to be controlled in one percent increments. Each step was $1.7361 \mu$ s long. A duty cycle of 50 percent was used in this experiment. Unfortunately, equipment to measure the actual frequency in which the cam makes a complete revolution was not available. Discussion with InSeat Solutions, LLC. revealed that this specification was unknown to them as well. Furthermore, it should be noted that the tactors' nominal frequency changes when they are loaded by the driver's weight. The tactors were programmed to vibrate at an intensity that was clearly perceptible without being overly annoying. It should be noted that not one driver failed to detect an alert. The interested reader is referred to Append $G$ for the results of drivers' subjective ratings of the alerts' intensity and annoyance.

The driver seat was outfitted with six inertial shaker tactors (Figure 13). Two tactors 
were mounted along the front edge of the driver seat (Figure 14). Two more tactors were mounted in the back-left and back-right corners of the seat pan (seat bottom). The final two tactors were mounted in the middle of the seat back. Experiment 1 only used the tactors mounted in the seat pan, while Experiment 2, which is presented in Chapter 4, investigated the use of tactors mounted in the seat back.
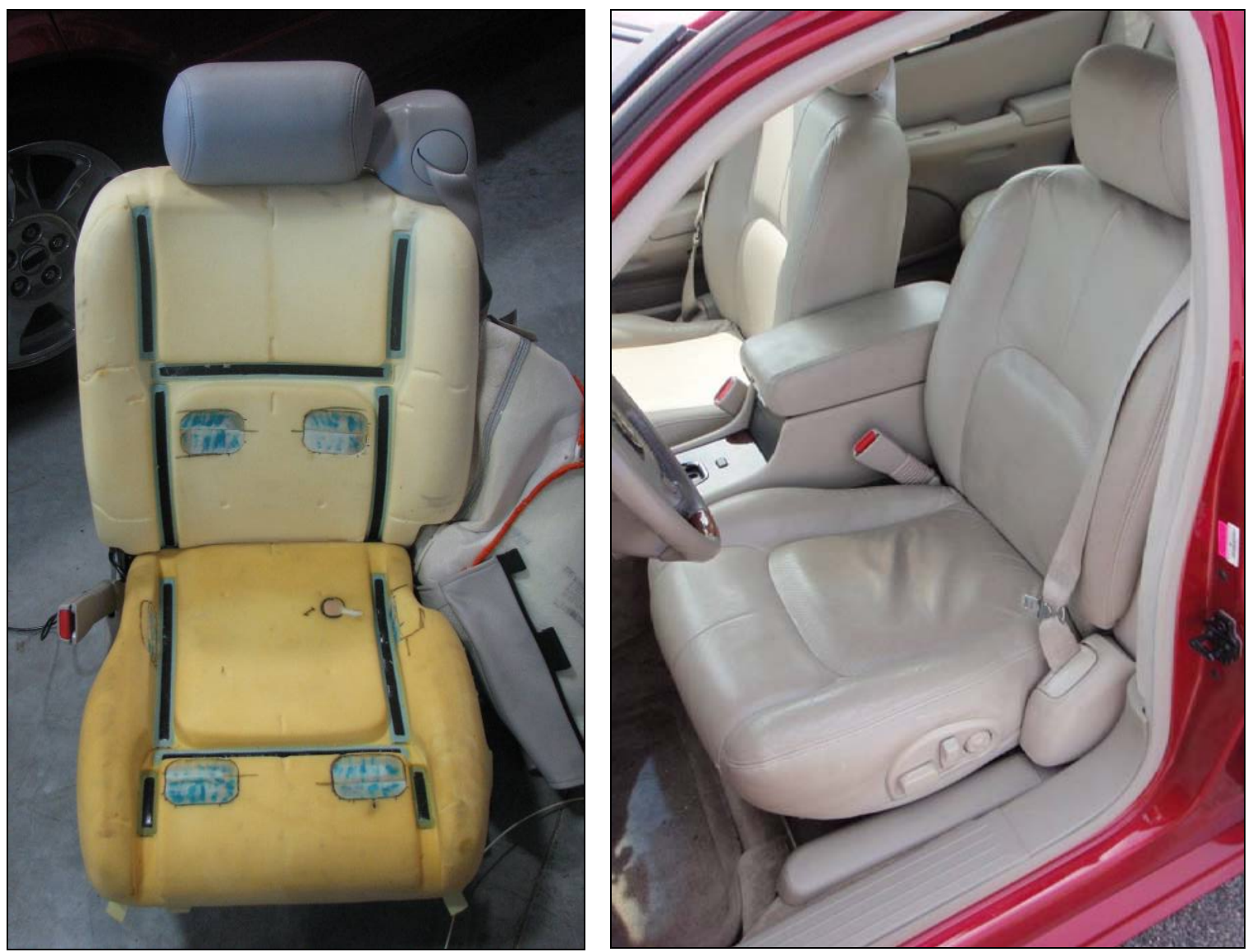

Figure 13. Mounting of the six inertial shaker tactors in the driver seat.
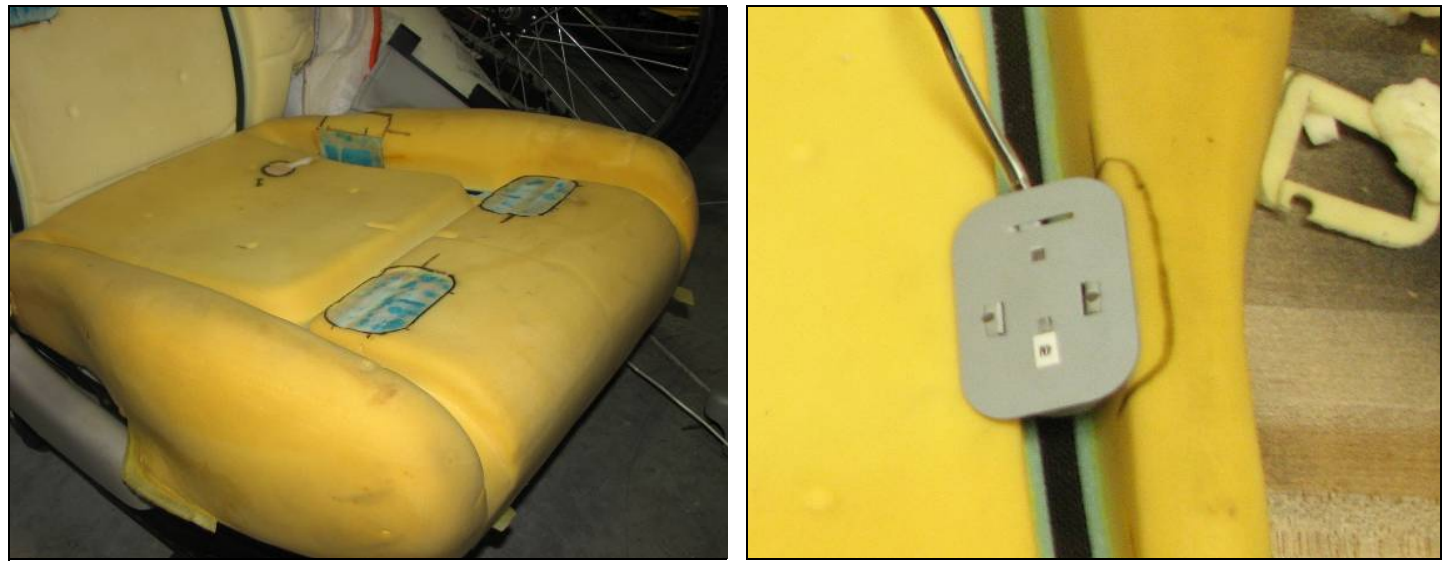

Figure 14. Close-up of the inertial shaker tactors manufactured by InSeat Solutions, LLC. 
The four seat-pan tactors were used to generate seven haptic alerts by combining three alert locations with three alert patterns. The three alert locations were the front, right, and left locations. The front location was generated by simultaneously vibrating the two tactors in the front edge of the seat. This ensured that drivers perceived the alerts using either their left or right leg (in case one leg was lifted off the seat, which can occur when using cruise control). These tactors were also mounted in front of the driver's frame of reference, which is believed to be located in their hips (Van Erp, 2005). The vector passing through these two points, which is directed forward, is believed to support cueing drivers' attention to the forward roadway. The left direction was generated by vibrating the back-left tactor. This location was designed to be clearly differentiable from the front location. This was accomplished by mounting the back-left tactor at the opposite end of the seat pan from the front tactors and by not vibrating any of the front tactors when generating the alert. The vector passing through the driver's frame of reference and this tactor is believed to support cueing drivers' attention to the left. In a similar fashion, the right location was generated using only the back-right tactor.

The three vibrotactile patterns used were the pulse pattern, the dash pattern, and the double pulse pattern. The pulse pattern consisted of briefly activating the tactors five times for $200 \mathrm{~ms}$ each. An inter-stimulus interval of $50 \mathrm{~ms}$ was used by this pattern. The dash pattern consisted of activating the tactors for $1000 \mathrm{~ms}$. The double pulse pattern consisted of activating the tactors for $200 \mathrm{~ms}$ on, $50 \mathrm{~ms}$ off, $300 \mathrm{~ms}$ on, $200 \mathrm{~ms}$ off, 200 $\mathrm{ms}$ on, $50 \mathrm{~ms}$ off, $300 \mathrm{~ms}$ on, and $50 \mathrm{~ms}$ off. Tactor activation for each pattern was controlled to be $1000 \mathrm{~ms}$.

The seven haptic alerts and the patterns used to generate them are presented in Table 2.

Table 2. Haptic Seat Alert Patterns

\begin{tabular}{|c|c|}
\hline Alert & Haptic Pattern \\
\hline Forward Collision Warning & $\begin{array}{l}\text { Front-left and front-right tactors simultaneously } \\
\text { activate five times ( } 200 \mathrm{~ms} \text { on, } 50 \mathrm{~ms} \text { off) (Pulse) }\end{array}$ \\
\hline Curve Speed Warning & $\begin{array}{l}\text { Front-left and front-right tactors simultaneously } \\
\text { activate for one second (Dash) }\end{array}$ \\
\hline Intersection Violation Warning & $\begin{array}{l}\text { Front-left and front-right tactors simultaneously } \\
\text { activate four times each ( } 200 \mathrm{~ms} \text { on, } 50 \mathrm{~ms} \text { off, } \\
300 \mathrm{~ms} \text { on, } 200 \text { off, } 200 \mathrm{~ms} \text { on, } 50 \mathrm{~ms} \text { off, } 300 \mathrm{~ms} \\
\text { on, } 50 \text { off) (Double Pulse) }\end{array}$ \\
\hline Left Lane Change Warning & $\begin{array}{l}\text { Back-left tactor activates five times ( } 200 \mathrm{~ms} \text { on, } \\
50 \mathrm{~ms} \text { off) (Pulse) }\end{array}$ \\
\hline Right Lane Change Warning & $\begin{array}{c}\text { Back-right tactor activates five times ( } 200 \mathrm{~ms} \text { on, } \\
50 \mathrm{~ms} \text { off) (Pulse) }\end{array}$ \\
\hline Left Lane Departure Warning & Back-left tactor activates for one second (Dash) \\
\hline Right Lane Departure Warning & Back-right tactor activates for one second (Dash) \\
\hline
\end{tabular}


Specific mappings were designed into the haptic seat. The FCW, CSW, and IVS alerts warned drivers of danger ahead. They were thus generated using the front location. Since drivers would press the brake pedal to avoid danger ahead, this location established one stimulus-response mapping between front alerts and pressing the brake pedal. Since the left LCW and LDW alerts warned drivers of danger on the left, they were generated using the left location. Since drivers would steer right to avoid danger on the left, this established another stimulus-response mapping. Similarly, since the right LCW and LDW alerts warned drivers of danger on the right, they were generated using the right location. Drivers would steer left to avoid danger on the right.

The vibrotactile patterns were also mapped to specific meanings. The alerts that warned drivers of conflicts with vehicles were generated using the pulse pattern, while the alerts that warned drivers that the vehicle was being poorly controlled were generated using the dash pattern.

It should be mentioned that the double pulse pattern was used in lieu of an alternating pattern as a result of pilot testing. The alternating pattern used the same pattern as the FCW alert, but alternated the activation of the front-left and front-right tactors. This pattern was discarded because it was difficult to distinguish front-left vibrations from front-right vibrations.

\section{Virginia Smart Road}

This experiment was conducted on the Virginia Smart Road (Figure 15). The Virginia Smart Road is a 3.54-km (2.2-mile) closed-course test track that was designed to facilitate research on Intelligent Transportation Systems (ITS), human factors and transportation safety, as well as road surface properties. The road is built to Virginia Department of Transportation and Federal Highway Administration standards. To ensure participant safety when conducting experiments, the Smart Road restricts public access and is monitored through video surveillance 24 hours a day, 7 days per week. A detailed listing of the Smart Road's capabilities is presented in Appendix B. 


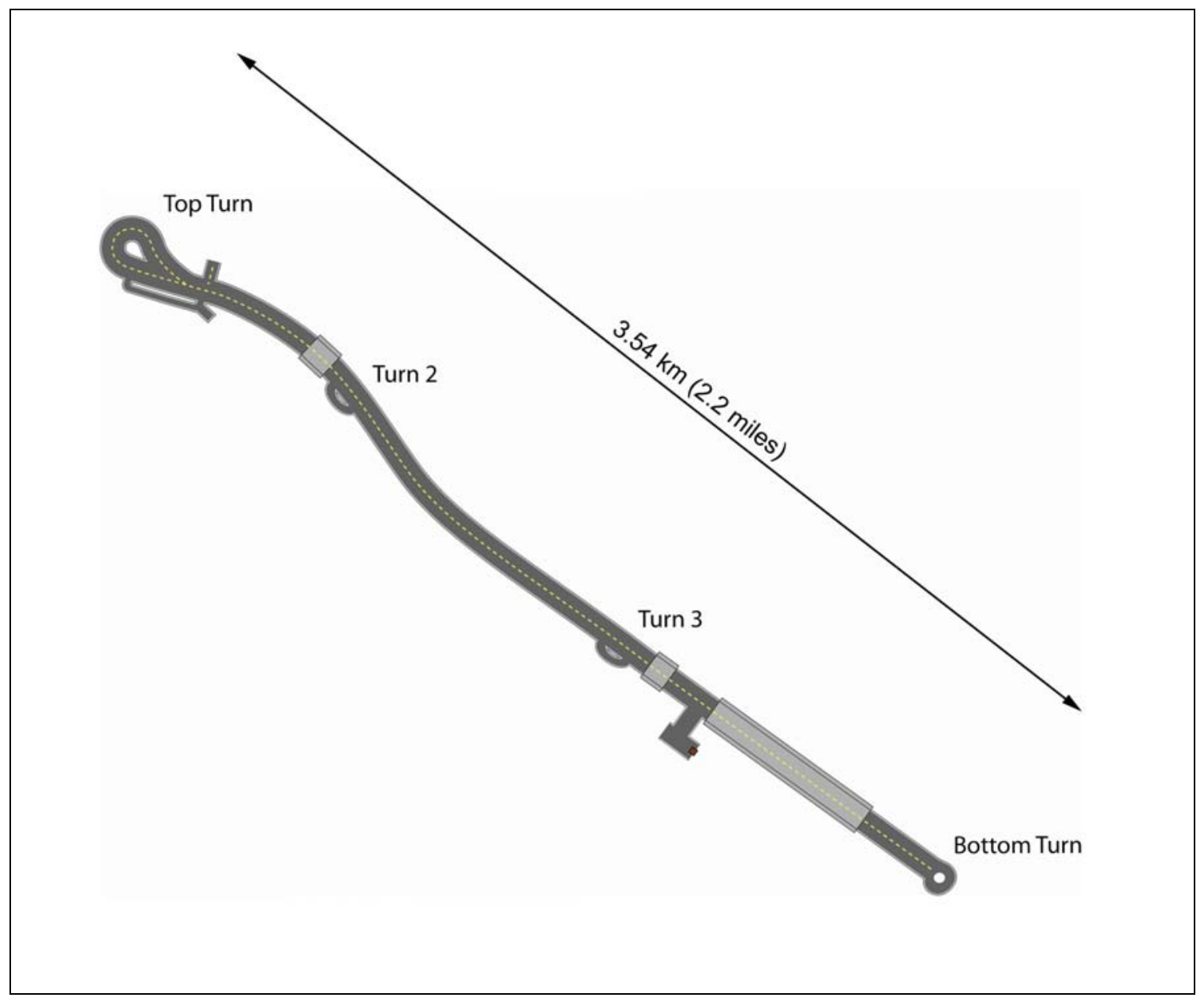

Figure 15. Diagram of the Virginia Smart Road.

\section{Procedure}

Upon arriving at the Virginia Tech Transportation Institute, participants were seated inside the subject-preparation room where they were given time to read and sign the study's Informed Consent form. The informed consent form, as well as the Virginia Tech's Institutional Review Board's signed approval letter, are presented in Appendix C. Participants showed their valid driver's license to the experimenter prior to continuing. The experimenter described the purpose of the study and the experimental tasks that the participant would be asked to perform. Participants then completed a Snellen eye exam, where they had to have 20/40 vision to participate. Participants' height and weight were also measured prior to heading out to the test vehicle.

Participants were asked to remove their wallets and cell phones prior to sitting in the test vehicle. Participants sat in the driver seat and were allowed to adjust the seat, steering wheel, and mirrors to their liking. The experimenter sat in the back-right passenger seat. The haptic driver seat and experimental tasks were explained while the vehicle was stationary. The participant then drove the vehicle out to the Virginia Smart Road. 
Participants were told that they would experience the seven vibration alerts during the study. Each alert was demonstrated to participants. For each alert, participants were told what the alert meant, as well as what the appropriate driving response should be.

Participants drove the test vehicle at $32 \mathrm{~km} / \mathrm{h}(20 \mathrm{mph})$ down the middle of the Virginia Smart Road. Using mark points on the road, the experimenter presented one of the seven haptic seat alerts to the driver. Only one alert was presented at a time. The participant's task was to correctly identify the presented haptic seat alert. This consisted of executing the proper manual response as fast as possible, and then verbally identifying the alert. The number of possible alerts that could be presented to drivers was controlled at three levels. The first level consisted of just the FCW alert. Driver response performance to this one alert was used to establish a baseline. The second level presented an FCW alert, a right LCW alert, and a left LCW alert. These three alerts were selected for the second level because they all used the same vibrotactile pattern (five pulses). The third level presented all seven alerts. The order in which participants were exposed to levels 1,2, and 3 was counterbalanced. Participants were instructed to apply the brakes if an FCW, CSW, or IVW alert was presented. They were instructed to steer into the right lane if a left LCW or left LDW alert was presented. Similarly, they were asked to steer into the left lane if a right LCW or right LDW alert was presented. After participants performed the manual response, they verbally identified the alert. Verbal responses were scored based on meaning, and not specific terminology. Participants returned the vehicle to the middle of the Smart Road after responding to an alert. Two practice trials per alert type were provided prior to the experimental trials. For each condition, each type of alert was presented six times. Therefore, the FCW alert was presented eight times to a participant when it was the only alert presented, 24 trials were presented when three alert types were used (i.e., eight trials for each alert type), and 56 trials were presented when seven alerts were used. Participants completed a questionnaire regarding the task difficulty after each condition. This portion of the experiment took one hour to complete. The protocol is presented in Appendix D.

\section{Independent Variables}

This experiment consisted of a 2 (Age) x 2 (Gender) x 3 (\#Alerts) mixed factors design. The independent variables are explained below.

\section{Age (Older Younger) - Between-Subjects}

Fitch et al. (2005) found that haptic seat alert localization performance degrades with age. Verrillo, Bolanowski, and Gescheider (2002) also report that the subjective magnitude of vibrotactile stimuli decreases with age. Since age effects were found in previous research, participants were selected from two age groups. Participants between the ages of 18 and 25 years old were selected for the younger age group. Participants above the age of 65 were selected for the older age group. 


\section{Gender (Female, Male) - Between-Subjects}

An equal number of females and males were selected from each age group for this experiment in order to generalize the results to the driving population.

\section{Number of Alerts (One Alert, Three Alerts, Seven Alerts) - Within-Subjects}

Three levels were used for the number of alerts independent variable.

1) One Alert

The single alert condition consisted of an FCW alert. Driver response behavior to one alert was used to establish a baseline.

2) Three Alerts

The three-alert condition consisted of the FCW alert, a right LCW alert, and a left LCW alert. The five-pulse vibrotactile pattern was common to these three alerts. They only differed by the location of the driver seat in which they activated. This was done to prevent the vibrotactile patterns from confounding comparisons of drivers' response performance to three alerts to their response performance to one alert.

\section{3) Seven Alerts}

In addition to the three alerts previously mentioned, the seven-alert condition used a CWS alert, an IVW alert, a right LDW alert, and a left LDW. These additional alerts were presented through areas of the driver seat that were tested in level 2. No new tactors were activated in this condition, only the vibrotactile patterns changed.

\section{Dependent Variables}

The effects of increasing the number of haptic seat alerts were investigated using measures of driver response time, accuracy, and subjective ratings. These measures are described below.

\section{Manual Response Accuracy}

Participants were instructed to perform a specific driving maneuver in response to each alert. Drivers had to press the brake pedal in response to the FCW, CSW, and IVW alerts. This driving maneuver was assigned to these alerts because they were intended to warn drivers to slow down. Drivers had to steer right in response to the left LCW and LDW alerts. This driving maneuver was assigned to these alerts because they were intended to warn drivers to steer away from a crash threat. Similarly, drivers had to steer left in response to right LCW and LDW alerts.

The manual response taken by participants was observed and scored. However, there was a concern regarding whether drivers' should be penalized for making partial maneuvers prior to executing the correct manual response. For example, a driver that 
jerks the steering wheel to the left prior to steering the vehicle into the right lane after receiving a left LCW alert. Here, jerking the steering wheel into the crash threat is an indication of inefficient information processing, but it is not practically concerning since the action is not severe enough to affect the trajectory of the vehicle. For this reason, two methods of scoring the data were used. For the first method, which was termed practical accuracy, the task was performed correctly when the participant executed the proper manual response to the haptic seat alert at some point after the alert. Table 3 lists the manual responses that are required and permitted for each alert in order for a trial to be scored as correct. The premise behind the practical manual response measure is that if the correct driving maneuver is performed, then the crash threat would be avoided.

Table 3. Practical Manual Response Accuracy

\begin{tabular}{|c|c|c|c|c|c|c|c|}
\hline $\begin{array}{c}\text { Manual } \\
\text { Response }\end{array}$ & FCW & CSW & IVW & LDW L & LDW R & LCW L & LCW R \\
\hline $\begin{array}{c}\text { Foot Off } \\
\text { Throttle }\end{array}$ & Allowed & Allowed & Allowed & Allowed & Allowed & Allowed & Allowed \\
\hline $\begin{array}{c}\text { Press } \\
\text { Brake } \\
\text { Pedal }\end{array}$ & Required & Required & Required & Allowed & Allowed & Allowed & Allowed \\
\hline $\begin{array}{c}\text { Steer } \\
\text { Left }\end{array}$ & Allowed & Allowed & Allowed & Allowed & Required & Allowed & Required \\
\hline $\begin{array}{c}\text { Steer } \\
\text { Right }\end{array}$ & Allowed & Allowed & Allowed & Required & Allowed & Required & Allowed \\
\hline $\begin{array}{c}\text { Jerk } \\
\text { Left }\end{array}$ & Allowed & Allowed & Allowed & Allowed & Allowed & Allowed & Allowed \\
\hline $\begin{array}{c}\text { Jerk } \\
\text { Right }\end{array}$ & Allowed & Allowed & Allowed & Allowed & Allowed & Allowed & Allowed \\
\hline
\end{tabular}

At the same time, there was an interest in measuring drivers' ability to efficiently process the information conveyed by the haptic seat alerts. A second method, which was termed drivers' theoretical accuracy, scored drivers' responses as correct when they only executed the proper manual response to the haptic seat alert. Here, performing a response other than the required maneuver was considered incorrect. It should be noted that this measure did allow drivers to press the brake pedal when responding to any of the LCW or LDW alerts. This was done because drivers typically pressed the brake pedal to control the lane changes they made in response to the LCW and LDW alerts. Table 4 presents the manual responses that were required and allowed for a trial to be scored as correct. 
Table 4. Theoretical Manual Response Accuracy

\begin{tabular}{|c|c|c|c|c|c|c|c|}
\hline $\begin{array}{l}\text { Manual } \\
\text { Response }\end{array}$ & FCW & CSW & IVW & LDW L & LDW R & LCW L & LCW R \\
\hline $\begin{array}{l}\text { Foot Off } \\
\text { Throttle }\end{array}$ & Allowed & Allowed & Allowed & Allowed & Allowed & Allowed & Allowed \\
\hline $\begin{array}{l}\text { Press } \\
\text { Brake } \\
\text { Pedal }\end{array}$ & Required & Required & Required & Allowed & Allowed & Allowed & Allowed \\
\hline $\begin{array}{c}\text { Steer } \\
\text { Left }\end{array}$ & $\begin{array}{c}\text { Not } \\
\text { Allowed }\end{array}$ & $\begin{array}{c}\text { Not } \\
\text { Allowed }\end{array}$ & $\begin{array}{c}\text { Not } \\
\text { Allowed }\end{array}$ & $\begin{array}{c}\text { Not } \\
\text { Allowed }\end{array}$ & Required & $\begin{array}{c}\text { Not } \\
\text { Allowed }\end{array}$ & Required \\
\hline $\begin{array}{l}\text { Steer } \\
\text { Right }\end{array}$ & $\begin{array}{c}\text { Not } \\
\text { Allowed }\end{array}$ & $\begin{array}{c}\text { Not } \\
\text { Allowed }\end{array}$ & $\begin{array}{c}\text { Not } \\
\text { Allowed }\end{array}$ & Required & $\begin{array}{c}\text { Not } \\
\text { Allowed }\end{array}$ & Required & $\begin{array}{c}\text { Not } \\
\text { Allowed }\end{array}$ \\
\hline $\begin{array}{l}\text { Jerk } \\
\text { Left }\end{array}$ & $\begin{array}{c}\text { Not } \\
\text { Allowed }\end{array}$ & $\begin{array}{c}\text { Not } \\
\text { Allowed }\end{array}$ & $\begin{array}{c}\text { Not } \\
\text { Allowed }\end{array}$ & $\begin{array}{c}\text { Not } \\
\text { Allowed }\end{array}$ & $\begin{array}{c}\text { Not } \\
\text { Allowed }\end{array}$ & $\begin{array}{c}\text { Not } \\
\text { Allowed }\end{array}$ & $\begin{array}{c}\text { Not } \\
\text { Allowed }\end{array}$ \\
\hline $\begin{array}{c}\text { Jerk } \\
\text { Right }\end{array}$ & $\begin{array}{c}\text { Not } \\
\text { Allowed }\end{array}$ & $\begin{array}{c}\text { Not } \\
\text { Allowed }\end{array}$ & $\begin{array}{c}\text { Not } \\
\text { Allowed }\end{array}$ & $\begin{array}{c}\text { Not } \\
\text { Allowed }\end{array}$ & $\begin{array}{c}\text { Not } \\
\text { Allowed }\end{array}$ & $\begin{array}{c}\text { Not } \\
\text { Allowed }\end{array}$ & $\begin{array}{c}\text { Not } \\
\text { Allowed }\end{array}$ \\
\hline
\end{tabular}

\section{Manual Response Time}

The elapsed time from the alert being generated to the initiation of a driving maneuver was recorded as drivers' response time. The exact point the alert was generated was recorded by the DAS. The video footage was then advanced frame-by-frame until the driving behavior was observed to begin. The synchronization point of the alert and the beginning of the driving behavior were recorded. The elapsed time between these two points was later computed in SAS, a statistical software package. Since the video footage was recorded at $29.97 \mathrm{~Hz}$, a measure of drivers' response time could be made with an error of $\pm 0.067 \mathrm{~s}$.

\section{Verbal Response Accuracy}

Participants were asked to verbally indicate the type of alert that was presented after they performed a driving maneuver. Their performance was scored based on their final verbal response to each alert. Table 5 lists the verbal responses that were assigned to the seven alerts. It should be noted that participants' verbal responses were scored on meaning, and not exact terminology. For example, "Car Ahead" and "Stopping Car" were both acceptable verbal responses to the FCW alert.

Table 5. Verbal Responses Assigned to Alerts 


\begin{tabular}{|c|c|}
\hline CAS Alert & Verbal Response \\
\hline FCW & "Car Ahead" \\
\hline CSW & "Sharp Turn" \\
\hline IVW & "Red Light" \\
\hline Right LCW & "Car Right" \\
\hline Left LCW & "Car Left" \\
\hline Right LDW & "Drift Right" \\
\hline Left LDW & "Drift Left" \\
\hline
\end{tabular}

\section{Driver Ratings}

Participants' perception of the task's difficulty was measured at each level of the number of alerts independent variable. Appendices $\mathrm{E}$ and $\mathrm{F}$ present the questionnaires used for this experiment.

\section{Hypotheses}

In presenting the first research question, Hick (1952) and Hyman's (1953), as well as Miller's (1956), applications of Information Theory to explain human performance in selecting a response to multiple stimuli were discussed. Information Theory was used to predict that people's ability to correctly, and quickly, select an alternative would degrade as the number of alternatives available increased. Applying this theory to the design of a haptic driver seat, one would expect drivers' manual response time to increase, and their manual response accuracy to degrade, as the number of alerts increased. However, before hypothesizing the existence of this effect, it is important to realize that the haptic seat was designed to be intuitive by incorporating location compatibility into its design. That is, the vibrations in the seat were spatially congruent with the areas of danger the seat was alerting drivers too. The alerts that warned drivers to press the brake pedal to avoid forward crash threats were presented through tactors along the front edge of the seat pan. The alerts that warned drivers to steer away from left crash threats were presented through the back-left corner of the seat pan. Likewise, the alerts that warned drivers to steer away from right crash threats were presented through the back-right corner of the seat pan. Since this stimulus-response mapping was true for all alerts, regardless of the exact meaning they communicated, the results were expected to reveal a departure from information theory. Thus, drivers' manual response times and accuracy were hypothesized to not increase as the number of alerts increased. However, participants' verbal response accuracy was hypothesized to decrease as the number of alerts incremented. This was because drivers had to memorize what each of the seven alerts meant to successfully perform this task. Participants' perception of task difficulty was hypothesized to increase as the number of alerts increased. Furthermore, based on earlier research by Fitch et al. (2005), it was hypothesized that older drivers would err more, as well as take longer to manually respond, than younger drivers. No gender 
differences were hypothesized.

\section{RESULTS}

\section{Manual Response Accuracy}

This section presents the manual response accuracy results in terms of both practical accuracy and theoretical accuracy.

\section{Practical Manual Response Accuracy}

In general, drivers performed the correct maneuver in response to the haptic seat alerts with adequate accuracy (above 94 percent correct for each participant grouping. The worst score produced on a per driver basis was 86 percent correct, which was produced by an older male driver in the seven alerts condition). Drivers made the correct practical manual response 100 percent of the time when one alert was presented in a condition. When three alerts were presented, they made the correct response 99 percent of the time. When seven alerts were presented, they made the correct response 97 percent of the time. A Cochran-Mantel-Haenszel (CMH) test (an appropriate nonparametric test) revealed that these slight differences were statistically significant $(\mathrm{Q}(2)=8.9264, p=0.0115)$.

Figure 16 shows driver's practical manual response accuracy broken down by the age and gender of the drivers. It can be seen that the manual response performance exhibited by younger drivers did not degrade as the number of alerts increased from one to seven. 


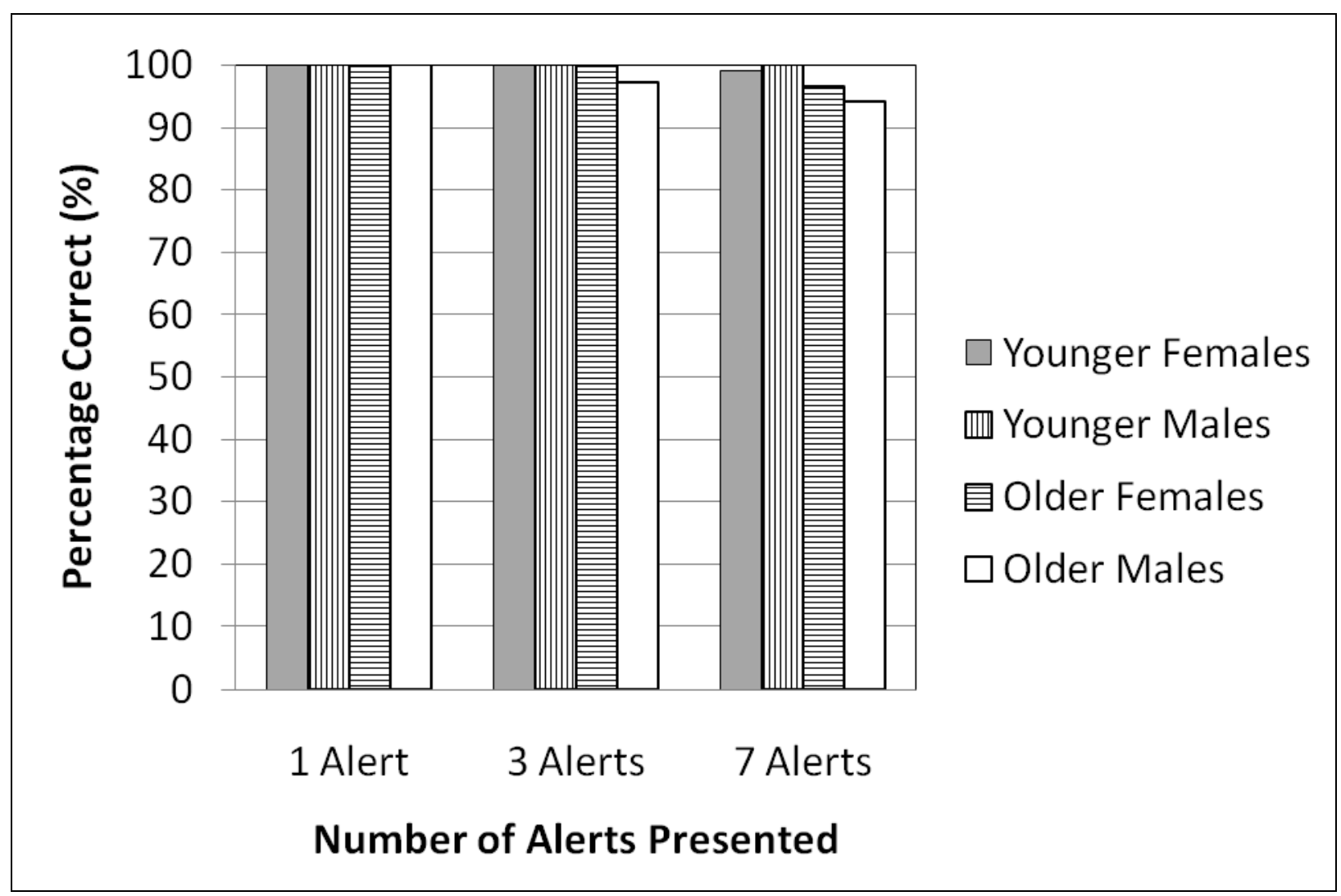

Figure 16. Drivers' practical manual response accuracy broken down by the age and gender of the drivers.

A CMH test could not be used to investigate how younger males' accuracy varied by the number of alerts since they obtained $100 \%$ accuracy in each set. A CMH test did not find younger females', older males', or older females' accuracy to significantly vary as the number of alerts increased $(\mathrm{Q}(2)=1.1458, p=0.5639, \mathrm{Q}(2)=3.6325, p=0.1626$, and $\mathrm{Q}(2)=5.2492, p=0.0725$, respectively).

Overall, there was a significant Age main effect $\left(\chi^{2}(1)=21.9537, p<0.0001\right.$. Fisher's exact $p<0.0001)$. Older drivers accurately identified 97 percent of the alerts, while younger drivers accurately identified 100 percent of the alerts. When just considering the responses made when three alerts were presented in a condition, there was no significant difference in accuracy between younger and older drivers $\left(\chi^{2}(1)=3.0210, p=0.0822\right.$. Fisher's exact $p=0.2483$ ). However, when just considering the responses made when seven alerts were presented in a condition, there was significant difference in accuracy between younger and older drivers $\left(\chi^{2}(1)=19.1083, p<0.0001\right.$. Fisher's exact $p<$ $0.0001)$. Younger drivers were correct 100 percent of the time when seven alerts were presented in a condition, while older drivers were correct 95 percent of the time.

A significant difference in performance between males and females was not found $\left(\chi^{2}(1)\right.$ $=1.7212, p=0.1895$. Fisher's exact $p=0.2605)$. Females accurately identified 99 percent of the alerts, while males accurately identified 98 percent of the alerts. 


\section{Theoretical Manual Response Accuracy}

Because the theoretical manual response measure is more stringent than the practical manual response measure, drivers' theoretical manual response performance was found to degrade more as the number of alerts was increased from one to seven. Drivers correctly responded to all of the alerts when one alert was presented in a condition. They correctly identified 94 percent of the alerts when three alerts were presented in a condition. They correctly identified 95 percent of the alerts when seven alerts were presented in a condition. The number of alerts was found to be a significant main effect $(\mathrm{Q}(2)=8.4186, p=0.0149)$. Figure 17 shows driver's theoretical manual response accuracy broken down by the age and gender of the drivers.

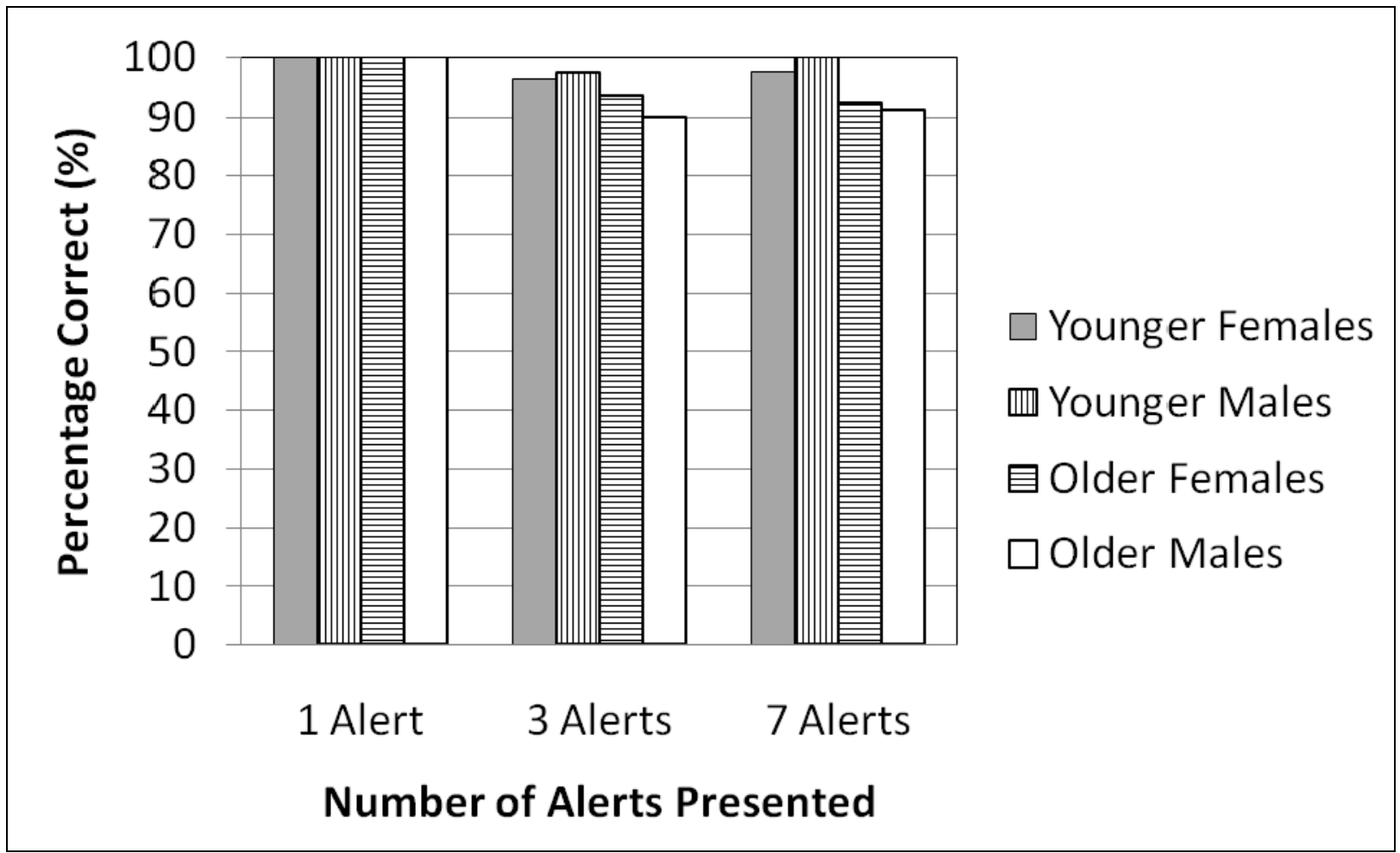

Figure 17. Drivers' theoretical manual response accuracy broken down by the age and gender of the drivers.

A significant Age effect was found $\left(\chi^{2}(1)=30.7884, p<0.0001\right.$. Fisher's exact $p<$ $0.0001)$. Younger drivers made the correct driving maneuver 98 percent of the time, while older drivers made the correct driving maneuver 93 percent of the time. When just considering drivers' performance when three alerts were presented in a condition, a significant Age effect was found $\left(\chi^{2}(1)=5.1373, p=0.0234\right.$. Fisher's exact $\left.p=0.0374\right)$. Older drivers correctly identified 92 percent of the alerts, while younger drivers correctly identified 97 percent of the alerts. When just considering drivers' performance when seven alerts were presented in a condition, a significant Age effect was also found $\left(\chi^{2}(1)\right.$ $=27.3385, p<0.0001$. Fisher's exact $p<0.0001)$. Older drivers correctly identified 92 percent of the alerts, while younger drivers correctly identified 99 percent of the alerts. 
Both males and females correctly identified 95 percent of the alerts.

\section{Manual Response Time}

Response time outliers were removed from the data prior to performing response time analyses. Appendix G documents which outliers were removed. The results in this section are presented in terms of both practical and theoretical response times.

\section{Practical Manual Response Time}

All response times observed when drivers performed the correct practical manual response are analyzed in this section. Figure 18 shows the histogram of drivers' practical manual response times to one, three, and seven alerts. 


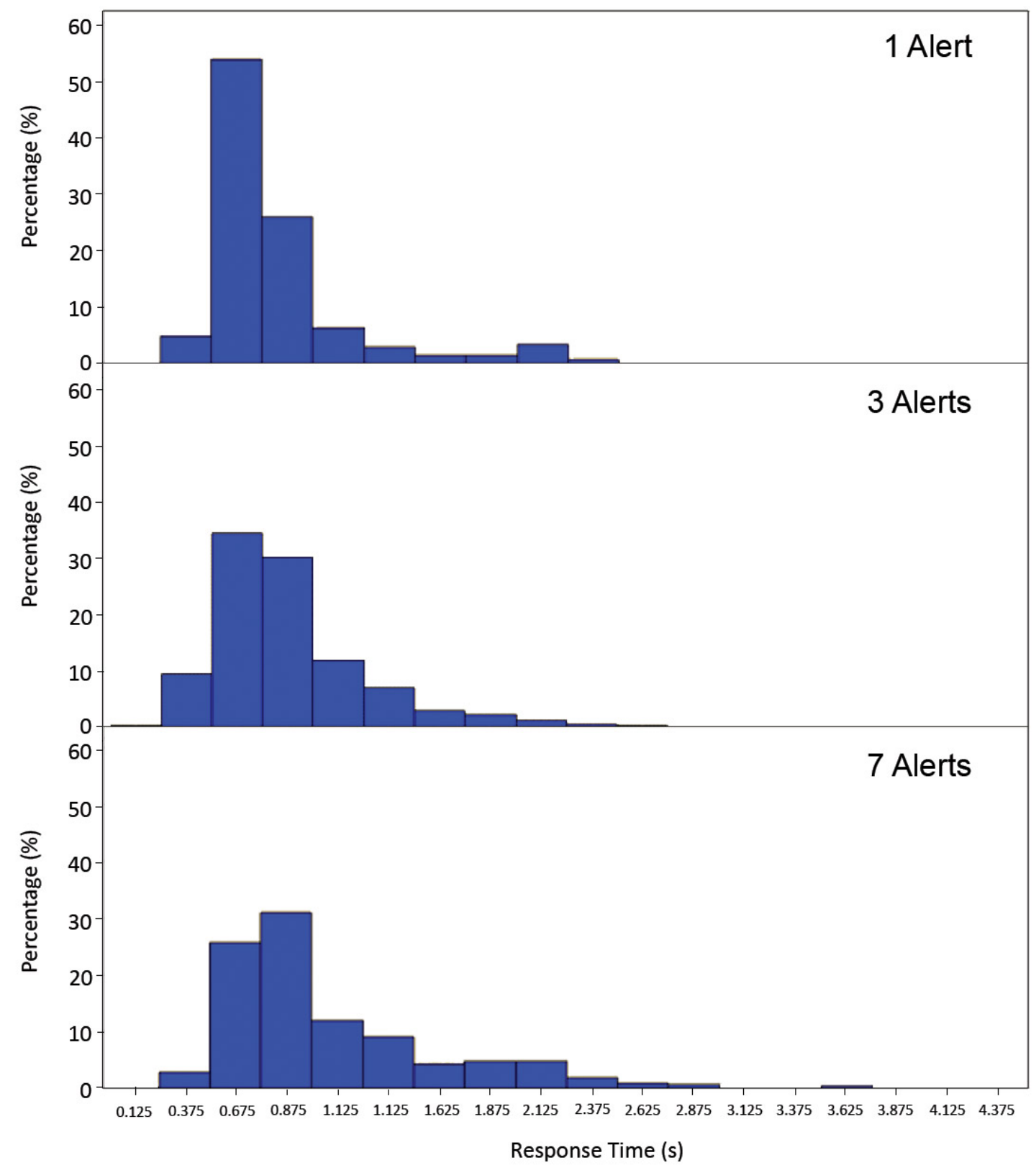

Figure 18. Histogram of drivers' practical manual response times to one, three, and seven alerts.

A 2 (Age) x 2 (Gender) x 3 (\#Alerts) mixed factors ANOVA was performed on the practical manual response time dependent variable. The number of alerts was found to be a significant main effect $(\mathrm{F}(2,40)=10.74, p=0.0002)$. Drivers' mean response time to just the FCW alert was $0.831 \mathrm{~s}($ s.e. $=0.031 \mathrm{~s})$. Drivers' mean response time when three alerts were presented in a condition was $0.847 \mathrm{~s}(\mathrm{~s} . \mathrm{e} .=0.017 \mathrm{~s})$. Drivers' mean response 
time when seven alerts were presented in a condition was $1.087 \mathrm{~s}(\mathrm{~s} . \mathrm{e} .=0.019 \mathrm{~s})$. A Tukey-Kramer multiple comparisons test revealed that the drivers' mean response time to seven alerts was significantly different from their mean response time to one alert ( $p=$ $0.0175)$, as well as three alerts $(p=0.0005)$.

Figure 19 shows drivers' mean practical manual response time to one, three, and seven alerts broken down by their age and gender. A significant Age $\mathrm{x}$ \#Alerts interaction was found $(\mathrm{F}(2,40)=4.55, p=0.0166)$. It can be seen that the younger drivers' response times did not increase as much as older drivers when the number of alerts increased from one to seven. A Tukey-Kramer multiple comparisons test revealed that the older drivers' mean response time to seven alerts was significantly different from their mean response time to one alert $(p=0.0175)$, as well as three alerts $(p=0.0004)$. Older drivers' mean response time to seven alerts was significantly different from younger drivers' mean response time to one alert $(p=0.0017))$, three alerts $(p<0.0001)$ and seven alerts $(p<$ $0.0001)$.

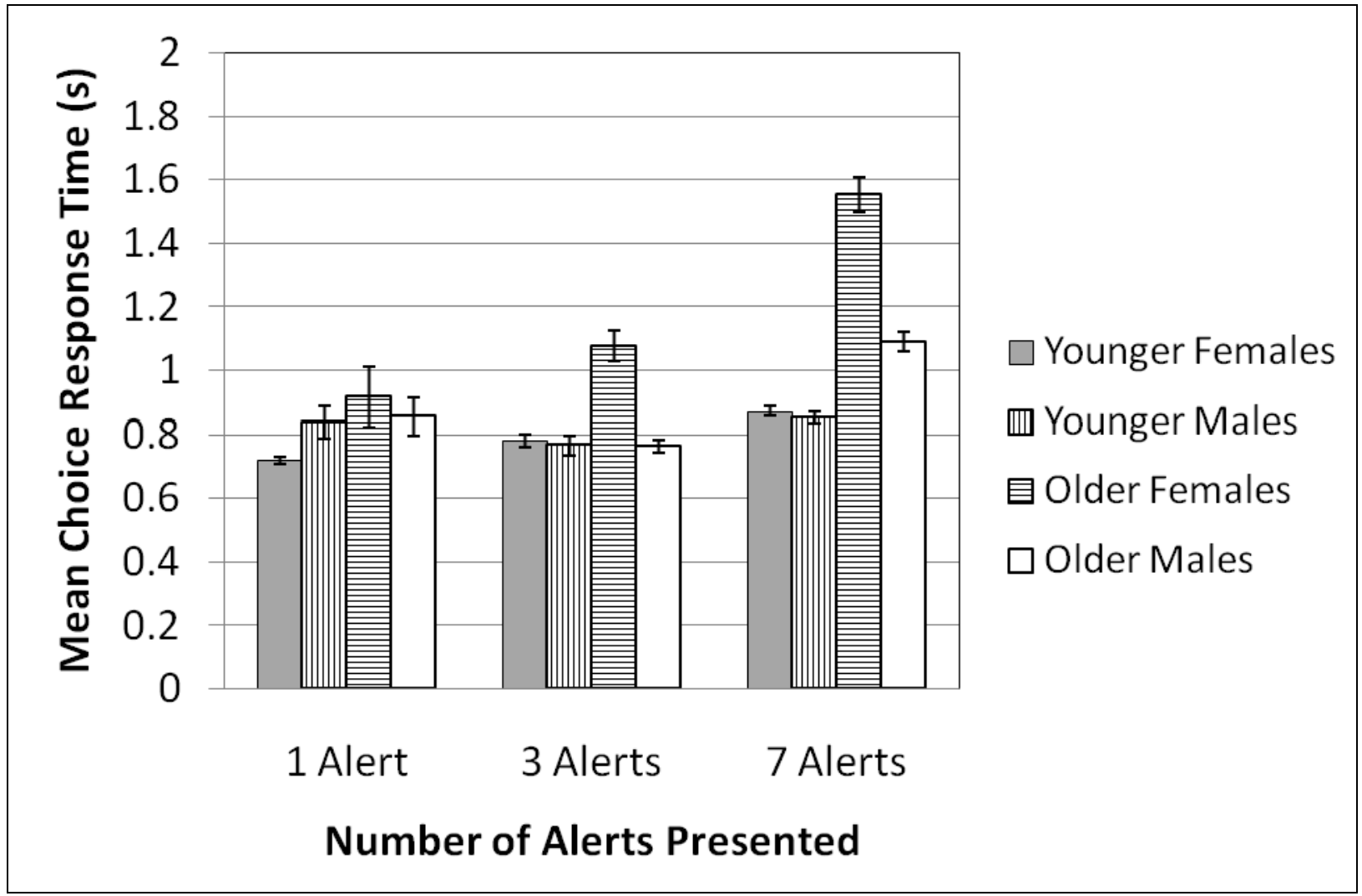

Figure 19. Alert practical manual response time.

The drivers' age was found to be marginally significant effect $(\mathrm{F}(2,20)=4.37, p=$ 0.0484 ). Younger drivers' mean response time was $0.830 \mathrm{~s}$ (s.e. $=0.009 \mathrm{~s}$ ), while older drivers' mean response time was $1.163 \mathrm{~s}(\mathrm{~s} . \mathrm{e} .=0.023 \mathrm{~s})$.

The quantiles for driver's manual response time to one, three, and seven alerts are shown 
in Table 6. The extremely short minimum response time in the 7-Alert condition was verified and is associated with a steering input. Figure 20 presents drivers' $90^{\text {th }}$ percentile response times according to their age and gender.

Table 6. Drivers' Practical Response Time Quantiles by Number of Alerts

\begin{tabular}{|c|c|c|c|}
\hline Quantile & 1 Alert & 3 Alerts & 7 Alerts \\
\hline $100 \%$ Max & 2.300 & 2.600 & 6.400 \\
\hline $99 \%$ & 2.133 & 2.067 & 3.467 \\
\hline $95 \%$ & 2.000 & 1.500 & 2.233 \\
\hline $90 \%$ & 1.200 & 1.333 & 1.933 \\
\hline $75 \%$ Q3 & 0.867 & 0.967 & 1.267 \\
\hline $50 \%$ Median & 0.733 & 0.767 & 0.900 \\
\hline $25 \%$ Q1 & 0.633 & 0.600 & 0.700 \\
\hline $10 \%$ & 0.567 & 0.500 & 0.600 \\
\hline $5 \%$ & 0.533 & 0.467 & 0.533 \\
\hline $1 \%$ & 0.333 & 0.333 & 0.433 \\
\hline $0 \%$ Min & 0.333 & 0.167 & 0.067 \\
\hline
\end{tabular}

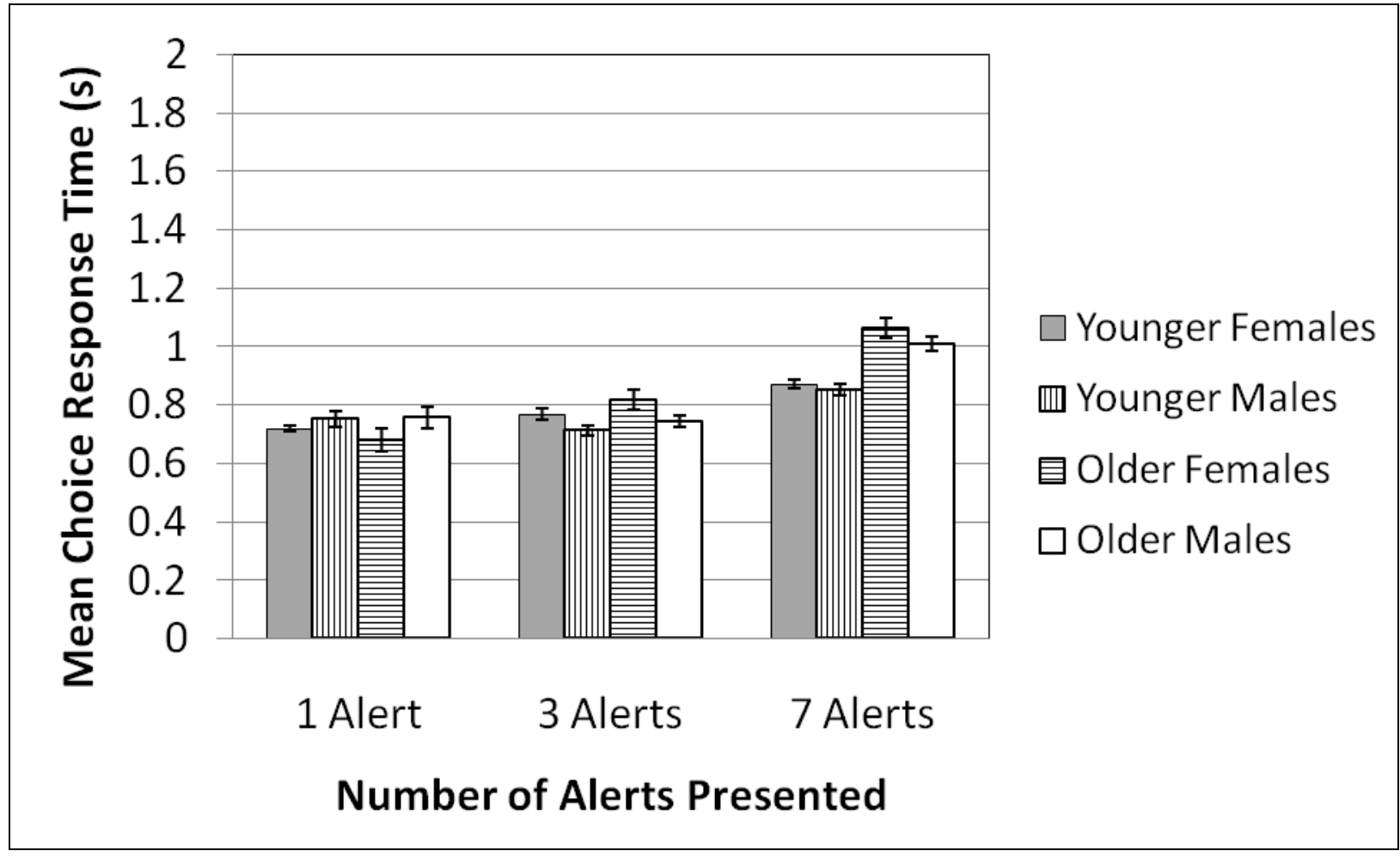

Figure 20. Drivers' $90^{\text {th }}$ percentile practical manual response times. 


\section{Hick-Hyman Law of Choice Response Time}

The amount of information conveyed by an alert was quantified according to the probability that it was generated in a given condition. The amount of information conveyed by an alert, termed $\mathrm{H}_{\mathrm{s}}$, was assessed as:

$$
\mathrm{H}_{\mathrm{s}}=\log _{2}\left(\frac{1}{\mathrm{P}_{\mathrm{i}}}\right)
$$

where $\mathrm{P}_{\mathrm{i}}$ is the probability that a specific type of alert, such as an FCW alert, was generated. Since an FCW alert was possible in each of the alert conditions, the amount of information it conveyed in each condition can be compared. In the first experimental condition, the probability that an FCW alert was generated was 1.0 since it was the only alert generated. The information conveyed by this alert was therefore 0 bits (Table 7). In the second experimental condition, the probability that an FCW alert was generated was 0.333 . The information conveyed by it was therefore 1.585 bits. In the third experimental condition, the probability that an FCW alert was generated was 0.143 . The information conveyed by it was 2.807 bits. Figure 21 plots drivers' mean choice response time against the information conveyed by an FCW alert for a given experimental condition. As predicted by the Hick-Hyman law of choice response time, drivers' mean choice response times appear to increase linearly with increased information $\left(\mathrm{R}^{2}=0.9031\right)$. The slope of the function, $\mathrm{b}=0.133 \mathrm{~s}$, represents the amount of additional processing time that is required to process each added bit of information. The intercept, $\mathrm{a}=0.801 \mathrm{~s}$, represents the sum of cognitive processes that are unrelated to the reduction of uncertainty, such as the time taken to perceive a haptic alert and execute a manual response. A caveat here, however, is that the vibration alerts were intuitively mapped to the desired manual response. Alerts presented without this mapping may require even more attentional resources, and therefore processing time, by drivers. The slope of the line would be expected to be steeper in this case. It should also be noted that only three data points were used in this analysis, and so the results should be considered explorative rather than definitive.

Table 7. Computation of Information Conveyed by an FCW Alert

\begin{tabular}{|l|c|c|c|c|}
\hline \multicolumn{1}{|c|}{ Event $\mathbf{i}$} & $\mathbf{P}_{\mathbf{i}}$ & $\frac{\mathbf{1}}{\mathbf{P}_{\mathbf{i}}}$ & $\mathbf{H}_{\mathbf{s}}$ (bits) & $\begin{array}{c}\text { Mean Manual } \\
\text { Response Time (s) }\end{array}$ \\
\hline FCW Only & 1.000 & 1 & 0.000 & 0.831 \\
\hline FCW with Two Alerts & 0.333 & 3 & 1.585 & 0.941 \\
\hline FCW with Six Alerts & 0.143 & 7 & 2.807 & 1.215 \\
\hline
\end{tabular}




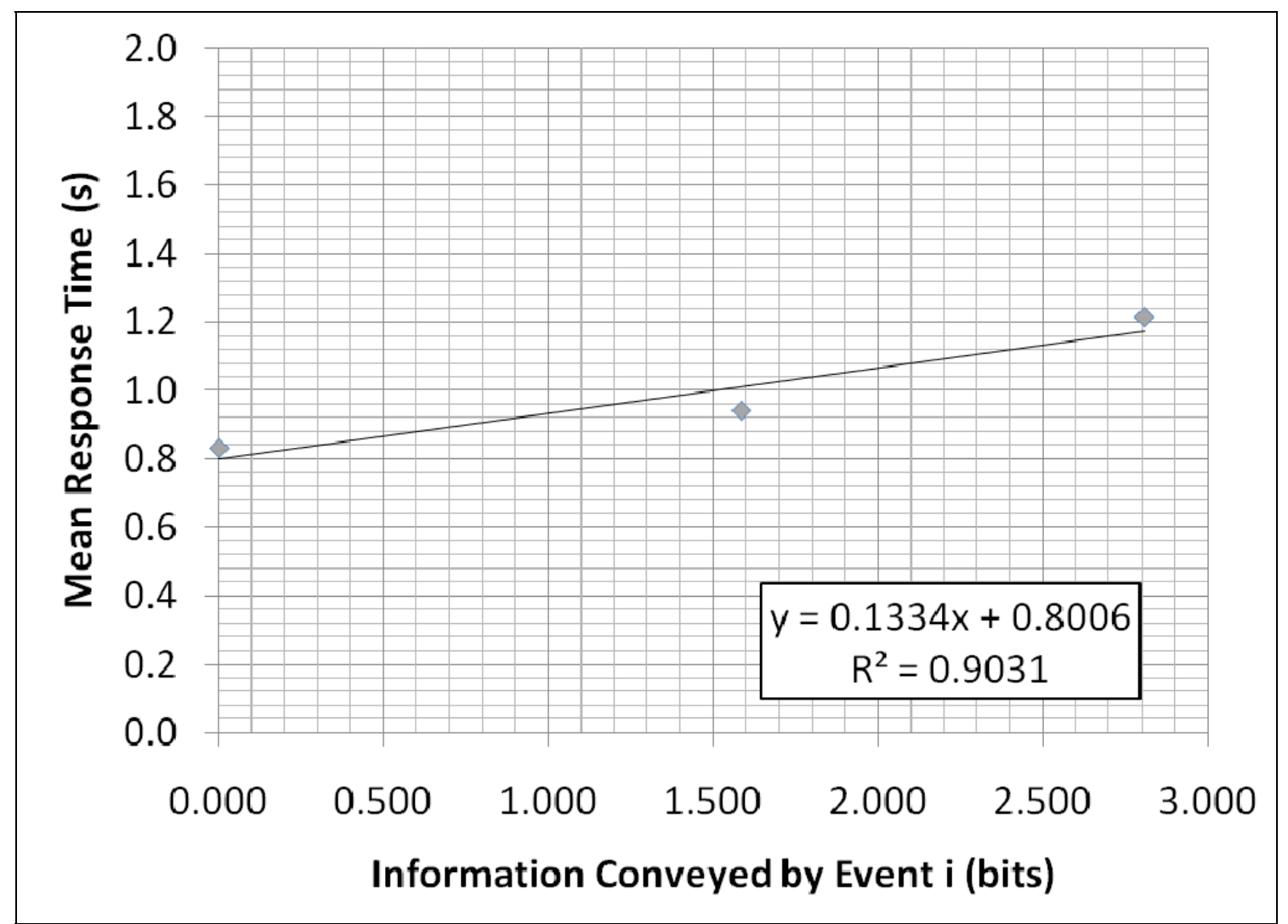

Figure 21. The Hick-Hyman Law of choice response time as exemplified by drivers' mean response times to an FCW alert presented on its own, with two other alerts, and with six other alerts.

Figure 22 presents drivers' mean brake response times to the FCW alert broken down by drivers' age and gender. Closer inspection reveals that the steady increase in brake response times to $\mathrm{FCW}$ alerts was greater for older drivers relative to younger drivers. 


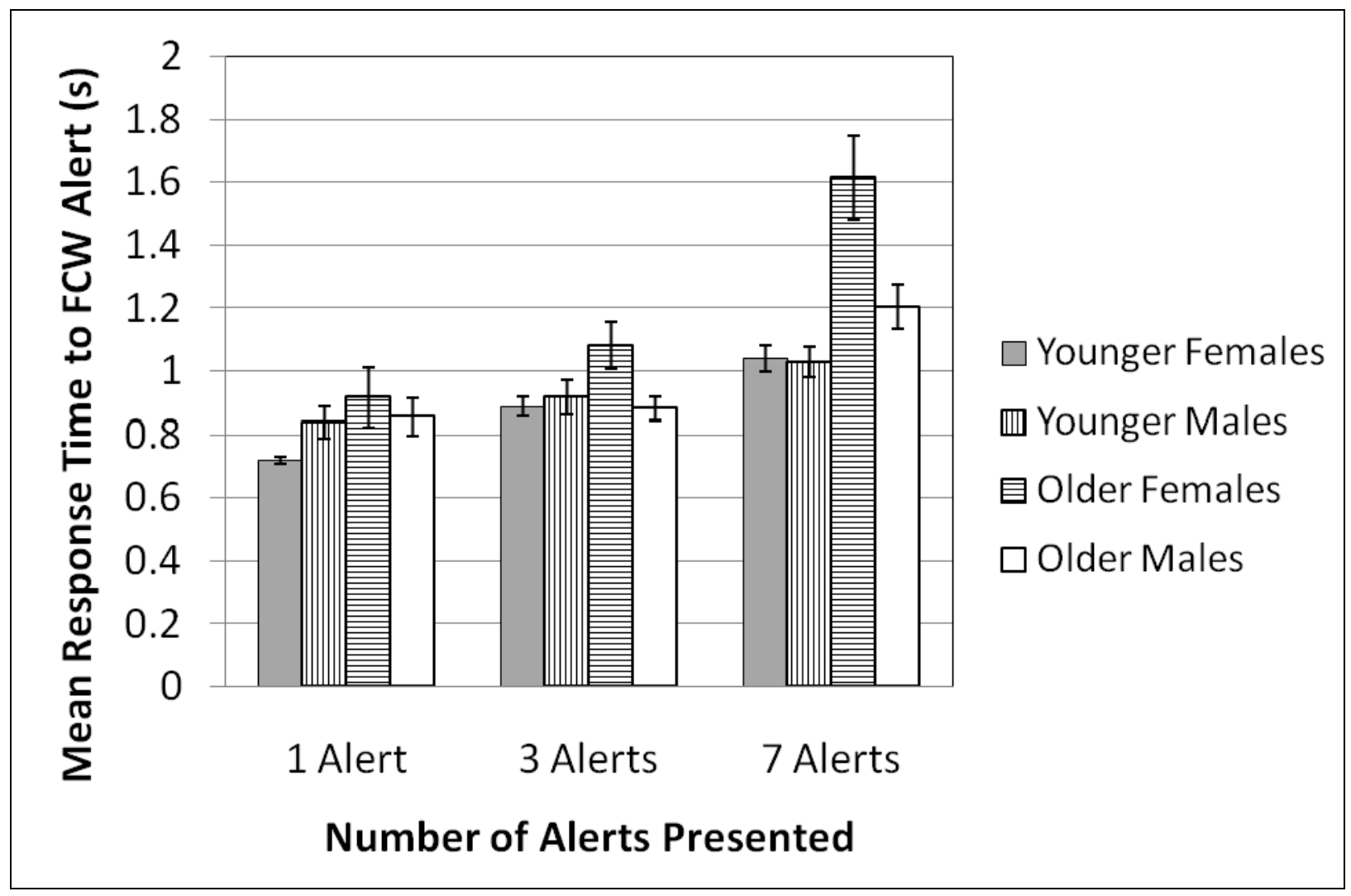

Figure 22. Drivers' practical manual response time to FCW alerts.

Examination of drivers' manual response times to both left and right LCW alerts also revealed latencies as the number of alerts increased. Drivers' mean response time to a left LCW was $0.787 \mathrm{~s}$ (s.e. $=0.030 \mathrm{~s}$ ) when it was presented with FCW and right LCW alerts, while it increased to $1.082 \mathrm{~s}($ s.e. $=0.057 \mathrm{~s})$ when it was presented with six alerts in a condition. Similarly, drivers' mean response time to a right LCW was $0.810 \mathrm{~s}$ (s.e. $=$ $0.031 \mathrm{~s}$ ) when it was presented with FCW and left LCW alerts, while it increased to $0.988 \mathrm{~s}$ (s.e. $=0.050 \mathrm{~s}$ ) when it was presented with six alerts in a condition. It is important to keep in mind at this time that these alerts were presented to drivers without any collision context. It is foreseeable that drivers' response times may be quicker when primed by a pre-incident maneuver or unveiling crash threat.

\section{Theoretical Manual Response Time}

All response times observed when participants performed the correct theoretical manual response are analyzed in this section. Figure 23 shows the histogram of drivers' theoretical manual response times to one, three, and seven alerts. 


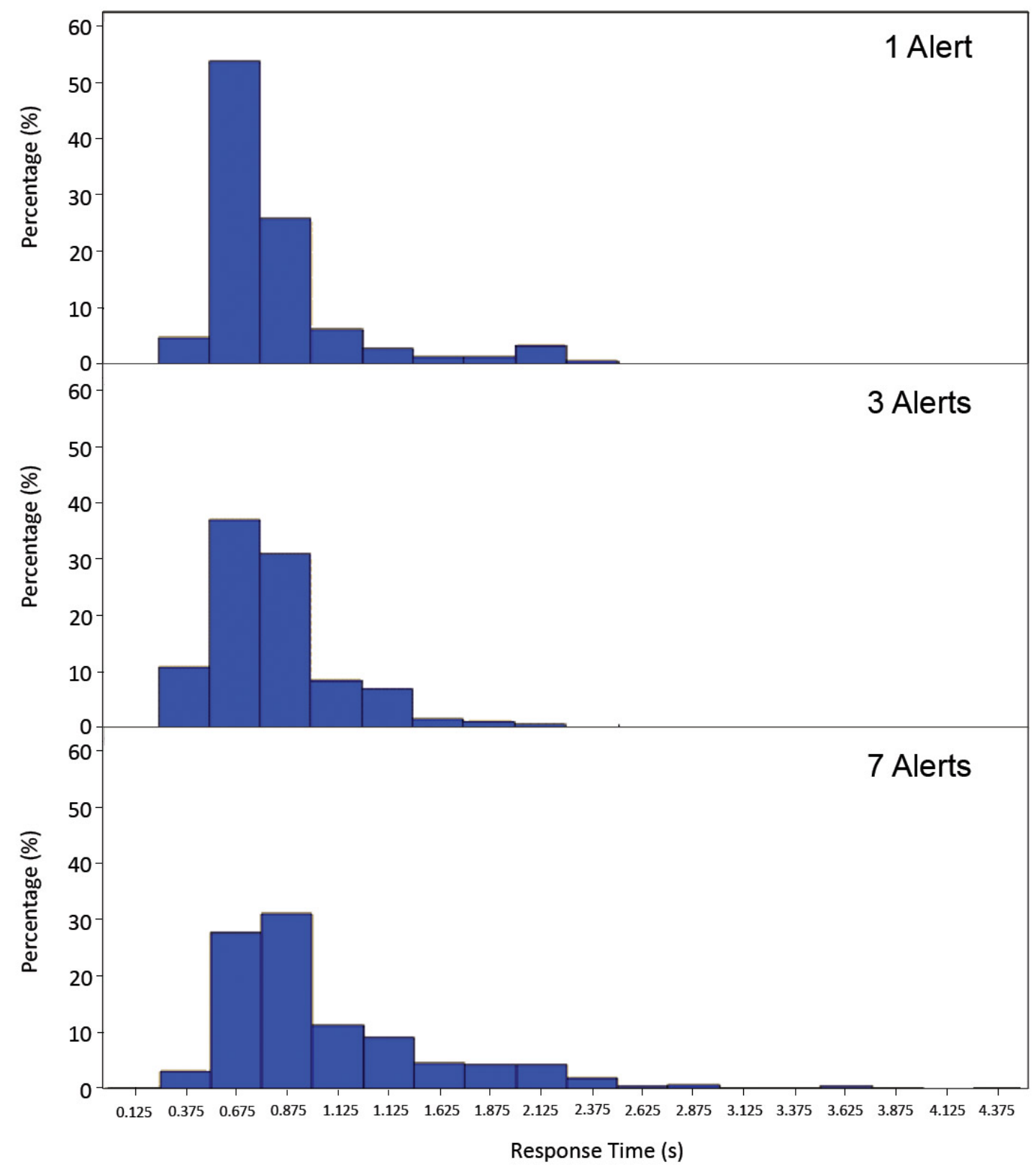

Figure 23. Histogram of drivers' theoretical manual response time to one, three, and seven alerts.

A 2 (Age) x 2 (Gender) x 3 (\#Alerts) mixed factors ANOVA was performed on the theoretical manual response time dependent variable. The number of alerts was found to be a significant main effect $(\mathrm{F}(2,40)=11.49, p=0.0001)$. Drivers' mean response time to just the FCW alert was $0.831 \mathrm{~s}($ s.e. $=0.031 \mathrm{~s})$. Their mean response time when three alerts were presented in a condition was $0.833 \mathrm{~s}$ (s.e. $=0.017 \mathrm{~s}$ ). Their mean response time when seven alerts were presented in a condition was $1.08 \mathrm{~s}(\mathrm{~s} . \mathrm{e} .=0.018 \mathrm{~s})$. These 
mean response times were slightly faster than drivers' practical manual response times. A Tukey-Kramer multiple comparisons test revealed that the drivers' mean response time to one alert was significantly different from their mean response time to seven alerts $(p=$ 0.0174 ). Drivers' mean response time to three alerts was also significantly different from their mean response time to seven alerts $(p=0.0003)$.

Figure 24 shows drivers' mean theoretical manual response times to one, three, and seven alerts broken down by their age and gender. A significant Age $\mathrm{x}$ \#Alerts interaction was found $(\mathrm{F}(2,40)=4.48, p=0.0176)$. It can be seen that the younger drivers' response times did not increase as much as older drivers did as the number of alerts increased from one to seven. A Tukey-Kramer multiple comparisons test revealed that the older drivers' mean response time to seven alerts was significantly different from their mean response time to one alert $(p=0.0015)$, as well as three alerts $(p=0.0003)$. Older drivers' mean response time to seven alerts was significantly different from younger drivers' mean response time to one alert $(p=0.0168)$, three alerts $(p<0.0001)$ and seven alerts $(p<$ $0.0001)$.

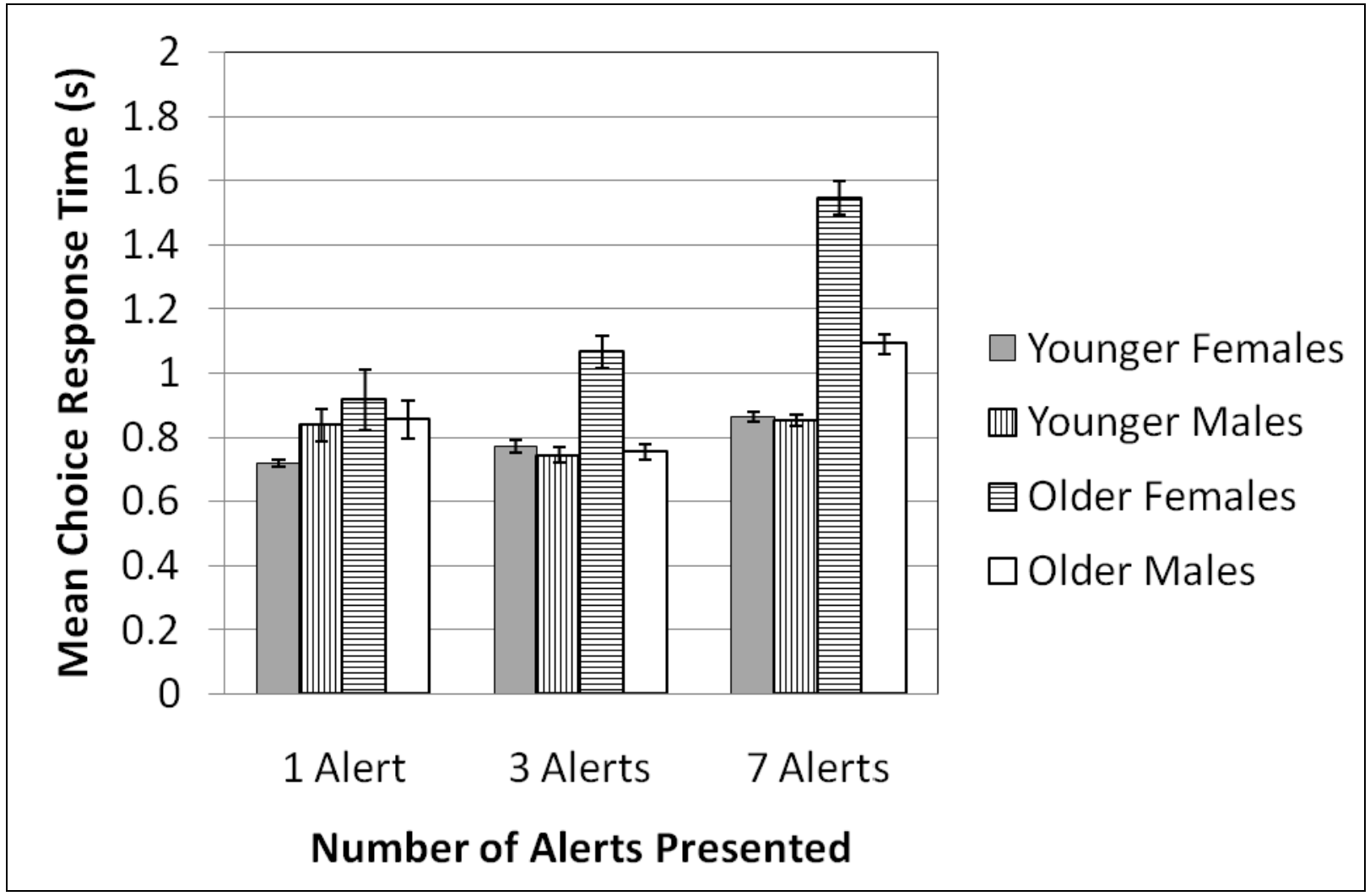

Figure 24. Drivers' theoretical manual response times by age and gender.

The drivers' age was found to be marginally significant $(\mathrm{F}(2,20)=4.37, p=0.0496)$. Younger drivers' mean response time was $0.823 \mathrm{~s}(\mathrm{~s} . \mathrm{e} .=0.009 \mathrm{~s}$ ), while older drivers' mean response time was $1.166 \mathrm{~s}(\mathrm{~s} . \mathrm{e}$. $=0.024 \mathrm{~s})$.

The quantiles for drivers' theoretical manual response time to one, three, and seven alerts 
are shown in Table 8 . Drivers' $90^{\text {th }}$ percentile theoretical manual response times broken down by age and gender are shown in Figure 25.

Table 8. Drivers' Theoretical Response Time Quantiles by Number of Alerts

\begin{tabular}{|c|c|c|c|}
\hline Quantile & 1 Alert & 3 Alerts & 7 Alerts \\
\hline $100 \%$ Max & 2.300 & 2.367 & 4.300 \\
\hline $99 \%$ & 2.133 & 2.033 & 3.367 \\
\hline $95 \%$ & 2.000 & 1.500 & 2.200 \\
\hline $90 \%$ & 1.200 & 1.300 & 1.917 \\
\hline $75 \%$ Q3 & 0.867 & 0.967 & 1.267 \\
\hline $50 \%$ Median & 0.733 & 0.767 & 0.900 \\
\hline $25 \%$ Q1 & 0.633 & 0.600 & 0.700 \\
\hline $10 \%$ & 0.567 & 0.500 & 0.600 \\
\hline $5 \%$ & 0.533 & 0.433 & 0.533 \\
\hline $1 \%$ & 0.333 & 0.333 & 0.433 \\
\hline $0 \%$ Min & 0.333 & 0.167 & 0.067 \\
\hline
\end{tabular}

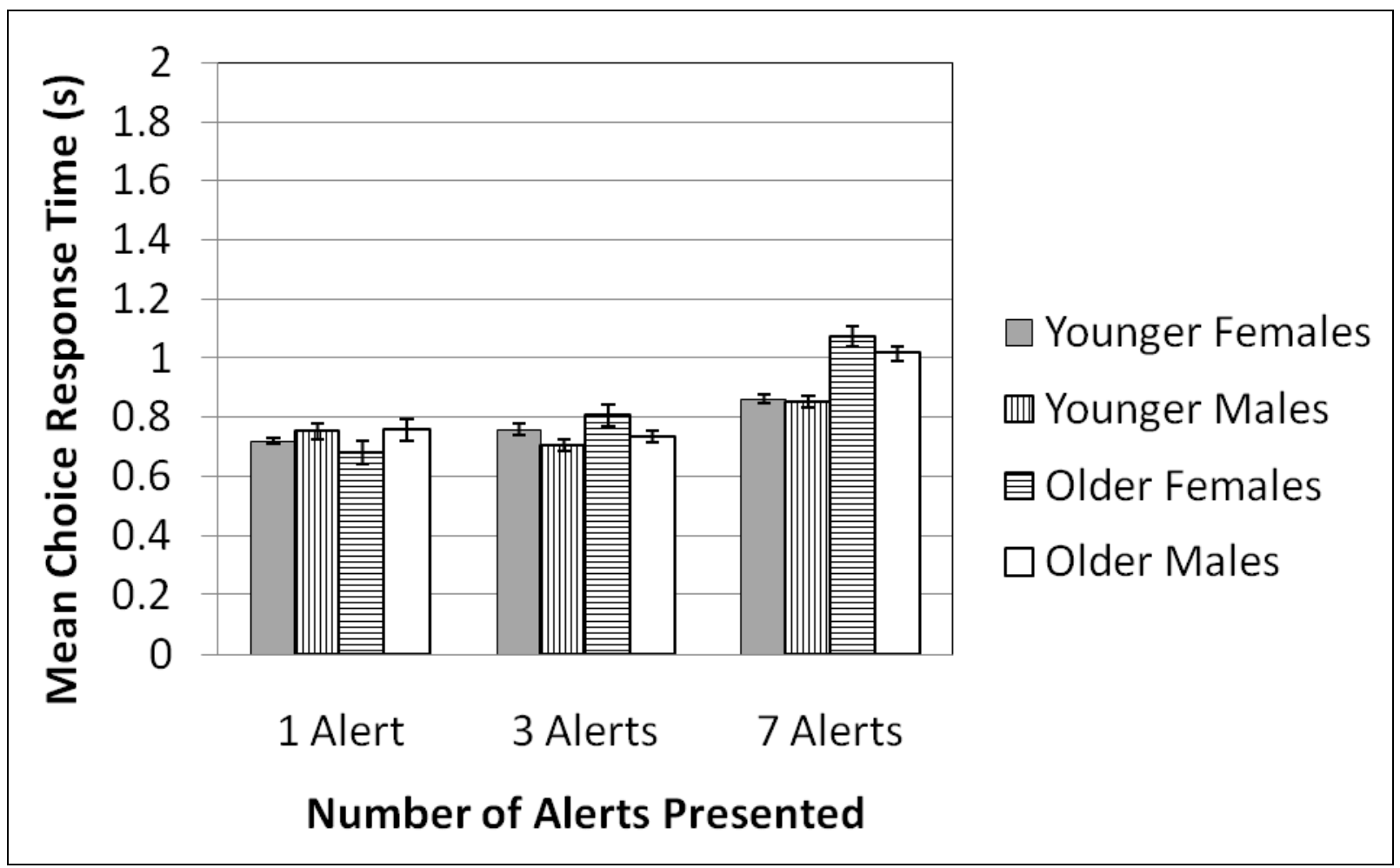

Figure 25. Drivers' $90^{\text {th }}$ percentile theoretical manual response times. 


\section{Speed Accuracy Tradeoff}

Response time and error rate represent two dimensions of the efficiency of processing information (Wickens \& Hollands, 1999). Drivers that correctly identify the alerts in a short amount of time are more efficient at procssing the alerts than drivers that take a longer time to identify the alerts and make mistakes. Figure 26 shows that drivers' performance generally moved from the top left of the graph (efficient information processing) down towards the bottom right of the graph (inefficient information processing) as the number of alerts increased from one to seven. This suggests that drivers had to use additional attentional resources to process the information contained in the alerts when the number of alerts was increased. It should be noted that movement from the top right of the graph down towards the bottom left of the graph does not represent a change in information processing efficiency, but rather represents drivers changing their disposition towards making a quick response versus an accurate response.

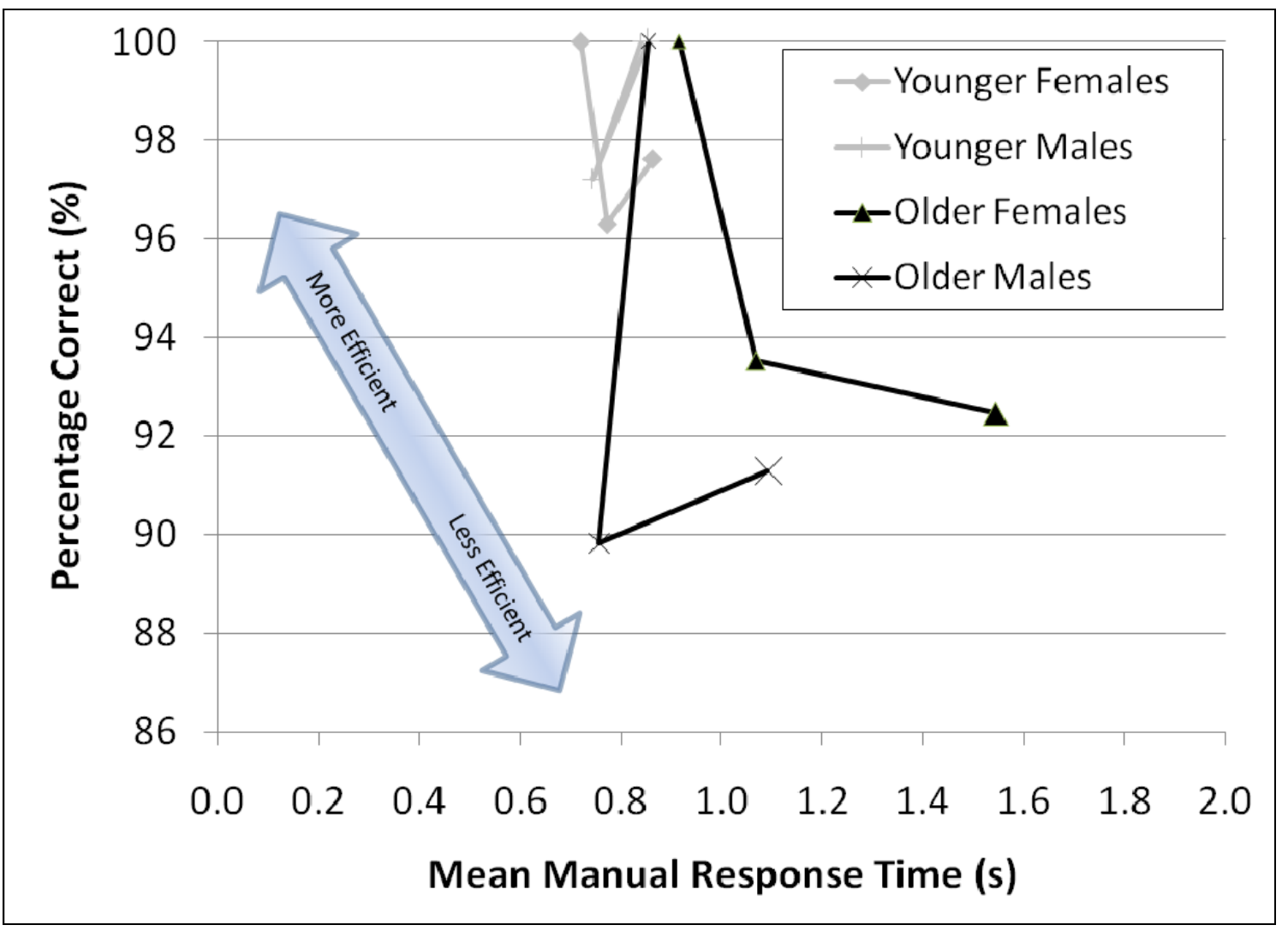

Figure 26. Speed-accuracy tradeoff investigation. For each data series, the smallest symbol represents the speed-accuracy data point associated with one alert, the next largest symbol represents the speed-accuracy data point associated with three alerts, and the largest symbol represents the speed-accuracy data point associated with seven alerts.

\section{Verbal Response Accuracy}

Drivers were generally accurate in verbally identifying the haptic seat alerts. Drivers 
accurately identified 100 percent of the alerts when one alert was presented in a condition, 99 percent of the alerts when three alerts were presented in a condition, and 87 percent when seven alerts were presented in a condition. A CMH test revealed that the number of alerts that could be generated had a significant effect on drivers' verbal response accuracy $(\mathrm{Q}(2)=67.457, p<0.0001)$.

There was a significant Age main effect $\left(\chi^{2}(1)=121.3536, p<0.0001\right.$. Fisher's exact $p$ $<0.0001$ ). Older drivers accurately identified 84 percent of the alerts, while younger drivers accurately identified 99 percent of the alerts. Figure 27 presents drivers' verbal response accuracy broken down by their age and gender. It can be seen that the performance of younger drivers did not degrade as the number of alerts presented in a condition increased. When just considering younger males, a CMH test did not find a significant difference in verbal response accuracy as the number of alerts increased $(\mathrm{Q}(2)$ $=0.6206, p=0.7332$ ). When just considering younger females, a CMH test did not find a significant difference in verbal response accuracy as the number of alerts increased $(\mathrm{Q}(2)=2.3124, p=0.3147)$. However, older drivers exhibited performance decrements as the number of alerts increased from three to seven. When just considering older males, a $\mathrm{CMH}$ test found a significant difference in verbal response accuracy as the number of alerts increased $(\mathrm{Q}(2)=34.1574, p<0.0001)$. Older male drivers correctly identified 100 percent of the alerts when just one was presented, 99 percent of the alerts when three were presented, and 78 percent of the alerts when seven alerts were presented. When just considering older females, a CMH test found a significant difference in verbal response accuracy as the number of alerts increased $(\mathrm{Q}(2)=38.6575, p<0001)$. Older female drivers correctly identified 100 percent of the alerts when just one was presented, 97 percent of the alerts when three were presented, and 73 percent of the alerts when seven alerts were presented. 


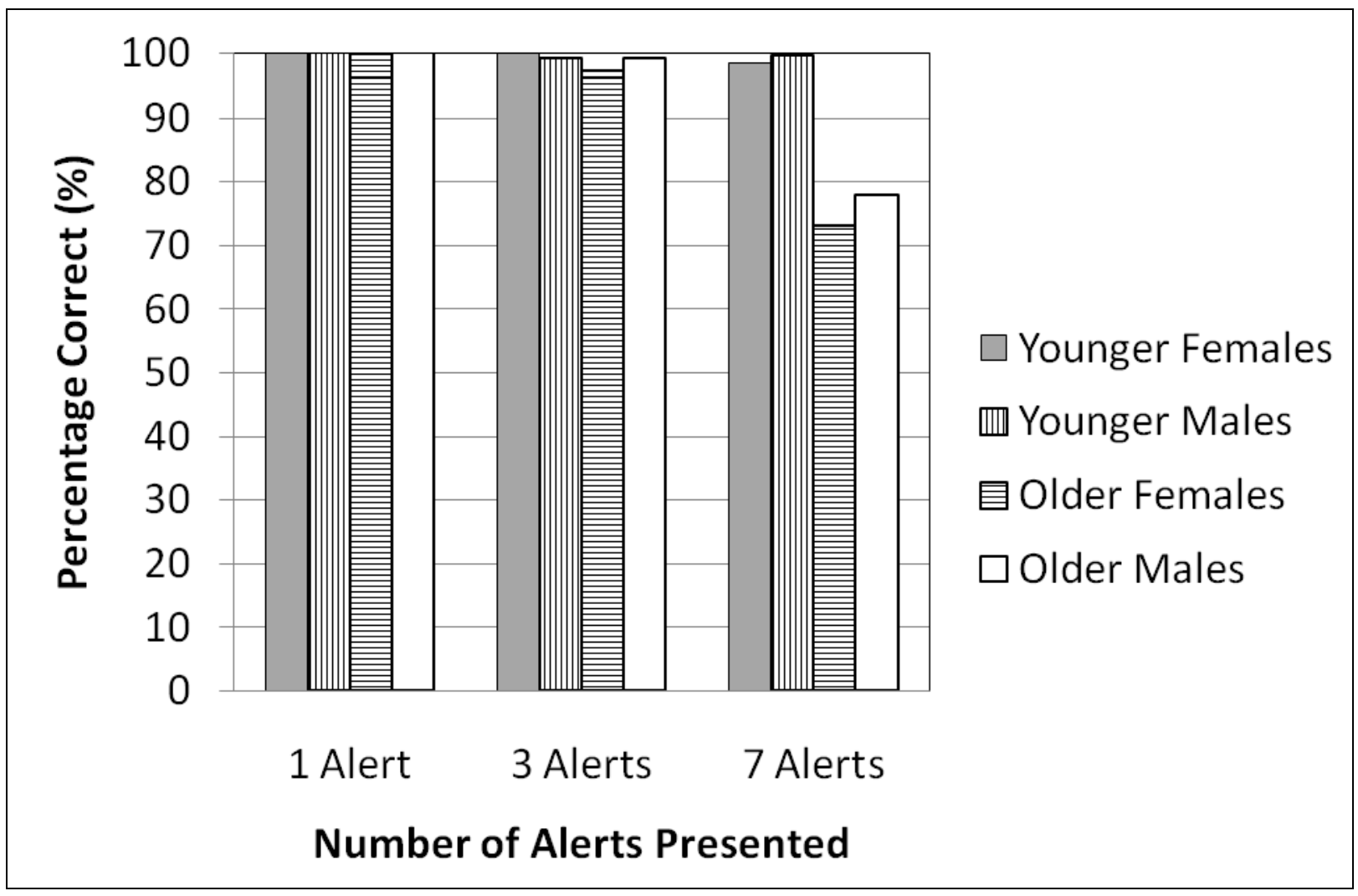

Figure 27. Drivers' verbal response accuracy.

A significant difference in performance between males and females was not found $\left(\chi^{2}(1)\right.$ $=2.0872, p=0.1485$. Fisher's exact $p=0.1754)$. Females accurately identified 91 percent of the alerts, while males accurately identified 93 percent of the alerts.

\section{Driver Ratings}

This section analyzes drivers' assessments of the alert identification task difficulty. Drivers' ratings of task difficulty were measured using six questions. Statistical analyses of their responses are presented below. The interested reader is referred to Appendix $\mathrm{H}$ for the results of drivers' opinions regarding the alerts' design.

\section{Question 1: Please rate how difficult it was to make the correct driving action for each vibration alert.}

A 2 (Age) x 2 (Gender) x 3 (\#Alerts) mixed factors ANOVA was performed using drivers' ratings of how difficult it was to perform the correct driving action as the dependent variable. Here, drivers used the rating scale to select a number that best reflected their response. Drivers were informed that half numbers (e.g., 4.5) were acceptable. The number of alerts was found to be a significant main effect $(\mathrm{F}(2,40)=$ $22.21, p<0.0001)$. On average, drivers found it very easy to make the correct driving action when only one alert was presented (mean response $=6.46$, s.e. $=0.10$ ). They found it easy when three alerts were presented (mean response $=5.48$, s.e. $=0.26$ ) as well 
as when seven alerts were presented (mean response $=4.71$, s.e. $=0.25$ ). A Tukey (a) multiple comparisons test revealed that drivers' mean difficulty rating of one alert was significantly different from their mean difficulty rating of three alerts (Adjusted $p=$ 0.0017 ), as well as seven alerts (Adjusted $p<0.0001$ ). Drivers' mean difficulty rating of three alerts was significantly different from their mean difficulty rating of seven alerts (Adjusted $p=0.0151)$. Drivers' age was also found to be a significant main effect ( $\mathrm{F}(1$, $20)=6.7, p=0.0176)$. On average, younger drivers found it very easy to make the correct driving action (mean response $=5.93$, s.e. $=0.21$ ), while older drivers found it easy to make the correct driving action (mean response 5.17 , s.e. $=0.21$ ). Figure 28 presents drivers' mean responses broken down by their age and gender. It can be seen that drivers generally found it harder to make the correct driving action as the number of alerts presented in a condition increased.

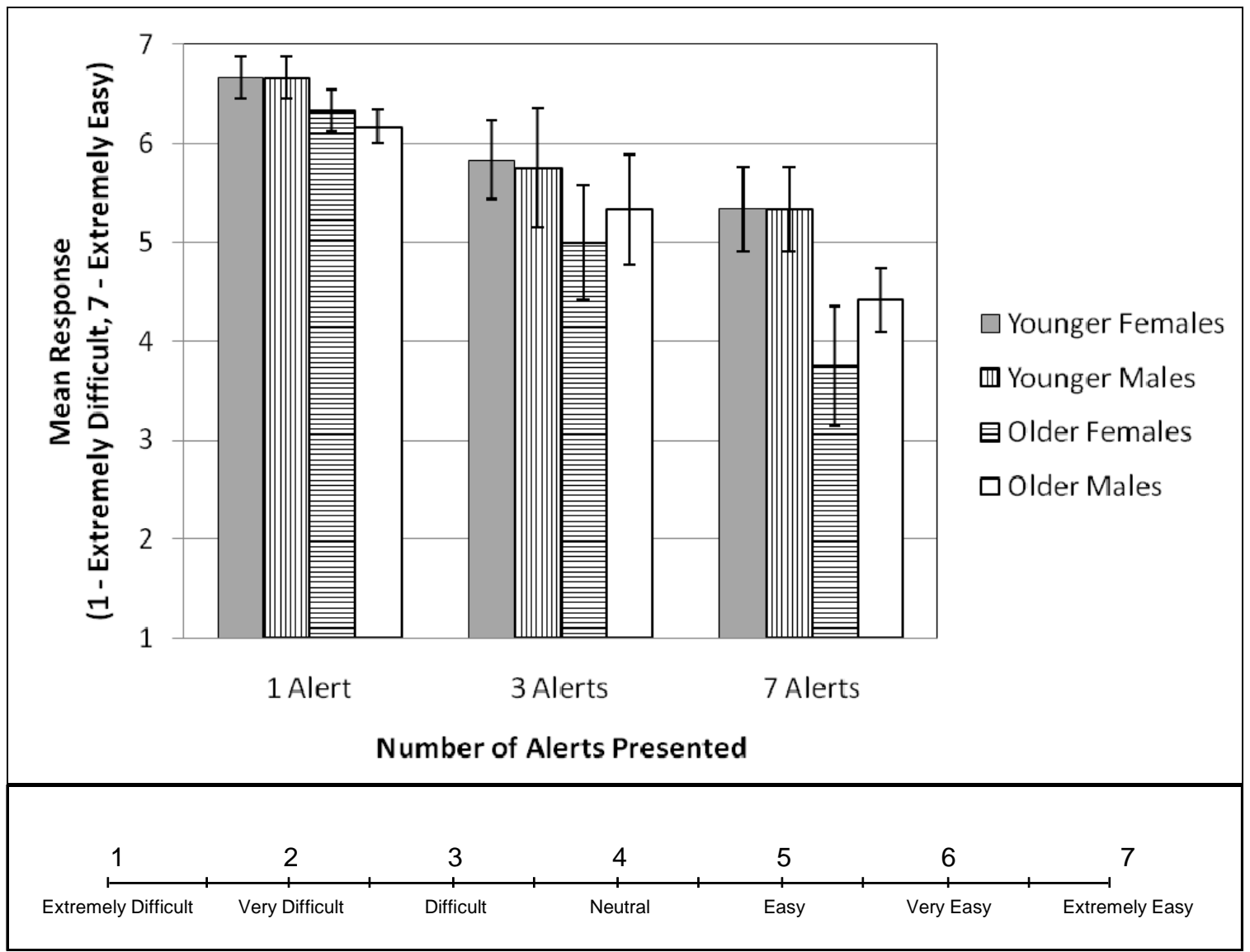

Figure 28. Drivers' ratings of how difficult it was to make the correct driving action for each vibration alert. 


\section{Question 2: Please rate how difficult it was to identify the vibration alerts.}

A 2 (Age) x 2 (Gender) x 3 (\#Alerts) mixed factors ANOVA was performed using drivers' ratings of how difficult it was to verbally identify the vibration alerts as the dependent variable. The number of alerts was found to be a significant main effect $(\mathrm{F}(2$, $40)=36.25, p<0.0001)$. On average, drivers found it very easy to identify the vibration alerts when only one alert was presented (mean response $=6.46$, s.e. $=0.15$ ). They found it very easy to identify the alerts when three alerts were presented (mean response $=6.00$, s.e. $=0.19$ ). Drivers had a neutral response when seven alerts were presented (mean response $=4.27$, s.e. $=0.27$ ). A Tukey (a) multiple comparisons test revealed that drivers' mean difficulty rating of seven alerts was significantly different from their mean difficulty rating of one alert (Adjusted $p<0.0001$ ), as well as three alerts (Adjusted $p<$ $0.0001)$. Drivers' age was also found to be a significant main effect $(\mathrm{F}(1,20)=5.57, p=$ $0.0285)$. On average, younger drivers found it very easy to identify the vibration alerts (mean response $=5.87$, s.e. $=0.18$ ), while older drivers found it easy to identify the alerts (mean response 5.28, s.e. $=0.18$ ). Figure 29 presents drivers' mean response broken down by their age and gender. It can be seen that drivers generally found it harder to verbally identify the alerts as the number of alerts increased.

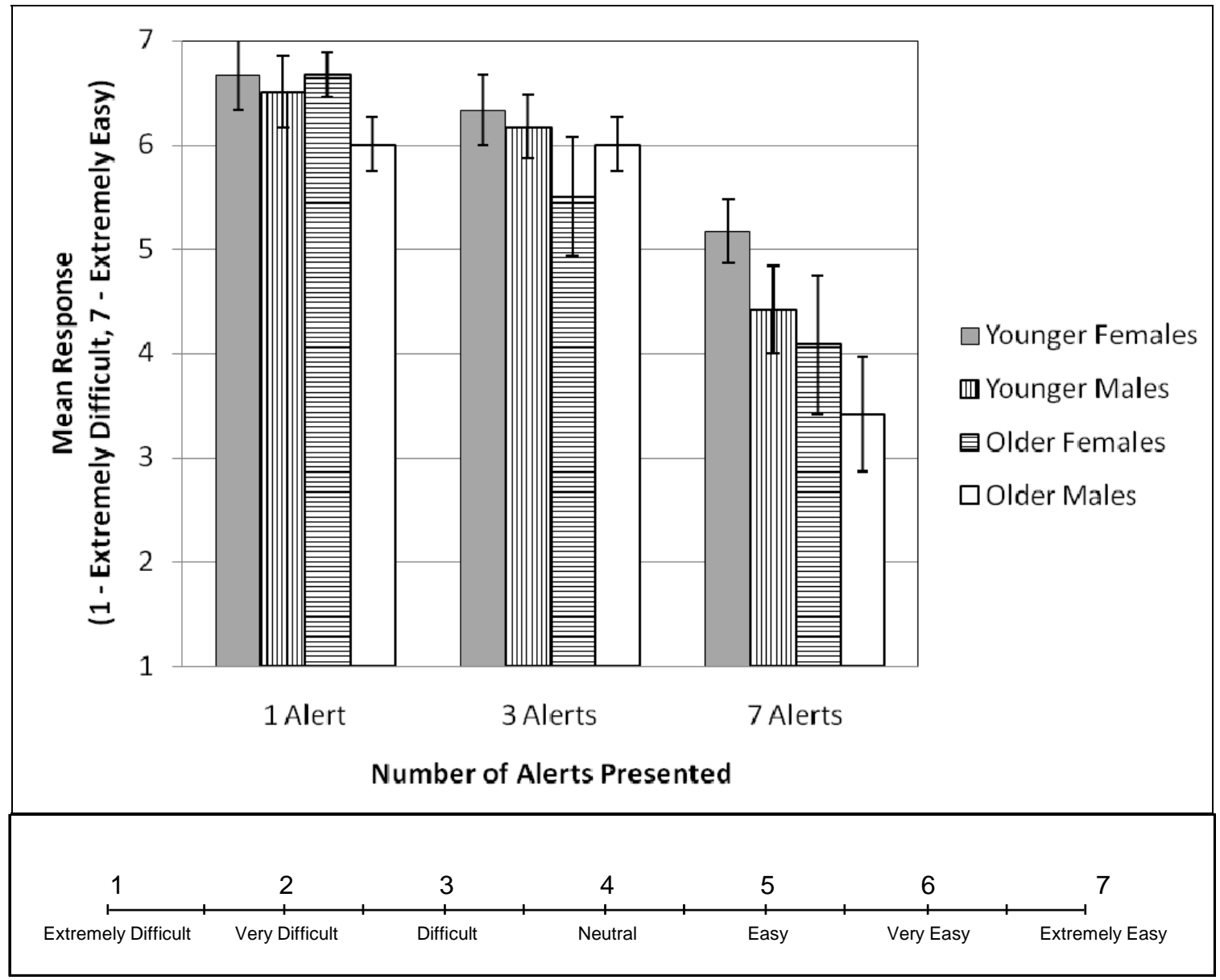

Figure 29. Drivers' mean ratings of how difficult it was to identify the vibration alerts. 


\section{Question 3: Please rate how confusing the vibration alerts were.}

A 2 (Age) x 2 (Gender) x 3 (\#Alerts) mixed factors ANOVA was performed using drivers' ratings of how confusing the vibration alerts were as the dependent variable. The number of alerts was found to be the only significant effect $(F(2,40)=13.19, p<$ 0.0001 ). On average, drivers rated the vibration alerts as very understandable when one alert was presented $($ mean response $=1.71$, s.e. $=0.17)$ as well as when three alerts were presented (mean response $=2.27$, s.e. $=0.27$ ). Drivers rated the alerts as understandable when seven alerts were presented (mean response $=3.5$, s.e. $=0.29$ ). A Tukey (a) multiple comparisons test revealed that drivers' mean confusion rating of seven alerts was significantly different from their mean confusion rating of one alert (Adjusted $p<$ 0.0001 ), as well as three alerts (Adjusted $p=0.0038$ ). Figure 30 presents drivers' mean responses broken down by their age and gender. It can be seen that drivers generally found the alerts to be less understandable as the number of alerts presented increased.

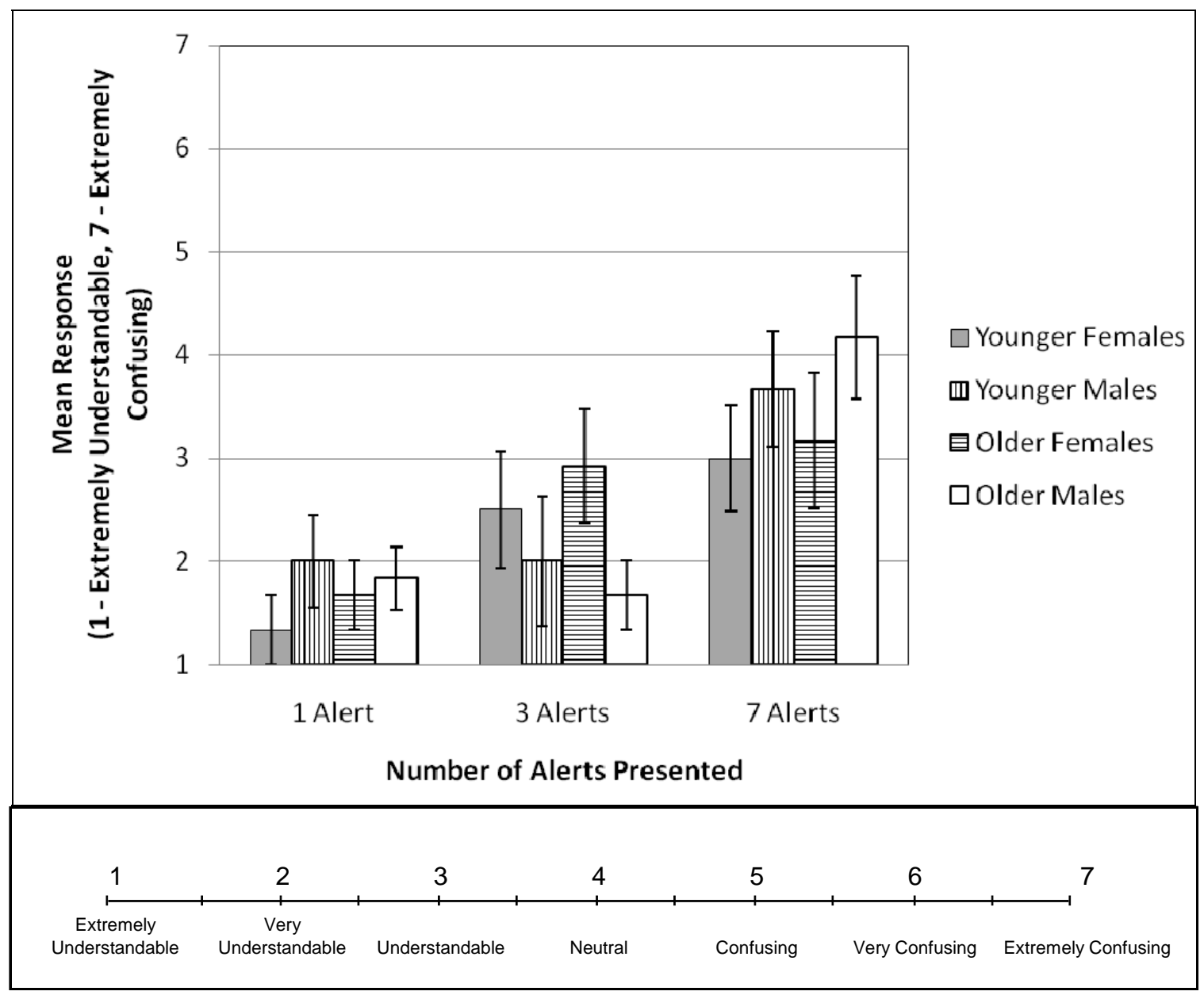

Figure 30. Drivers' mean ratings of how confusing the alerts were. 


\section{Question 4: Please rate how difficult it was to perform the task in Experiment 1.}

A 2 (Age) x 2 (Gender) x 3 (\#Alerts) mixed factors ANOVA was performed using drivers' ratings of how difficult it was to perform the overall task in Experiment 1 as the dependent variable. The number of alerts was found to be a significant main effect $(\mathrm{F}(2$, $40)=21.56, p<0.0001)$. On average, drivers found the task of manually and verbally identifying the alerts to be very easy when one alert was presented (mean response $=$ 6.50 , s.e. $=0.13$ ) as well as when three alerts were presented (mean response $=5.73$, s.e. $=0.26$ ). Drivers found the task to be easy when seven alerts were presented (mean response $=4.77$, s.e. $=0.28$ ). A Tukey (a) multiple comparisons test revealed that drivers' mean difficulty rating of one alert was significantly different from their mean difficulty rating of three alerts (Adjusted $p=0.0154$ ), as well as seven alerts (Adjusted $p$ $<0.0001)$. Drivers' mean difficulty rating of three alerts was also significantly different from their mean difficulty rating of seven alerts (Adjusted $p=0.0022$ ). Drivers' age was found to be a significant main effect $(\mathrm{F}(1,20)=6.63, p=0.0181)$. On average, younger drivers found it very easy to identify the vibration alerts (mean response $=6.10$, s.e. $=$ 0.24 ), while older drivers found it easy to identify the alerts (mean response 5.24, s.e. $=$ 0.24). Figure 31 presents drivers' mean responses broken down by their age and gender. It can be seen that drivers generally found the task in Experiment 1 to be harder as the number of alerts presented increased.

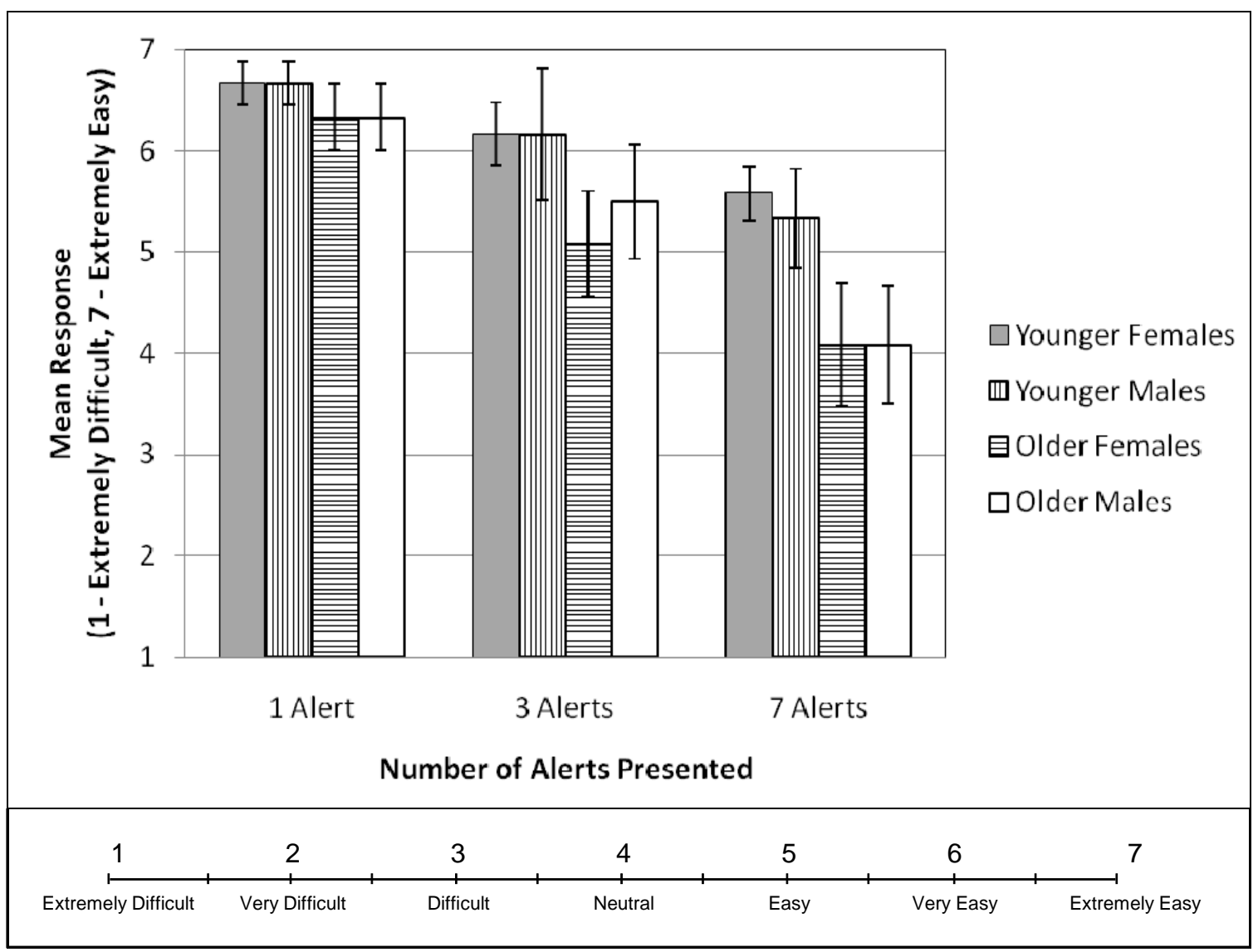

Figure 31. Drivers' mean ratings of how difficult it was to perform the manual and verbal response task in Experiment 1. 


\section{Question 5: Please rate how distinct the vibration alerts were from each other.}

A 2 (Age) x 2 (Gender) x 3 (\#Alerts) mixed factors ANOVA was performed using drivers' ratings of how distinct the vibration alerts were as the dependent variable. The number of alerts was found to be a significant main effect $(\mathrm{F}(2,40)=9.27, p=0.0005)$. On average, drivers rated the vibration alerts as very well-defined when one alert was presented (mean response $=6.08$, s.e. $=0.23$ ) as well as when three alerts were presented (mean response $=5.71$, s.e. $=0.30)$. Drivers rated the alerts as well-defined when seven alerts were presented (mean response $=4.52$, s.e. $=0.33$ ). A Tukey (a) multiple comparisons test revealed that drivers' mean distinction rating of seven alerts was significantly different from their mean distinction rating of one alert (Adjusted $p=$ 0.0005 ), as well as three alerts (Adjusted $p=0.0089$ ). Drivers' age was also found to be a significant main effect $(\mathrm{F}(1,20)=5.57, p=0.0219)$. On average, younger drivers found the alerts to be very well-defined (mean response $=5.89$, s.e. $=0.26$ ), while older drivers found them to be well-defined (mean response 4.99 , s.e. $=0.26$ ). Figure 32 presents drivers' mean responses broken down by their age and gender. It can be seen that younger drivers found the alerts to be more well-defined than older drivers did when three or seven alerts were presented.

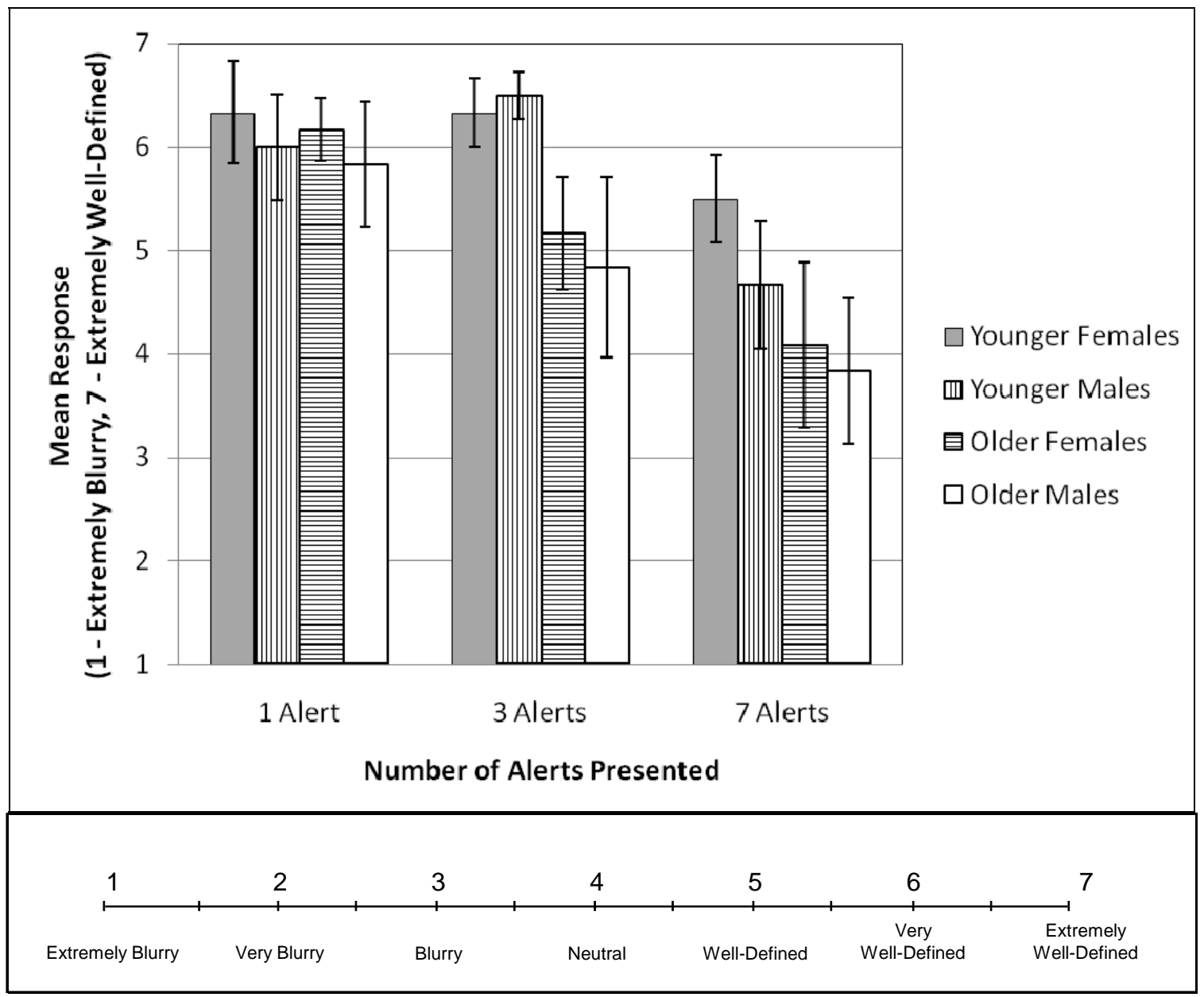

Figure 32. Drivers' mean rating of how distinct the alerts were. 
Question 6: Please rate how appropriate it was for the seat to vibrate like this when intended to alert you to a crash threat.

A 2 (Age) x 2 (Gender) x 3 (\#Alerts) mixed factors ANOVA was performed using drivers' ratings of how appropriate it was for the driver seat to vibrate as the dependent variable. The number of alerts was found to be a significant main effect $(F(2,40)=$ $10.81, p=0.0002)$. On average, drivers rated the haptic driver seat as very appropriate when only one alert was presented (mean response $=6.33$, s.e. $=0.15$ ), while drivers rated the haptic driver seat as appropriate when three alerts were presented (mean response $=5.23$, s.e. $=0.32$ ) and when seven alerts were presented (mean response $=$ 5.00 , s.e. $=0.30$ ). A Tukey (a) multiple comparisons test revealed that drivers' mean rating of one alert was significantly different from their mean rating of three alerts (Adjusted $p=0.0024$ ), as well as seven alerts (Adjusted $p<0.0003$ ). A significant Age $\mathrm{x}$ \#Alerts interaction was also found $(\mathrm{F}(2,40)=3.48, p=0.0403)$. Older drivers' mean ratings of the appropriateness of one alert was significantly different from their mean ratings of the appropriateness of seven alerts (Adjusted $p=0.0005$ ). Older drivers' mean ratings of the appropriateness of seven alerts was significantly different from younger drivers' mean ratings of the appropriateness of one alert (Adjusted $p=0.0005$ ). Figure 33 presents drivers' mean responses broken down by their age and gender.

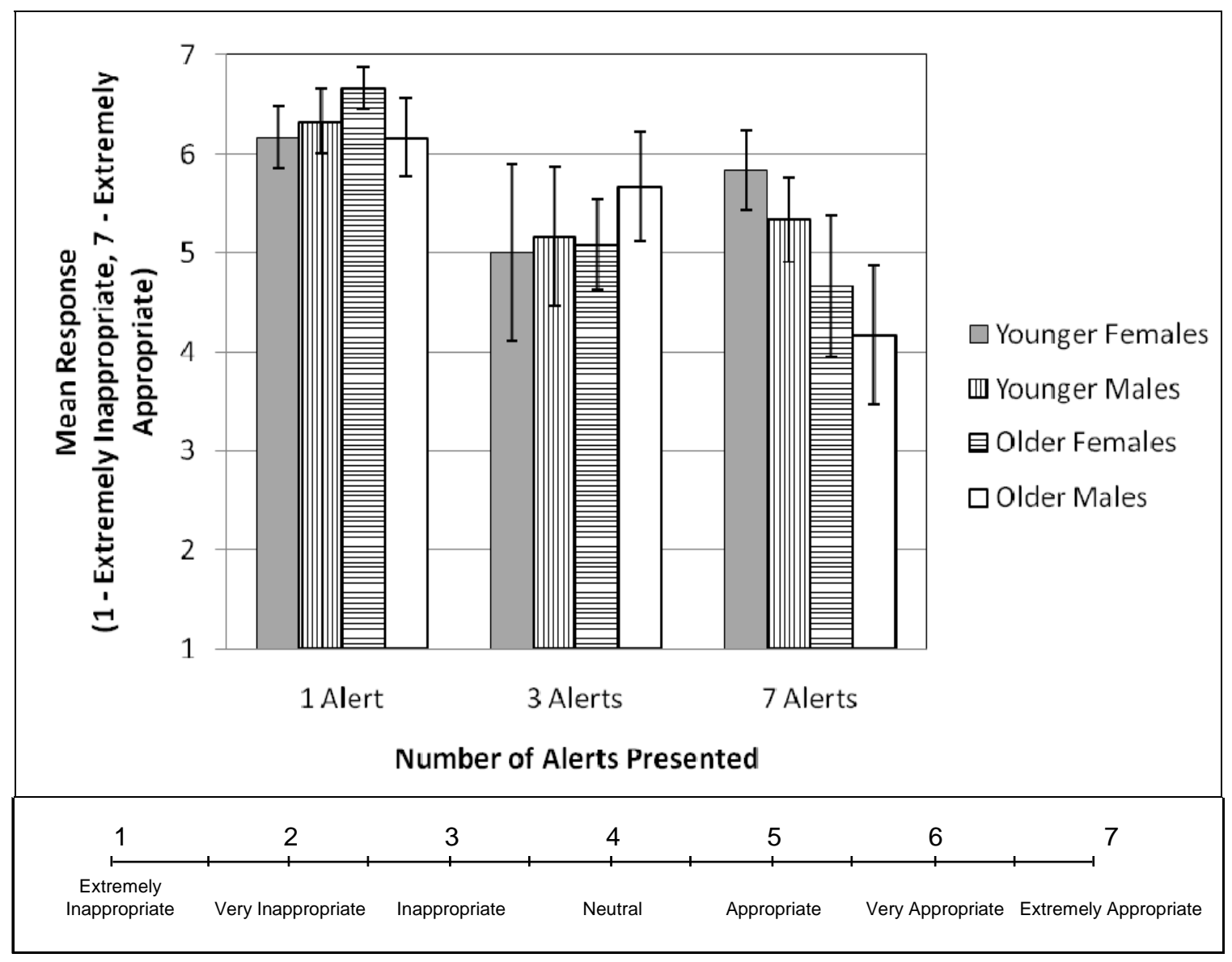

Figure 33. Drivers' mean ratings of how appropriate it was for the driver seat to vibrate when alerting drivers to a crash threat. 


\section{DISCUSSION}

The number of alerts that can be communicated to drivers through a haptic seat is an important design issue that requires consideration of human factors. The Hick-Hyman law of choice response time (Hick, 1952; Hyman, 1953) predicts that as the number of alternatives available for selection increases, humans' response time to any one alternative will increase. Accordingly, implementing multiple CASs in a vehicle's active safety system architecture may show a detrimental effect on the safety benefits if the time drivers take to perceive, cognitively process, and appropriately respond to an alert is delayed because of limitations in their information processing capabilities.

The purpose of this experiment was to quantify the effects of increasing the number of alerts presented to drivers through a haptic driver seat. Drivers' performance was analyzed in terms of manual response time and accuracy, as well as drivers' verbal response accuracy. In general, drivers performed the correct driving maneuver in response to the alerts. From a practical perspective, drivers correctly responded to one, three, and seven alerts with more than 94 percent accuracy. Drivers correctly identified 100 percent of the alerts when one alert could be presented, they identified 99 percent of alerts when three alerts could be presented, and they identified 97 percent of the alerts when seven alerts could be presented. These differences were found to be statistically significant. However, they are not considered to be fundamentally different. One reason why drivers may have been able to perform the correct manual responses to the alerts may be because of the intuitive design layout of the haptic driver seat. A strong stimulusresponse compatibility was established by mapping vibrations along the front edge of the driver seat to pressing the brake pedal, and mapping vibrations along the side edges of the seat to steering in the opposite direction. Even though the presentation of multiple alerts could be expected to confuse drivers, the consistent use of just three stimulus-response mappings appeared to have allowed drivers to effectively manage the manual response task by focusing on the location of the vibrations, rather than the specific vibration patterns. It is worth mentioning that drivers only experienced each alert type two times in a practice session prior to commencing the experiment. Hence, the performance results suggest that drivers did not take long to learn the haptic seat alerts.

Drivers' manual response time to the alerts was found to significantly increase as the number of alerts increased. From a practical perspective, drivers took an average of $0.831 \mathrm{~s}$ (s.e. $=0.031 \mathrm{~s}$ ) to respond to one alert, $0.847 \mathrm{~s} \mathrm{(s.e.}=0.017 \mathrm{~s}$ ) to respond to three alerts (a two percent increase), and $1.087 \mathrm{~s}(\mathrm{~s} . \mathrm{e} .=0.019 \mathrm{~s}$ ) to respond to seven alerts (a 31 percent increase) when the correct manual response was made. Drivers' mean manual response time to seven alerts significantly differed from their mean manual response time to one and three alerts. Further inspection found that drivers' mean BRTs to just the FCW alert increased as the number of alerts increased. Table 9 summarizes the observed changes in drivers' BRTs to the FCW alert. It also presents the additional distance covered by a vehicle travelling at $70 \mathrm{~km} / \mathrm{h}(44 \mathrm{mph})$ prior to drivers pressing the brake pedal. It can be seen that drivers' mean BRTs to three alerts did not meaningfully differ from their mean BRTs to one alert. Here, older females exhibited the largest change in mean BRT $(0.162 \mathrm{~s})$, while the other participant groupings exhibited mean BRT changes that were half this value. However, drivers' mean BRTs to seven alerts were 
substantially longer than their mean BRTs to one alert. It is particularly worth noting that older females' mean BRT increased markedly more than the other drivers. On the other hand, younger male drivers' mean BRT did not meaningfully lengthen, suggesting that the seven-alert approach may benefit a segment of the driving population. The utility of the seven-alert approach is discussed further in the future research section.

Table 9. Changes in Drivers' Mean Practical Manual Response Times

\begin{tabular}{|c|c|c|c|c|c|c|c|c|c|}
\cline { 2 - 10 } \multicolumn{1}{c|}{} & \multicolumn{2}{c|}{$\mathbf{1}$ Alert $\rightarrow \mathbf{3}$ Alerts } & \multicolumn{2}{c|}{ 3 Alerts $\rightarrow 7$ Alerts } & \multicolumn{2}{c|}{ 1 Alert $\rightarrow 7$ Alerts } \\
\cline { 2 - 10 } & $\begin{array}{c}\text { Difference } \\
(\mathrm{s})\end{array}$ & $\Delta \%$ & $\begin{array}{c}\Delta \text { Distance } \\
(\mathrm{m})\end{array}$ & $\begin{array}{c}\text { Difference } \\
(\mathrm{s})\end{array}$ & $\Delta \%$ & $\begin{array}{c}\Delta \text { Distance } \\
(\mathrm{m})\end{array}$ & $\begin{array}{c}\text { Difference } \\
(\mathrm{s})\end{array}$ & $\Delta \%$ & $\begin{array}{c}\Delta \text { Distance } \\
(\mathrm{m})\end{array}$ \\
\hline $\begin{array}{c}\text { Older } \\
\text { Females }\end{array}$ & 0.162 & $18 \%$ & 3.14 & 0.476 & $44 \%$ & 9.25 & 0.637 & $70 \%$ & 12.39 \\
\hline $\begin{array}{c}\text { Older } \\
\text { Males }\end{array}$ & -0.091 & $-11 \%$ & -1.77 & 0.326 & $43 \%$ & 6.34 & 0.235 & $28 \%$ & 4.57 \\
\hline $\begin{array}{c}\text { Younger } \\
\text { Females }\end{array}$ & 0.063 & $9 \%$ & 1.22 & 0.089 & $11 \%$ & 1.74 & 0.152 & $21 \%$ & 2.96 \\
\hline $\begin{array}{c}\text { Younger } \\
\text { Males }\end{array}$ & -0.073 & $-9 \%$ & -1.42 & 0.086 & $11 \%$ & 1.67 & 0.013 & $2 \%$ & 0.25 \\
\hline
\end{tabular}

A speed-accuracy tradeoff analysis was performed on drivers' theoretical response performance to the alerts. It was found that younger drivers were very efficient at processing one, three, and seven alerts. Older drivers, on the other hand, particularly older females, became less efficient as the number of alerts increased to seven. This suggests that the working memory decrements that come with age affected older drivers' performance as the number of alerts increased. It is noteworthy that younger drivers maintained their performance levels as the number of alerts increased.

Drivers' verbal response accuracy significantly degraded as the number of alerts increased. Drivers comprehended 100 percent of the alerts when one alert could be presented, 99 percent of the alerts when three alerts could be presented, and 87 percent of the alerts when seven alerts could be presented. However, performance decrements differed by age. Younger drivers comprehended one, three, and seven alerts, while older drivers were only able to comprehend one and three alerts. Older drivers exhibited a significant decrement in alert comprehension as the number of alerts increased to seven. This is because drivers had to memorize which patterns were associated with each alert. Limitations in older drivers' working memory likely prevented them from excelling at this task. An interesting finding from this study is that although older drivers had difficulty comprehending seven alerts, they were able to make the proper manual response with over 94 percent accuracy. This suggests that had these drivers received an alert when an actual crash threat was present, they may still be able to make the correct avoidance maneuver. Perhaps these drivers developed an automatic response to the alerts.

Although drivers made the correct driving response to the alerts, the questionnaire data 
indicate that drivers found the manual and verbal response tasks to become increasingly difficult as the number of alerts expanded from one to seven. Boehm-Davis, et al. (2007) advise practitioners to consider users' ratings of task difficulty even when objective task performance does not differ, as workload may play a significant role in circumstances of critical safety. Drivers' perceptions of the manual task difficulty, however, did align with their manual response time scores, where larger differences were observed as the number of alerts increased from three to seven. Furthermore, drivers' perceptions of the verbal task difficulty were on par with their verbal response performance, again where larger differences were observed as the number of alerts increased from three to seven. It is worth pointing out that drivers' harshest ratings of either the manual or verbal response tasks were moderate (this may be an artifact of participants not wanting to harshly criticize the study). Only a few older drivers rated the manual response task as difficult when seven alerts were presented. The other drivers were either neutral or found the tasks to be easy. A similar trend was observed for drivers' ratings of the verbal response task difficulty. Furthermore, when asked how confusing the alerts were, the majority of drivers either rated the seven-alert approach as understandable, or were neutral in their opinions. In general, drivers' ratings did not indicate that the manual or verbal response tasks were unmanageable.

By considering drivers' manual response accuracy and time, their verbal response performance, as well as their ratings of the experimental tasks, an understanding of the number of alerts an integrated CAS should adopt can begin to be established. The findings from this dissertation suggest that an integrated CAS should adopt a three-alert approach. This is because this was the most functional design in which drivers remained able to quickly initiate the correct driving maneuver, fully comprehend the meaning of the alerts, and not find the identification task to be difficult. Adopting a three-alert approach avoids the performance decrements that drivers exhibited when experiencing the seven-alert approach. Although drivers were able to make the correct driving response in the seven-alert approach, they took significantly longer, failed to comprehend all of the alerts, and rated the task to be harder. These degradations may implicate drivers' crash avoidance performance, particularly when stressed by their safety being jeopardized. At the same time, younger drivers performance with the seven-alert approach did not degrade as much as older drivers' performance did, and so further research with the seven-alert approach is warranted.

Although the three-alert and seven-alert alert approaches differed according to the amount of information they conveyed, they also differed in that each alert in the threealert approach was presented through a unique location, while the alerts in the seven-alert approach were presented through common tactors. Presenting alerts through unique tactors may facilitate improved response performance. Perhaps presenting some of the alerts in the seven-alert approach through a different location (e.g., presenting the left and right LDW alerts through a vibrating steering wheel) may facilitate faster response times. Additional research using the seven-alert approach should be performed before it is discounted as a viable option.

In determining whether CASs are appropriate, designers must address a tradeoff that 
exists between presenting alerts in which a percentage of the driving population will make the incorrect response to, versus not presenting alerts at all and allowing nature's course to unfold. An analysis that can be used to help address this tradeoff is the odds ratio. The odds ratio compares the odds of a crash being avoided with CAS feedback to the odds of a crash being avoided without CAS feedback. To compute the odds ratio, designers must first estimate:

- The likelihood of a crash being avoided without CAS feedback, $p$.

- The likelihood of a crash being avoided with CAS feedback, $q$.

Methods for estimating $p$ have been proposed by Battelle (2007), where the number of conflicts recorded in a heavy vehicle naturalistic driving study is related to the vehicle miles travelled to assess the likelihood of being involved in a conflict. Methods for estimating q were proposed by McLaughlin, Hankey, \& Dingus (2008). They involve inputting crash or near-crash data recorded in naturalistic driving studies into alert algorithms to determine when alerts would be generated. Drivers' response behavior to these alerts is then simulated by varying response parameters. The time available before a crash occurs is then used to estimate the percentage of the population that would be able to avoid the events. The odds ratio is then computed as

$$
\text { Odds ratio }=\frac{p(1-q)}{q(1-p)}
$$

An odds ratio greater than 1 indicates that drivers have a better chance at avoiding a collision with CAS feedback than they would without CAS feedback. Trading off erroneous responses for improved highway safety is worthwhile if the odds ratio is significantly large. It should be mentioned that estimates of $p$ and $q$ are difficult to obtain, even with existing naturalistic driving datasets. This is because of the limited conditions in which events are observed. Estimates of $p$ may improve with expansive naturalistic data collection efforts such as that planned by the second Strategic Highway Research Program (SHRP 2) (Transportation Research Board of the National Academies, 2008). The SHRP 2 project will be the largest naturalistic driving study ever conducted. Estimates of $q$ can also be improved using these expansive datasets. 


\section{CHAPTER 4. EXPERIMENT 2: EFFECTS OF HAPTIC SEAT ALERT LOCATION ON DRIVERS' ABILITY TO DIFFERENTIATE MEANINGFUL FROM UNINFORMATIVE ALERTS}

\section{PURPOSE}

The purpose of this experiment was to investigate whether evoking meaningful and uninformative haptic alerts through different locations in the driver seat improves drivers' response performance to the meaningful alerts compared to when both alert types are presented through the same seat location. Since drivers may ignore uninformative alerts, it is possible that meaningful alerts presented through the same seat location as the uninformative alerts will be ignored.

\section{METHOD}

The method used in Experiment 2 is presented in this section. Since the selected participants, test vehicle, test track, DAS, as well as the video recording hardware and software are the same as those used in Experiment 1, these aspects of the study are not described. However, since the haptic driver seat design and experimental procedure were changed, they are described below.

\section{Apparatus}

The haptic driver seat was programmed to vibrate in four areas to communicate four different alerts to drivers. The four tactors used were the two placed in the back-left and back-right corners of the seat pan, as well as two tactors mounted in the left- and rightside of the seat back. The four patterns that were used are listed in Table 10. The LDW and LCW alert patterns are the same as those used in Experiment 1. Again, the amount of time that the tactors vibrated for each pattern was controlled to be one second.

Table 10. Experiment 2 Vibrotactile Patterns

\begin{tabular}{|l|l|}
\hline \multicolumn{1}{|c|}{ Alert } & \multicolumn{1}{c|}{ Haptic Pattern } \\
\hline Left LCW Alert & Tactor pulses five times (200 ms on, 50 ms off) \\
\hline Right LCW Alert & Tactor pulses five times (200 ms on, 50 ms off) \\
\hline Left LDW Alert & Tactor activates for one second \\
\hline Right LDW Alert & Tactor activates for one second \\
\hline
\end{tabular}

\section{Procedure}

After completing Experiment 1, participants were informed that they would be performing a new experimental task. The in-vehicle experimenter instructed participants to drive the test vehicle at $20 \mathrm{mph}$ down the left or right lane. Participants were asked to make lane changes into the adjacent lane at specific points on the road. Participants were 
asked to use their turn signals, check their mirrors, and check their blind spots prior to and during the execution of the lane changes. Even though there was no other traffic on the road, participants cooperated and performed this routine. Participants were told that during each lane change, they may feel a haptic seat alert. They were told that when making lane changes they may experience a LDW alert, a LCW alert, or no alert at all. However, unlike Experiment 1, participants were told to only steer back into the original lane when they felt an LCW alert. Participants were told to continue making the lane change into the destination lane if they felt an LDW alert, or no alert at all. Participants were reminded that the LCW alert consisted of 5 pulses, while the LDW alert was a continuous vibration. Participants were told to not verbally identify the alerts.

Both the left LDW and LCW alerts were presented through the back-left corner of the driver seat during the "Seat Pan" condition. Likewise, the right LDW and LCW alerts were presented through the back-right corner of the driver seat. During the "Seat Pan + Seat Back" condition, however, the LDW alerts were presented through the seat corners, while the left and right LCW alerts were presented through the left and right tactors mounted in the seat back, respectively.

Participants experienced 28 alerts per condition. Half of these alerts were LCW alerts, while the other half were LDW alerts. An equal number of left- and right-side alerts were presented as well. As such, there was one practice trial and six experimental trials for each alert $((1+6) \times 4=28)$.

\section{Independent Variables}

This study consists of a 2 (Age) $\times 2$ (Gender) $\times 2$ (Side) $\times 2$ (Location) mixed factor design. The independent variables are described below.

\section{Age (Older, Younger) - Between-Subjects}

The same participants from Experiment 1 were used. The two age groups were 18-25 years old and above 65 years old.

\section{Gender (Female, Male) - Between-Subjects}

An equal number of females and males were selected to for this experiment.

\section{Side (Left, Right) - Within-Subjects}

Alerts were presented during left and right lane changes on the left and right sides, respectively. An equal number of left- and right-sided alerts were generated. Their presentation order was counterbalanced.

\section{Location (Same Location, Different Location) - Within-Subjects}

The location of the driver seat that vibrated was controlled at two levels. The order was counterbalanced. 


\section{Same Location}

The left and right LCW alerts were presented through the same tactors as the left and right LDW alerts. The LCW alerts were considered to be meaningful alerts because drivers were instructed to respond to them, while the LDW alerts were considered to be uninformative because drivers were instructed to ignore them. The LCW and LDW patterns used were the same as those used in Experiment 1.

\section{Different Location}

The left and right LCW alerts were presented through tactors mounted in the seat back rather than the seat pan. The left and right LDW alerts continued to be presented through the seat pan. Here, the meaningful and uninformative alerts were presented through different locations in the driver seat.

\section{Dependent Variables}

The effects of presenting meaningful and uninformative alerts through different locations in the driver seat were assessed using measures of driver response behavior to the alerts. These measures are described below.

\section{Manual Response Accuracy}

Participants were instructed to abort a lane change when they felt an LCW alert generated as they executed the lane change. Participants were also instructed to completely execute the lane change if they felt a LDW alert or no alert at all. Participants' ability to make the correct driving response based on the alert presented was scored. Similarly to Experiment 1, a practical manual response score was developed to not penalize participants for removing their foot from the throttle, pressing the brake pedal, or jerking the steering wheel to the left or right (where the vehicle's trajectory was not affected) in response to the haptic alerts. Table 11 identifies the driving maneuvers that were required, permitted, and not allowed for a trial to be scored as correct. 
Table 11. Permitted Maneuvers for the Practical Manual Accuracy Score

\begin{tabular}{|c|c|c|c|c|}
\hline $\begin{array}{c}\text { Manual } \\
\text { Response }\end{array}$ & LDW L & LDW R & LCW L & LCW R \\
\hline $\begin{array}{c}\text { Foot Off Throttle } \\
\text { Press Brake } \\
\text { Pedal }\end{array}$ & Allowed & Allowed & Allowed & Allowed \\
\hline $\begin{array}{c}\text { Steer } \\
\text { Left }\end{array}$ & Required & Not Allowed & Allowed & Allowed \\
\hline $\begin{array}{c}\text { Steer Right } \\
\text { Nerk }\end{array}$ & Not Allowed & Required & Required & Allowed \\
\hline $\begin{array}{c}\text { Left } \\
\text { Light }\end{array}$ & Allowed & Allowed & Allowed & Allowed \\
\hline
\end{tabular}

A theoretical manual response score was also developed to measure how drivers performed in this task if they were not permitted to jerk the steering wheel to the left or right when making a correct response. Similarly to Experiment 1, removing one's foot from the throttle and applying the brakes were permitted. Table 12 identifies the manual responses that were required, permitted, and not allowed for a trial to be scored as correct. 
Table 12. Permitted Maneuvers for the Theoretical Manual Accuracy Score

\begin{tabular}{|c|c|c|c|c|}
\hline $\begin{array}{c}\text { Manual } \\
\text { Response }\end{array}$ & LDW L & LDW R & LCW L & LCW R \\
\hline $\begin{array}{c}\text { Foot Off Throttle } \\
\text { Press Brake } \\
\text { Pedal }\end{array}$ & Allowed & Allowed & Allowed & Allowed \\
\hline $\begin{array}{c}\text { Steer } \\
\text { Left }\end{array}$ & Required & Not Allowed & Not Allowed & Required \\
\hline $\begin{array}{c}\text { Steer Right } \\
\text { Nerk }\end{array}$ & Not Allowed & Required & Required & Not Allowed \\
\hline $\begin{array}{c}\text { Left } \\
\text { Right }\end{array}$ & Not Allowed & Not Allowed & Not Allowed & Not Allowed \\
\hline
\end{tabular}

\section{Manual Response Time}

The elapsed time from an LCW alert to participants' initial steering wheel input was measured using the collected video footage described in Experiment 1. The initiation of the steering input could be easily assessed by stepping through the data frame-by-frame until the steering wheel input became observable.

\section{Driver Ratings}

Participants' perception of the experimental task's difficulty was measured after each condition. Appendices I and J present the questions that were asked.

\section{Hypotheses}

This experiment was designed under the hypotheses that drivers would be faster, and more accurate, at responding to the meaningful LCW alerts when they were presented through a different location in the driver seat than the uninformative LDW alerts compared to when both alert types were presented through the same location. It was also hypothesized that drivers would find the experimental task easier when the two alert 
types were separated compared to when they were presented through the same location. Older drivers were hypothesized to have longer response times and err more than younger drivers. Performance was not expected to differ according to the side of the seat the alerts were presented, or the participants' gender.

\section{RESULTS}

\section{Manual Response Accuracy}

This section presents the manual response accuracy results in terms of both practical accuracy and theoretical accuracy.

\section{Practical Manual Response Accuracy}

In general, drivers made fewer mistakes in responding to the alerts when they were presented through different locations compared to when they were presented through the same location. Drivers made the correct manual response 97 percent of the time when the alerts were presented through the same location, while they made the correct manual response 100 percent of the time when the alerts were presented through different locations. This difference was found to be statistically significant $(\mathrm{Q}(1)=15.0010, p<$ $0.0001)$.

Younger drivers always made the correct manual response, while older drivers made the correct manual response 96 percent of the time. This difference was found to be statistically significant $\left(\chi^{2}(1)=22.4283, p<0.0001\right.$. Fisher's exact $\left.p<0.0001\right)$. Statistically significant gender differences were also found $\left(\chi^{2}(1)=18.5358, p<0.0001\right.$. Fisher's exact $p<0.0001)$. Male drivers made the correct manual response 100 percent of the time, while female drivers made the correct manual response 96 percent of the time.

Closer inspection revealed that the Age and Gender effects mainly occurred because of older females making incorrect driving responses to the alerts. Figure 34 below shows that younger females, younger males, and older males did not have trouble performing the correct manual response despite the alerts' location. 


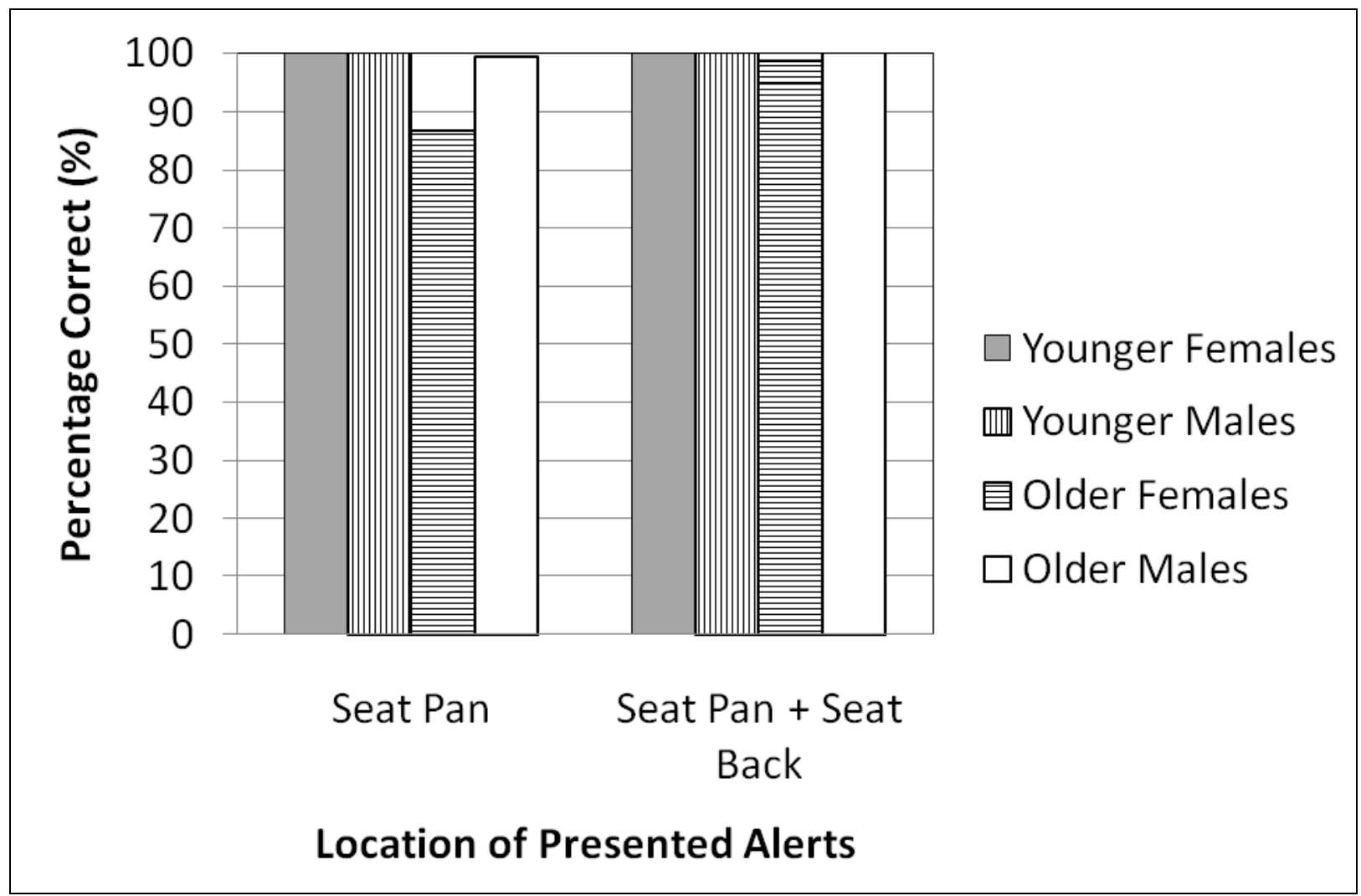

Figure 34. Drivers' practical manual response accuracy to alerts presented through just the seat pan, or both the seat pan and seat back.

Manual response accuracy was not found to statistically differ according to the side of the seat the alerts were presented $(\mathrm{Q}(1)=0.7408, p=0.3894)$. Drivers correctly identified the alerts 98 percent of the time when they were presented on either the left or right side.

In just considering drivers' response performance to the meaningful (LCW) alerts, it was found that drivers made fewer mistakes perceiving the meaningful alerts when they were presented through a different location from the uninformative (LDW) alerts compared to when they were both presented through the same location. Drivers made the correct manual response 99 percent of the time when the meaningful alerts were presented through the same location as the uninformative alerts, while they made the correct manual response 100 percent of the time when they were presented through a different location from the uninformative alerts. This difference was not found to be statistically significant $(\mathrm{Q}(1)=4.021, p=0.0448)$.

Younger drivers always made the correct manual response, while older drivers made the correct manual response 99 percent of the time. This difference was not found to be statistically significant $\left(\chi^{2}(1)=4.028, p=0.0448\right.$. Fisher's exact $\left.p=0.1237\right)$. A significant gender difference was also not found $\left(\chi^{2}(1)=1.007, p=0.3156\right.$. Fisher's exact $p=0.6237)$. Figure 35 below shows drivers' manual response accuracy to the meaningful alerts broken down by their age and gender. It was also found that the side 
that the alert was presented on did not have a statistically significant effect on manual response accuracy $(\mathrm{Q}(1)=1.0052, p=0.316)$.

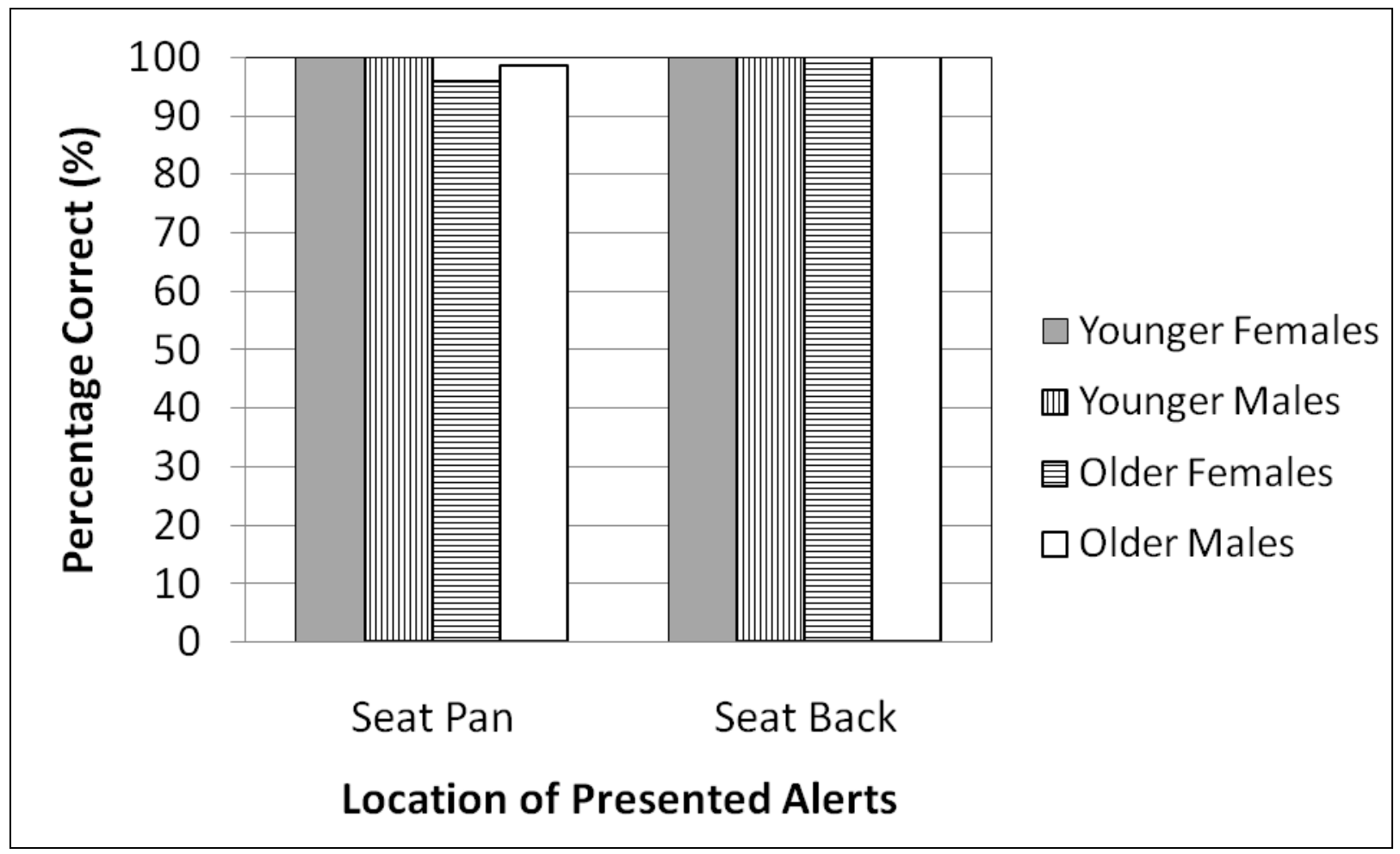

Figure 35. Drivers' practical manual response accuracy to just the meaningful alerts.

\section{Theoretical Manual Response Accuracy}

With respect to theoretical manual response accuracy, it was found that drivers made fewer mistakes in responding to the alerts when they were presented through a different location compared to when they were presented through the same location. Drivers made the correct manual response 86 percent of the time when the alerts were presented through the same location, while they made the correct manual response 93 percent of the time when the alerts were presented through different locations. This difference was found to be significant $(\mathrm{Q}(1)=13.832, p=0.0002)$.

Younger drivers made the correct manual response 92 percent of the time, while older drivers made the correct manual response 86 percent of the time. This difference was found to be significant $\left(\chi^{2}(1)=11.1498, p=0.0008\right.$. Fisher's exact $\left.p=0.0011\right)$. Significant gender differences were also found $\left(\chi^{2}(1)=16.8294, p<0.0001\right.$. Fisher's exact $p<0.0001)$. Male drivers made the correct manual response 93 percent of the time, while female drivers made the correct manual response 86 percent of the time.

Closer inspection revealed that the age and gender differences mainly occurred because older females as well as younger males made significantly more incorrect driving 
responses when the alerts were presented in the same location compared to when they were presented in different locations (Figure 36). Older females made the correct manual response 71 percent of the time when the alerts were presented in the same location, while they made the correct manual response 85 percent of the time when they were presented through different locations (this difference was found to be statistically significant, $\chi^{2}(1)=8.0357, p=0.0046$. Fisher's exact $\left.p=0.0068\right)$. Younger males made the correct manual response 88 percent of the time when the alerts were presented in the same location, while they made the correct manual response 95 percent of the time when they were presented through different locations (this difference was found to be statistically significant, $\chi^{2}(1)=5.3001, p=0.0213$. Fisher's exact $p=0.0346$ ). Statistically significant differences were not found between both older males' and younger females' performance in response to alerts presented in the same location compared to them being presented through different locations.

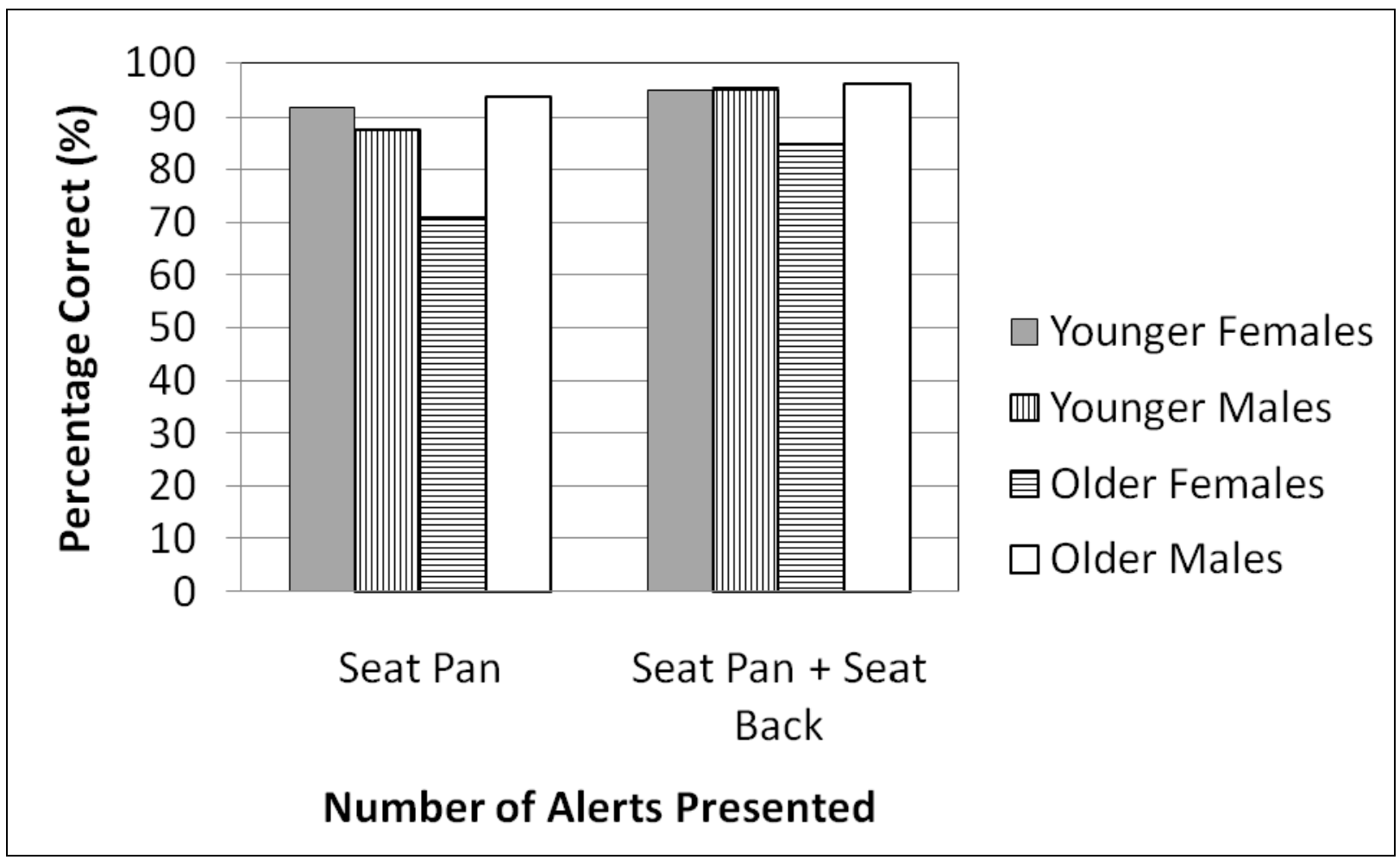

Figure 36. Drivers' theoretical manual response accuracy broken down by their age and gender.

Theoretical manual response accuracy was not found to differ by the side the alerts were presented on $(\mathrm{Q}(1)=0.0091, p=0.924)$. Drivers correctly identified the alerts 89 percent of the time when they were presented on either the left or right side.

In just considering drivers' response performance to the meaningful alerts, it was found that drivers made fewer mistakes perceiving the meaningful alerts when they were presented through a different location from the uninformative alerts compared to when they were both presented through the same location. Drivers made the correct manual 
response 98 percent of the time when the meaningful alerts were presented through the same location as the uninformative alerts, while they made the correct manual response 100 percent of the time when they were presented through a different location from the uninformative alerts. This difference was found to be statistically significant $(\mathrm{Q}(1)=$ 5.035, $p=0.0248)$. A significant Age effect was not found $\left(\chi^{2}(1)=1.8158, p=0.1778\right.$. Fisher's exact $p=0.3728$ ). A significant Gender effect was not found $\chi^{2}(1)=1.8158, p=$ 0.1778 . Fisher's exact $p=0.3728$ ). A statistically significant Side effect was also not found $\mathrm{Q}(1)=0.2014, p=0.6536$ ). Figure 37 shows drivers' manual response accuracy to the meaningful alerts broken down by their age and gender.

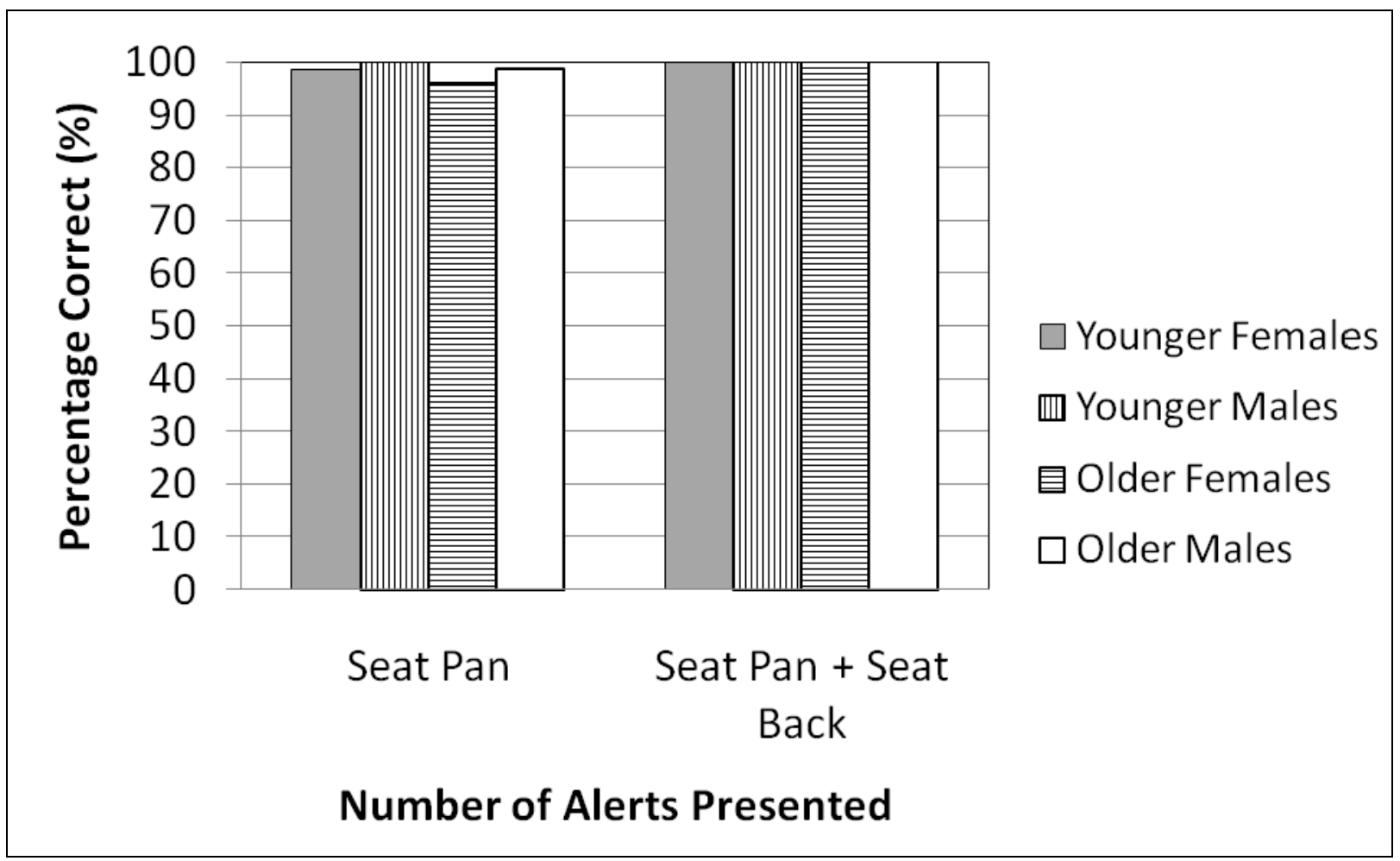

Figure 37. Drivers' theoretical manual response accuracy to just the meaningful alerts.

\section{Manual Response Time}

This section presents the manual response time results. Response time outliers were removed from the data prior to performing analyses. Appendix G documents which outliers were removed. The results in this section are presented in terms of both practical and theoretical manual response time.

\section{Practical Manual Response Time}

All response times observed when participants performed the correct practical manual response are analyzed in this section. Figure 38 shows the histogram of drivers' practical manual response times to the meaningful alerts when they were presented through the 
seat pan, as well as when they were presented through the seat back.

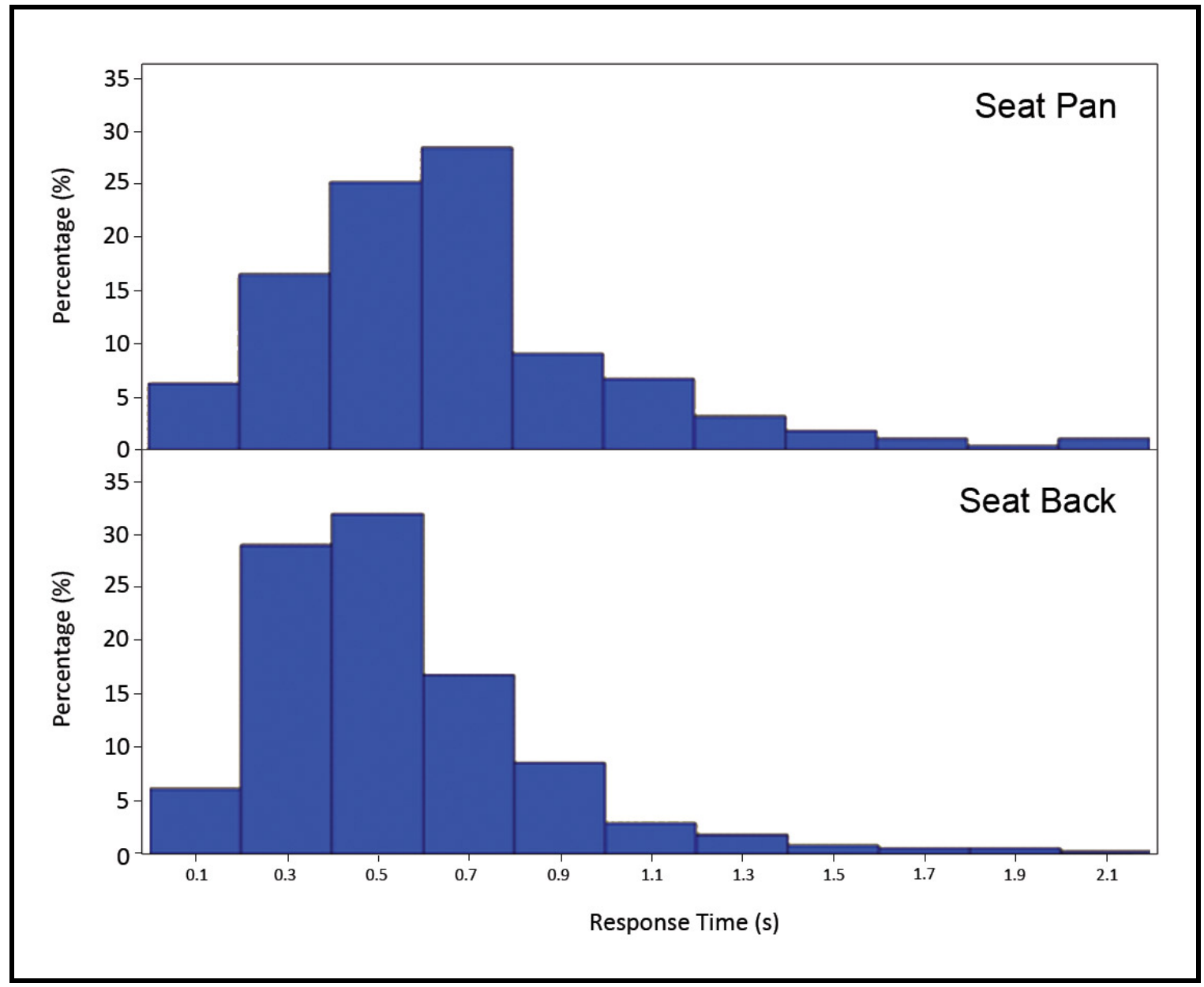

Figure 38. Practical manual response times to meaningful alerts presented through the seat pan, as well as through the seat back.

A 2 (Age) x 2 (Gender) x 2 (Side) x 2 (Location) mixed factors ANOVA was performed on the response time dependent variable. The location of the meaningful alerts was found to be a statistically significant main effect $(\mathrm{F}(1,20)=26.1, p<0.0001)$. Drivers' mean response time to the meaningful alerts presented through the seat pan was $0.666 \mathrm{~s}$ (s.e. $=0.021 \mathrm{~s}$ ), while their mean response time to meaningful alerts presented through the seat back was $0.522 \mathrm{~s}($ s.e. $=0.015 \mathrm{~s})$. A significant Age $\mathrm{x}$ Gender $\mathrm{x}$ Location interaction was also found $(\mathrm{F}(1,20)=4.96, p=0.0375)$. Older male drivers' mean response time to meaningful alerts presented through the seat pan was $0.752 \mathrm{~s}$ (s.e. $=$ $0.037 \mathrm{~s}$ ), while their mean response time to meaningful alerts presented through the seat back was $0.601 \mathrm{~s}$ (s.e. $=0.026 \mathrm{~s}$ ). A Tukey-Kramer multiple comparisons test found this difference to be statistically significant $(p=0.0302)$. Similarly, younger female drivers' mean response time to meaningful alerts presented through the seat pan was $0.727 \mathrm{~s}$ (s.e. $=0.034 \mathrm{~s}$ ), while their mean response time to meaningful alerts presented through the seat back was $0.562 \mathrm{~s}$ (s.e. $=0.023 \mathrm{~s}$ ). A Tukey-Kramer multiple comparisons test found this difference to be significant $(p=0.0225)$. These performance differences are shown in 
Figure 39. The side the alerts were presented on was found to be marginally significant $(\mathrm{F}(1,20)=4.42, p=0.0483)$. Drivers' mean manual response time to left $\mathrm{LCW}$ alerts was $0.594 \mathrm{~s}$ (s.e. $=0.018 \mathrm{~s}$ ), while their mean manual response time to right $\mathrm{LCW}$ alerts was $0.627 \mathrm{~s}(\mathrm{~s} . \mathrm{e} .=0.020 \mathrm{~s})$.

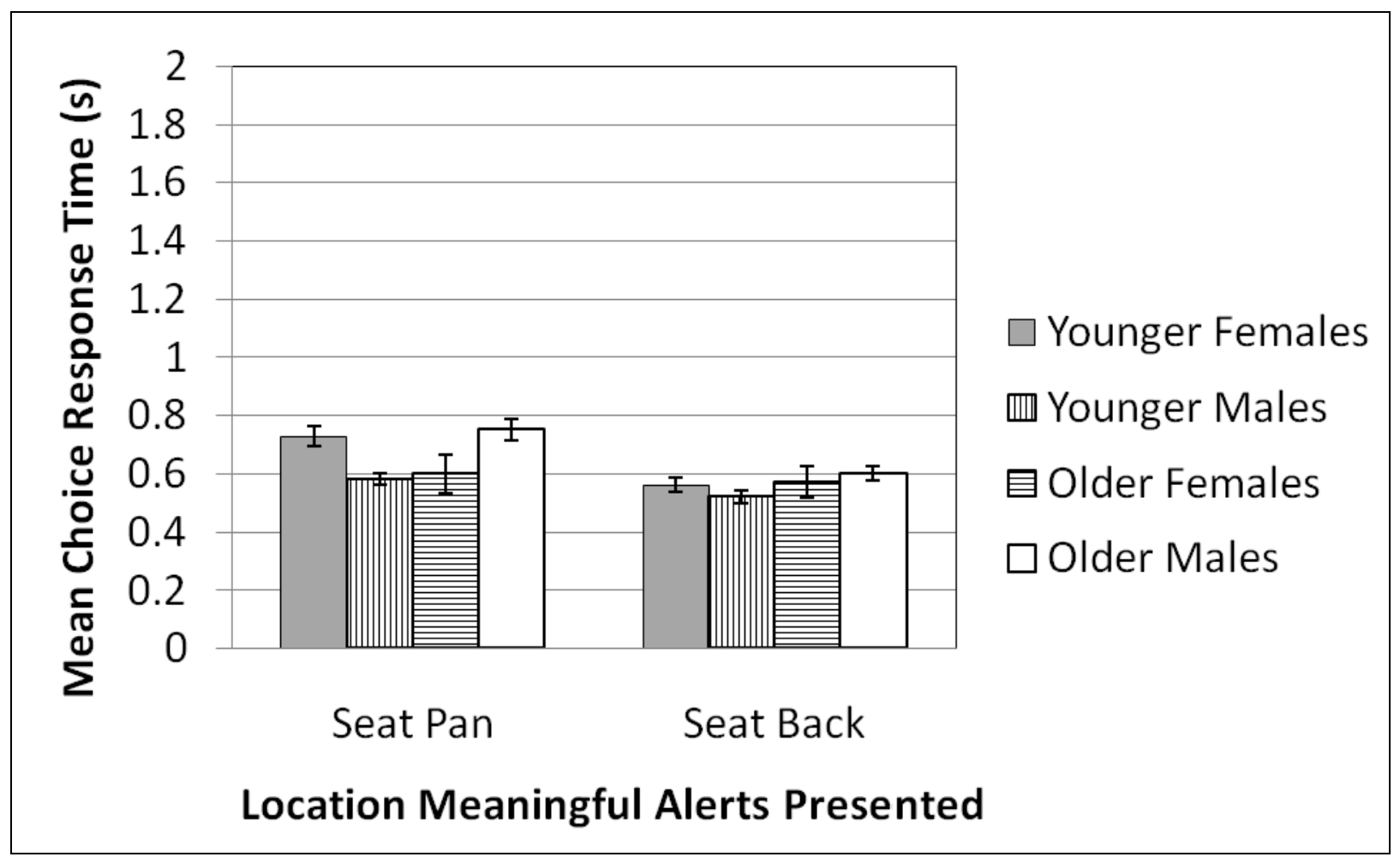

Figure 39. Drivers' mean practical manual response time to meaningful alerts.

The quantiles for driver's manual response times to meaningful alerts presented through the seat pan and seat back are shown in Table 13. Figure 40 presents drivers' $90^{\text {th }}$ percentile response times broken down by their age and gender. 
Table 13. Quantiles of Drivers' Manual Response Time to Meaningful Alerts

\begin{tabular}{|c|c|c|}
\hline Quantile & Seat Pan (s) & Seat Back (s) \\
\hline $100 \%$ Max & 2.100 & 2.000 \\
\hline $99 \%$ & 2.067 & 1.567 \\
\hline $95 \%$ & 1.333 & 1.000 \\
\hline $90 \%$ & 1.100 & 0.833 \\
\hline $75 \%$ Q3 & 0.800 & 0.633 \\
\hline $50 \%$ Median & 0.633 & 0.467 \\
\hline $25 \%$ Q1 & 0.433 & 0.367 \\
\hline $10 \%$ & 0.300 & 0.267 \\
\hline $5 \%$ & 0.200 & 0.200 \\
\hline $1 \%$ & 0.033 & 0.067 \\
\hline $0 \%$ Min & 0.033 & 0.033 \\
\hline
\end{tabular}

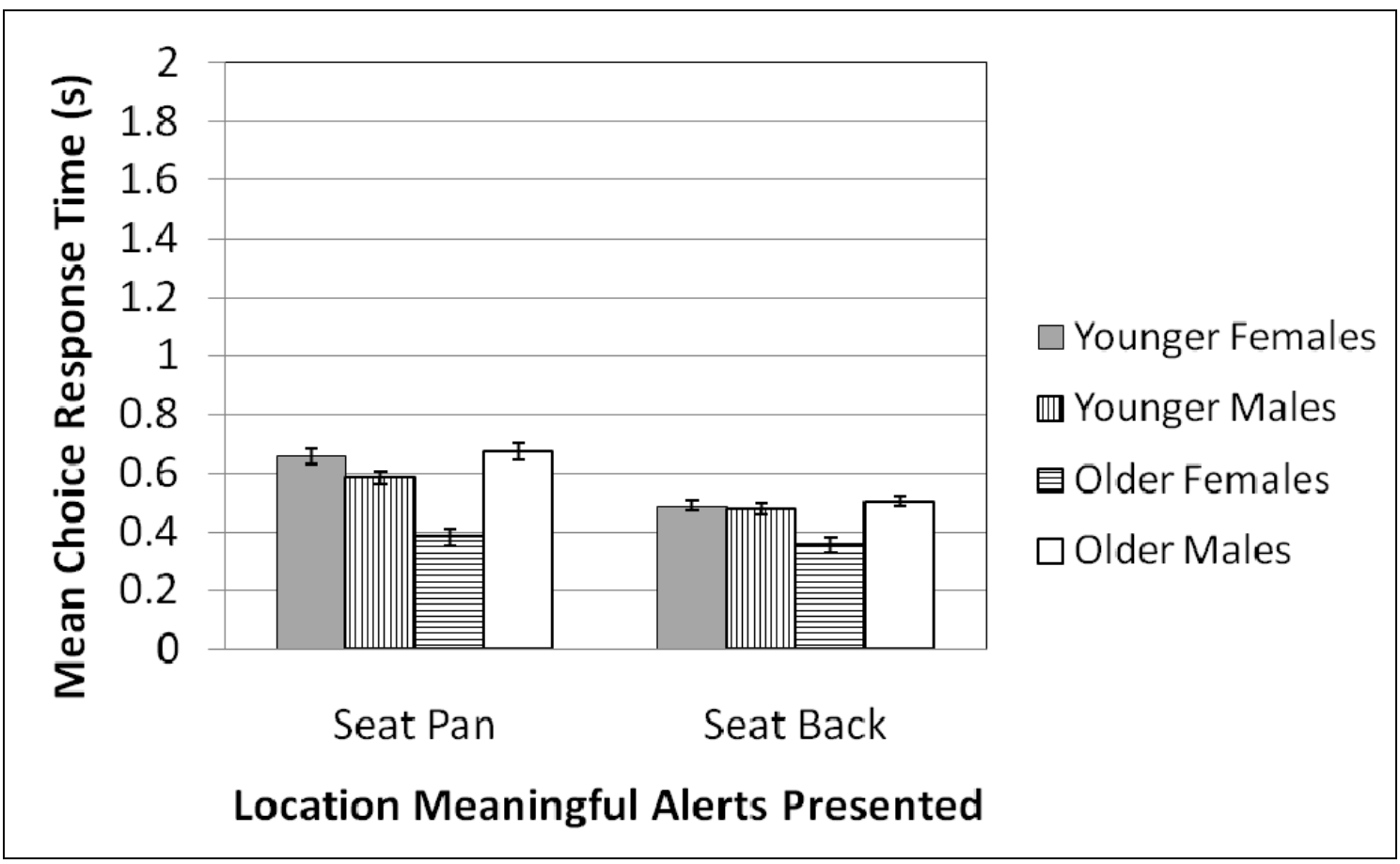

Figure 40. Drivers' $90^{\text {th }}$ percentile practical manual response times to meaningful alerts.

\section{Theoretical Manual Response Time}

All response times observed when participants performed the correct theoretical manual response are analyzed in this section. Figure 41 shows the histogram of drivers' theoretical manual response times to the meaningful alerts when they were presented through the seat pan, as well as when they were presented through the seat back. 


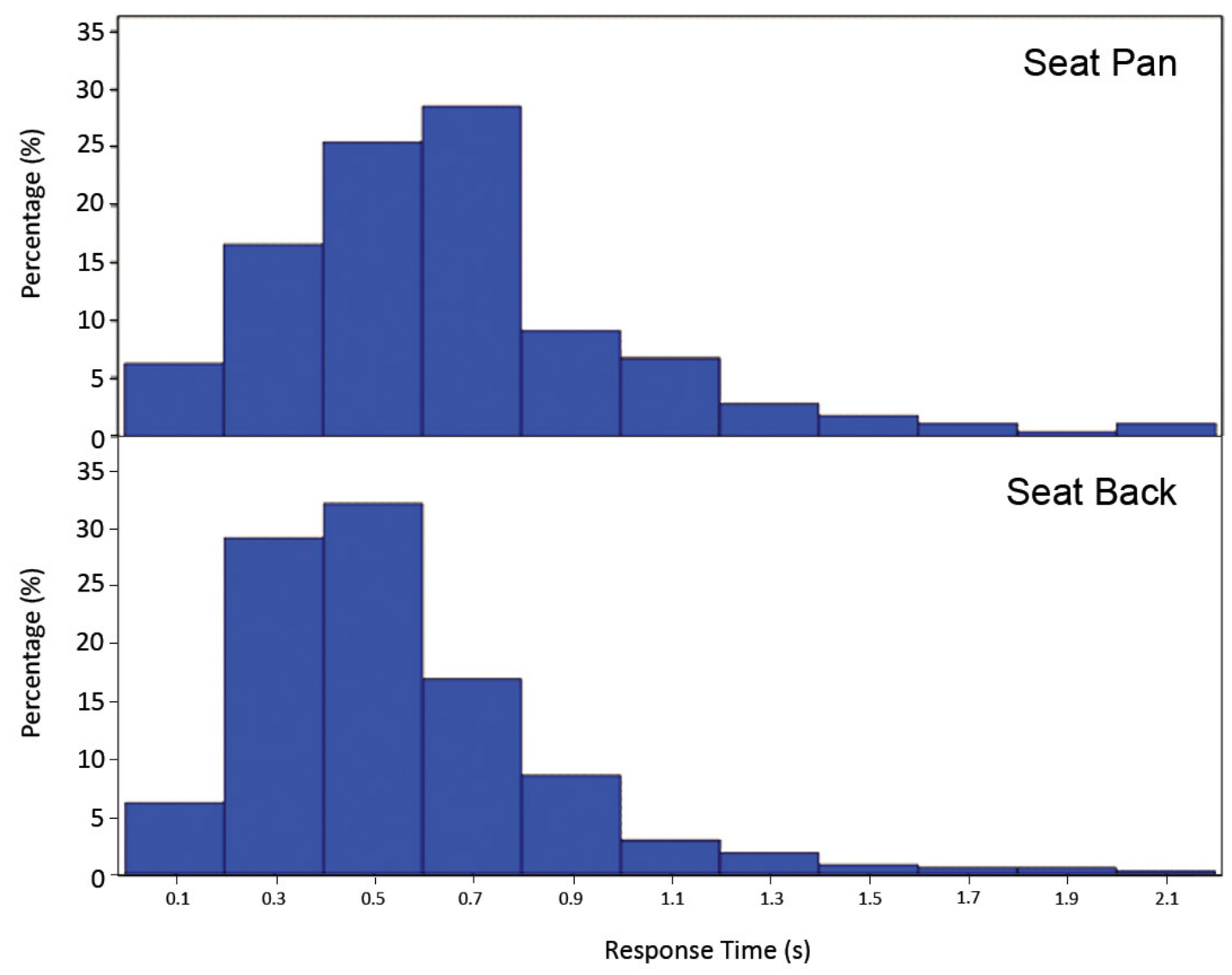

Figure 41. Histogram of drivers' theoretical manual response time to meaningful alerts presented through the seat pan, as well as through the seat back.

A 2 (Age) x 2 (Gender) x 2 (Side) x 2 (Location) mixed factors ANOVA was performed on the response time dependent variable. The location of the meaningful alerts was found to be a significant main effect $(\mathrm{F}(1,20)=26.23, p<0.0001)$. Drivers' mean response time to the meaningful alerts presented through the seat pan was $0.663 \mathrm{~s}$ (s.e. $=$ $0.021 \mathrm{~s}$ ), while their mean response time to meaningful alerts presented through the seat back was $0.522 \mathrm{~s}$ (s.e. $=0.015 \mathrm{~s})$. The side the alert was presented on was also found to significantly affect manual response time $(\mathrm{F}(1,20)=5.21, p=0.0335)$. Drivers' mean response time to left $\mathrm{LCW}$ alerts was $0.574 \mathrm{~s}$ (s.e. $=0.018 \mathrm{~s})$, while their mean response time to right $\mathrm{LCW}$ alerts was $0.611 \mathrm{~s}($ s.e. $=0.019 \mathrm{~s})$. A significant Age $\mathrm{x}$ Gender $\mathrm{x}$ Location interaction was also found $(\mathrm{F}(1,20)=4.85, p=0.0396)$. A Tukey-Kramer multiple comparisons test revealed that older male drivers' mean response time to meaningful alerts presented through the seat pan was significantly different from their mean response time to meaningful alerts presented through the seat back $(0.752 \mathrm{~s}$ (s.e. $=$ $0.037 \mathrm{~s}$ ) versus $0.560 \mathrm{~s}$ (s.e. $=0.024 \mathrm{~s}$ ), respectively. $p=0.027$ ). Similarly, younger female drivers' mean response time to meaningful alerts presented through the seat pan was significantly different from their mean response time to meaningful alerts presented through the seat back $(0.718 \mathrm{~s}(\mathrm{~s} . \mathrm{e} .=0.034 \mathrm{~s})$ versus $0.529 \mathrm{~s}(\mathrm{~s} . \mathrm{e} .=0.021 \mathrm{~s})$, respectively. 
$p=0.0264)$. These results are shown in Figure 42.

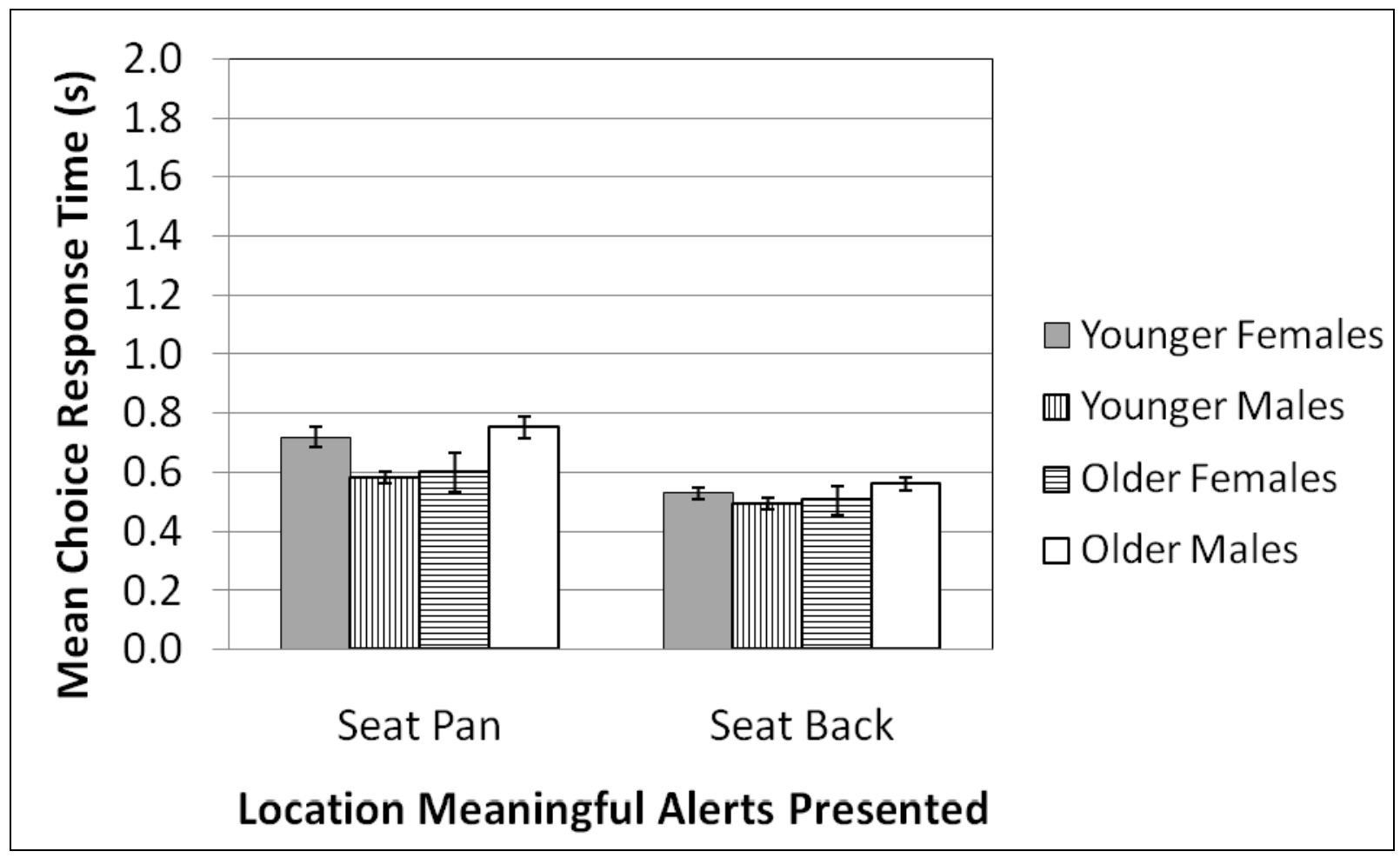

Figure 42. Drivers' mean theoretical manual response times to meaningful alerts.

The quantiles for drivers' theoretical manual response times to meaningful alerts presented through the seat pan and seat back are shown in Table 14. Figure 43 presents drivers' $90^{\text {th }}$ percentile response times broken down by their age and gender.

Table 14. Quantiles of Drivers' Theoretical Manual Response Times

\begin{tabular}{|c|c|c|}
\hline Quantile & Seat Pan (s) & Seat Back (s) \\
\hline $100 \%$ Max & 2.100 & 2.000 \\
\hline $99 \%$ & 2.067 & 1.567 \\
\hline $95 \%$ & 1.333 & 1.000 \\
\hline $90 \%$ & 1.100 & 0.833 \\
\hline $75 \%$ Q3 & 0.800 & 0.633 \\
\hline $50 \%$ Median & 0.633 & 0.467 \\
\hline $25 \%$ Q1 & 0.433 & 0.367 \\
\hline $10 \%$ & 0.300 & 0.267 \\
\hline $5 \%$ & 0.200 & 0.200 \\
\hline $1 \%$ & 0.033 & 0.067 \\
\hline $0 \%$ Min & 0.033 & 0.033 \\
\hline
\end{tabular}




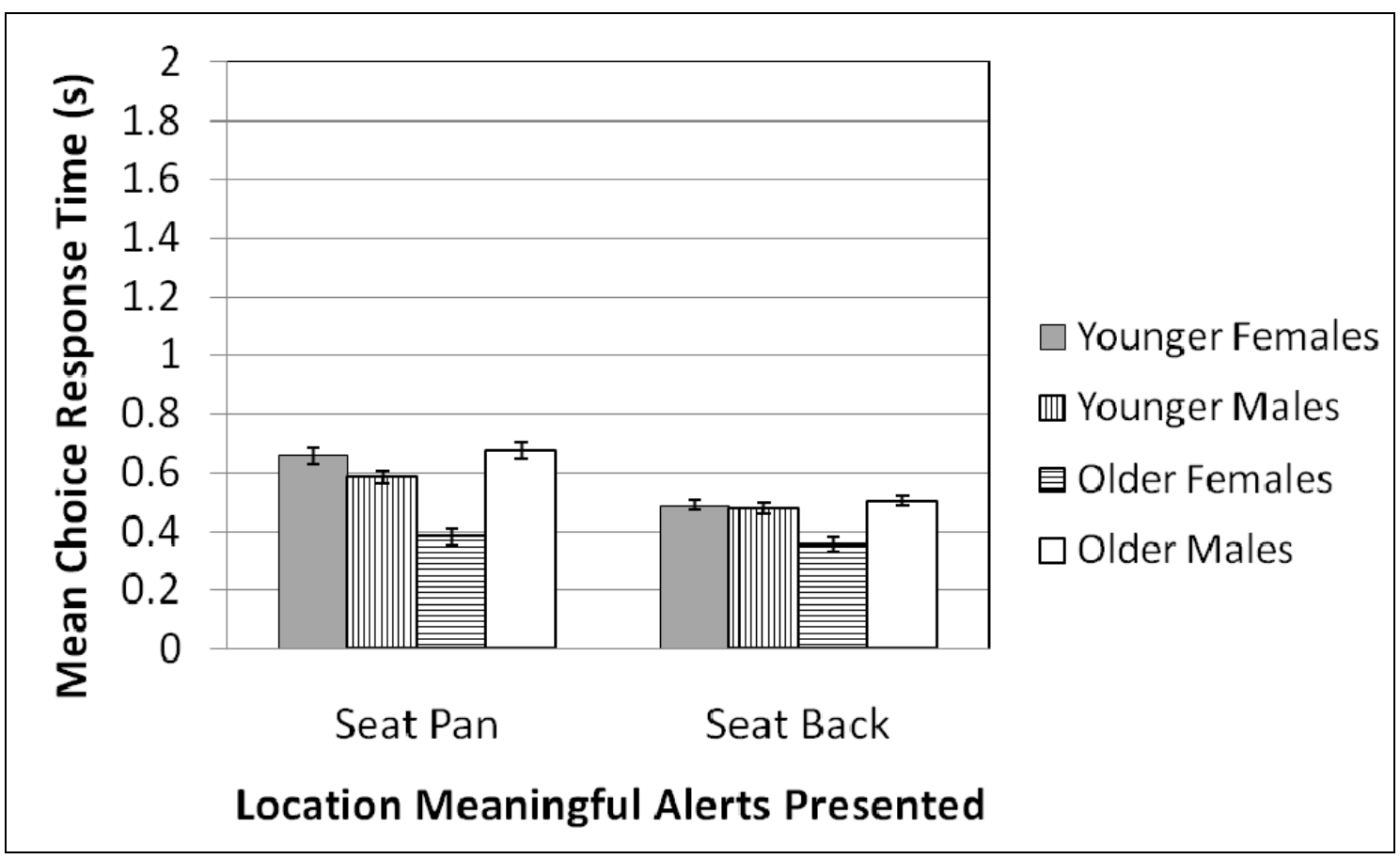

Figure 43. Drivers' $90^{\text {th }}$ percentile theoretical manual response times to meaningful alerts.

\section{Speed Accuracy Tradeoff Inspection}

By plotting drivers' mean manual response times to meaningful alerts against their mean manual response accuracy, an idea of whether drivers traded off making a correct response for quicker response times can be attained. Figure 44 below shows that older males as well as younger females were able to respond faster when the meaningful alerts were presented in a different location from the uninformative alerts while keeping their response accuracy consistently high. Younger males were observed to somewhat shorten their manual response times as well as improve their response accuracy when the meaningful alerts were presented through different locations. Older females were observed to improve their manual response accuracy, but did not shorten their response times. It can be seen that none of the drivers traded accuracy off for faster response times. In fact, all drivers were observed to shift their performance from the bottom right of the graph to the top left of the graph. This suggests that drivers were more efficient in processing the meaningful haptic seat alerts when they were presented through a different location than the uninformative alerts compared to when they were both presented through the same location in driver seat. 


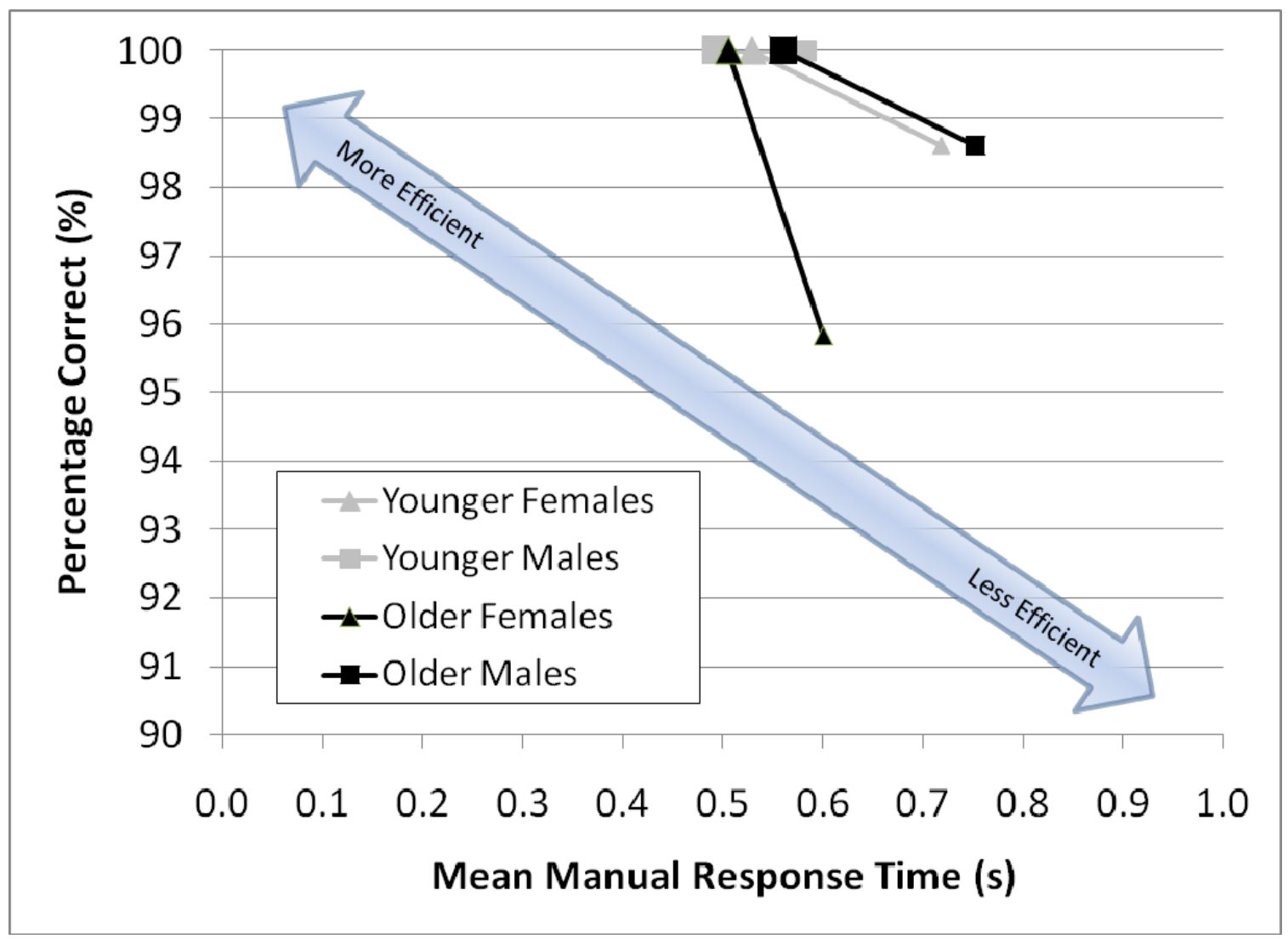

Figure 44. Speed-accuracy tradeoff inspection. The smaller symbols represent the speed-accuracy data points observed when the alerts were presented through the seat pan, while the larger symbols represent the speed-accuracy data points observed when the alerts were presented through the seat back.

\section{Driver Ratings}

This section analyzes drivers' perceptions of the alert identification task's difficulty. Drivers' ratings of task difficulty were measured through six questions which are presented below. The interested reader is referred to Appendix $\mathrm{K}$ for the results of driver's opinions regarding the alert's design.

\section{Question 1: Please rate how easy it was to distinguish meaningful vibration alerts from those you were not supposed to respond to.}

A 2 (Age) x 2 (Gender) x 2 (Location) mixed factors ANOVA was performed using drivers' ratings of how easy it was to distinguish meaningful alerts from uninformative alerts as the dependent variable. The location of the alerts was found to be a significant main effect $(\mathrm{F}(1,20)=13.75, p=0.0014)$. On average, drivers were neutral when asked to rate how easy it was to distinguish meaningful alerts from those they were not supposed to respond to when both alert types were presented through the seat pan (mean response $=4.44$, s.e. $=0.27$ ). In contrast, drivers found the task to be easy when the meaningful alerts were presented through the seat back and the uninformative alerts were 
presented through the seat pan (mean response $=5.46$, s.e. $=0.23$ ). Figure 45 breaks drivers' mean ratings down by age and gender. All drivers were observed to ameliorate their rating of task difficulty when the alerts were physically separated.

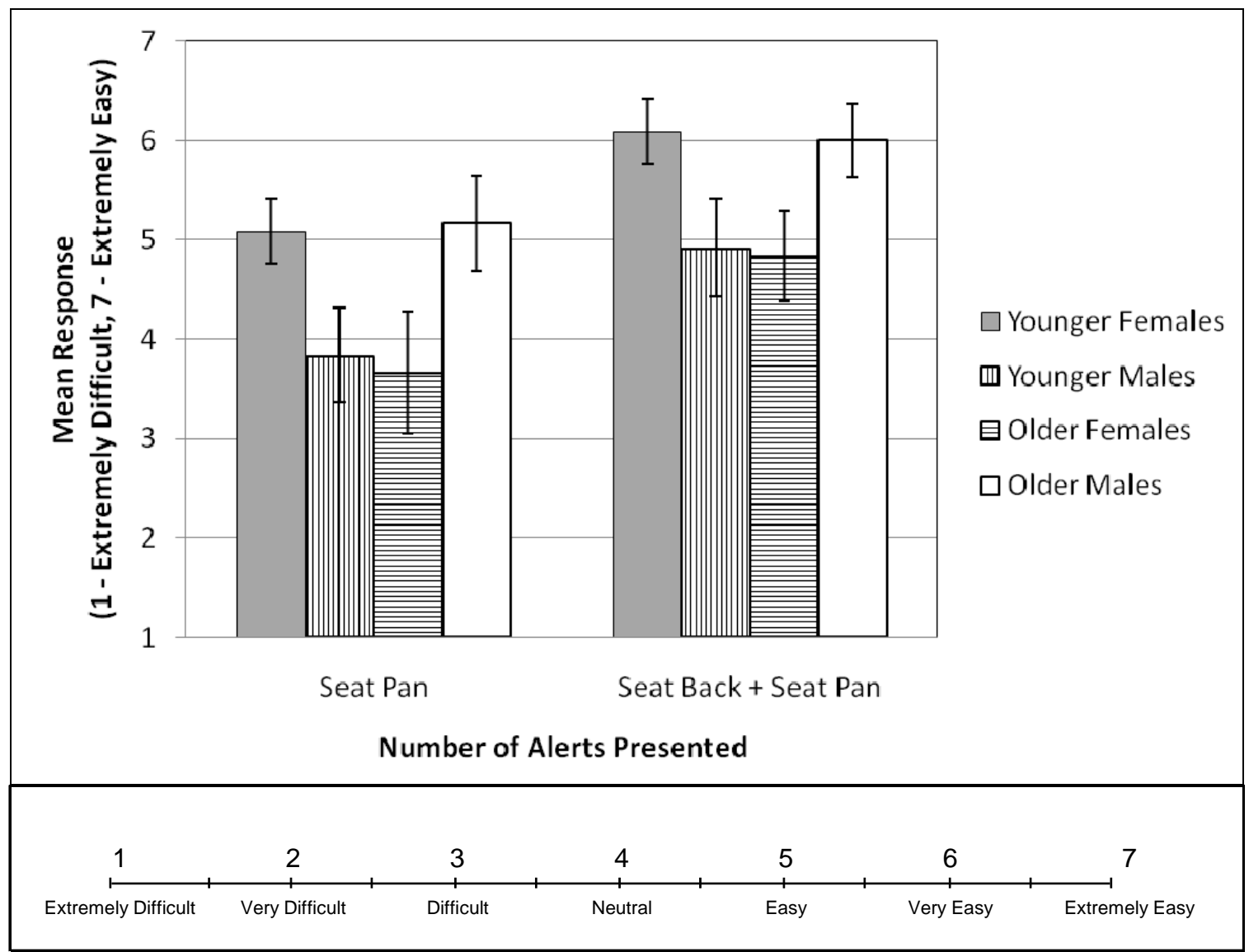

Figure 45. Drivers' mean ratings of how easy it is to distinguish meaningful vibration alerts from those that they are not supposed to respond to.

\section{Question 2: Please rate how distinct the vibration alerts were from each other.}

A 2 (Age) x 2 (Gender) x 2 (Location) mixed factors ANOVA was performed using drivers' ratings of how distinct the alerts were from each other as the dependent variable. The location of the alerts was found to be a significant main effect $(\mathrm{F}(1,20)=31.38, p<$ $0.0001)$. On average, drivers found the alerts to be well-defined when they were all presented through the seat pan (mean response $=4.67$, s.e. $=0.25$ ). In contrast, drivers found the vibration alerts to be very well-defined when the meaningful alerts were presented through the seat back and the uninformative alerts were presented through the seat pan (mean response $=6.04$, s.e. $=0.17$ ). Figure 46 breaks drivers' mean ratings down by age and gender. All drivers were observed to increase their rating of alert distinctiveness when the alerts were physically separated. 


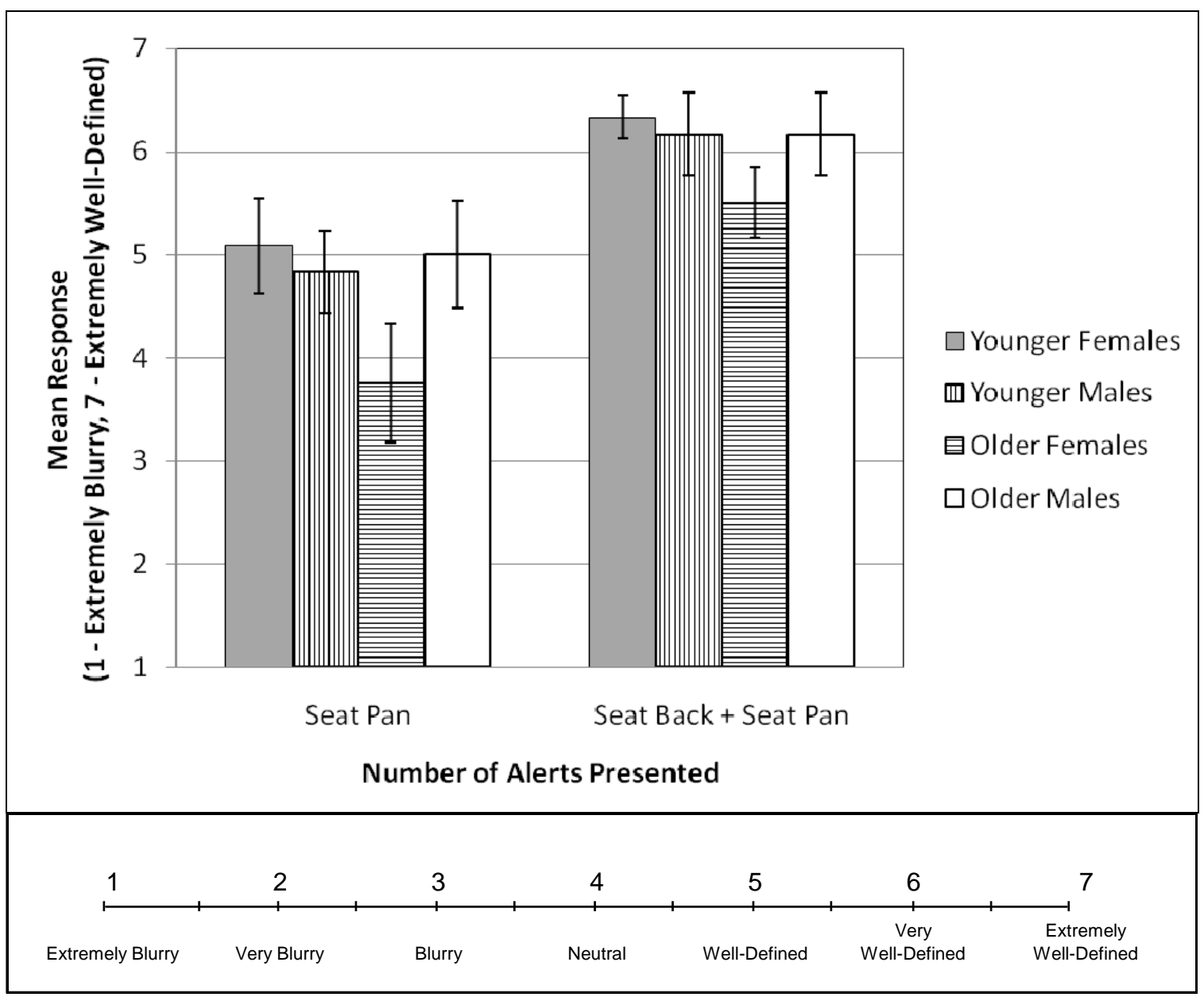

Figure 46. Drivers' ratings of how distinct the vibration alerts were from each other.

Question 3: Please rate how difficult it was to perform the task in Experiment 2.

A 2 (Age) x 2 (Gender) x 2 (Location) mixed factors ANOVA was performed using drivers' ratings of how easy it was to perform the task in Experiment 2 as the dependent variable. The location of the alerts was found to be a significant main effect $(\mathrm{F}(1,20)=$ $12.89, p=0.0018$ ). On average, drivers were neutral when asked to rate the difficulty of the overall task when the alerts were presented through the seat pan (mean response $=$ 4.50 , s.e. $=0.27$ ). In contrast, drivers found the overall task to be easy when the meaningful alerts were presented through the seat back and the uninformative alerts were presented through the seat pan (mean response $=5.50$, s.e. $=0.22)$. A significant Age $x$ Gender interaction was also found $(\mathrm{F}(1,20)=11.54, p=0.0029)$. Older female's mean rating of 4.25 (s.e. $=0.36)$ was significantly different from younger female's mean rating of 5.67 (s.e. $=0.36)$ (Adjusted $p=0.0483$ ). Figure 47 breaks drivers' mean ratings down by age and gender. All drivers were observed to ameliorate their rating of task difficulty when the alerts were physically separated. 


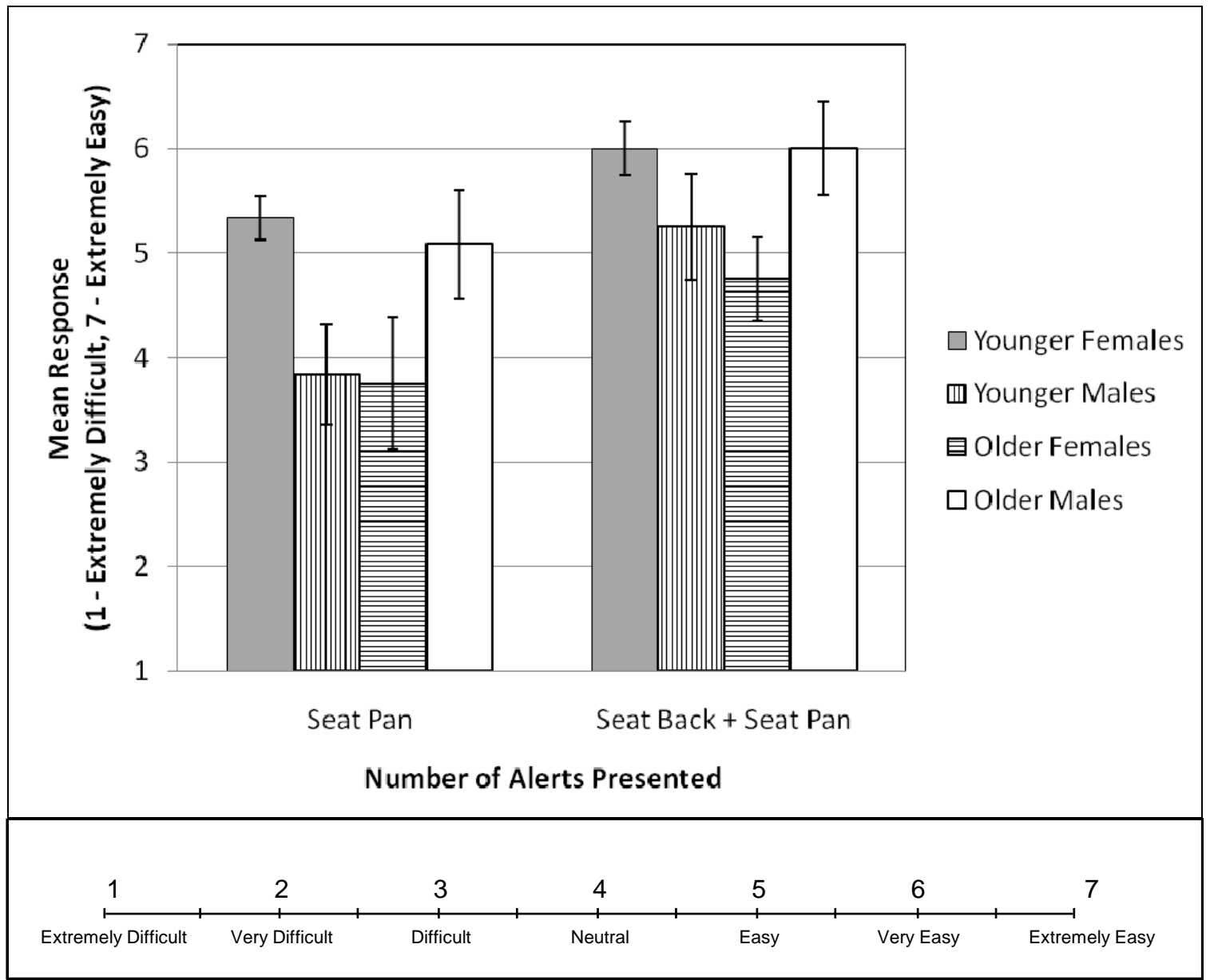

Figure 47. Drivers' mean ratings of how easy it is to perform the task in Experiment 2.

\section{DISCUSSION}

This experiment investigated the changes in driver performance that arise from presenting meaningful alerts through a physically separate haptic seat location than uninformative alerts compared to when both alerts are presented through the same physical location. The fundamental difference between these two arrangements centers on designing redundancy into the transfer of information. Two dimensions were used to convey information, the alert pattern as well as the location of the vibration in the driver seat. In the research field of multidimensional judgment, these two dimensions are termed "separable" because the levels of one dimension can be specified without specifying the levels of the other dimension (Wickens \& Hollands, 1999). When meaningful and uninformative alerts are presented through the same location, drivers can only rely on the vibrotactile pattern to distinguish the two alerts. The gray vertical line in Figure 48a shows the decision criterion set by a driver determining whether the perceived alert possessed a five-pulse pattern or a dash pattern. Here, the concentric circles are used to represent the three-dimensional bell-shaped probability distributions that the alert has a pulse or dash pattern. The area in the circle that lies on the other side of the 
decision criteria line represents the probability that a driver "misses" the alert (i.e., classifies it as having the wrong pattern).

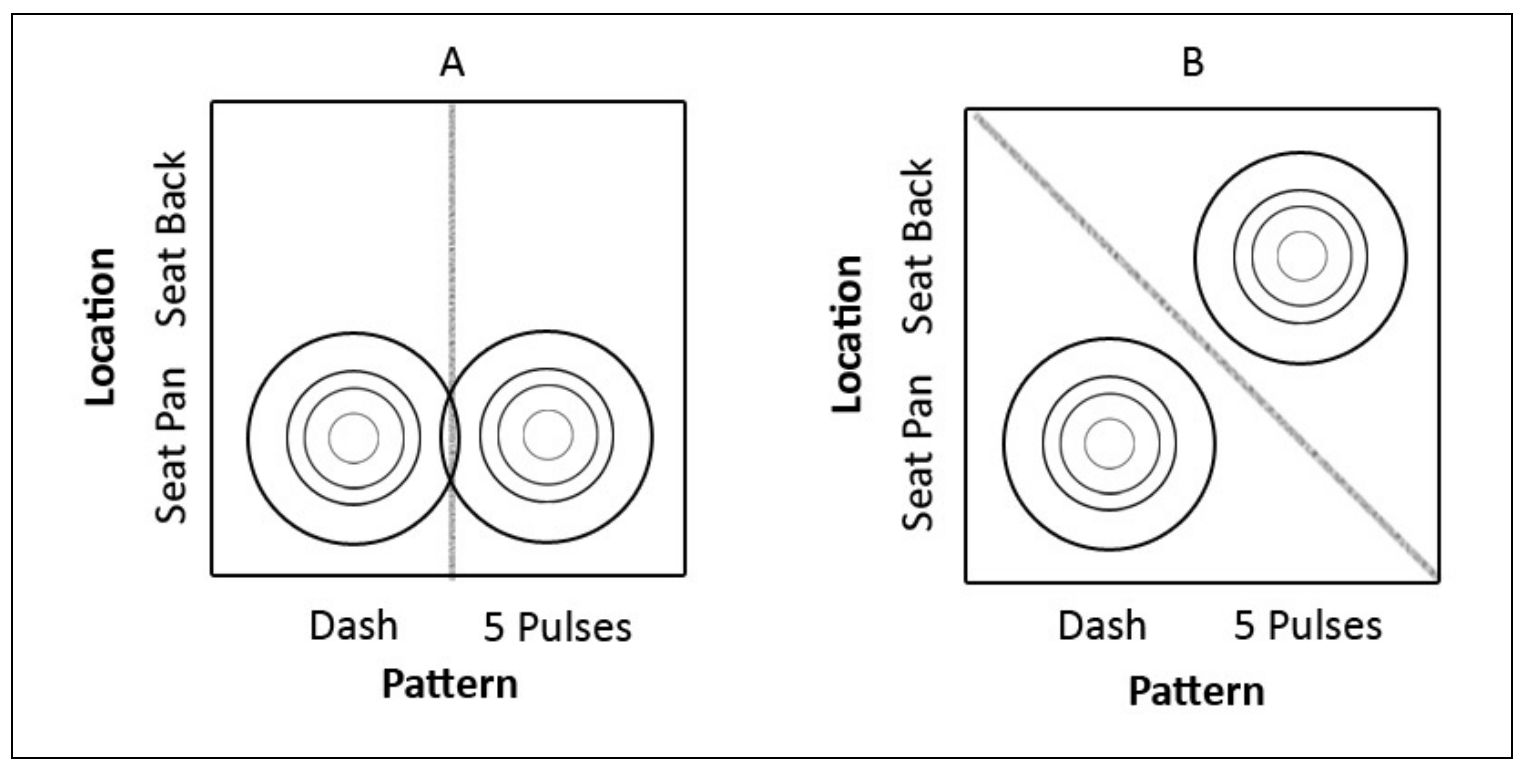

Figure 48. Representation of drivers' judgment of vibrotactile stimuli according to a) one dimension, and b) two correlated separable dimensions. Adapted from Wickens \& Hollands (1999).

In contrast, when meaningful alerts are presented in a different location from the uninformative alerts, drivers are able identify meaningful alerts using both the vibrotactile pattern as well as the location of the vibrations. These two dimensions are correlated such that the meaningful alerts are always presented through a different seat location than the uninformative alerts and they always consist of the pulse pattern while the uninformative alerts consist of the dash pattern. The decision criteria thus becomes a diagonal gray line that splits the two probability distributions shown in Figure 48b. It can be seen that the distance from the diagonal line to the center of either circle is lengthened, which represents a reduction in the probability of a miss (Wickens \& Hollands, 1999). Correlating the two dimensions serves to secure the information transmitted to the drivers, as exemplified with the improved accuracy scores and reduced response times.

The speed-accuracy tradeoff investigation performed provides an indication of whether drivers were more efficient at processing the meaningful alerts according to the two correlated separable dimensions of vibrotactile pattern and location compared to when they just had the one dimension of vibrotactile pattern. The general shift towards the upper left of the graph shown in Figure 44 was evidence of an improvement in processing efficiency. Older females improved their mean response time by $0.094 \mathrm{~s}$. Older males improved their mean response time by $0.192 \mathrm{~s}$. Younger females improved their mean response time by $0.189 \mathrm{~s}$. Younger males improved their mean response time by $0.089 \mathrm{~s}$.

Older females were observed to struggle significantly more with the experimental task compared to the other drivers. This is clearly shown in the speed-accuracy tradeoff 
analysis. Although their performance improved when the alerts were presented through different locations, older female drivers were still less efficient at processing information than the other drivers. It is not quite clear why this occurred and it is perhaps an anomaly. However, older females did indicate that the meaningful alerts were not welldefined relative to the uninformative alerts when they were both presented through the seat pan, while the other drivers rated these alerts to be well-defined. It is possible that older females' perception of the vibrotactile patterns may not have been as sensitive as the other drivers, leading to their poorer performance.

Overall, statistically significant improvements in manual response accuracy and time were found when the meaningful alerts were presented through a different location than the uninformative alerts. Drivers' also indicated that it was easier to distinguish the meaningful alerts from the uninformative alerts when they were presented through a different location. These results suggest that there is a benefit to presenting haptic seat alerts through unique areas in the driver seat. This is likely only true if the areas are distanced from each other. Spacing the haptic seat alerts apart is believed to prevent confusion regarding which area is vibrating. For instance, Fitch, Kiefer, Kleiner, et al. (2007) found that drivers mistook haptic alerts presented through unique locations in the seat for each other because the areas that vibrated were contiguous.

Experiment 1 showed that performance decrements occurred when the amount of information conveyed by the three-alert approach increased to that conveyed by the seven-alert approach. At the same time, the seven-alert approach was the only approach that did not use unique tactors for each of the alerts. As a result, it is also possible that the observed performance decrements occurred owing to common tactors being used by the alerts. Experiment 2 controlled the amount of information presented to drivers, but varied the location of the alerts. It was found that presenting alerts through unique tactors benefited drivers' response performance. The results from these two experiments suggest that a seven-alert approach that uses unique tactors is worth investigating.

The findings from Experiment 2 should not deemphasize drivers' performance when all alerts were presented through the seat pan. In this setup, drivers were still quite efficient at perceiving, processing, and responding to the meaningful alerts. Drivers performed the correct manual response 86 percent of the time (older females scored the worst with a manual response accuracy of 71 percent). Drivers' average mean response time to the alerts was 0.666 seconds. As a result, a seat-pan-only haptic seat design is still worth considering for further testing

In analyzing lane change crashes and near crashes, Fitch et al. (2009) found that an average of $1.5 \mathrm{~s}$ elapsed from a vehicle initiating a planned lane-change to it crossing into the adjacent left lane. They also found that the lane change events in this scenario occurred on average in $2.3 \mathrm{~s}$. If LCW alerts were to be presented $0.5 \mathrm{~s}$ into the execution of these lane changes, drivers would have $1.134 \mathrm{~s}$ to make an evasive action when the alerts were presented through the seat pan, and they would have $0.126 \mathrm{~s}$ to make an evasive action when the alerts were presented through the seat back. It is the authors' opinion that adequate evasive action could be performed in both situations. 


\section{CHAPTER 5. EXPERIMENT 3: EFFECTS OF A HAPTIC SEAT ALERT ON DRIVER COLLISION AVOIDANCE BEHAVIOR}

\section{PURPOSE}

The previous two experiments investigated drivers' ability to comprehend various haptic seat alerts and execute specific driving maneuvers in response. Although drivers were informed that the alerts represented specific collision warnings, the alerts were not generated in response to actual crash threats. Furthermore, since many alerts were presented over the course of each experiment, drivers may have developed an expectation for the alerts. Their response times may have been faster than if the alerts had been unexpectedly presented as a result. The purpose of this experiment was to address these potential shortcomings and investigate the haptic driver seat's ability to alert distracted drivers to an actual forward threat. There are no known studies that investigate the use of a haptic driver seat to warn drivers of an actual crash threat.

\section{METHOD}

The method used in Experiment 3 is presented in this section. Since the selected participants, test vehicle, test track, DAS, as well as the video recording hardware and software were the same as those used in Experiments 1 and 2, these aspects of the study are not described. However, since the haptic driver seat design and experimental procedure were changed, they are described below. The device used to present a crash threat to drivers is also described.

\section{Apparatus}

\section{Haptic Driver Seat}

The haptic driver seat only presented the FCW alert in this experiment. The pattern used was the same as that used in Experiment 1 (Table 15). The total amount of time the tactors vibrated was set to be one second.

Table 15. Experiment 3 Vibrotactile Seat Alert Pattern

\begin{tabular}{|l|l|}
\hline \multicolumn{1}{|c|}{ Alert } & \multicolumn{1}{c|}{ Haptic Pattern } \\
\hline FCW Alert & $\begin{array}{l}\text { Front-left and front-right tactors pulse five times }(200 \mathrm{~ms} \\
\text { on, 50 ms off) }\end{array}$ \\
\hline
\end{tabular}

\section{Clarion Multimedia Center}

A Clarion touch-screen multimedia center was used for the distraction task (Figure 49). The device allowed drivers to select music from FM/AM radio, Sirius XM satellite radio, $\mathrm{CD} / \mathrm{MP} 3 \mathrm{~s}$, and DVDs by touching icons presented on the display. The touch-screen display was concealed during Experiments 1 and 2. The experimenter opened the screen prior to beginning Experiment 3. It should be noted that the display never played music during this Experiment. Rather, drivers were asked to navigate a hierarchical menu. 

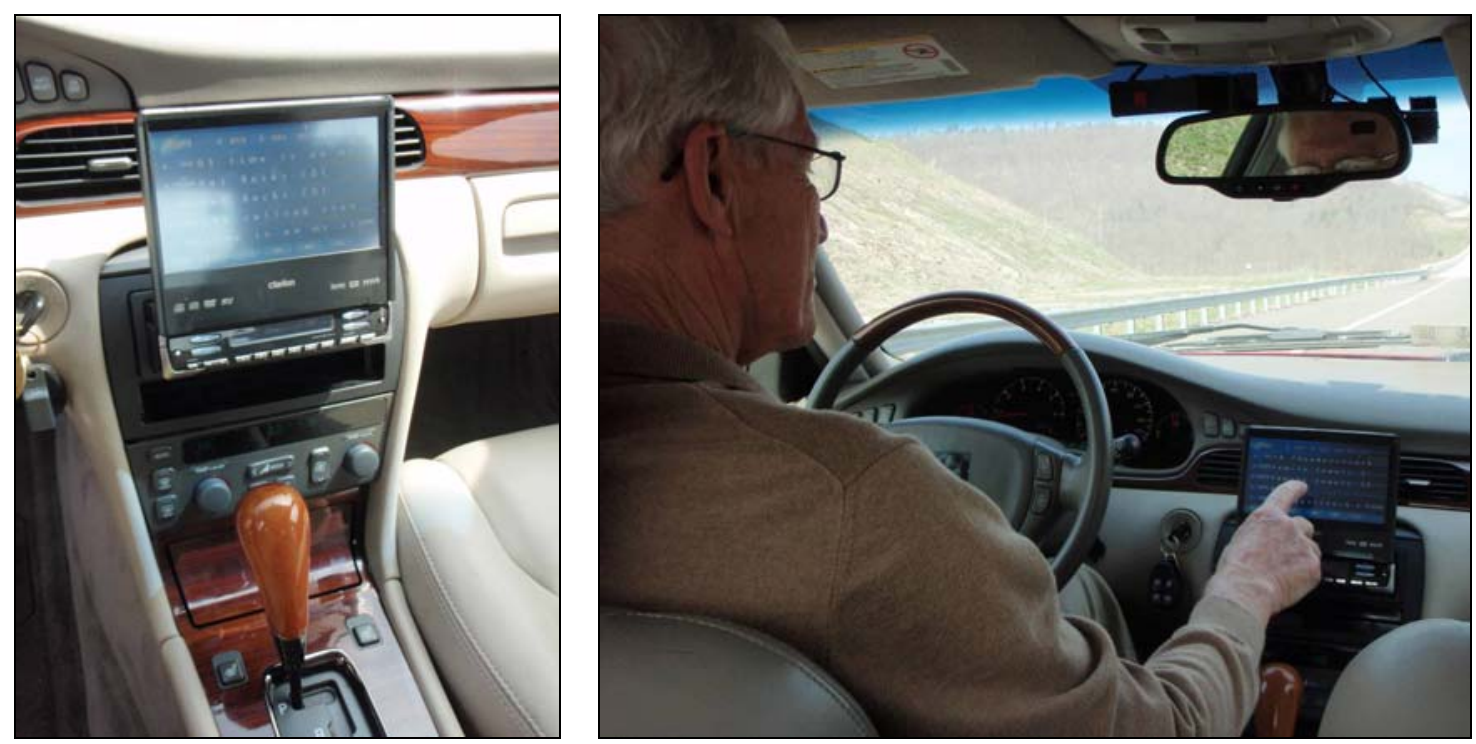

Figure 49. Clarion touch-screen multimedia center.

\section{The Inflatable Barricade}

The inflatable barricade (Figure 50) was developed in a previous braking study performed at VTTI (Fitch, et al., In Press). It consisted of eight neon-green nylon cylinders that were concealed in holes bored into the Smart Road. The holes were covered by plastic caps so drivers would not notice the neon-green material as they drove over them. A command to inflate the cylinders was wirelessly sent from the vehicle by the experimenter to the Smart Road. An air compressor hidden in a ditch adjacent to the road inflated the cylinders. The cylinders stood 4 feet tall for ten seconds when inflated and were highly visible. The eight cylinders were four feet apart and spanned both lanes of the Smart Road as well as the road's shoulder. Drivers did not have room to swerve around them as a result. The nylon cylinders were designed to allow a test vehicle to repeatedly and safely drive over them in case they were not seen. Tests in which the test vehicle collided with nylon cylinders at $105 \mathrm{~km} / \mathrm{h}(65 \mathrm{mph})$ showed that no damage occurred to the test vehicle; the driver did not feel a collision occurred. The area where the braking event took place was free of protruding obstacles. 


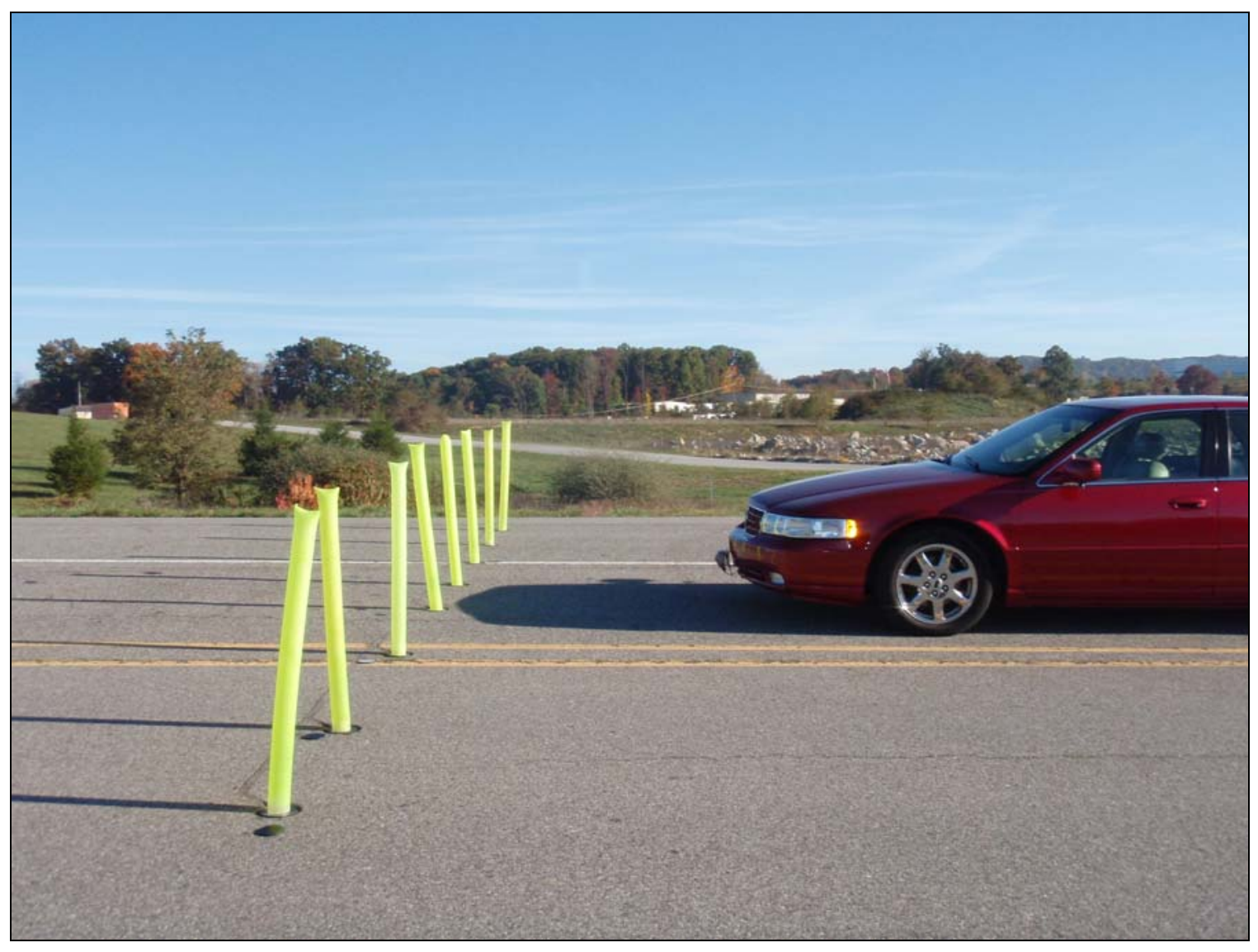

Figure 50. Eight inflatable nylon cylinders that span the Smart Road lanes and right shoulder.

\section{Differential Global Positioning System (DGPS)}

A Differential Global Positioning System (DGPS) was used to precisely locate the test vehicle on the Smart Road. Differential GPS corrections were broadcast from the research building to the road. The test vehicle was equipped with a portable GPS unit that, combined with the differential GPS corrections, allowed for extremely accurate (on the order of $\pm 1.5 \mathrm{~cm}$ ) vehicle positioning (Figure 51). A precise measure of the vehicle's Time-to-Collision (TTC) to the inflatable barricade, as well as final distance from the barricade, was thus available. 


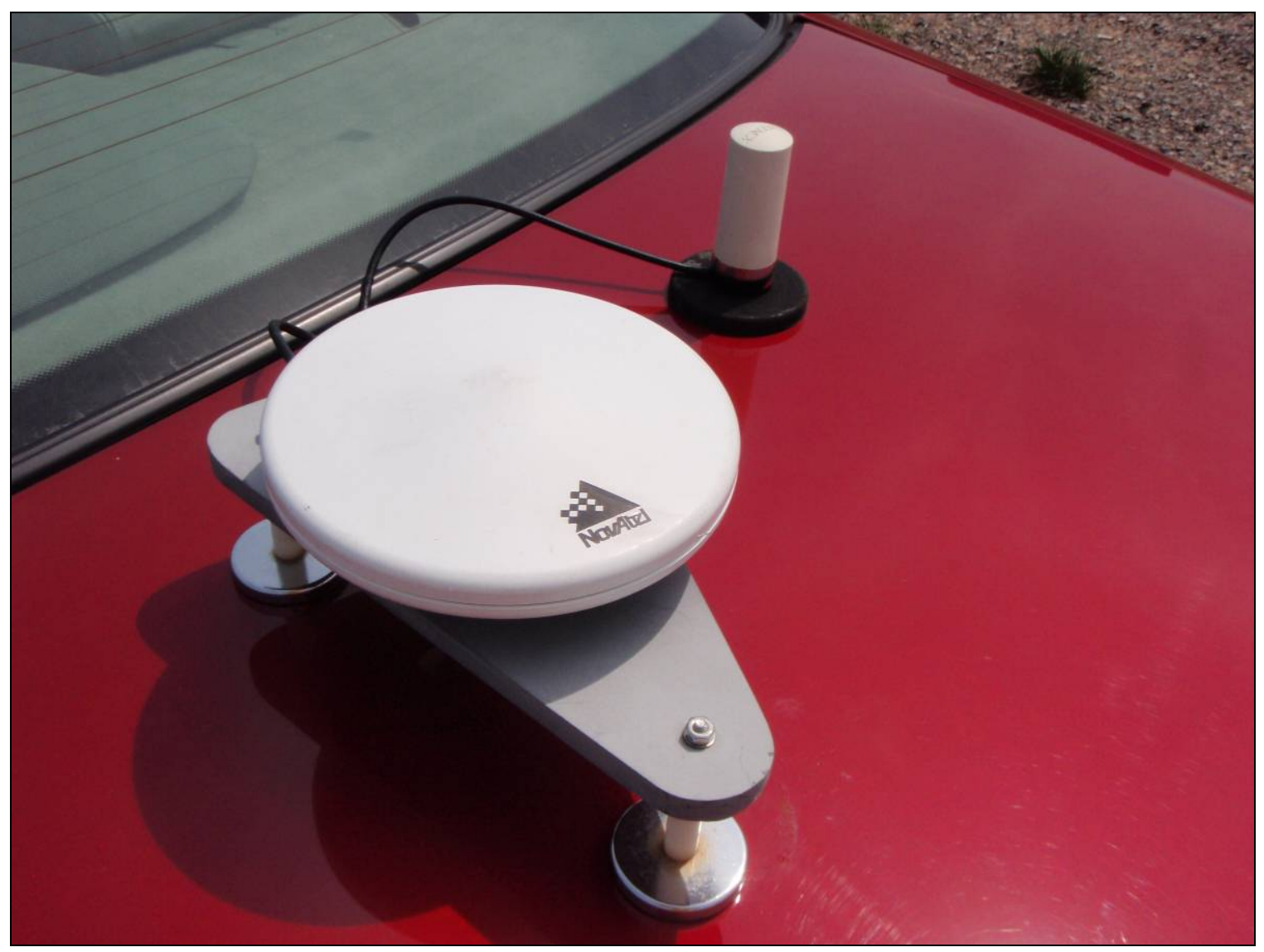

Figure 51. Antenna mounted to trunk of test vehicle that was used for the Differential Global Position System (DGPS).

\section{Procedure}

Once participants completed Experiment 1 and 2, the in-vehicle experimenter explained that the next part of the study involved operating the Clarion CD/MP3 player. Participants drove at $20 \mathrm{mph}$ up the right lane of the Smart Road. As participants drove up the road, the experimenter asked participants to look for a specific icon on the Clarion touch-screen display and press it when the command to do so was given. Participants were asked to perform the task as fast and as accurately as possible. They were also asked to not look at the display until the command to do so was given. Participants practiced this task once before driving the vehicle up the Smart Road. They then performed this task five times while they drove up the Smart Road. Each task involved one button press only. Over the course of the five trials, participants drilled down the Clarion's menu structure and back up to the root menu.

As the vehicle approached the inflatable barricade, the experimenter gave the command to look for a menu option that did not exist. As participants scanned the display for the non-existent option, the experimenter sent a command to the Smart Road to launch the inflatable barricade. The experimenter tried to consistently launch the barricade as the participants fixated on the display. This could be roughly identified by observing their 
head turning towards the display. Half of the participants received an FCW alert at the same time the command to inflate the barricade was given. The other half did not. After participants brought the vehicle to a stop, the experimenter explained the additional purpose of the study. It should be mentioned that participants signed an informed consent form that stated

"Some studies at VTTI involve an unanticipated event. You may or may not encounter such an event during this study. Please be aware that equipment failure, changes in the test track, stray or wild animals entering the road, and weather changes may require you to respond accordingly. The appropriate response may or may not involve rapid deceleration."

Participants were asked to complete a questionnaire regarding their experience with the unexpected braking event. Participants were then asked to drive back to the Virginia Tech Transportation Institute, where they were paid \$20/hour for their time.

\section{Independent Variables}

This experiment consists of a 2 (Age) x 2 (Gender) x 2 (Alert) between-subjects design.

\section{Age (Older, Younger) - Between-Subjects}

The same participants from Experiments 1 and 2 were used. Drivers were selected from two age groups: a younger age group consisting of drivers between the ages of 18 and 25 years old, as well as an older age group consisting of drivers that were above 65 years old. The age independent variable allowed younger and older drivers' ability to utilize the FCW alert to be investigated.

\section{Gender (Female, Male) - Between-Subjects}

Both female and male drivers were equally selected for this Experiment to investigate the presence of a gender effect. Gender was deemed an important factor to generalize the results to the driving population.

\section{Alert (On, Off) - Between-Subjects}

Half of the drivers received an FCW alert as the barricade inflated, while the other half did not. The effects of the FCW alert on driver's collision avoidance behavior to an unexpected crash threat was investigated.

\section{Dependent Variables}

The following measures were used to investigate drivers' collision avoidance behavior to an unexpected crash threat. 


\section{Collision with Barricade}

The number of collisions with the inflatable barricade was recorded. Drivers were only exposed to the barricade one time.

\section{Time from Inflatable Barricade Activation to Returning Eyes to the Forward Roadway}

The elapsed time from the barricade being inflated to drivers returning their gaze to the forward roadway was measured. This measure was performed using the recorded video footage of the driver's face. The exact time the barricade inflated was recorded by the DAS.

\section{Foot-Off Throttle Response Time}

The elapsed time from the barricade being inflated to drivers beginning to lift their foot up on the throttle was measured. Video footage of the driver's foot made this feasible. The point in time the driver began to lift up on the throttle, not the point where the driver's foot lifted off the gas pedal, was recorded.

\section{Foot-On Brake Response Time}

The elapsed time from the barricade being inflated to drivers pressing the brake pedal was measured. Again, this was possible using the video footage of drivers' feet.

\section{Number of Drivers that Pressed the Brake Pedal Prior to Returning their Gaze to the Forward Roadway}

The number of drivers that press the brake pedal prior to looking back up at the road was investigated. The effects of presenting the FCW alert on this behavior were explored.

\section{Remaining Distance to Barricade}

The remaining distance to the barricade was measured using DGPS.

\section{Peak Deceleration}

The peak deceleration drivers experienced was recorded using an accelerometer. This measure was used to characterize the braking maneuver performed.

\section{Driver Ratings}

Drivers' ratings of the unexpected braking event were collected through a questionnaire. Drivers that received the FCW alert were also asked to rate their experience with the alert. Appendix L presents the questions that were given to participants in the baseline condition, while Appendix M presents the questions that were given to participants who received the $\mathrm{FCW}$ alert. 


\section{Hypotheses}

Participants were involved in a distraction task when the barricade was inflated. It was hypothesized that participants receiving an FCW alert would have fewer collisions with the barricade than those that did not receive an alert. It was also hypothesized that drivers receiving an alert would return their gaze to the forward roadway sooner, lift their foot off the throttle sooner, and press the brake pedal sooner compared to drivers that did not receive an alert. Owing to their experience with the FCW alert in Experiment 1, the number of drivers that pressed the brake pedal prior to looking at the forward roadway was expected to be more for the group that received an alert compared to those drivers that did not receive an alert. These drivers were also expected to stop further away from the barricade than drivers that did not receive an alert since these drivers would likely travel a farther distance before realizing a barricade was present. It was also hypothesized that drivers receiving an FCW alert would have higher peak decelerations than drivers that did not receive an FCW alert.

\section{RESULTS}

Since the barricade was inflated at the experimenter's discretion, an investigation was performed to determine whether drivers who did not receive a haptic alert encountered the barricade sooner than the drivers that did receive a haptic alert. A 2 (Age) x 2 (Gender) x 2 (Alert) between-subjects ANOVA was performed on the Time-To-Collision (TTC) in which the barricade was inflated. A significant difference in the TTC values was not found. On average, the barricade was inflated with a TTC of $7.35 \mathrm{~s}$ (s.e. $=0.18$ s) when no FCW alert was provided, and a TTC of 7.49 s (s.e. $=0.25 \mathrm{~s}$ ) when an FCW alert was presented. As such, there is no evidence that the conditions under which the surprise braking event took place were unequal across the two groups.

\section{Collision with Barricade}

Two of the 24 drivers collided with the inflatable barricade. Both were older males. One driver was in the group that did not receive an FCW alert, while the other driver was in the group that received an FCW alert. The driver that received the FCW alert was observed to completely ignore it. This driver continued to perform the distraction task as he drove through the inflatable barricade.

Appendix N presents scatter plots of the variables analyzed in this study. Since the data points associated with the trials in which the drivers ran through the barricade were found to be outliers, these two drivers were removed from the rest of the analyses performed in this study.

\section{Time from Barricade Inflating to Driver Returning Eyes to the Forward Roadway}

A 2 (Age) x 2 (Gender) x 2 (Alert) between-subjects ANOVA was performed on the time drivers took to return their gaze to the forward roadway. It was found that drivers that received an FCW alert at the time the barricade was inflated returned their gaze to the forward roadway significantly faster than drivers that did not receive an alert $F(1,14)=$ 
$26.51, p=0.0001)$. Drivers that did not receive an FCW alert took 3.80 s (s.e. $=0.38$ s) to look at the forward roadway, while drivers that received an alert took $1.55 \mathrm{~s}$ ( $\mathrm{s} . \mathrm{e}=0.21$ s). Figure 52 presents drivers' mean time to return their eyes to the forward roadway according to their age and gender.

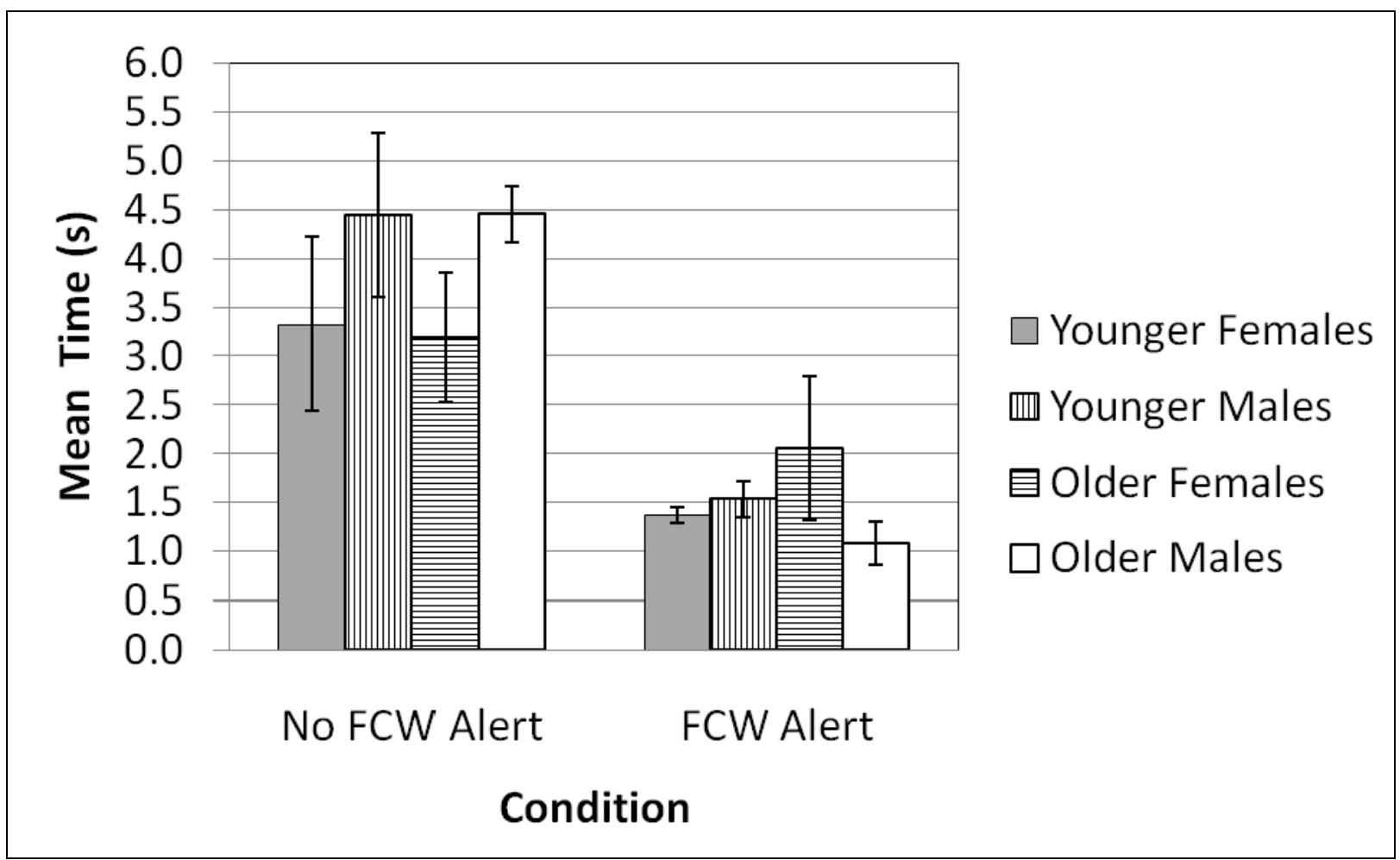

Figure 52. Drivers' mean time to return their gaze to the forward roadway broken down by age and gender.

\section{Foot-Off Throttle Response Time}

A 2 (Age) x 2 (Gender) x 2 (Alert) between-subjects ANOVA was performed on the elapsed time from the barricade being inflated to drivers beginning to remove their foot off the throttle. It was found that drivers that received an FCW alert at the time the barricade was inflated removed their foot from the throttle significantly sooner than drivers that did not receive an alert $\mathrm{F}(1,14)=15.96, p=0.0013)$. Drivers that did not receive an $\mathrm{FCW}$ alert took $3.76 \mathrm{~s}$ (s.e. $=0.48 \mathrm{~s}$ ) to remove their foot from the throttle, while drivers that received an alert took $1.55 \mathrm{~s}(\mathrm{~s} . \mathrm{e}=0.26 \mathrm{~s})$. Figure 53 presents drivers' mean time to take their foot off the throttle according to their age and gender. 


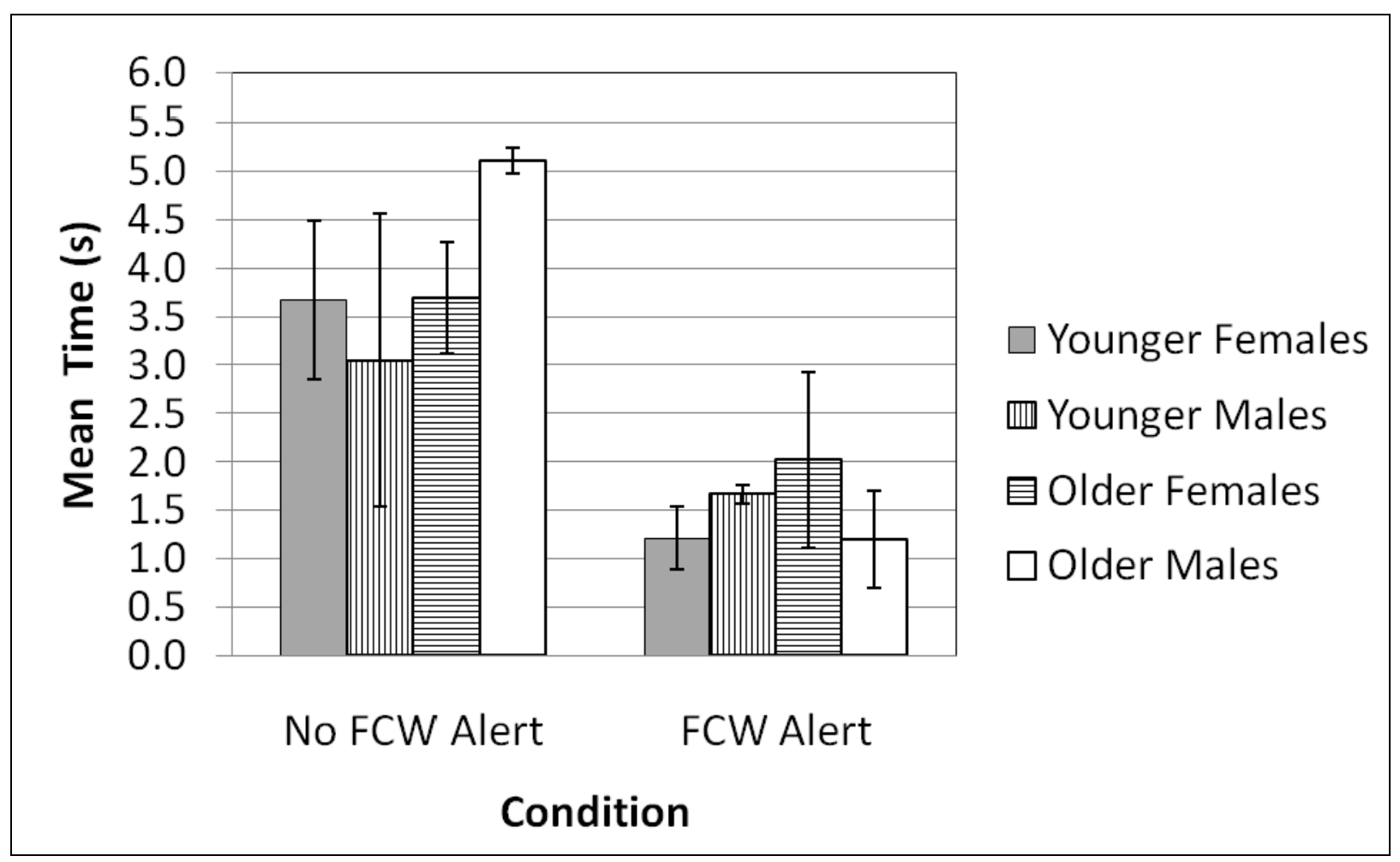

Figure 53. Drivers' mean time to remove their foot from the throttle broken down by age and gender.

\section{Foot-On Brake Response Time}

A 2 (Age) x 2 (Gender) x 2 (Alert) between-subjects ANOVA was performed on the elapsed time from the barricade being inflated to drivers pressing the brake pedal. It was found that drivers that received an FCW alert at the time the barricade inflated pressed the brake pedal significantly sooner than drivers that did not receive an alert $F(1,14)=$ $40.28, p<0.0001)$. Drivers that did not receive an FCW alert took $4.47 \mathrm{~s}($ s.e. $=0.31 \mathrm{~s})$ to press the brake pedal, while drivers that received an alert took $1.96 \mathrm{~s}$ ( $\mathrm{s} . \mathrm{e}=0.24 \mathrm{~s}$ ).

Figure 54 presents drivers' mean time to press the brake pedal according to their age and gender. 


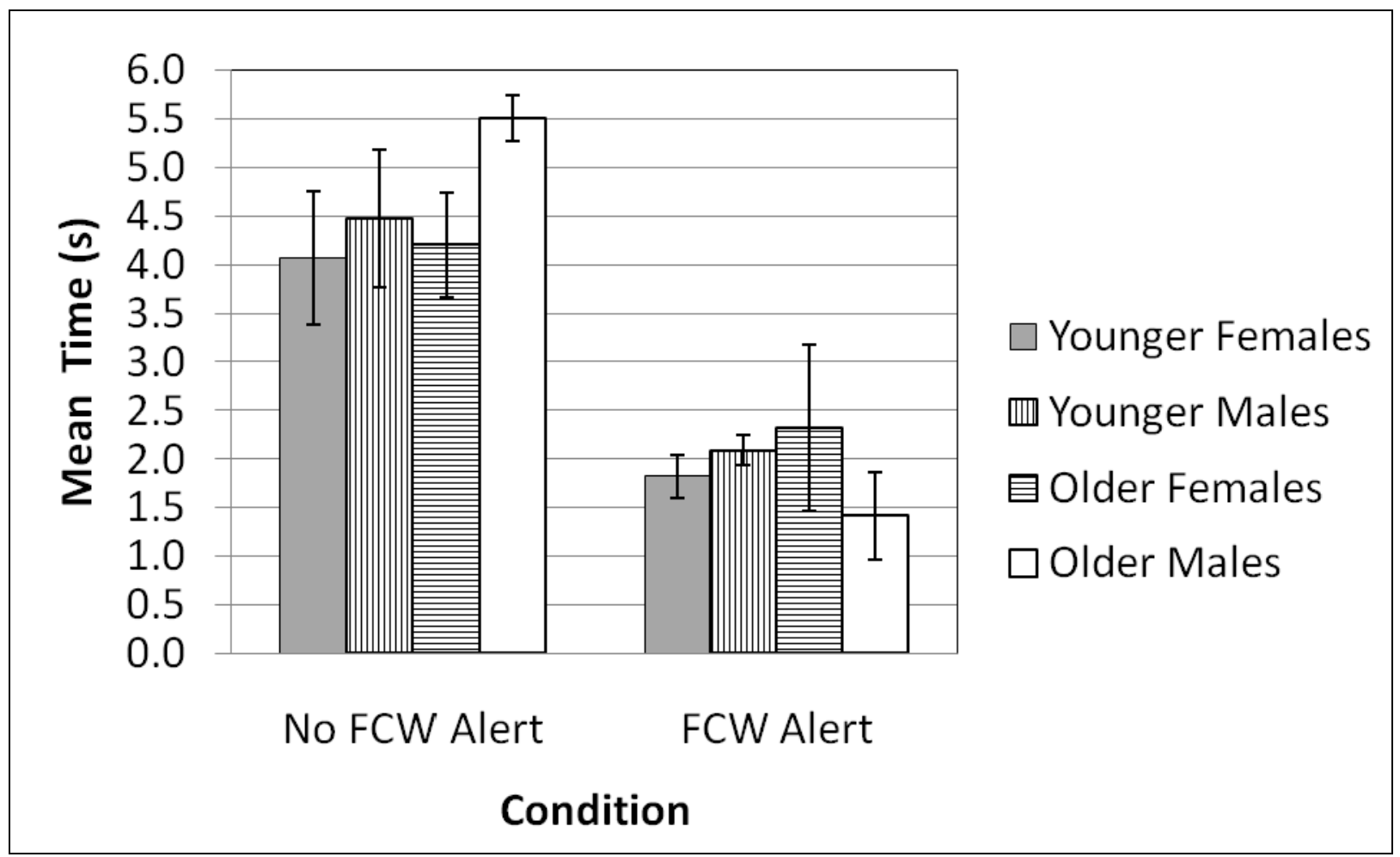

Figure 54. Drivers' mean time to press the brake pedal broken down by age and gender.

\section{Number of Drivers that Pressed the Brake Pedal Prior to Returning Gaze to the Forward Roadway}

Further analysis revealed that 6 of the 12 drivers that received an FCW alert removed their foot from the gas pedal prior to returning their gaze to the forward roadway. One driver even pressed the brake pedal prior to looking up at the road. In contrast, only one of the 12 drivers that did not receive an FCW alert removed their foot from the gas pedal prior to returning their gaze to the forward roadway. This driver also pressed the brake pedal prior to looking at the forward roadway. It should be noted that this driver was focused on the distraction task and had travelled a long distance without looking at the road. Pressing the brake pedal was likely a consequence of being unsure of where the vehicle was heading while looking down at the display. Perhaps the drivers that received the FCW alert developed an automatic response to slow the vehicle down upon receiving an FCW alert as a result of their trials in Experiment 1. It should be noted that a statistically significant difference between the number of drivers that removed their foot from the throttle prior to looking forward when receiving an FCW alert and those drivers that did not receive an FCW alert was not found (Fisher's exact $p=0.1429$ ).

\section{Remaining Distance to Barricade}

A 2 (Age) x 2 (Gender) x 2 (Alert) between-subjects ANOVA was performed on the remaining-distance-to-barricade dependent variable. Drivers that received an FCW alert 
stopped significantly further away from the barricade than drivers that did not receive an FCW alert $(\mathrm{F}(1,14)=26.98, p=0.0001)$. Drivers that received an FCW alert stopped $8.33 \mathrm{~m}$ (s.e. $=1.78 \mathrm{~m}$ ) from the barricade, while drivers that did not receive an FCW alert stopped $28.47 \mathrm{~m}$ (s.e. $=3.52 \mathrm{~m}$ ) from the barricade. Figure 55 presents drivers' mean stopping distance according to their age and gender.

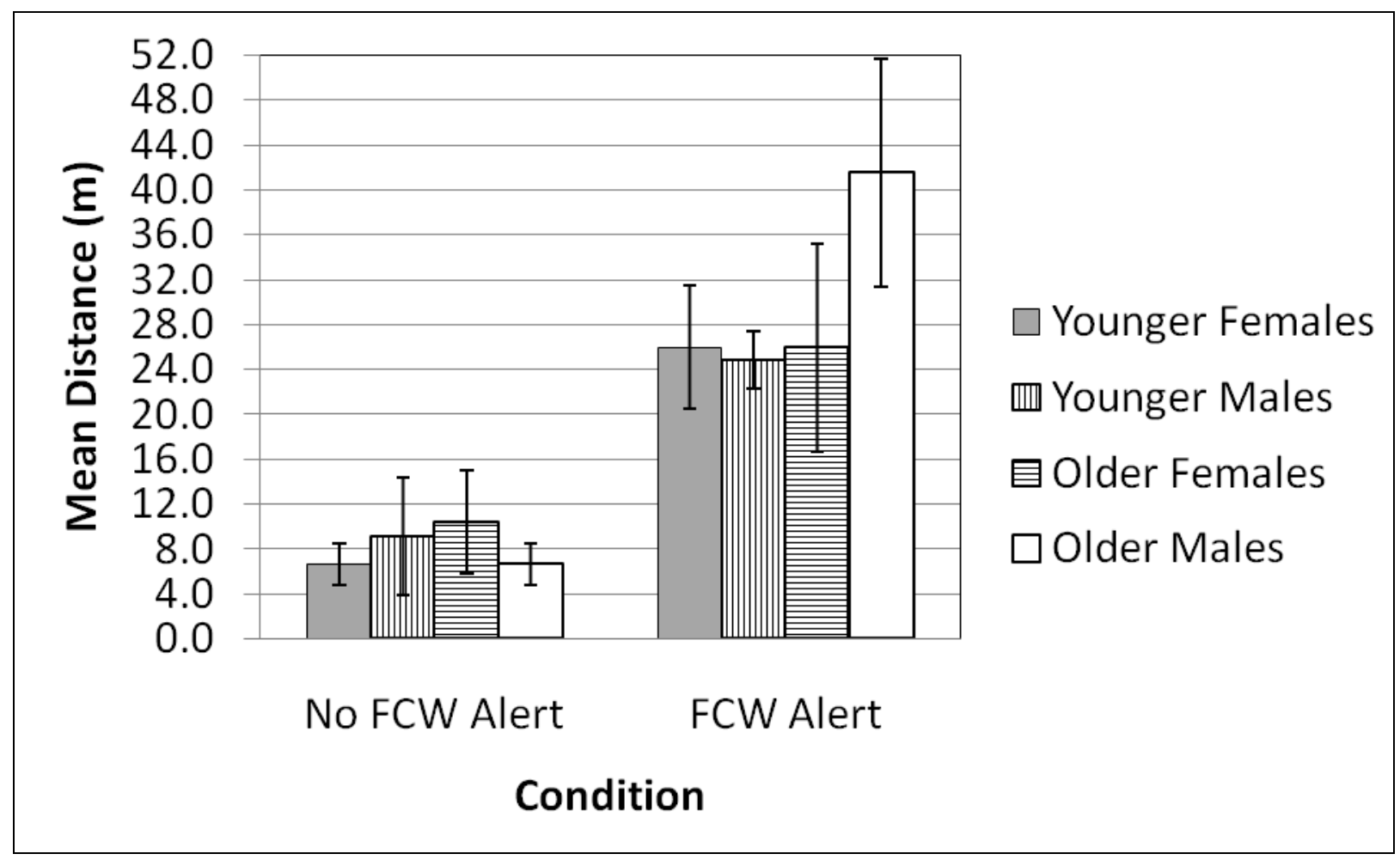

Figure 55. Remaining distance to barricade once vehicle came to a stop.

\section{Peak Deceleration}

A 2 (Age) x 2 (Gender) x 2 (Alert) between-subjects ANOVA was performed on drivers' peak deceleration. No significant differences were found. Drivers who did not receive an FCW alert had a mean peak deceleration of $0.40 \mathrm{~g}$ (s.e. $=0.05 \mathrm{~s}$ ), while drivers that received an alert had a mean peak deceleration of $0.30 \mathrm{~g}(\mathrm{~s} . \mathrm{e}=0.04 \mathrm{~s})$.

\section{Driver Ratings}

This section analyzes drivers' assessments of the unexpected braking event and the FCW alert. The interested reader is referred to Appendix $\mathrm{O}$ for the results of driver's opinions regarding the alert's design.

\section{Question 1: Please rate how distracted you were while the barricade was inflated.}

On average, drivers that did not receive an FCW alert were distracted while the barricade was inflated (mean response $=2.92$, s.e. $=0.43$ ). In contrast, drivers that did receive an FCW alert were neutral when asked how distracted they were while the barricade was 
inflated (mean response $=4.25$, s.e. $=0.59)$. A $2($ Age $) \times 2($ Gender $) \times 2(\mathrm{FCW}$ Alert $)$ between-subjects ANOVA was performed on drivers' distraction ratings. No statistically significant differences were found. Figure 56 presents drivers' ratings of how distracted they were while the barricade inflated broken down by their age and gender.

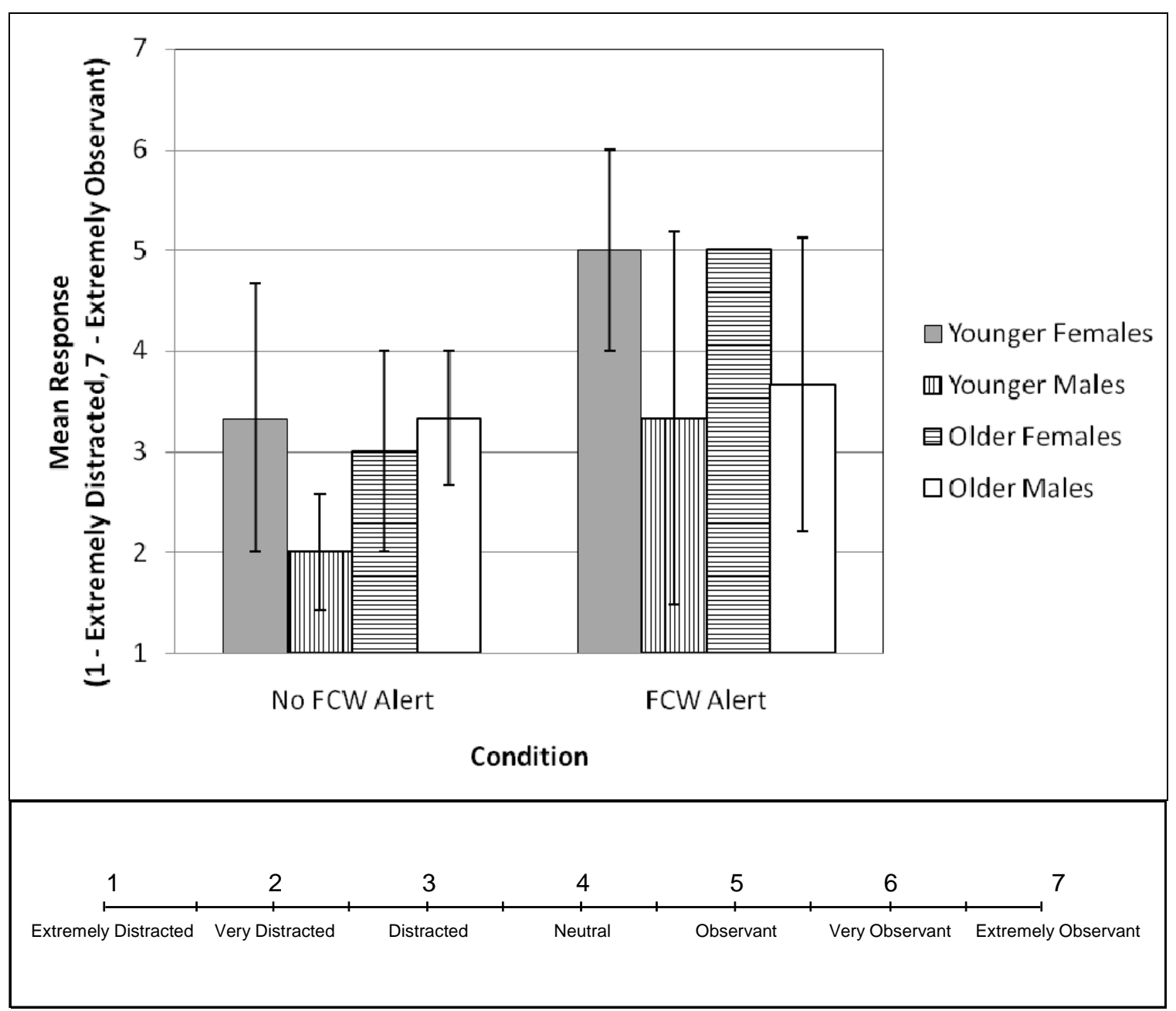

Figure 56. Drivers' ratings of how distracted they were while the barricade inflated.

\section{Question 2: Please rate how surprised you were that you had to stop.}

On average, drivers were surprised that they had to stop to the inflatable barricade after they received the $\mathrm{FCW}$ alert (mean response $=5.08$, s.e. $=0.34$ ). In contrast, drivers reported that they were very surprised when they had to stop to the inflatable barricade without receiving the $\mathrm{FCW}$ alert (mean response $=5.79$, s.e. $=0.26)$. A $2($ Age $) \times 2$ (Gender) x 2 (FCW Alert) between-subjects ANOVA was performed on drivers' surprise ratings. No statistically significant differences were found. Figure 57 presents drivers ratings of how surprised they were that they had to stop broken down by their age and gender. 


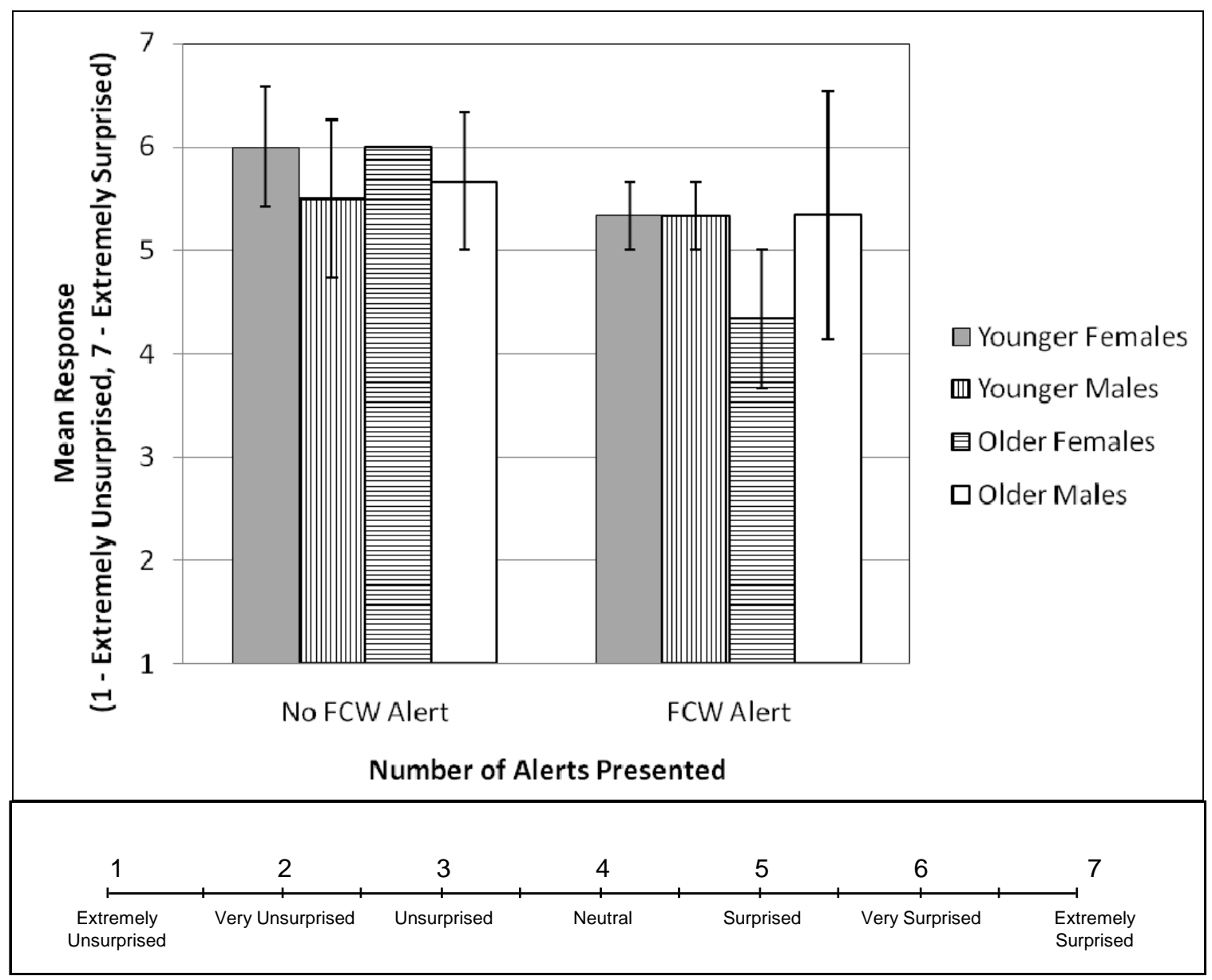

Figure 57. Drivers' ratings of how surprised they were that they had to stop.

\section{Question 3: Please indicate the degree to which the vibration made you look forward using the options listed below}

Seven of the twelve drivers (58 percent) that received an FCW alert as the barricade was presented reported that they looked up immediately in response to the vibration. Three drivers ( 25 percent) reported that they looked up, but only after hesitating due to confusion. Two drivers (17 percent) reported that they did not look up at all after receiving the FCW alert.

\section{DISCUSSION}

This experiment investigated the ability of a haptic driver seat to alert drivers to an actual crash threat. It was found that drivers that received an FCW alert (which consisted of vibrations along the front edge of the driver seat) as a surprise barricade inflated abandoned a distraction task and returned their eyes to the forward roadway sooner than drivers that did not receive an FCW alert. These drivers were also observed to remove their foot from the throttle sooner, as well as press the brake pedal sooner. These drivers brought the vehicle to a stop farther away from the crash threat than drivers that did not 
receive an alert ( $28 \mathrm{~m}$ vs. $8 \mathrm{~m}$ ).

Driver ratings revealed a trend that drivers that did not receive an FCW alert were more distracted than drivers that received an FCW alert at the time the barricade inflated. A trend was also observed that drivers that did not receive an FCW alert were more surprised they had to stop compared to drivers that received an FCW alert. These findings may be because drivers that received an FCW alert looked up sooner and were able to see the tail end of the barricade inflating. These trends help explain the alerting nature of the FCW alert, particularly when drivers are preoccupied with secondary tasks. That is to say, a driver preoccupied with dialing a number on a cell phone, checking email on a PDA, reaching for a beverage, or switching the radio station may be cued to look up at the forward roadway after receiving a haptic alert and detect a forward crash threat sooner than they would without this feedback. Perhaps the FCW alert serves to initiate task switching, the cognitive action behind multitasking, from a secondary task to the primary task of driving (Meyer, et al., 1997).

All but one of the drivers that received an FCW alert pressed the brakes and brought the vehicle to a stop in front of the barricade. Furthermore, six of these 12 drivers were observed to lift their foot off the throttle before they even looked up at the forward roadway to perceive that there was a crash threat. One of these drivers even pressed the brake pedal prior to looking up at the forward roadway. These findings suggest that these drivers may have developed an automatic response to the FCW alert owing to their experience with the haptic alert pattern in Experiment 1.

Driver distraction has been found to significantly elevate crash risk. Specifically, eyeglances away from the forward roadway greater than $2 \mathrm{~s}$ were found to double one's crash risk (Klauer, Dingus, Neale, Sudweeks, \& Ramsey, 2006). Given we are in a era where driver distraction will occur, a haptic driver seat that cues drivers to slow down and return their attention to the forward roadway may prove its worth in situations where drivers fail to perceive developing crash threats owing to involvement in secondary tasks.

By mapping haptic alert patterns and locations to desired manual responses, drivers may be better at determining the appropriate driving action upon receiving an alert, even when they do not see the crash threat. If drivers are developing an automatic response to the alerts, it is foreseeable that these alerts may assist drowsy drivers who fail to perceive a forward crash threat owing to their eyelids covering their eyes. Such a study would be worthwhile pursuing as future research. 


\section{CHAPTER 6. GENERAL DISCUSSION}

This dissertation presents a series of experiments that investigated drivers' ability to comprehend multiple alerts presented through a haptic driver seat. As predicted by established human factors principles, such as the Hick-Hyman law of choice response time (Hick, 1952; Hyman, 1953) and Miller's $7 \pm 2$ numerical limits on immediate memory (Miller, 1956), statistically significant degradations in drivers' performance were observed as the amount of information conveyed by a haptic driver seat was increased. It was found in Experiment 1 that drivers' manual response times to fictitious alerts lengthened, and their manual and verbal response accuracy worsened, as the number of differing alerts increased. It was found in Experiment 2 that drivers' manual response times lengthened, and their manual response accuracy worsened, when the alerts they were instructed to respond to were presented through the same seat location as alerts they were instructed to ignore compared to when both alerts were presented through different locations. The observed performance decrements in these two experiments were quantified. Interestingly, incrementing the alert information was not found to equally affect all drivers. Younger drivers were able to efficiently manage the increased information, while older drivers were less efficient at processing the increased information. These results demonstrate that variation in human performance can be expected when implementing a haptic driver seat. A haptic seat design should therefore be evaluated using participants who range in age prior to being finalized. Despite these performance differences, it was found in Experiment 3 that all drivers benefited from haptic alert feedback to a crash threat.

How do these findings implicate the design of a haptic driver seat? Should the functionality be limited because a segment of the driving population does not perform as well as another (i.e., using a one-alert approach instead of a seven-alert approach)? Or, should an informative seven-alert approach be implemented with the understanding that not all drivers will equally benefit from its design? These are the questions that are appropriate for Human Factors engineering. Human Factors engineering mandates three general design approaches for fitting tasks to humans: 1) design for the population extreme, 2) design for the population mean, and 3) design the system to be adjustable (Kroemer \& Grandjean, 1997). An example of designing for the population extreme would be mounting a water-fountain three feet from the ground so that a person in a wheel-chair can drink from it. Here, taller people can still drink from the fountain by bending down, and since use is infrequent, issues with back pain are traded off for the benefits of accessibility. An example of designing for the population's mean would be setting the height of an office workstation to be appropriate for the average height of the user population. Since use is frequent, the discomfort people experience when working with the workbench that is too low or too high is minimized for the user population by only affecting a small group of extremely tall and short people. Designing systems to be adjustable allows systems to be tailored to the user's needs. This option is the best alternative for preserving users' health and safety. However, adjustability typically comes at an added cost and requires users to know what the appropriate settings are to meet their needs. High-end office chairs adjust to properly fit the user, but the user must know what height, angle, and distance from the desk to position the chair to prevent incursion of musculoskeletal injuries. These settings are not always understood by users 
and so the benefits offered by adjustability are not always realized. In considering these three approaches to Human Factors engineering, design recommendations for a haptic seat alert approach are presented below.

\section{DESIGN RECOMMENDATIONS}

Advancements in automotive technology have made it possible for vehicles to warn drivers of various types of crashes before they occur. In addition, it is imperative that drivers quickly perceive alerts, comprehend them, and make the correct driving responses in order to realize the safety benefits the warning systems stand to offer. A tradeoff exists between providing drivers with information on surrounding crash threats and the degradations in response performance that arise as system complexity increases. Drivers' performance using a one-alert approach, a three-alert approach, and a seven-alert approach was investigated in this dissertation. Based on the findings, a three-alert approach is recommended. Reasons for this recommendation are as follows. First, although drivers' made no mistakes and were fast at responding when using the one-alert approach, the one-alert approach is functionally limited. The three-alert approach surpasses the one-alert approach by alerting drivers to three crash threat directions, while only slightly delaying drivers' response times. Here, the directional information specifies the location of the crash threat, alleviating the burden of determining where the danger lies from the driver. Secondly, although the seven-alert approach offers drivers tremendous improvements in functionality, significant increases in drivers' response times were observed as a result of the added complexity. The three-alert approach, therefore, provides a balance between increased functionality and driver performance. Its simple design also allows it to be effectively used by both older and younger drivers, approximate extremes of the driving population. With it, drivers made the correct driving response 99 percent of the time, took an average of $0.847 \mathrm{~s}$ to initiate their responses, and correctly verbally identified the alerts 99 percent of the time. A three-alert approach might therefore be able to reduce the number of crashes by just indicating crash threat direction to drivers.

The three-alert approach was designed to support drivers' response performance. The following design requirements are submitted to facilitate the replication of these results:

\section{1) High Degree of Stimulus-Response Compatibility}

A reason why performance with the three-alert approach was noteworthy is believed to be because of the stimulus-response compatibility that was designed into the haptic seat. The FCW alert that warned drivers to stop the vehicle was generated by vibrating the front edge of the driver seat. The left LCW alert that warned drivers of danger on the left was generated by vibrating the back-left edge of the driver seat. Similarly, the right LCW alert that warned drivers of danger on the right was generated by vibrating the back-right edge of the driver seat. Here, the location of the alert was mapped to the required driving response, allowing drivers to quickly determine the appropriate response. 


\section{2) Consideration for Drivers' Frame of Reference}

The design of the haptic driver seat took drivers' frame of reference into consideration. A drivers' frame of reference is a point on their body to which they use in conjunction with haptic stimuli to assess which direction the haptic stimuli is intending to orient their attention (Fitch, Kiefer, Kleiner, et al., 2007; Van Erp, 2005). The FCW alert that was intended to orient drivers' attention forward was generated by vibrating the front edge of the driver seat. Had this alert consisted of vibrations elsewhere in the driver seat, drivers' ability to quickly identify its meaning may have been compromised. Likewise, the LCW alerts that warned drivers of lateral threats were generated along the back-side edges of the driver seat. The tactors for these alerts were specifically mounted to press against the side of drivers' hips. This is because the area underneath the drivers' legs is quite close to their frame of reference. Placing these tactors farther away from this central location is believed to have facilitated quick comprehension of their intended meaning.

\section{3) Unique Tactors for Each Alert}

Each alert location was generated by activating unique tactors. The alerts that warned drivers of danger ahead were generated by vibrating the tactors mounted in the front edge of the driver seat. The alerts that warned drivers of danger on the left were generated by vibrating a different tactor mounted in the back-left corner of the seat. Likely, the alerts that warned drivers of danger on the right were generated by vibrating another tactor mounted in the back-right corner of the seat. As a result, there was no confusion between the front, left, and right directions. Other haptic seat studies that did not use unique tactors when presenting alerts reported that some participants were unable to differentiate the alerts (Sayer, et al., 2005). These participants commented that they felt as if "the whole seat buzzed" and that they "couldn't tell which leg was being vibrated." The use of unique tactors in this dissertation is believed to be a reason why such confusion did not arise.

\section{4) Large Distance between Tactors}

A large distance separated the tactors used to generate the three alerts. This distance was believed to facilitate clear perception of which tactors were vibrating. Very few errors occurred in which drivers confused one alert for another. The majority of the errors involved drivers failing to make a response. Had the tactors been located closer together, drivers may have had a harder time distinguishing which tactors were vibrating. Although not explicitly tested, Fitch, Kiefer, Kleiner, et al. (2007) hypothesize that a reason why drivers made haptic seat alert localization errors was because the tactors used were mounted close together in the driver seat.

The above design recommendations should be considered when designing a haptic driver seat to present CAS alerts. An issue to consider when implementing a haptic driver seat is driver training. A haptic driver seat stands to be confusing if it is not explained to drivers. It should be noted that the experimenter explained the haptic driver seat to participants before they began the experiment. Including reference material in the vehicle's instruction manual does not guarantee driver comprehension since few drivers 
read these manuals upon purchasing a vehicle. One way to resolve this issue could be to present a tutorial on the haptic driver seat via a visual display mounted in the center console when the vehicle's ignition is turned on. The tutorial could consist of a voiceguided tour of the haptic seat and the various alerts it generates. Sample alerts could be generated so drivers are not compelled to engage a near-crash to discover what the alerts feel like. After drivers complete the tutorial, they could specify whether they wish the tutorial to be launched the next time the vehicle is turned on. This approach is commonly used in software applications, where the user marks a checkbox that states "do not show me again." Drivers that wish to revisit the tutorial after selecting this option could do so through a graphical user interface menu.

At the time of this dissertation, the American automobile industry is requesting a $\$ 15$ billion emergency loan from the U.S. Government's Troubled Asset Relief Program (TARP) in order to stay viable. Under such economic conditions, investments in active safety systems will undergo excruciating benefit/cost analyses. Although a one-alert approach is the least expensive alternative to implement, the benefits offered by one active safety system may be surpassed by those offered by an integrated alert approach. At the same time, although a seven-alert approach offers the most functionality, it carries the largest price tag, particularly because the involved systems are not yet production ready. The recommendation to adopt a three-alert approach is in alignment with the limited funds available to develop production-ready active safety systems. For example, the three-alert approach could be used to effectively present FCW and LDW alerts, two active safety systems that have undergone testing and are production-ready systems.

\section{SUMMARY}

Active safety promises to bring the next significant reduction in highway fatalities. Automobile equipment manufacturers, government administrations, as well as academic researchers are diligently working to develop systems that prevent fatal automobile collisions from occurring. The research presented in this dissertation provides this design community further insight on a potential crash alert approach; i.e., a haptic driver seat that communicates crash threats to drivers by vibrating various locations in the seat using various vibrotactile patterns. It may also generate discussion on the implications of integrating multiple CASs. This is because this dissertation showed that measurable effects exist when the number of alerts that can be presented to drivers is increased. How active safety systems take these findings into consideration may mean the difference between an effective warning system that saves lives, and a warning system that interferes with driver collision avoidance performance. 


\section{CHAPTER 7. FUTURE RESEARCH}

Researchers concerned with the design and development of a haptic driver seat to communicate CAS alerts may wish to consider the following unexplored areas. The first area deals with the first stage of human information processing, sensation/perception. Research could be performed to determine the effects of varying the distance separating the tactors on drivers' response performance. The optimal distance separating haptic seat tactors, which balances drivers' anthropometry and haptic alert discriminability, should be determined. Using a machine to measure the tactors' frequency and intensity, the optimal intensity to generate alerts could be determined by measuring people's subjective ratings of various frequencies and intensities. Drivers' ratings of the alert's perceived urgency as well as annoyance could be used to assess this specification.

The second area deals with the second stage of human information processing, cognition. The intuitive layout of the haptic driver seat could be confirmed by comparing performance with it to performance with a haptic driver seat that lacks stimulus-response compatibility. The hypothesis that the alerts must consider drivers' frame of reference could also be tested by similarly comparing two haptic seat displays. The implications of generating alerts that share common tactors (such as a "left" alert that consists of vibrating tactors in the back-left and front-left corners of the seat pan and a "front" alert that consists of vibrating tactors in the front-left and front-right corners of the seat pan) could be compared to alerts that utilize unique tactors (e.g., changing the previously mentioned "left" alert to just use the back-left tactor). The effects of increasing the number of alerts could be further explored by investigating more than three levels of display complexity (e.g., adding a ten-alert approach to the experimental design). The Age effects identified in this dissertation could be further explored by examining additional age groups (e.g., ages 40-50 years old). Measuring driver workload as the number of alerts is increased may provide further insight regarding the observed performance decrements. The effectiveness of haptic seat alerts at alerting drowsy, or inebriated drivers is also worth exploring. It would be interesting to see if these drivers exhibited automatic responses to the alerts. Furthermore, it would be worthwhile to compare auditory vs. haptic seat alerts in terms of their ability to grab the attention of drowsy or inebriated drivers.

As recommended by Dingus et al. (1997), the alerts could be tested within a graded alert approach. It is foreseeable that drivers' receiving early stage warnings through a visual display may anticipate a developing crash threat. Recall that Information Theory states that the amount of information conveyed by an alert is dependent on its sequential constraints. The sequential constraints arising from early-stage visual alerts always occurring prior to final-stage haptic alerts would reduce the amount of information conveyed by final-stage haptic alerts. These sequential constraints may lead to shorter response times to the final-stage haptic alerts. For example, a side blind spot notification system stands to improve drivers' awareness of surrounding crash threats by presenting a visual alert when a vehicle is located in the blind spot (Kiefer \& Hankey, 2008). This awareness may improve drivers' expectancy for potential lateral danger when executing a lane change. Their response times to LCW alerts may therefore be shorter. Following the same logic, the haptic seat alerts could be tested with a Camera/Video Imaging 
System (C/VIS), a system designed to improve drivers' spatial awareness of surrounding objects by relaying video images of the vehicle's blind spots to drivers through monitors mounted inside the vehicle cabin (Wierwille, Schaudt, Fitch, \& Hanowski, 2007). Here, monitors that show drivers images of developing crash threats may raise drivers' expectancy for haptic seat alerts, and thus reduce their response times to them. Specifically testing the seven-alert approach with graded alerts or C/VISs is worthwhile, as this may shorten the lengthy response times drivers exhibited when using it.

The third area deals with the final stage of human information processing, response selection. Testing the alerts' ability to support drivers in avoiding actual frontal and lateral crash threats is ultimately desired. Similarly to the logic behind graded alerts, the presence of a developing crash threat may serve as a sequential constraint that an alert is about to generate. An example would be someone driving with a short headway to a lead vehicle who receives an FCW alert while he or she is looking away from the forward roadway. Here, this driver may be predisposed to press the brake pedal when receiving an FCW alert owing to their proximity to a lead vehicle. The existence of a crash context may also foster improved alert comprehension because drivers may be able to associate the generated alert with the crash context facing them. Specifically testing the seven-alert approach with a crash context is worthwhile for these reasons. Testing the alerts under a crash context could be performed through a field operational test, a well-devised experiment using the Virginia Smart Road, or by using a high-fidelity driving simulator. However, testing the alerts through field research would improve external validity compared to testing them in a laboratory because drivers' natural response performance to the alerts when facing a true crash threat could be measured. Research on drivers' ability to comprehend haptic alerts presented through the driver seat, steering wheel, brake pedal, and seat belt should also be explored. Particularly if specific patterns are correlated to each location, the prevention of information loss may be a finding.

Since older drivers' performance degraded more than younger drivers' performance when using the seven-alert approach, future research could explore whether allowing older drivers to step down to a three-alert approach (that communicates threat direction only) from a seven-alert approach (that communicates both threat direction and type) is feasible. It would also be worth exploring whether older drivers show improved performance with a seven-alert approach after they become comfortable with a three-alert approach. Another alternative to address degradations in older driver performance with the seven-alert approach could be to allow drivers to select how early they want the alerts to be generated. Older drivers might select the alerts to be generated earlier on to compensate for their longer response times. A haptic driver seat's settings could be controlled via a visual display commonly found in newer vehicles in the center console. Future research could also explore whether learning the three-alert approach first assists older drivers in utilizing a seven-alert approach.

The findings from this dissertation suggest that a haptic driver seat promises to be an effective means of conveying information to drivers. Investigating the research areas presented in this chapter will help refine the tested communication approach, and advance our understanding of the contexts in which the haptic seat succeeds and fails. 
The development of a haptic seat that effectively conveys information to both younger and older drivers is the ultimate goal. 


\section{CHAPTER 8. REFERENCES}

Barfield, W., \& Dingus, T. A. (1998). Human Factors in Intelligent Transporation Systems. Mahwah, New Jersey: Lawrence Earlbaum Associates.

Battelle (2007). Evaluation of the Volvo Intelligent Vehicle Initiative Field Operational Test. Version 1.3. Task Order 7721. (No. DTFH61-96-C-00077). Washington, D.C.

Boehm-Davis, D. A., Casali, J. G., Kleiner, B. M., Lancaster, J., Saleem, J., \& Wochinger, K. (2007). Pilot performance, strategy, and workload while executing approaches at steep angles and with lower landing minima. Human Factors, 49(5), 759-772.

Boff, K. R., Kaufman, L., \& Thomas, J. (1986). Handbook of Perception and Human Performance (Vol. II). New York: John Wiley and Sons.

Boff, K. R., \& Lincoln, J. E. (Eds.). (1988). Engineering Data Compendium: Human Perception and Performance (Vol. II). Wright-Patterson Air Force Base, Ohio: Harry G. Armstrong Aerospace Medical Research Laboratory.

Bolanowski, S., Gescheider, G., \& Verrillo, R. (1994). Hairy skin: psycholical channels and their physiological substrates. Somatosensory and Motor Research, 11(3), 279-290.

Campbell, J. L., Richard, C. M., Brown, J. L., \& McCallum, M. (2007). Crash Warning System Interfaces: Human Factors Insights and Lessons Learned. Contract No. NH22-02-D-02104 (No. HS 810697 ). Washington, D.C.: National Highway Traffic Safety Administration.

Chapanis, A. (1996). Human Factors in Systems Engineering. Toronto: John Wiley \& Sons, Inc.

Cholewiak, R. W., Brill, J. C., \& Schwab, A. (2004). Vibrotactile localization on the abdomen: Effects of place and space. Perception \& Psychophysics, 66(6), 970987.

Cholewiak, R. W., \& Ciollins, A. (1991). Sensory and physiological bases of touch. In M. A. Heller \& W. Schiff (Eds.), The Psychology of Touch (pp. 22-60). Hillsdale, N. J.: Lawrence Erlbaum Associates.

Cholewiak, R. W., \& Collins, A. A. (2003). Vibrotactile localization on the arm: Effects of place, space, and age. Perception and Psychophysics, 65(7), 1058-1077.

Craig, G., Jennings, S., Cheung, B., Rupert, A., \& Schultz, K. (2004). Flight-test of a tactile situational awareness system in a high-hover task, Baltimore, MD, United States.

DaimlerChrysler Corporation (2007). Chrysler Pacifica 2008 Owner's Manyal (81-0260842). Printed in the U.S.A.

Dingus, T., Klauer, S., Neale, V. L., Petersen, A., Lee, S. E., Sudweeks, J., et al. (2006). The 100-Car Naturalistic Driving Study, Phase II - Results of the 100-Car field experiment. Washington, D.C.: National Highway Safety Administration (NHTSA).

Dingus, T. A., McGehee, D. V., Manakkal, N., Jahns, S. K., Carney, C., \& Hankey, J. M. (1997). Human factors field evaluation of automotive headway maintenance/collision warning devices. Human Factors, 39(2), 216-229. 
Edworthy, J. (1998). What Makes a Good Alarm? IEE Colloquium on Medical Equipment Alarms. The Need, The Standards, The Evidence., 2/1-2/4.

Fitch, G., Kleiner, B., Kiefer, R. J., Lee, S. E., \& Babski-Reeves, K. (2005). Drivers' ability to localize auditory and haptic alarms in terms of speed and accuracy. Unpublished Thesis, Virginia Polytechnic Institute and State University, Blacksburg.

Fitch, G. M., Blanco, M., Morgan, J. F., Rice, J. C., Wharton, A. E., Wierwille, W. W., et al. (In Press). Human Performance Evaluation of Light Vehicle Brake Assist Systems. Contract No. DTNH22-05-D-01019, Task Order \# 8. Washington, D.C.: National Highway Traffic Safety Administration.

Fitch, G. M., Kiefer, R. J., Hankey, J. M., \& Kleiner, B. M. (2007). Toward developing an approach for alerting drivers to the direction of a crash threat. Human Factors, 49(4), 710-720.

Fitch, G. M., Kiefer, R. J., Kleiner, B. M., \& Hankey, J. M. (2007). Identifying the pattern of localization responses with a haptic seat intended to alert drivers to the direction of a crash threat. Paper presented at the Human Factors and Ergonomics Society 51st Annual Meeting, Baltimore, Maryland.

Fitch, G. M., Lee, S. E., Klauer, S., Hankey, J. M., Sudweeks, J., \& Dingus, T. A. (2009). Analysis of Lane-Change Crashes and Near-Crashes (Technical Report No. DTNH22-00-C-07007, Task Order 23). Washington, D.C.: National Highway Traffic Safety Administration.

Fitch, G. M., Rakha, H. A., Arafeh, M., Blanco, M., Gupta, S. K., Zimmermann, R. P., et al. (2008). Safety Benefit Evaluation of a Forward Collision Warning System: Final Report (Contract No. DTNH22-05-D-01019, Task Order 13). Washington, D.C.: National Highway Traffic Safety Administration.

Geldard, F. A. (1960). Some neglected possibilities of communication. Science, 131, $1583-1588$.

Geldard, F. A., \& Sherrick, C. E. (1972). The Cutaneous "Rabbit": A Perceptual Illusion. Science, 178(4057), 178-179.

General Motors Corporation (2005). Automotive Collision Avoidance System Field Operational Test (ACAS FOT) Final Program Report (Technical Report No. DOT HS 809 886). Warren, MI: General Motors Corporation and National Highway Traffic Safety Administration.

Gescheider, G. A., \& O'Malley, M. J. (1983). Vibrotactile forward masking: Evidence for channel independence. Journal of the Acoustical Society of America, 74(2), 474485.

Greene, A. J. (2007). Touch, Haptics, and Proprioception Retrieved 4-06-2007, 2007, from http://www.uwm.edu/ ag/teach_pdf/lecturenotes/perception/12Touch.ppt

Greenspan, J., \& Boloanowski, S. (1996). The psychophysics of tactile perception and its peripheral basis. In L. Kruger, M. Friedman \& E. Carterette (Eds.), Pain and Touch (2nd ed., pp. 394). San Diego: Academic Press.

Hick, W. E. (1952). On the Rate of Gain of Information. Quarterly Journal of Experimental Psychology, 4(11), 11-26. 
Ho, C., Reed, N., \& Spence, C. (2006). Assessing the effectiveness of "intuitive" vibrotactile warning signals in preventing front-to-rear-end collisions in a driving simulator. Accident Analysis and Prevention, 38(5), 988-996.

Ho, C., Tan, H. Z., \& Spence, C. (2005). Using spatial vibrotactile cues to direct visual attention in driving scenes. Transportation Research Part F: Traffic Psychology and Behaviour, 8(6), 397-412.

Hyman, R. (1953). Stimulus Information as a Determinant of Reaction Time. Journal of Experimental Psychology, 45(423-432).

Karwowski, W., \& Marras, W. S. (1999). Noise in Industry: Auditory Effects, Measurement, Regulations, and Management The Occupational Ergonomics Handbook (pp. 1661-1692): CRC Press.

Kiefer, R. J., \& Hankey, J. M. (2008). Lane change behavior with a side blind zone alert system. Accident Analysis \& Prevention, 40(2), 683-690.

Klauer, S. G., Dingus, T. A., Neale, V. L., Sudweeks, J. D., \& Ramsey, D. J. (2006). The Impact of Driver Inattention on Near-Crash/Crash Risk: An Analysis Using the 100-Car Naturalistic Driving Study Data (No. DOT-HS-810-594). Washington, DC: NHTSA.

Kleiner, B. M. (1998). Macroergonomic directions in function allocation. In P. Vink, E. A. P. Koningsveld \& S. Dhondt (Eds.), Human factors in organizational design and management VI (pp. 635-640). Amsterdam: North-Holland.

Kochhar, D. S., \& Tijerina, L. (2006). Comprehension of haptic seat displays for integrated driver warning systems. Paper presented at the Human Factors and Ergonomics Society 50th Annual Meeting, San Fransisco.

Kroemer, K. H. E., \& Grandjean, E. (1997). Fitting the Task the Human: A Textbook of Occupational Ergonomics (Fifth ed.). Philadelphia: Taylor \& Francis.

LeBlanc, D., Sayer, J., Winkler, C., Ervin, R., Bogard, S., Devonshire, J., et al. (2006). Road Departure Crash Warning System Field Operational Test: Methodology and Results (No. UMTRI-2006-9-2). Washington D.C.: The University of Michigan Transportation Research Institute.

Lederman, S. (2008). RE: Mechanoreceptor Sensitivity. In Personal Communication with Greg Fitch (Ed.). Blacksburg, VA.

Lederman, S. J., \& Browse, R. A. (1988). The Physiology and Psychophysics of Touch. In P. Dario (Ed.), Sensors and Sensory Systems for Advanced Robots (Vol. F43, pp. 597). New York: Springer-Verlag.

Lee, J. D., Hoffman, J. D., \& Hayes, E. (2004). Collision warning design to mitigate driver distraction, Vienna, Austria.

Loomis, J. M. (1983). Tactile and visual legibility of seven character sets. Paper presented at the meeting of Psychonomic Society.

Lorna, M. B., Stephen, A. B., \& Helen, C. P. (2005). A First Investigation into the Effectiveness of Tactons. Paper presented at the Proceedings of the First Joint Eurohaptics Conference and Symposium on Haptic Interfaces for Virtual Environment and Teleoperator Systems - Volume 00.

Macefield, V. G. (1998). The Signaling of Touch, Finger Movements and Manipulation Forces by Mechanoreceptors in Human Skin. In J. W. Morley (Ed.), Neural Aspects of Tactile Sensation. Sydney, Australia: North Holland. 
McCrickard, D. S., \& Chewar, C. M. (2005). Designing Attention-Centric Notification Systems: Five HCI Challenges. Technical Report TR-05-13. Blacksburg: Computer Science, Center for HCI, Virginia Tech.

McElheny, M. J. (2005). Multidimensional Warnings: Evaluating Curve Warning Stimuli in an On-Road Environment. Virginia Polytechnic Institute and State University, Blacksburg.

McGrath, B. J., Estrada, A., Braithwaite, M. G., Raj, A. K., \& Rupert, A. (2004). Tactile Situational Awareness System Flight Demonstration Final Report (No. USAARL 2004-10). Pensacola, Florida: Naval Aerospace Medical Research laboratory.

McLaughlin, S. B., Hankey, J. M., \& Dingus, T. A. (2008). A method for evaluating collision avoidance systems using naturalistic driving data. Accident Analysis \& Prevention, 40(1), 8-16.

Merchant, R. E. (2007). Skin, M-I Histology Lecture Notes. Richmond, VA: Medical College of Virginia Campus, Virginia Commonwealth University.

Meridian Kiosks (2007). from http://www.meridiankiosks.com/prod escalade.shtml

Meyer, D. E., Evans, J. E., Lauber, E. J., Rubinstein, J., Gmeindl, L., Junck, L., et al. (1997). Activation of brain mechanisms for executive mental processes in cognitive task switching. Paper presented at the Meeting of the Cognitive Neuroscience Society, Boston, MA.

Miller, G. A. (1956). The magical number seven plus or minus two: Some limits on our capacity for processing information. Phsycological Review, 63, 81-97.

Mortimer, B. J. P., Zets, G. A., \& Cholewiak, R. W. (In Press). Vibrotactile transduction and transducers.

National Highway and Traffic Safety Administration (2007a). from http://www.nhtsa.dot.gov/

National Highway and Traffic Safety Administration (2007b). Traffic Safety Facts: Comparison of Crash Fatalities by Gender and Year From 1996 to 2005 Retrieved January 30, 2008, from http://www-nrd.nhtsa.dot.gov/Pubs/810780.PDF

NHTSA (2005). Automotive Collision Avoidance System Field Operational Test Final Program Report (Final program report No. DOT HS 809 886). Washington, D.C.: General Motors Corporation.

Oakley, I., Kim, Y., Lee, J., \& Ryu, J. (2006). Determining the feasibility of forearm mounted vibrotactile displays, Alexandria, VA, United States.

Perez, C. A., \& Weed, H. R. (1991). Optimization of the relationship between pulse width, pulse frequency and sensation thresholds for vibrotactile information transfer, Orlando, FL, USA.

Sachs, R. M., Miller, J. D., \& Grant, K. W. (1980). Perceived magnitude of multiple electrocutaneous pulses. Percept. Psycophys, 28(3), 255-262.

SAE/Automotive Engineering International (Producer). (2007, March 1st) Active Safety Technology: Paving the Road to Accident-Free Driving Telephone/Webcast.

Sanders, M. S., \& McCormick, E. J. (1993). Human factors in engineering design (6th ed. ed.). New York: McGraw-Hill.

Sayer, T. B., Sayer, J. R., \& Devonshire, J. M. H. (2005, June 27-30). Assessment of a Driver Interface for Lateral Drift and Curve Speed Warning Systems: Mixed Results for Auditory and Haptic Warnings. Paper presented at the 3rd International Driving Symposium on Human Factors in Driver Assessment, 
Training, and Vehicle Design Samoset Resort on the Ocean, Rockport, Maine, USA

Shannon, C. E., \& Weaver, W. (1949). A Mathematical Model of Communication. Urbana, IL: University of Illinois Press.

Tan, A. K., \& Lerner, N. D. (1996). Acoustic Localization of In-Vehicle Crash Avoidance Warnings as a Cue to Hazard Direction (No. DOT HS 808 534). Washington, DC: National Highway Traffic Safety Administration.

Tan, H. Z., Gray, R., Young, J. J., \& Traylor, R. (2003). A Haptic Back Display for Attentional and Directional Cueing. Haptics-e, 3(1).

Tencer, A. F., Kaufman, R., Ryan, K., Grossman, D. C., Henley, M. B., Mann, F., et al. (2002). Femur fractures in relatively low speed frontal crashes: The possible role of muscle forces. Accident Analysis and Prevention, 34(1), 1-11.

Transportation Research Board of the National Academies (2008). Strategic Highway Research Program (SHRP 2) Retrieved November 11th, 2008, from http://www.trb.org/SHRP2/

Vallbo, A. B., \& Johansson, R. S. (1978). The Tactile Sensory Innervation of the Glabrous Skin of the Human Hand. In G. Gordon (Ed.), Active Touch: The Mechanism of Recognition of Objects by Manipulation. Oxford: Pergaomon Press.

Van Erp, J. B. F. (2005). Presenting directions with a vibrotactile torso display. Ergonomics, 48(3), 302-313.

Van Erp, J. B. F., \& Van Veen, H. A. H. C. (2004). Vibrotactile in-vehicle navigation system. Transportation Research Part F: Traffic Psychology and Behaviour, 7(45), 247-256.

Van Erp, J. B. F., Van Veen, H. A. H. C., Jansen, C., \& Dobbins, T. (2005). Waypoint navigation with a vibrotactile waist belt. ACM Transactions on Applied Perception (TAP), 2(2), 106-117

Verrillo, R. T., Bolanowski, S. J., \& Gescheider, G. A. (2002). Effect of aging on the subjective magnitude of vibration. Somatosensory and Motor Research, 19(3), $238-244$.

Verry, R. (1998). Don't take touch for granted: An interview with Susan Lederman. Teching of Psychology, 25(1), 64-67.

Volvo (2005). Volvo Trucks Field Operational Test: Evaluation of Advanced Safety Systems for Heavy Truck Tractors. Washington, D.C.

Weber, E. H. (1978). The Sense of Touch (H. E. Ross \& D. J. Murray, Trans.). New York: Academic Press.

Webster's Ninth New Collegiate Dictionary (1985). Springfield, MA: Merriam-Webster, Inc.

Wickens, C. D., \& Hollands, J. G. (1999). Engineering Psychology and Human Performance (3rd ed.). Upper Saddle River, New Jersey: Prentice Hall.

Wierwille, W. W., Schaudt, W. A., Fitch, G. M., \& Hanowski, R. J. (2007). Development of a performance specification for indirect visibility systems on heavy trucks $S A E$.

www.its.dot.gov (2006). Integrated Vehicle Based Safety Systems - A Major ITS Initiative Retrieved December 17, 2006, from www.its.dot.gov 


\section{APPENDIX A - TELEPHONE SCRIPT AND DRIVER SCREENING QUESTIONNAIRE}

Eligible: Yes No

\section{Note to Researcher:}

Initial contact between participants and researchers may take place over the phone. If this is the case, read the following introductory statement, followed by the questionnaire. Regardless of how contact is made, this questionnaire must be administered verbally before a decision is made regarding eligibility for this study.

\section{Introductory Statement:}

After prospective participant calls or you call them, use the following script as a guideline in the screening interview.

Hello. My name is__and I am a researcher at the Virginia Tech Transportation Institute in Blacksburg, VA. I am recruiting participants for a new driving study being conducted here at the Smart Road. I obtained your contact information from the VTTI internal participant database. If this is something you would like to participate in, would you like me to describe the study?

$<$ If No $>$ Ok, thank you for your time.

$<$ If Yes $>$

The purpose of the study is to evaluate a new in-vehicle technology. If you choose to participate, you will drive a test vehicle on the Smart Road while experiencing vibrations in the driver seat. The vibrations are a part of a new driver information system. You should know that the test vehicle is equipped with cameras and microphones that allow us to collect data of you and your voice. The cameras, however, are very small and are placed out of the way.

This study has four parts to it. First, we would perform a simple vision test. Providing this is passed, we would measure your height, weight, leg length, and leg width. We would then move on to the second part which involves you driving the test vehicle around a closed-course test track. The third part involves filling out some questionnaires. The study takes approximately 2.5 hours at the Transportation Institute to complete. Participants are paid \$20/hr. Please note that for tax recording purposes, the fiscal and accounting services office at Virginia Tech (also known as the Controller's Office) requires that all participants provide their social security number to receive payment for participation in our studies. Does this study sound like something you would be interested in doing, and if so, are you willing to provide your social security number when you come in for the study?

If they indicated that they are not interested: 
Thank you for your time.

If they indicated that they are interested:

That's great. I would like to ask you some questions to see if you are eligible to participate.

\section{Questions}

1. Do you have a valid driver's license? (Criterion to participation: the response must be Yes)

$\square$ Yes $\square$ No

2. How old are you? (Criterion for participation: the response must be between 18 and 25 years old or 65 years and older)

3. Have you had any moving violations in the past 3 years? If so, please explain each case.

$\square$ Yes (Criterion for participation: the driver must not have more than two moving violations in the past 3 years)

Description:

$\square$ No

4. Do you have normal or corrected to normal vision? (Criterion for participation: subject must have normal or corrected to normal vision)

$\square$ Yes $\square$ Yes to corrected normal vision $\quad \square$ No

5. Do you wear glasses or contact lenses when driving?

$\square$ Yes with glasses $\square$ Yes with contact lenses $\square$ Both $\square$ No

If the answer is "Yes with glasses," ask the following question:

If you wear glasses, do you wear transition lenses (lenses tone changes depending on light)?

$\square$ Yes $\square$ No

If the answer is "Yes," ask the following question:

If you wear transition lenses, do you have a pair of glasses with regular lenses and can you still drive?

$\square$ Yes $\square$ No

If "yes" then read the following statement to the subject:

Would you bring the glasses with regular lenses if you decide to participate in the study?

If "no", they are still allowed to participate. 
6. Are you able to drive an automatic transmission vehicle without assistive devices or special equipment? (Criterion for participation: the driver must be able to drive an automatic transmission vehicle without assistive devices)

$\square$ Yes $\square$ No

7. (Females only) Are you currently pregnant? $\quad \square$ Yes $\square$ No

(Criterion for participation: the driver must not be pregnant)

If "yes" then read the following statement to the subject:

Unfortunately you are not eligible for this particular study. The driver must not be pregnant to participate in the study. Thank you for your time. Would you like to be called for future studies?

8. Have you had any eye injuries and/or surgeries (including, but not limited to, LASIK, Radial Keratotomy, and cataract surgery)

Yes: Type of surgery/injury

No

(Criterion for participation: Participants who have had previous eye injuries and/or surgeries cannot participate in this study)

9. Have you had any neck and/or spine injuries or surgeries?

$\square$ Yes: Type of surgery/injury

$\square$ No

(Criterion for participation: Participants who have had previous neck or spine injuries and/or surgeries cannot participate in this study)

10. Have you been involved in any accidents within the past 3 years? If so, please explain. (Criterion for participation: the driver must not have caused an accident in the past 3 years.)

$\square$ Yes

$\square$ No

11. Do you have a history of any of the following? If yes, please explain.

Heart/Cardiovascular Condition $\square$ No $\square$ Yes

Stroke

$\square$ No $\square$ Yes

Brain tumor

$\square$ No $\square$ Yes

Head injury

$\square$ No $\square$ Yes

Epileptic seizures

$\square$ No $\square$ Yes

Respiratory disorders $\quad \square$ No $\square$ Yes 


\begin{tabular}{lrl} 
Motion sickness & $\square$ No & $\square$ Yes \\
Inner ear problems & $\square$ No & \multicolumn{1}{l}{ Yes } \\
Dizziness, vertigo, or other balance problems \\
& $\square$ No & $\square$ Yes \\
Diabetes & $\square$ No $\square$ Yes \\
Migraine, tension headaches & $\square$ No $\square$ Yes \\
Retinal Detachment & $\square$ No $\square$ Yes \\
Advanced Osteoporosis & $\square$ No $\square$ Yes
\end{tabular}

(Criterion for participation: subject cannot have lingering effects of heart/cardiovascular condition, brain damage from stroke, tumor, head injury, recent concussion, or infection. Cannot have had epileptic seizures within 12 months, current respiratory disorders, motion sickness, inner ear problems, dizziness, vertigo, balance problems, diabetes for which insulin is required, chronic migraine or tension headaches. Also cannot have retinal detachment or advanced osteoporosis)

12. Are you currently taking any medications on a regular basis? If yes, please list them.

$$
\begin{aligned}
& \square \text { Yes } \\
& \square \text { No }
\end{aligned}
$$

(Criterion for participation: subject cannot currently be taking any substances that may interfere with driving ability, cause drowsiness, or impair motor abilities.)

13. Are you eligible for employment in the United States? (Driver must be eligible for employment in the US)

$\square$ Yes $\square$ No

\section{Note to Researcher:}

If a response to any of the first 16 questions does not meet its criterion, read the following:

Unfortunately you are not eligible for this particular study. Thank you for your time. Would you like to be called for future studies?

Criteria For Participation:

1. Must hold a valid drivers license.

2. Must be between 18-25 or 65+ years old.

3. Must not have more than two moving violations in the past three years.

4. Must have normal (or corrected to normal) vision.

5. Must be able to drive an automatic transmission vehicle without assistive devices. 
6. Must not be pregnant.

7. Must not have caused an injurious accident in the past three years.

8. Cannot have lingering effects of heart condition, brain damage from stroke, tumor, head injury, neck injury, spine injury, recent concussion, or infection. Cannot have had epileptic seizures within 12 months, current respiratory disorders, motion sickness, inner ear problems, dizziness, vertigo, balance problems, diabetes for which insulin is required, chronic migraine or tension headaches. Cannot have had eye surgery (including radial keratotomy). Cannot have retinal detachment or had previous treatment for retinal detachment. Cannot have advanced osteoporosis.

9. Cannot currently be taking any substances that may interfere with driving ability, cause drowsiness, or impair motor abilities.

10. Must be eligible for employment in the U.S. and willing to provide their social security number when they come in.

11. Cannot have participated in a previous braking study at VTTI. (Don't ask them if they were in a previous surprise study. Confirm by checking the participant database.) 
Once the researcher determines that the participant is eligible for the study, or wishes to participate in another VTTI study:

I would like to write down your name, phone number or phone numbers where you can be reached as well as the hours/days when it's best to reach you.

Name

Male/Female

Phone Numbers:

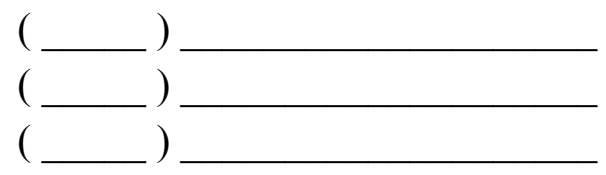

$$
\begin{aligned}
& \square \text { Home } \quad \square \text { Work } \quad \square \text { Cell } \\
& \square \text { Home } \quad \square \text { Work } \quad \square \text { Cell } \\
& \square \text { Home } \quad \square \text { Work } \square \text { Cell }
\end{aligned}
$$

Best time to call: $\square$ Any $\square$ Mornings $\square$ Afternoons $\square$ Nighttime $\square$ Weekends $\square$ Other:

Eligible for Vibrating Seat study? Yes No

Scheduled for the Vibrating Seat study: Date Time

Place in contact list for other VTTI studies? Yes

No

If they are not eligible due to medical conditions, encourage participants to participate in other VTTI studies that does not screen for that condition.

I would like to set up a time when you can come to VTTI and participate in this study. Would it be possible for you to come in on (day of week) at hrs (time)?

If the response is yes, go ahead and schedule the participant and update the 'scheduled participant list.xls' excel sheet with his/her information.

If the response is no, ask the following to the participant:

What day and time would be convenient for you?

If requested day and time is available then schedule the participant and update the 'scheduled participant list.xls' excel sheet with his/her information. If requested day and time is not available then suggest closer day and time slots and see if that will work for the participant.

Once the researcher has scheduled the participant and updated his/her information in the "scheduled participant list.xls" excel sheet then repeat the schedule day and time back to the participant. 
Great! I have you scheduled for (day) at hrs.

I will be calling you a day before to remind you of your schedule. If you need to cancel or reschedule, please call me at 540-

Here are the directions to the Institute. I can also email them to you if you wish.

From I-81:

1. Take exit $118 B$ onto US-460 W towards Christiansburg.

2. Continue on US-460 W for approximately 10 miles.

3. Take exit 5AB toward US-460-BR W/US-460-BR E. The sign for this exit will read "Smart Road Center/Control Center.

4. Stay to your right on the exit ramp until you come to a stop sign at Industrial Park Drive.

5. Turn right onto Industrial Park Dr.

6. Take an immediate right onto Transportation Research Dr.

7. Turn left onto Transportation Research Plaza.

8. Drive up to the building

When you come to institute you may park in any open space available and walk to the new building, which is only one level tall. On the front door you will see a flyer reading "vibrating seat study here". You may enter the first door and wait for the experimenter to open the second door for you. The experimenter will be there to greet you a few minutes before your scheduled time. If you do not see anybody, please wait and an experimenter will be with your shortly.

We ask that all subjects refrain from drinking alcohol and taking any substances that will impair their ability to drive prior to participating in our study.

Please bring your driving glasses for the study.

Do you have any questions that I can answer for you? (Answer the questions if any).

Great then I'll see you on (day) at hrs for the study. Thanks. Have a good day. 


\section{APPENDIX B - VIRGINIA SMART ROAD}

The Virginia Smart Road is a controlled test bed designed for ITS, human factors, and safety research. The research support infrastructure of the facility makes it an ideal location for safety and human factors evaluation. The road is built to Virginia Department of Transportation and Federal Highway Administration standards. The Smart Road has a large number of features and capabilities and is highly adaptable.

\section{WEATHER-MAKING CAPABILITY}

The facility is capable of producing snow, fog-like mist, or rain over a 0.5 -mile stretch of roadway under suitable temperature and wind conditions. At maximum output, the system can produce $10 \mathrm{~cm}$ (4 inches) of snow per hour for 1 hour (Figure 58). A 1900 kilolitre (500,000-gallon) water tank feeds 76 weather towers and allows for multiple research events. The all-weather testing towers' output is automatically controlled from VTTI's control room and can produce snow, rain, fog, or mist at varying intensities. Recently, VTTI has configured portable all-weather testing towers to further enhance the facility's flexibility for research customization. In addition, water can be sprayed by the towers onto freezing pavement to create icy conditions.

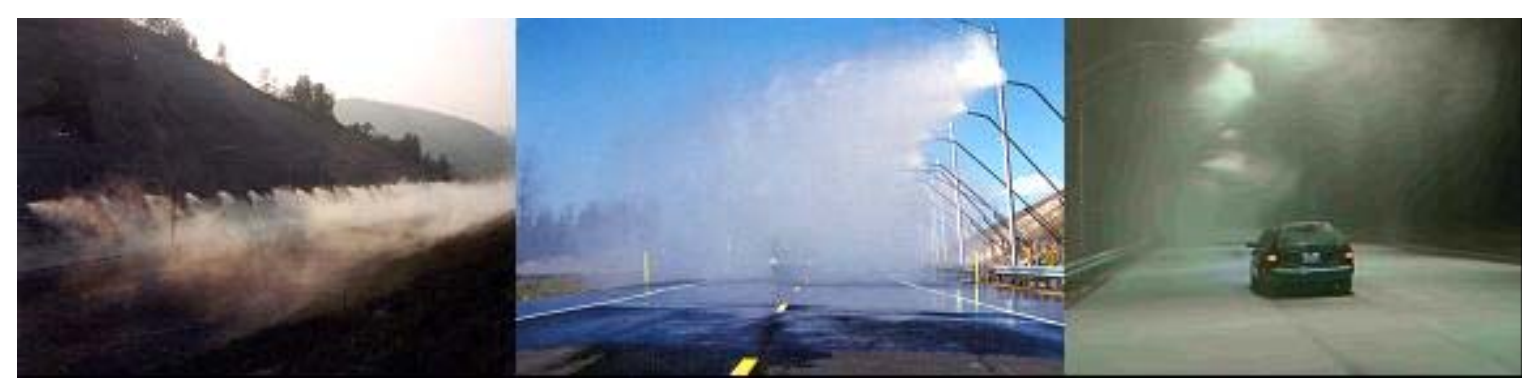

Figure 58. Fog, rain, and snow equipment installed on the Smart Road.

\section{VARIABLE LIGHTING TEST BED}

A highway lighting test bed is also incorporated within the Smart Road. The system consists of 36 overhead light poles that span a 1.1-mile section of the road. The pole spacing pattern is: 40-20-20-40-40-20-20-40-40-20-20 m. This spacing, combined with the wiring of the poles on three separate circuits, allows for evaluation of lighting systems with spacings of 40,60,80 or $120 \mathrm{~m}$. The poles incorporate a modified design to allow for easy height adjustment of the bracket arm. In addition to evaluating spacing and bracket height, various luminaires are also available, including metal halide and highpressure sodium. Additional poles are mounted on portable bases that allow the simulation of other environments as needed (e.g., crosswalks).

The combination of weather-making capabilities and the variable lighting test bed can simulate over 90 percent of the highway lighting in the United States and allows for a variety of different visibility conditions to be created for testing purposes (Figure 59). 


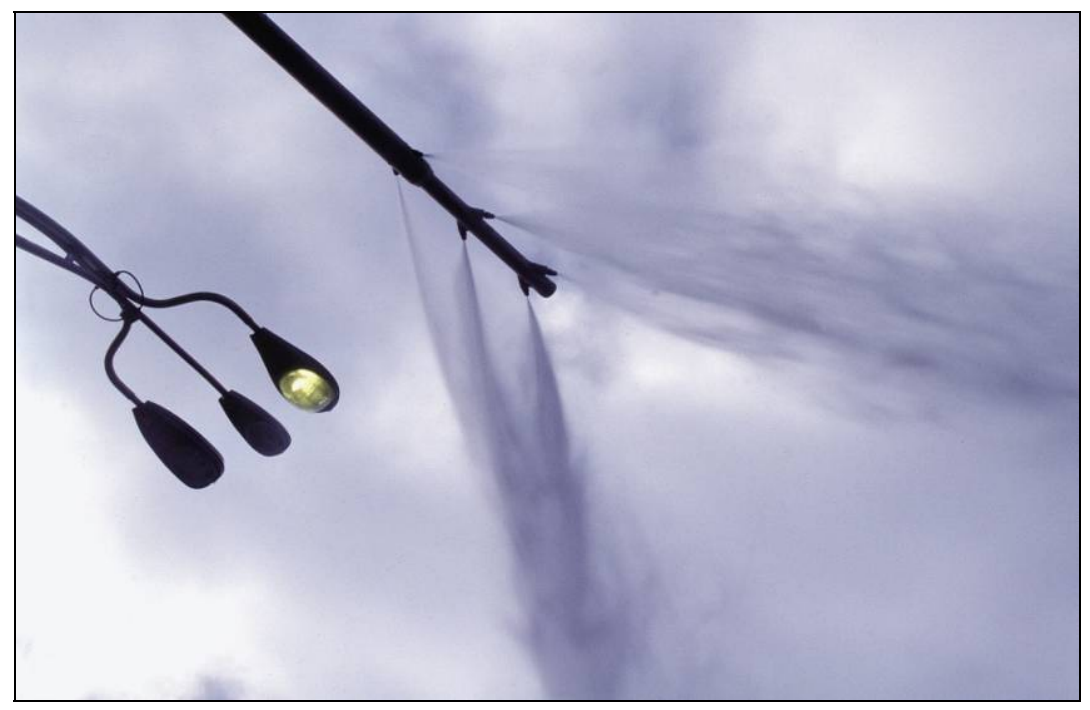

Figure 59. All-weather testing equipment with experimental lighting test bed installed on the Smart Road.

\section{PAVEMENT MARKINGS}

The road includes an additional visibility testing section. This section has been used with a variety of pavement markings for visibility testing. Periodically, as specific studies require it, the markers are reconfigured. Markers on the road may also be reconfigured or repainted as needed. Past research on pavement markings has included UV-reflective markings, prototype reflective mixtures for markings, three-dimensional markings, and installation quality effects on marking visibility.

\section{ON-SITE DATA ACQUISITION AND ROAD WEATHER INFORMATION SYSTEMS}

The roadway has an underground conduit network with an access port (bunker) every $60 \mathrm{~m}$. This network houses a fiber-optic data network and interfaces with several on-site DASs and road feature controls. The facility has a complement of road weather information system sensors connected to the data network. In addition, the road is outfitted in its entirety with a wireless network that ties into the research building's data network. This network may be used for data transfer between the vehicle, the research building, and infrastructure within the road (Figure 60). 


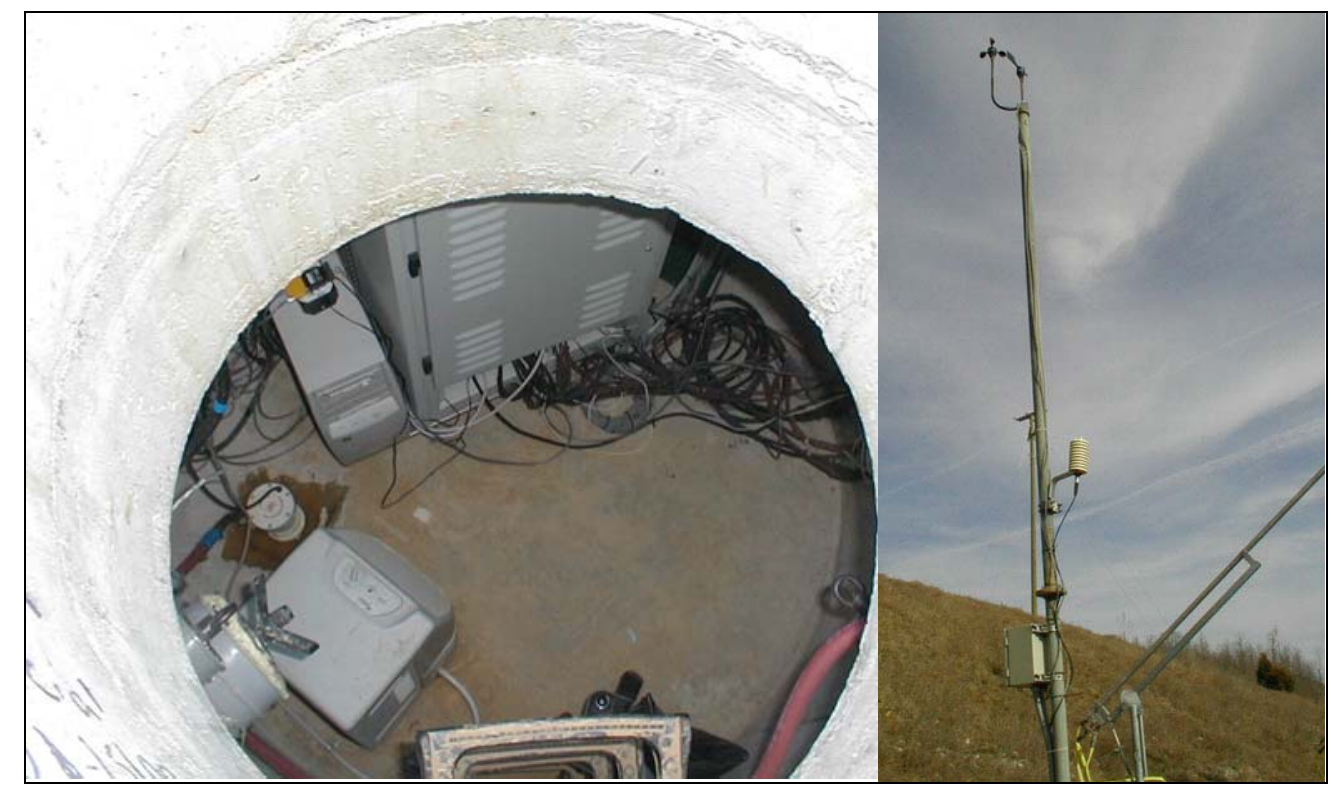

Figure 60. Bunker with DAS and weather station installed on the Smart Road.

\section{DIFFERENTIAL GPS SYSTEM}

Differential GPS corrections are broadcast from the research building to the road. Experimental vehicles are equipped with portable GPS units that, combined with the differential GPS corrections, allow for extremely accurate (on the order of $\pm 1.5 \mathrm{~cm}$ ) onroad vehicle positioning. VTTI has a number of portable differential GPS units available and thus is able to quickly outfit any vehicle for GPS positioning to enhance studies.

\section{ROAD ACCESS AND SURVEILLANCE}

The Smart Road is closed to live traffic, which allows for a variety of different scenarios to be created for testing purposes in relative safety. During past research, for example, experimenters have placed objects of differing size, contrast, and reflectivity on the road to determine the driver's ability to detect them under a wide range of conditions. The lack of live traffic, however, does not prevent the simulation of crash scenarios. Some research projects have used vehicle mockups and appropriately timed distractions to generate surprise conditions. Other projects have employed trained experimenters that act as a pretend maintenance crew. This last method creates the illusion of possible traffic conflicts for participants without any decrease in their safety.

In order to keep the road free of live traffic, vehicle access to the road is restricted with a gate that is controlled from the research building (Figure 61). In addition, the road is outfitted with a video surveillance system that is monitored from the research building 24 hours a day, 7 days per week. This video surveillance system also allows for visual confirmation of vehicle and personnel locations on the road during ongoing studies. 


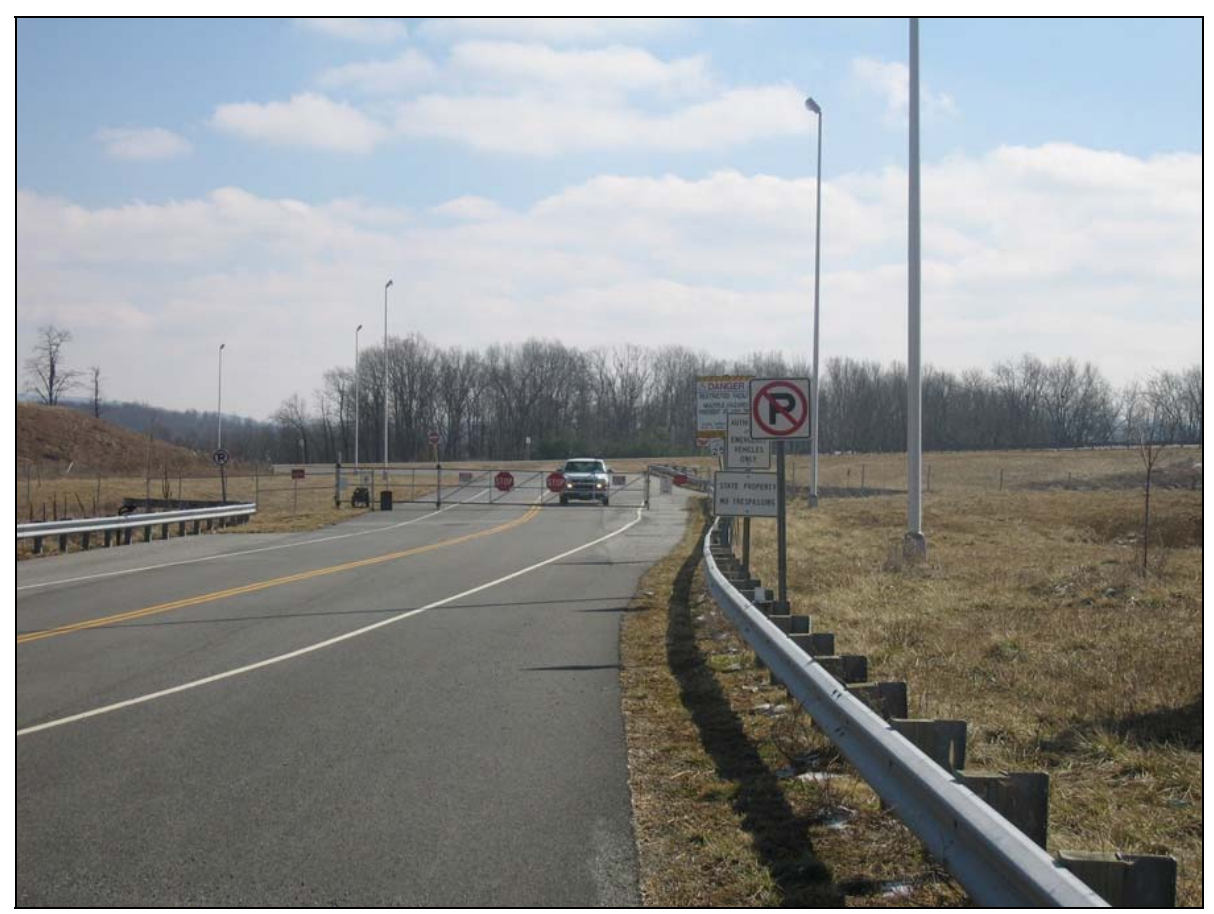

Figure 61. Gate that restricts access to the Smart Road.

\section{RESEARCH BUILDING AT THE SMART ROAD}

The main offices and laboratories of the Virginia Tech Transportation Institute are located within two research buildings located adjacent to the Smart Road. The first research building has three floors encompassing over 2700 square meters $(29,000$ square feet) of office, garage, and specialized laboratory space. In addition to the control room and the garage, discussed in the following paragraphs, this research building contains office space for research and administrative staff, conference facilities, multiple laboratories, and work areas for students. The second building is a recently constructed 2100 square meter (23,000-square-foot) building that is accompanied by a warehouse with four additional garage bays.

\section{CONTROL ROOM}

The control room serves as the core control and monitoring center for the Smart Road (Figure 62). Vehicular access to the Smart Road is managed at all times by a dispatcher who has visual contact with all sections of the road through direct line-of-sight and through a set of surveillance cameras. This dispatcher also activates, as required, controls for lighting and weather. All research efforts using the Smart Road are coordinated and monitored through the control room with a primary focus on safety and security. To aid the dispatcher in monitoring all Smart Road operations, the control room houses a $3 \mathrm{~m}$ $(10 \mathrm{ft})$ by $2.3 \mathrm{~m}(7.5 \mathrm{ft})$ video wall, a projection screen, and up to 12 monitors. 


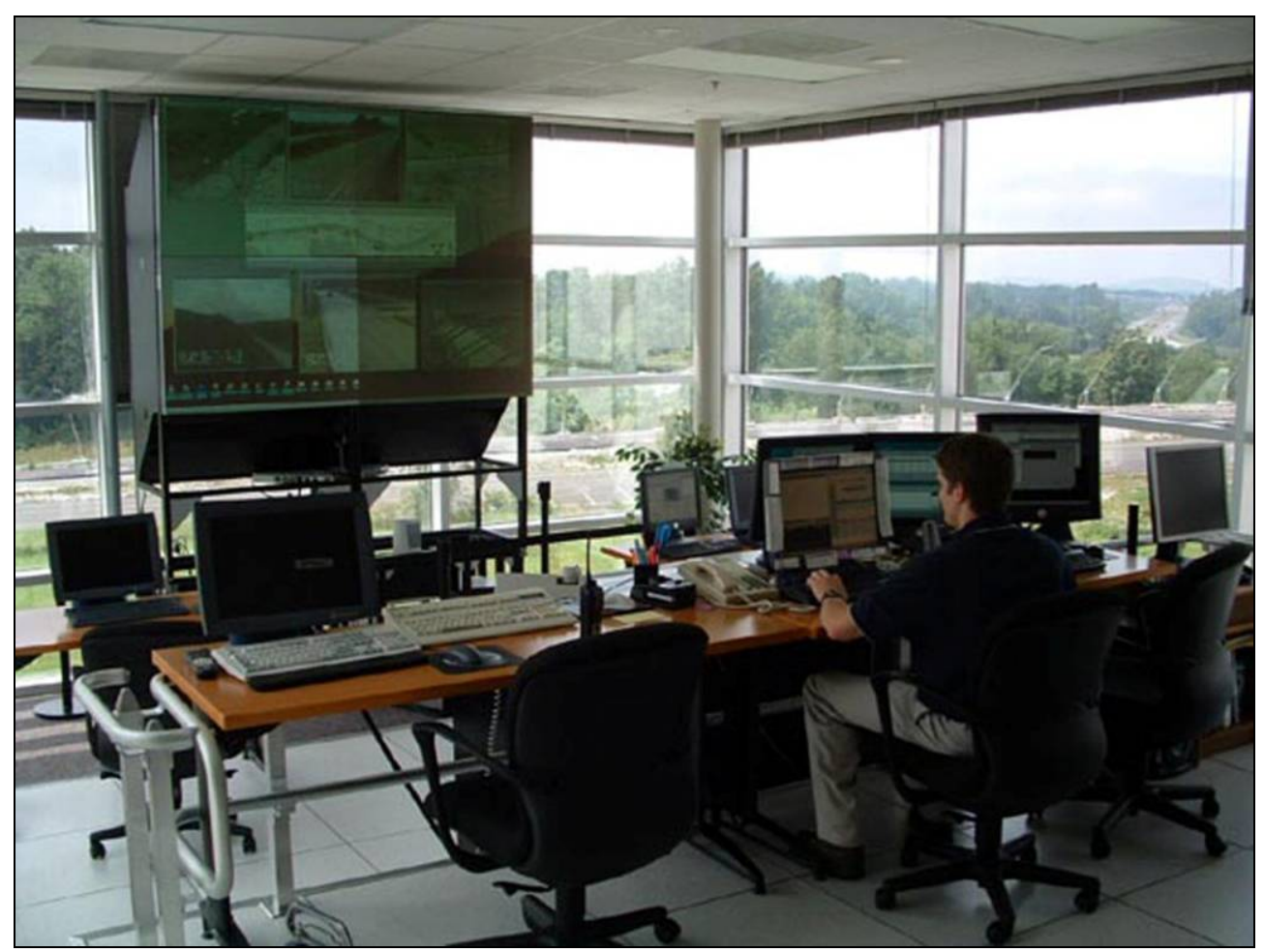

Figure 62. Smart Road control room and dispatcher monitoring research.

\section{GARAGES}

Two garage bays are present in the main building along with machine and electronics shops. The warehouse contains four additional garage bays. All bays have oversized outside doors tall enough to accommodate a semi-tractor. In addition, all of the garages can be isolated in case they need to be used for confidential research, as contractordedicated facilities, or as separate tool and work rooms. These six garages also lack windows to ensure privacy when it is needed by the sponsor.

\section{LABORATORIES}

The building has space allocated for multiple laboratories, including driver interface development, eyeglance data reduction, lighting research, accident analysis, accident database analysis, pavement research, and traffic simulation. Rooms are also available to host focus groups.

\section{VEHICLE FLEET}

VTTI has a variety of vehicles that are used for vehicle research (Figure 63). These vehicles are outfitted with basic instrumentation packages that can be quickly tailored to the specifications of a particular project. The vehicles are capable of recording a variety of data in real time from a suite of sensors and cameras that are inconspicuously mounted. The vehicles include: 
- 2002 Ford Econoline - Mobile Traffic Laboratory.

- 2002 Cadillac Escalade.

- 2002 Cadillac Seville.

- 2002 Chevrolet Cavalier.

- 2001 Saab 9-5.

- 2000 Chevrolet Impala.

- 2000 Ford Explorer, including attachments to test alternate headlamp configurations.

- 1999 Ford Contour.

- 1999 Ford Crown Victoria.

- 1999 Ford Explorer, including attachments to test alternate headlamp configurations.

- 1997 Ford Taurus.

- 1995 Oldsmobile Aurora.

- 1997 Volvo, VN series, class 8 tractor, along with a $14.63-\mathrm{m}$ (48-ft) trailer.

- 1994 Peterbilt model 379 with sleeper.

All of these vehicles have been used in a number of safety and human factors experiments. Experimental areas that have been studied with them include in-vehicle displays, driver distraction, collision warning and avoidance, fatigue assessment, navigation systems, and use of in-vehicle devices. In addition to these vehicles, VTTI owns a small number of experimental support vehicles, such as pickup trucks and passenger vans.

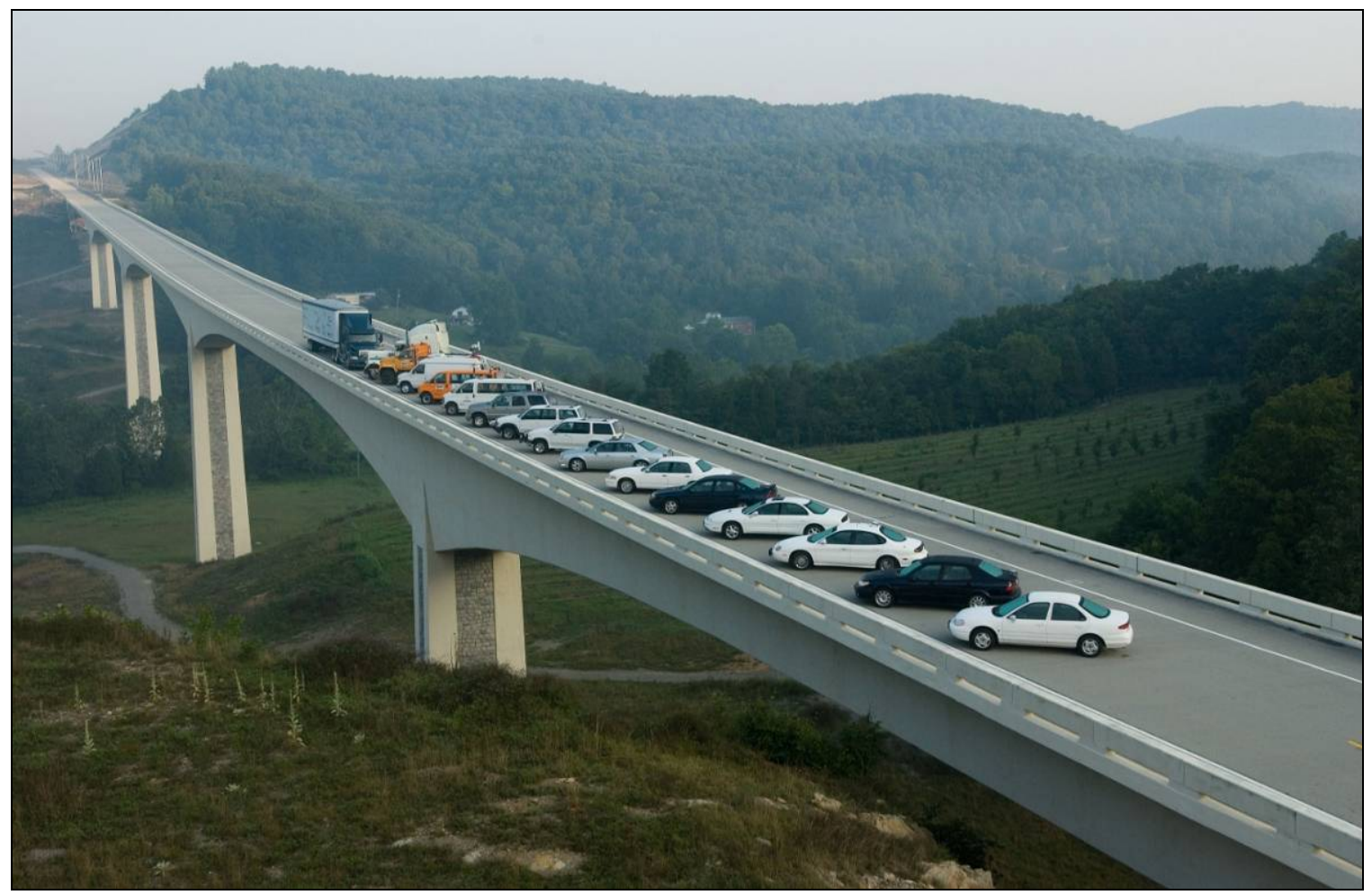

Figure 63. VTTI's vehicle fleet on the Smart Road bridge. 


\section{VEHICLE INSTRUMENTATION}

Over the last 15 years and most recently as part of its efforts during the 100-Car Naturalistic Driving study, VTTI has designed and developed a self-contained vehicle DAS. The system contains a combination of commercial off-the-shelf and in-house components.

The core of the DAS is a Pentium-based PC104 computer. The computer runs custom data acquisition software and communicates with a distributed data acquisition network. Each node on the network contains an independently programmable micro-controller capable of controlling or measuring a moderate number of signals. This system configuration maximizes flexibility while minimizing the physical size of the system. The system is capable of managing up to 120 nodes, but only 10 are used in the current configuration. 


\section{APPENDIX C - INFORMED CONSENT FORM}

\section{VIRGINIA POLYTECHNIC INSTITUTE AND STATE UNIVERSITY Informed Consent for Participants of Investigative Projects}

Title of Project: SAFETEA LU SR - Lighting and Warning System (LWS)

Investigators: Greg Fitch, Jon Hankey, and Brian Kleiner

\section{The Purpose of this Research/Project}

This study will look at whether information can be presented to drivers through vibrations in the car seat. This type of display is called a haptic display, and it allows drivers to keep their eyes on the road. This study may help designers develop haptic displays that let drivers know the direction of a crash, or which way to turn to reach their destination. This study will have 24 participants.

\section{Procedures}

During the course of this experiment you will be asked to perform the following tasks:

1) Read this Informed Consent Form and sign it if you agree to participate.

2) Show your valid driver's license.

3) Complete a vision test and measure your height, weight, leg length, and leg width.

4) Drive an instrumented vehicle at $20 \mathrm{mph}$ on the Smart Road (closed-course test track).

5) Feel haptic seat alerts while driving and make appropriate driving responses (braking or steering)

6) Tell the experimenter when you feel the alerts. Say which alert you felt.

7) Read a display while driving.

8) An experimenter will sit in the back right seat of the vehicle. A video and audio recording will be made to allow for later analysis of your eye movements and verbal responses.

9) Complete questionnaires.

It is important for you to understand that we are not evaluating you or your performance in any way. You are helping us evaluate a haptic driver seat display. Any tasks you perform, or opinions you have will only help us do a better job of designing this system. Therefore, we ask that you perform to the best of your abilities. The information and feedback that you provide is very important to this project. The experiment will last about 2.5 hours. 


\section{Risks}

There are risks or discomforts to which you may be exposed in volunteering for this research. They include the following:

1) The risk of an accident normally associated with driving an unfamiliar vehicle at 20 mph.

2) Possible discomfort from feeling a vibrating driver seat.

3) Possible fatigue due to the length of the experiment.

4) The additional risk of an accident that might occur while viewing any displays (e.g., radio, speedometer, GPS navigation display).

5) While you are driving the vehicle, cameras will videotape your face and eye movements. Due to this fact, we ask that you not wear sunglasses. If this, at any time, impairs your ability to drive the vehicle safely, you are instructed to notify the experimenter.

6) Some studies at VTTI involve an unanticipated event. You may or may not encounter such an event during this study. Please be aware that equipment failure, changes in the test track, stray or wild animals entering the road, and weather changes may require you to respond accordingly. The appropriate response may or may not involve rapid deceleration.

The following precautions will be taken to ensure minimal risk to you:

1) You may take breaks or decide not to participate at any time.

2) An experimenter will be present in the back right seat of the vehicle. However, as long as you drive the research vehicle, it remains your responsibility to drive in a safe and legal manner.

3) The vehicle is equipped with a driver's side and passenger's side airbag supplemental restraint system, fire extinguisher and first-aid kit. The experimenter has a cell phone.

4) All data collection equipment is mounted such that, to the greatest extent possible, it does not pose a hazard to you in any foreseeable case.

5) All testing will be performed on dry test track conditions during daylight hours.

6) You are required to wear the seat and lap belt restraint system while in the car.

7) In the event of a medical emergency, or at your request, VTTI staff will arrange medical transportation to a nearby hospital emergency room. The cost of this transportation would be covered by whichever insurance policy covers the incident causing the medical emergency (see examples in the next section).

8) If you are pregnant, you are not allowed to participate.

9) You do not have any medical condition that would put you at a greater risk, including but not restricted to: neck/spine injury andlor surgery, epilepsy, balance disorders, lingering effects of head injuries and stroke, eye injuries and lor surgeries, retinal detachment, and advanced osteoporosis.

In the event of an accident or injury in an automobile owned by Virginia Tech, the automobile liability coverage for property damage and personal injury is provided. The total policy amount per occurrence is $\$ 2,000,000$. This coverage (unless the other party was at fault, which would mean all expense would go to the insurer of the other party's 
vehicle) would apply in case of an accident for all volunteers and would cover medical expenses up to the policy limit. For example, if you were injured in an automobile owned or leased by Virginia Tech, the cost of transportation to the hospital emergency room would be covered by this policy.

Participants in a study are considered volunteers, regardless of whether they receive payment for their participation; under Commonwealth of Virginia law, worker's compensation does not apply to volunteers; therefore, if not in the automobile, the participants are responsible for their own medical insurance for bodily injury. Appropriate health insurance is strongly recommended to cover these types of expenses. For example, if you were injured outside of the automobile owned or leased by Virginia Tech, the cost of transportation to the hospital emergency room would be covered by your insurance.

\section{Benefits of this Project}

While there are no direct benefits to you from this research, you may find the experiment interesting. No promise or guarantee of benefits is made to encourage you to participate. Participation in this study will contribute to the improvement of haptic displays.

\section{Extent of Anonymity and Confidentiality}

The data gathered in this experiment will be treated with confidentiality. Shortly after participation, your name will be separated from your data. A coding scheme will be employed to identify the data by participant number only (e.g., Participant No. 1). You will be allowed to see your data and withdraw the data from the study if you so desire, but you must inform the experimenters immediately of this decision so that the data may be promptly removed. At no time will the researchers release data identifiable to an individual to anyone other than VTTI staff working on the project without your written consent. VTTI will not release the digital video of your image or audio of your voice without your permission.

It is possible that the Institutional Review Board (IRB) may view this study's collected data for auditing purposes. The IRB is responsible for the oversight of the protection of human subjects involved in research.

\section{Compensation}

You will be paid $\$ 20.00$ per hour for participating. You will be paid at the end of this study in cash. If you choose to withdraw before completing all scheduled experimental tasks, you will be compensated for the portion of time of the study for which you participated. If these payments are in excess of $\$ 600$ dollars in any one calendar year, then by law, Virginia Tech is required to file Form 1099 with the IRS. For any amount less than $\$ 600$, it is up to you as the participant to report any additional income as Virginia Tech will not file Form 1099 with the IRS. 


\section{Freedom to Withdraw}

As a participant in this research, you are free to withdraw at any time without penalty. If you choose to withdraw, you will be compensated for the portion of time of the study for which you participated. Furthermore, you are free not to answer any question or respond to experimental situations without penalty. If you choose to withdraw while you are driving on the test route, please inform the experimenter of this decision and he/she will provide you with transportation back to the building.

\section{Approval of Research}

Before data can be collected, the research must be approved, as required, by the Institutional Review Board for Research Involving Human Subjects at Virginia Polytechnic Institute and State University and by the Virginia Tech Transportation Institute. You should know that this approval has been obtained. This Form is valid for the period listed at the bottom of the page.

\section{Subject's Responsibilities}

If you voluntarily agree to participate in this study, you will have the following responsibilities:

1. To follow the experimental procedures as well as you can.

2. To inform the experimenter if you have difficulties of any type.

3. To wear your seat and lap belt.

4. To abstain from any substances that will impair your ability to drive.

5. To obey traffic regulations and maintain safe operation of the vehicle at all times.

6. To treat the driving task as the primary task and perform other tasks only when it is safe to do so.

\section{Consent to Use Video/Audio Data for Research Reporting Purposes}

Digital video cameras and audio recordings will document your driving behavior. These digital video and audio files will be used to clarify the experimental methods used and to report findings at technical conferences and for other presentations. We are asking you for your permission to show and release portions of videotape displaying your image and audio containing your voice when useful for research or research reporting purposes such as at conferences and technical presentations. The purpose of the following part is to obtain your permission to do so. If you agree, please make a check mark in the box below that best represents your opinion. If you do not agree, your data will not be shown and you will still be compensated for your time. 
Check one of the following:

VTTI has my permission to use the digital video and audio including my image and voice for research or research reporting purposes (such as presentations and at conferences). I understand that VTTI will only use the video and audio data for these purposes.

VTTI does not have my permission to show the digital video and audio including my image for research or research reporting purposes. I understand that VTTI will maintain possession of the video and audio data for research purposes.

\section{Participant's Permission}

I have read and understood the Informed Consent and conditions of this project. I have had all my questions answered. I hereby acknowledge the above and give my voluntary consent for participation in this project. If I participate, I may withdraw at any time without penalty. I agree to abide by the rules of this project.

\begin{tabular}{llc}
\hline Participant's Name (Print) & Signature & Date
\end{tabular}

\begin{tabular}{lll}
\hline Experimenter's Name (Print) Signature Date & Sa
\end{tabular}

Should I have any questions about this research or its conduct, I may contact:

Greg Fitch

Jon Hankey

Brian Kleiner

David Moore (Institutional Review Board Chair)
231-1043

231-1512

231-4926

231-4991 


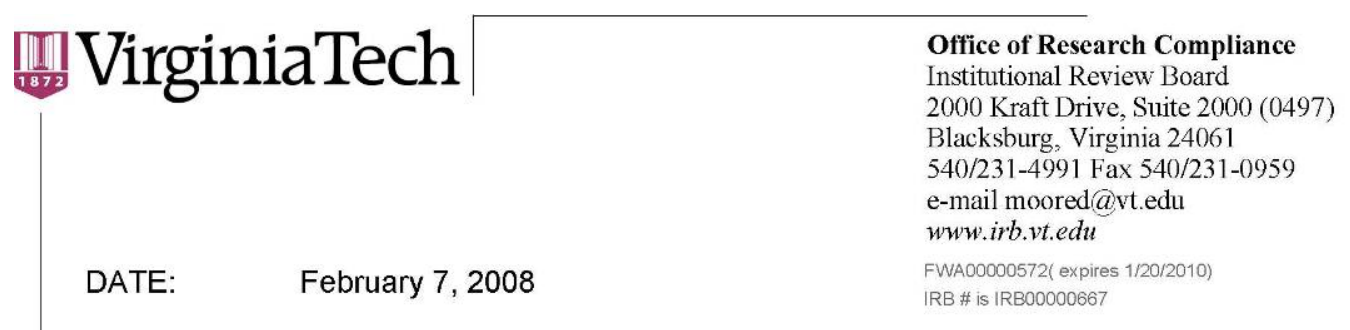

MEMORANDUM

TO:

Gregory Fitch

Jonathan M. Hankey

Grant Compared 2/6/08

Brian M. Kleiner

Approval date: $2 / 7 / 2008$

FROM:

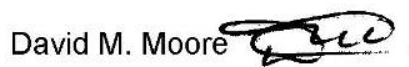

Continuing Review Due Date:1/23/2009

Expiration Date: 2/6/2009

SUBJECT: IRB Expedited Approval: "SAFETEA LU SR - Lighting and Warning System (LWS)", OSP \#415351, IRB \# 08-049

This memo is regarding the above-mentioned protocol. The proposed research is eligible for expedited review according to the specifications authorized by 45 CFR 46.110 and 21 CFR 56.110. As Chair of the Virginia Tech Institutional Review Board, I have granted approval to the study for a period of 12 months, effective February 7, 2008.

As an investigator of human subjects, your responsibilities include the following:

1. Report promptly proposed changes in previously approved human subject research activities to the IRB, including changes to your study forms, procedures and investigators, regardless of how minor. The proposed changes must not be initiated without IRB review and approval, except where necessary to eliminate apparent immediate hazards to the subjects.

2. Report promptly to the IRB any injuries or other unanticipated or adverse events involving risks or harms to human research subjects or others.

3. Report promptly to the IRB of the study's closing (i.e., data collecting and data analysis complete at Virginia Tech). If the study is to continue past the expiration date (listed above), investigators must submit a request for continuing review prior to the continuing review due date (listed above). It is the researcher's

responsibility to obtained re-approval from the IRB before the study's expiration
4. If re-approval is not obtained (unless the study has been reported to the IRB as closed) prior to the expiration date, all activities involving human subjects and data analysis must cease immediately, except where necessary to eliminate apparent immediate hazards to the subjects.

\section{Important:}

If you are conducting federally funded non-exempt research, this approval letter must state that the IRB has compared the OSP grant application and IRB application and found the documents to be consistent. Otherwise, this approval letter is invalid for OSP to release funds. Visit our website at http://www.irb.vt.edu/pages/newstudy.htm\#OSP for further information.

As indicated on the IRB application, this study is receiving federal funds. The approved IRB application has been compared to the OSP proposal listed above and found to be consistent. Funds involving procedures relating to human subjects may be released. Visit our website at www.irb.vt.edu for further information

cc: File

Department Reviewer:Suzanne E. Lee

OSP

VIRGINIA POLYTECHNIC INSTITUTE UNIVERSITY AND STATE UNIVERSITY

An equal opportunity, affirmative action institution 


\section{APPENDIX D - PROTOCOL CAR/ROAD PREPARATION}

- Get Fuel

o Ensure that the test vehicle has at least a half tank of gas in it.

o Ruby should be filled at the motor pool gas station on campus

- A Key Fob is required to get gas from the motor pool

- All receipts must be kept

- Clean Test Vehicle

o Wipe the vehicle's windows down using Windex spray and paper towel.

o Wipe the dashboard, display console, and rear view mirrors as well.

- Cool/Heat Test Vehicle

o Turn the car on and set the AC/Heat on if it's needed.

- Turn DAS On

o Open the trunk (button located on driver door). Turn the DAS on by flipping the two switches up.

o Turn the DGPS unit on (press the black button closest to the trunk opening)

0 Use display in back seat to ensure system is operating.

- $\quad$ Look for DGPS values to flicker

\section{- Emergency Precautions}

o Ensure experimenter has a cell phone and fire extinguisher in the test vehicle.

0 Ensure the fire extinguisher is held in place.

o Ensure Seat Belt Extension is Available for larger participants

- Enter Subject Number in DAS

o In the back seat of the vehicle, there is a computer monitor and keyboard

o Enter Subject Number, Gender, and Age Group

o Look up the Order and select the appropriate "Set".

\section{Go out to Smart Road}

\section{- Turn on Compressor}

1. Make sure the red electrical switch is turned to on (flipped to the down position)

2. Make sure the black fuel line switch is open (toward the compressor)

3. Make sure the red air valve (at the base of the compressor below the electrical switch) it pulled up. This takes the load off the compressor and makes it easier to start)

4. Turn choke on for the first couple of pulls

5. Turn the choke off

6. Pull to start (it should come on then, if not before when the choke is on. It'll depend how cold it is outside) 
7. Once started, fold the red air valve switch down to allow the tank to fill up with air.

- Test Bollards

o Open container by SR

o Press push-button to manually fire bollards

o Ensure each one activated

o Pack them back up by stuffing socks into holes and placing plastic lids back on.

- Setup CD/MP3 player

o Open CD/MP3 display

o Set screen to Tuner

o Close the display 


\section{Experimenter Preparation}

- Check Weather Forecast

o http://www.accuweather.com/us/va/blacksburg/24060/city-weather-

forecast.asp?partner=accuweather\&u=1\&traveler $=0$

0 If it is raining or it is expected to rain during the course of the experiment, call the participant to reschedule.

0 If it is too windy, the experiment will need to be rescheduled.

- Check Voice Mail

o Make sure there aren't any messages from participants calling to cancel/asking for directions

- Check Email

o Make sure there aren't any messages from other staff.

- Determine Participant Number

P:|415355IWorkinglDocuments|Participant OrderlParticipant Order Sheet.xls

- Retrieve Data Sheet

o Retrieve participant data sheet from its respective folder

- Retrieve In-vehicle Experiment Binder

o Retrieve In-vehicle experiment binder containing the pre-experiment instructions, and place the data sheet and post-drive questionnaire in it.

o Go through the participant packet to make sure all the forms are there, and that they have the correct participant number on them.

- In-vehicle Order sheet

- Time in/out

- 2 Informed Consent Forms

- Tax Form

- Anthropometric Data Form

- Post Drive Questionnaires

- In-Vehicle Note sheets

- Wait in lobby for participant 


\section{PARTICIPANT PREPARATION ROOM}

- Set up the Participant Preparation Room

o Close the door

o Turn on all overhead lights

o Get Vision Test Equipment

- Greet Participant

- Record Arrival Time

o Record the time that the participant arrived on the Time-In form

- Get Informed Consent

o Give the participant the form and encourage them to read it

o Answer questions

o Have participant sign and date both forms

o Give the participant a copy of the informed consent

- Ask For Driver's License

o Must be a valid Class A driver's license to proceed with the study

o Out of state is fine

- Confirm that Participant is willing to provide SSN at the end of the experiment. This is required to be paid.

- Give Tax Forms

o To complete the $\mathrm{W}-9$, the participant must fill out the following in the box:

o Name

o Address

- Tax ID number (social security number)

o Sign and date at the bottom

o If the participant makes more than $\$ 500.00$ doing studies from Jan 1 to Dec 31, this will be reported to the IRS as income

o Print the participants name at the top of the reverse side of the tax form. If they question what this is for, you may tell them:

"This form states that we are not hiring you for full time employment. There will not be any health benefits or paid vacation. We can not fire you because we are not hiring you. You can quit at any time without being held liable for services by the University. You are a one-time contractor. If you already work for Virginia Tech, this is completely separate from your job, and your performance will not have any effect on your employment with the university." 


\title{
EXPLAIN PURPOSE OF STUDY
}

\author{
MAKE SURE YOU GIVE THEM THE INFORMED CONSENT FORM, GET \\ THEM TO CHECK THE VIDEO ALLOWANCE CHECK BOXES, AND SIGN IT \\ RECORD TIME IN, GET DRIVER'S LICENSE, AND SSN \\ TURN OFF CELL PHONE AND REMOVE WALLET FROM BACK POCKET
}

I would first like to thank you for agreeing to come out and take part in our Vibrating Seat experiment. Today you will be driving a vehicle around the Virginia Smart Road. The drive will take approximately two hours with several stops for questions. I will be with you in the vehicle at all times.

Cars are now being developed that are capable of alerting drivers to a crash before it occurs. For instance, a forward collision warning system will warn drivers who are about to collide with a vehicle in front of them. A lane change waning system warns drivers about to collide with a vehicle next to them. However, what the best way is to communicate these alerts to drivers is still being determined. The purpose of today's test is to evaluate a vibrating driver seat's ability to communicate crash alerts to drivers. Various vibration patterns will be tested. The vibrations are similar in sensation to a vibrating cell phone. Your task will be to drive the test vehicle at $25 \mathrm{mph}$ down the middle of the Smart Road, experience these vibration alerts, and make the appropriate response, such as pressing on the brakes or steering the vehicle into the left or right lane. I will demonstrate each alert once we are in the vehicle. You will also have time to practice braking or steering in response to the alerts. Once you have made a driving response, your are to also say the type of alert you experienced aloud. You will have time to practice the names of the alerts. Throughout the experiment, I will also ask you to complete questionnaires.

Please be aware that we are testing the vibrating driver seat and not you. The results are not a reflection of your ability. Do you have any questions at this time?

OK, since the vibrating seat will feel different for everyone, I would like to record your height, weight, leg length and hip width to characterize your frame type. We are also required to conduct a vision test. 


\section{VISION TEST}

\section{Protocol:}

1. The participant can wear glasses or contact lenses to meet these criteria but not transition lenses.

2. Attach a Snellen eye chart to a wall in a well lit area that is not too bright. The center of the chart should be positioned at approximately eye-level of the participant (Figure 64). Use a measuring tape to set this up. Tests can be given in any room as long as: (i) there is enough distance to administer the test, (ii) the lighting is consistent for every participant, and (iii) there is no glare on the vision chart that could prevent the participant from accurately viewing the chart.

3. Have the participant stand directly facing the chart with his/her toes on a tape line marked on the floor twenty (20) feet from the wall (Figure 64).

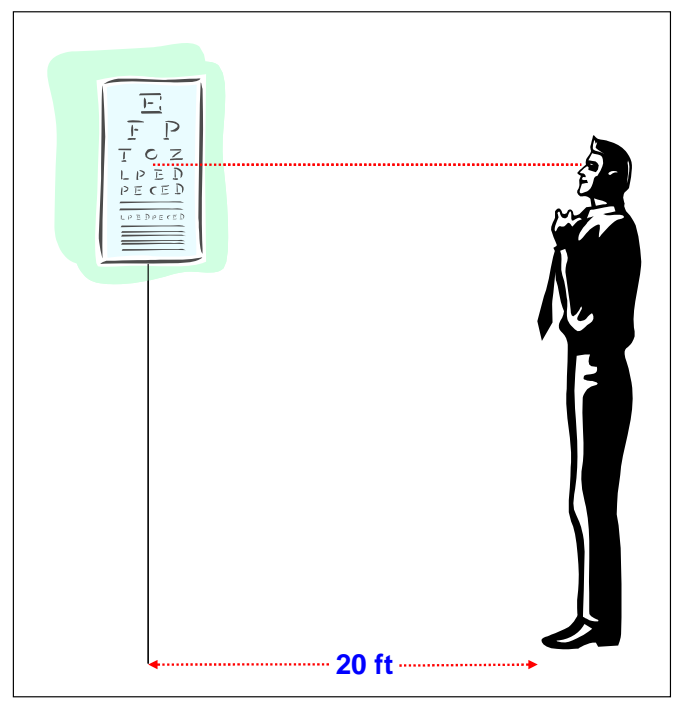

Figure 64. Visual acuity test chart positioning.

4. Sterilize the occluder with an alcohol swab and hand it to the participant.

5. Instruct the participant to not press the occluder on the eye, for it could result in altered vision. The participant should keep both eyes opened (one of them covered).

6. Following the script for the vision test, instruct the participant to look at the wall and read aloud the smallest line that he/she can see. 
7. If the participant gets every letter on that line correct, have him/her read the nextine down in the same manner. Continue this process until the participant can no longer read an entire line correctly. Record the visual acuity of the last completed line.

8. If the participant did not get every letter correct in the first line read, have him/her read the line above in the same manner. Repeat as necessary until a line is read correctly. Record the visual acuity of the first completed line.

9. Repeat the entire process for the other eye, and record the visual acuity for that eye.

\section{Script for Visual Acuity Test:}

We will now perform an informal vision test. You should wear your corrective glasses or contact lenses for this test. Please stand with your toes on the tape that you see on the floor, and face the eye chart ahead. Use this occluder to cover one eye at a time. Please do not press the occluder against your eye. Keeping both eyes open, place it over your left eye, and using only your right eye, read aloud the smallest line that you can see.

If the line is read successfully, read:

Please read the line below that.

Repeat until a line is missed, then record the vision number from the line above.

If the line is not read successfully, read:

Please read the line above that one.

Repeat until one full line is read correctly, and record the vision number from that line.

Now, give your eyes as long as they need to rest and refocus. When you are ready, keeping both eyes open, place the occluder over your right eye, and read aloud the smallest line that you can see using only your left eye.

If the line is read successfully, read:

Please read the line below that.

Repeat until a line is missed, then record the vision number from the line above.

If the line is not read successfully, read:

Please read the line above that one.

Repeat until one full line is read correctly and record the vision number from that line. 
If the participant has a visual acuity of $20 / 40$ or better, record the visual acuity of the last completed line.

Good. You have good sight for this experiment.

If the participant does not have a visual acuity of 20/40 or better, record the visual acuity of the last completed line and explain that he/she is not eligible for this research project.

Unfortunately you are not eligible for this particular study as you may have trouble seeing the text messages on the test device in our experiment. Thank you for your time.

We will compensate you for your time. Would you like to be called for future studies?

\section{HEIGHT, WEIGHT, AND LEG LENGTH AND WIDTH}

1. Place the scale on a hard surface (non-carpeted).

2. Ask the participant to push any of the five buttons on the front of the scale with their toe.

3. Once the scale reads " 0.0 ", ask the participant to step on the scale.

4. Once the participant's weight is displayed, record the participant's weight on the participant screening data sheet.

5. Ask the participant to step off the scale.

6. Ask the participant to remove their shoes and step onto the base of the height meter.

7. Place the sliding height measurer at the top of the participants head.

8. Record the participant's height on the participant screening data sheet.

9. Ask participant to sit on test chair

10. Using a ruler, measure distance across both thighs

11. Using ruler, measure distance from their back to the front of their knees.

\section{Before you Leave}


We are now ready to head out to the Road. Do you need to use the restroom?

Take participant to the experimental vehicle parked outside the front door. 


\section{IN VEHICLE ORIENTATION}

Put On Seatbelt

Go ahead and buckle your seat belt.

Occupant position

Okay, now we are going to have you adjust your seat. The seat controls are on the side of the seat near the door. You can adjust up down, forward backward and the seat back recline.

Adjust Steering wheel

There is a control also to adjust the steering wheel on the left side of the steering column.

Adjust Mirrors

The controls to adjust the side mirrors are here. Once I close the door, please adjust the side and rear view mirrors.

Once participant is in place, get in the back right of the test vehicle and put your seatbelt on

Participant Comfort Check

Okay, are you comfortable? 


\title{
MAKE SURE YOU PRESS
}

\author{
SHIFT + S
}

\section{TO RECORD THE DATA}




\section{EXPERIMENT PART I}

\section{Please take your wallet out of your pocket and turn your cell phone off.}

Today, you are to drive a vehicle at $20 \mathrm{mph}$ down the middle of a two-lane road. Your right tire will be in the right lane, and your left tire will be in the left lane. As you drive, the driver seat will vibrate in different ways. Each vibration, referred to as an alert, requires a specific driving response, such as stopping or steering the car. After performing the response, you must say the name of the alert. Please note that I will be controlling when the alerts are generated.

I will now explain the vibrating driver seat alerts. The driver seat can vibrate in seven different ways to show seven different alerts. Each alert is designed to warn you of a specific type of crash.

Three of the seven alerts are designed to warn you of trouble ahead. This is done by vibrating the front edge of the driver seat.

The first alert is a "Stopping Car" Warning which is intended to warn you of a stopping car. This alert consists of five vibration pulses along the front edge of the seat. I will now play this alert so you can feel it.

$<$ Play the FCW alert. Open Set 6 in SOL and use the $n$ and $p$ keys to scroll to the appropriate alert. Hit t to play the alert.>

When you experience this alert, you are to press the brake pedal as fast as possible and slow the vehicle in a safe and controlled manner. Imagine you are stopping to a braking lead vehicle. After you have slowed the car down, you are to say "Stopping Car."

The second alert is a Sharp Turn Warning which is intended to warn you when you are coming up on a sharp turn. This alert consists of one long vibration along the front edge of the seat. I will now play this alert so you can feel it.

$<$ Play the CSW alert. Again, using Set 6, advance to the CSW alert and hit t to play it.>

When you experience this alert, you are to press the brake pedal as fast as possible and slow the vehicle in a safe and controlled manner. Imagine you are stopping to avoid a sharp turn. After you have slowed the car down, you are to say "Curve Ahead."

The third alert is a Red Light Warning, which is intended to warn you when you are about to run a red light. This alert consists of two double pulses along the front edge of the seat. I will now play this alert so you can feel it.

\section{$<$ Play the IVW alert in Set 6>}

When you experience this alert, you are to press the brake pedal as fast as possible and slow the vehicle in a safe and controlled manner. Imagine you are stopping to avoid running a red light. After you have slowed the car down, you are to say "Red Light". 
To summarize these last three alerts, any vibration along the front of the driver seat is intended to warn you of danger ahead. The correct driving response is to press the brakes as fast as possible and slow the car down. When you feel five pulses, say "Car Ahead". When you feel one long vibration, say "Sharp Turn". When you feel two double pulses, say "Red Light".

The next four alerts are left and right versions of two types of warnings.

The fourth alert is a "Car Left" Warning which is intended to warn you when you are about to hit a car on your left side. This alert consists of five vibration pulses in the back left corner of the driver seat. I will now play this alert so you can feel it.

\section{$<$ Play the Left LCW alert. Find the LCWL alert in Set 6 and play it. $>$}

When you experience this alert, you are to steer the vehicle into the right lane as fast as possible. Pretend you are steering back into a lane after nearly hitting a vehicle when making a left lane change. After you have steered right, you are to say "Car left".

The fifth alert is a Car Right Warning which is intended to warn you when you are about to hit a car on your right. This alert consists of five vibration pulses in the back right corner of the driver seat. I will now play this alert so you can feel it.

\section{$<$ Play the Right LCW alert. Find the LCWR alert in Set 6 and play it. $>$}

When you experience this alert, you are to steer the vehicle into the left lane as fast as possible. Pretend you are steering back into a lane after nearly hitting a vehicle when making a right lane change. After you have steered left, you are to say "Car right".

The sixth alert is a "Drift Left" Warning which is intended to warn you when you drift over to the left lane. This alert consists of one long vibration in the back left corner of the driver seat. I will now play this alert so you can feel it.

\section{$<$ Play the Left LDW alert. Find the LDWL alert in Set 6 and play it.>}

When you experience this alert, you are to steer the vehicle into the right lane as fast as possible. Pretend you are steering back into your lane after drifting out of it. After you have steered right, you are to say "drift left."

The final alert is a "Drift Right" Warning which is intended to warn you when you drift over to the right lane. This alert consists of one long vibration in the back right corner of the driver seat. I will now play this alert so you can feel it.

\section{$<$ Play the Right LDW alert. Find the LDWR alert in Set 6 and play it. $>$}

When you experience this alert, you are to steer the vehicle into the left lane as fast as possible. Pretend you are steering back into your lane after drifting out of it. After you have steered left, you are to say "drift right."

To summarize these last four alerts, vibrations in the back left corner of the driver seat are intended to warn you of danger on the left. The correct driving response is to steer into the right lane as fast as possible.

Vibrations in the back right corner of the driver seat are intended to warn you of danger on the right. The correct driving response is to steer into the left lane as fast as possible. When you feel five pulses, say "Car left, or Car right". When you feel one long vibration, say "Drift Left" or "Drift Right".

As a hint, any vibration that has five pulses in it is intended to warn you about a car. So, five pulses on the front of the seat is a stopping car warning, five pulses on the left is a car left warning, and five pulses on the right is a car right warning. 
Do you have any questions at this time?

Ok, please drive the car over out of the parking lot over to the Smart Road.

IF YOU HA VEN'T DONE IT YET,

\title{
MAKE SURE YOU PRESS
}

\author{
SHIFT + S
}

\section{TO RECORD THE DATA}




\section{Once on the Road}

Radio Dispatch to let them know you want to get on the road. Say "Hi Dispatch, do you copy?" What for a reply. Then say, "This is for the LWS study 415355. We are in the red sedan and would like to get on the road.

As you loop around the corner, say the following:

Please remember that you are to drive at $20 \mathrm{mph}$ for this study. Please keep your foot on the gas pedal when you are not responding to the alerts. Since there is a hill on this road, please place the car in $1^{\text {st }}$ gear when going down it to prevent the car from speeding up while your foot is on the gas pedal. When going down the steeper parts of the hill, you can drive at $17 \mathrm{mph}$ to keep the engine RPMs below 2500 to save gas. When going up the hill, please use the normal drive gear.

Once you get around the loop, say the following:

Ok, please place the car in first gear and move the car to the middle of the road.

Ok, we are now going to practice before we start the experiment.

Set 1 - FCW Only

Ok, for this part of this experiment, I'm only going to present the stopping car warning. When you feel this alert, 5 pulses along the front edge of the seat, slow the car and say "Stopping Car". Please remember to perform the response as fast as possible. After you perform the response, please say the name of the alert. I'm going to give you some time to practice before we begin.

\section{PRACTICE}

If they make a mistake in manual or verbal response, correct them. Instruct them to continue driving at 20 mph after slowing the car down.

\section{Run Trials}

Do not correct their mistakes.

After Set 1, give them the Number of Alerts Questionnaire (Set 1) questionnaire. I have a questionnaire for you to fill out.

<Give the questionnaire. Make sure they answered each question>

\section{Set 2 - FCW, LCW L, LCW R}

Ok, for this part of this experiment, I'm going to present the stopping car warning, the car left warning, or the car right warning. All three warnings consist of 5 vibration pulses. When you feel the stopping car warning, slow the car down. When you feel the car left warning, steer right. When you feel the car right warning, steer left. Please remember to perform the response as fast as possible. After you perform the response, please say the name of the alert. I'm going to give you some time to practice before we begin.

\section{PRACTICE}

If they make a mistake in manual or verbal response, correct them. Instruct them to continue driving at 20 mph after slowing the car down. Instruct them to return to the center lane after steering into an adjacent lane.

\section{Run Trials}

Do not correct their mistakes.

\section{After Set 2, give them the Number of Alerts Questionnaire (Set 2) questionnaire.}


I have a questionnaire for you to fill out.

<Give the questionnaire. Make sure they answered each question>

\section{Set 3 - FCW, CSW, IVW, LCW L, LCW R, LDW L, LDW R}

Ok, for this part of this experiment, I'm going to present all seven alerts. When you feel the stopping car warning, sharp turn warning, or red light warning, slow the car down. Remember that all three consist of vibrations along the front of the seat. When you feel the car left warning, or drift left warning, you are to steer right. Remember that both warnings consist of vibrations in the back left of the seat. When you feel the car right warning, or drift right warning, please steer left. Remember that both warnings are in the back right of the seat.

As a hint, the alerts that indicate a car, such as the stopping car, car left, and car right warnings, consist of five vibration pulses. Another hint is that the drift left and drift right warnings, which are played in the back corners of the driver seat, try to resemble driving over a rumble strip by using one long vibration.

Please remember to perform the appropriate driving response as fast as possible. After you perform the response, please say the name of the alert. I'm going to give you some time to practice before we begin.

\section{PRACTICE}

If they make a mistake in manual or verbal response, correct them. Instruct them to continue driving at 25 mph after slowing the car down. Instruct them to return to the center lane after steering into an adjacent lane.

\section{Run Trials}

Do not correct their mistakes.

After Set 3, give them the Number of Alerts Questionnaire (Set 3) questionnaire. I have a questionnaire for you to fill out.

<Give the questionnaire. Make sure they answered each question>

After all Sets, give them the Number of Alerts Questionnaire (Overall) questionnaire. I have a questionnaire for you to fill out. 


\section{EXPERIMENT PART II}

$<$ Check the participant order to see if they get both alerts in the seat pan first (Set 4), or alerts in the seat pan and seat back first (Set 5)>

For the next part of the experiment, your task will be to drive the vehicle at $20 \mathrm{mph}$ down the left or right lane and make lane changes into the opposite lane. I will instruct you which lane to drive in. As you perform lane changes, you may feel an alert. For instance, when making a left lane change, you may experience a "drift left" warning, a "car left" warning, or no warning at all.

However, unlike the previous tasks, you are only to steer right when you feel the "car left" warning. (Pause)

Therefore, you are to continue the lane change into the left lane if you feel the "drift left" warning, or no warning at all. Recall, that the "car left" warning is 5 pulses in left back left corner of the seat, while the "drift left" warning is a continuous vibration in the back left of the seat. The same goes for when you make right lane changes. Only steer back into the left lane when you experience a "car right" warning.

You do not have to say the name of the alert for this part of the experiment, just perform the lane changes. To represent real life lane changes, please use the turn signals, check your mirrors, and check your blind spots when making the lane changes.

Do you have any questions? OK, please place the car in drive and proceed down the right lane.

\section{If Set 4}

PRACTICE

$<$ If they make a mistake in manual response, correct them. Have them steer in the appropriate lane.

- If a LCWL or LDWL is to be presented, they should be in the right lane and making a left lane change

- If a LCWR or LDWR is to be presented, they should be in the left lane and making a right lane change>

\section{Run Trials}

Do not correct their mistakes.

\section{After Set 4, give them the Haptic Alert Location Questionnaire (Set 4) questionnaire.}

I have a questionnaire for you to fill out.

<Give the questionnaire. Make sure they answered each question>

\section{Set 5}

For this part of the experiment, the "drift left" and "drift right" warnings will be presented through the bottom of the seat. As stated earlier, you are to ignore these warnings. The "car left" and "car right" warnings, however, will be presented by vibrating the upper back of the seat by your spine. You are to steer back into the original lane when you feel these vibrations. 
As a hint, ignore the alerts played through the bottom of the seat and continue the lane change. Steer away when you feel the alerts played through the upper back of the seat.

Do you have any questions at this time?

\section{PRACTICE}

$<$ If they make a mistake in manual response, correct them. >

\section{Run Trials}

Do not correct their mistakes.

After Set 5, give them the Haptic Alert Location Questionnaire (Set 5) questionnaire.

I have a questionnaire for you to fill out.

<Give the questionnaire. Make sure they answered each question>

After Set 4 and 5, give them the Haptic Alert Location Questionnaire (Overall) questionnaire.

Have the participant drive the car to the bottom of the Smart Road. Have them stop the car. 


\section{EXPERIMENT PART III}

\section{Preparation}

Make sure the player is in the CD/MP3 mode. Make sure the volume is off.

The next part of the study involves operating a Clarion CD/MP3 player as you drive.

$<$ Get out of the car and open the Clarion CD/MP3 player touch screen. Show participant were it is. Sit back in the seat when done.>

The Clarion CD/MP3 player is a touch screen display that allows drivers to select and play MP3s by pressing icons with their finger. You are to drive the vehicle at $2 \mathrm{mph}$ up the right lane. While you drive, I will ask you look for a specific icon and press it with your finger. You are to only perform the requested tasks after I say the word "Go". Please try to perform the task as fast as possible. Also, please do not look at the display before I say this command.

Lets practice this task before we begin the experiment. When I say "Go", could you please press the icon that says "Tuner" with your finger?

Ok, GO!

$<$ Wait for participant to press tuner icon $>$

After I say "GO", please press "tuner" in the top left of the screen. This will return you to the main menu.

Ok, GO!

$<$ Wait for them to press "Tuner" in the top left of the screen. They should return to the main menu. >

Ok, great. Please place the car in drive and proceed to drive up the Road.

We're now going to start the experiment. When I say "Go", please press the icon that says "DVD"

$\mathrm{Ok}, \mathrm{GO}$ !

$<$ Wait for participant to press DVD icon $>$

After I say "GO", please press the "DVD" icon in the top left of the screen. This will return you to the main menu.

$\mathrm{Ok}, \mathrm{GO}$ !

< They should return to the main menu. >

When I say "Go", could you please press the icon that says "Tuner" with your finger? 
$\mathrm{Ok}, \mathrm{GO}$ !

<Wait for participant to press tuner icon>

After I say "GO", please press the "Tuner" icon in the top left of the screen.

$\mathrm{Ok}, \mathrm{GO}$ !

< They should return to the main menu. >

When I say "Go", could you please press the icon that says "Sirius" with your finger?

$\mathrm{Ok}, \mathrm{GO}$ !

$<$ Wait for participant to press tuner icon>

After I say "GO", please press the "Sirius" icon in the top left of the screen.

Ok, GO!

< They should return to the main menu. >

When I say "Go", could you please press the icon that says "DVD" with your finger?

$\mathrm{Ok}, \mathrm{GO}$ !

$<$ Wait for participant to press DVD icon>

\section{SURPRISE CONDITION}

When I say Go, please press the icon that says "Settings"

$<$ In order to get the bollards up before the car drives over them, you should aim to launch them as the car enters the intersection. You should therefore, say GO as the car enters the intersection

After you say GO, and the driver looks down at the display, you are to activate the bollards as soon as they look away. Check the participant order to see if this participant gets the FCW warning at the same time.

- Make sure the bollards are enabled.

- Make sure the FCW is enabled if they're getting the Alert

- Make sure loop has been incremented (from 0 to 1 for example)

- Press " $t$ " to launch the bollards and the alert together

- Press "f" to launch just the bollards without the FCW alert>

$\mathrm{Ok}, \mathrm{GO}$ !

<Press the bollard launch button. After the car comes to a complete stop, say the following:> 


\section{A1. If they didn't get the alert, say this}

I apologize for not being able to tell you the additional purpose of this research project prior to data collection. We were interested in measuring how long it would take to see the inflatable barricade in the road. If you have further questions about the study, I can answer them at this time. All known precautions were taken to ensure your complete safety today. Please be aware that how you responded to this event is not an indication of your driving abilities. You should not be worried about your driving abilities on open public road as a result of this test.

\section{A2. If they got the alert, say this}

I apologize for not being able to tell you the additional purpose of this research project prior to data collection. This research is also evaluating the vibrating seat's ability to help drivers detect obstacles in the road. All known precautions were taken to ensure your complete safety today. Please be aware that how you responded to this event is not an indication of your driving abilities. You should not be worried about your driving abilities on open public road as a result of this test.

\section{B1. If They Drive Over the Barricade}

Please realize that the timing of the inflatable barricade was set to be very short. Your ability to avoid the barricade may have been compromised by factors outside of your control, such as unfamiliarity with this vehicle and inexperience with performing braking with this vehicle. Therefore, if you ran over the barrier you should not be concerned. It is not an indication of your driving ability.

\section{C1. Read to All}

We ask that you do not talk about the details of this study to others after your participation because this may invalidate future data that may be collected. I have one questionnaire to give you. After that, the experiment is over. I assure you that there will be no more unexpected events.

We again assure you that all data will be treated with complete confidentiality. Shortly after participating, your name will be separated from the data. A coding scheme will be employed to identify the data by subject number only (for example, Subject No. 7). It is your right to request that your data be deleted. If you would like your data to not be used, please inform the experimenter and indicate your selection on the following page.

<Give the questionnaire. Make sure they answered each question>

If the bollards didn't go up.

I'm sorry, I keep getting the settings button mixed up. You were suppose to press the list button. We'll have to try that again. Please continue around the loop.

Continue to drive around the loop. Pretend nothing went wrong. Have them perform another touch screen task on the way down the road. Have them press the list button when you say go. Afterwards, pretend you need to adjust a setting on the camera. Have them pull into turn around 2 . 
Ok, please pull into the turn around here on the right. I am going to adjust the focus of the camera so that it points at the touch screen display. This will just take a minute.

Review the computer settings to launch the bollards.

Check bollards were enabled

Check loop number was incremented

Have them drive up the road. Have them look for the "menu" button as you launch the bollards.

If the bollards still don't launch. Say that the camera just stopped recording and you need to have someone fix it. Call Greg Fitch. He will come out and get in the car.

Say that a camera error message popped up and you don’t know how to fix it.

Please wait at the entrance of the SR so the participant doesn't associate the problem with the intersection.

Press Q to quit and save the program when done. 
Back at VTTI

\section{Shut System Down}

- $\quad$ Press $Q$ to quit and save data>

\section{Pay the participant}

Document Time on Participant's Debriefing Sheet and give them the sheet.

Pay the participant $\$ 20.00$ per hour, rounding up to the next half hour if necessary.

Make sure the participant signs the payment log.

\section{Transfer Data}

- The HD should be brought to Greg Fitch for data upload.

\section{Review Post-Drive Questionnaire}

- Go over it with them for meaning and legibility. Clearly identify your notes, keep separate from theirs.

Record time for payment

- Fill out the receipt form

\section{Verify Participant's File}

- Verify that the participant's file is complete with questionnaires, interview, and video, and that the data file is backed up on the server.

\section{Clean up the participant preparation room}


Participant \#:

\section{APPENDIX E - NUMBER OF ALERTS QUESTIONNAIRE}

Please answer each question by selecting a number on the scale that best reflects your response. Half numbers, such as 4.5, are also acceptable. Please write down the number on the blank response line.

1. Please rate how difficult it was to make the correct driving action for each vibration alert.

Response:

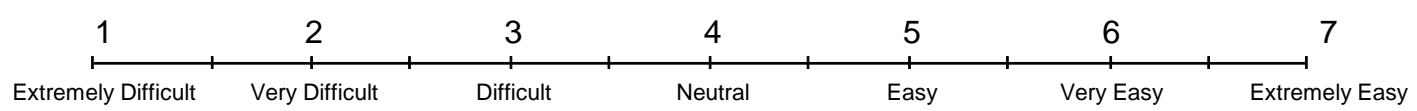

2. Please rate how difficult it was to identify the vibration alerts.

Response:

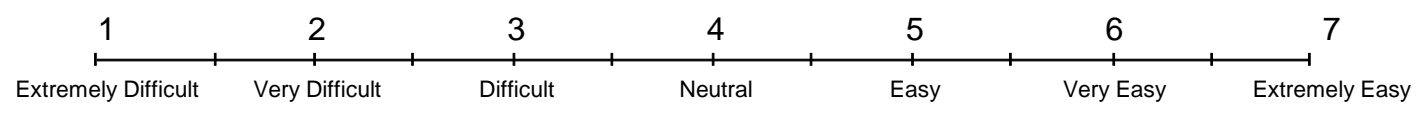

3. Please rate how confusing the vibration alerts were.

Response:

\begin{tabular}{|c|c|c|c|c|c|c|}
\hline 1 & 2 & 3 & 4 & 5 & 6 & 7 \\
\hline $\begin{array}{c}\text { Extremely } \\
\text { Understandabble }\end{array}$ & $\begin{array}{c}\text { Very } \\
\text { Understandable }\end{array}$ & & & & & \\
\hline
\end{tabular}

4. Please rate how distinct the vibration alerts were from each other.

Response:

\begin{tabular}{|c|c|c|c|c|c|c|}
\hline 1 & 2 & 3 & 4 & 5 & 6 & 7 \\
\hline Extremely Blurry & Very Blurry & Blurry & Neutral & Well-Defined & $\begin{array}{c}\text { Very } \\
\text { Well-Defined }\end{array}$ & $\begin{array}{c}\text { Extremely } \\
\text { Well-Defined }\end{array}$ \\
\hline
\end{tabular}


Participant \#:

5. Please rate how difficult it was to perform this task.

Response:

\begin{tabular}{|c|c|c|c|c|c|c|}
\hline 1 & 2 & 3 & 4 & 5 & 6 & 7 \\
\hline Extremely Difficult & Very Difficult & Difficult & Neutral & Easy & Very Easy & Extremely Easy \\
\hline
\end{tabular}

6. How appropriate is it for the driver seat to vibrate like this when intended to alert you to a crash threat?

Response:

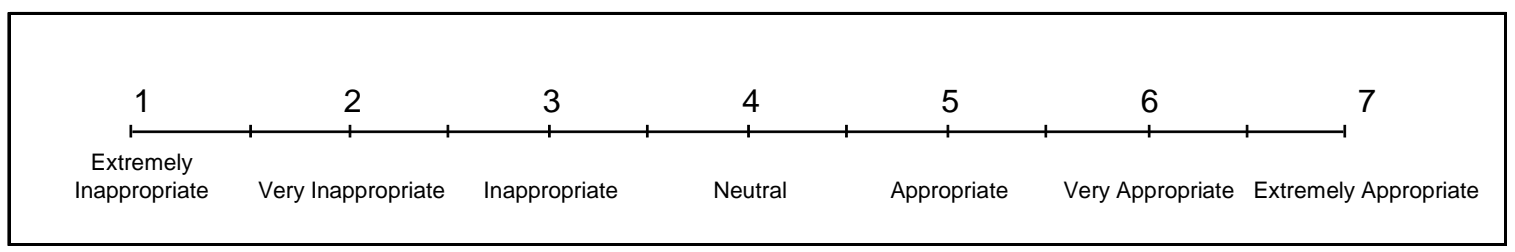


Participant \#:

\section{APPENDIX F - NUMBER OF ALERTS QUESTIONNAIRE (OVERALL)}

Please answer each question by selecting a number on the scale that best reflects your response. Half numbers, such as 4.5, are also acceptable. Please write down the number on the blank response line.

1. Please rate how urgent the vibration alerts were.

Response:

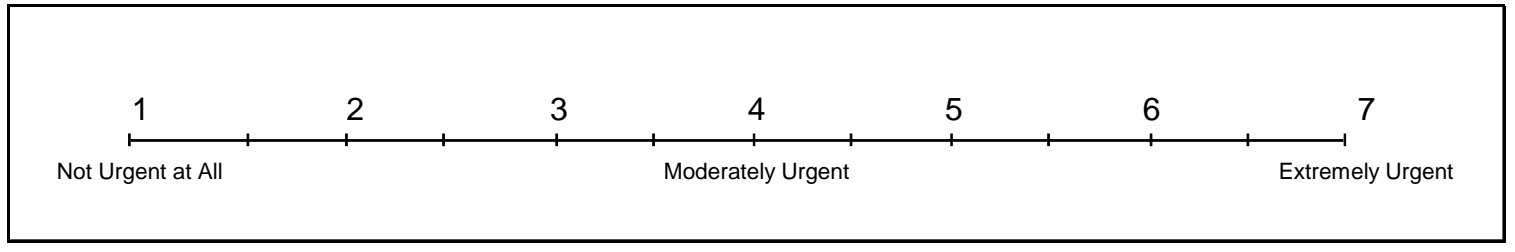

2. Please rate how annoying the vibration alerts were.

Response:

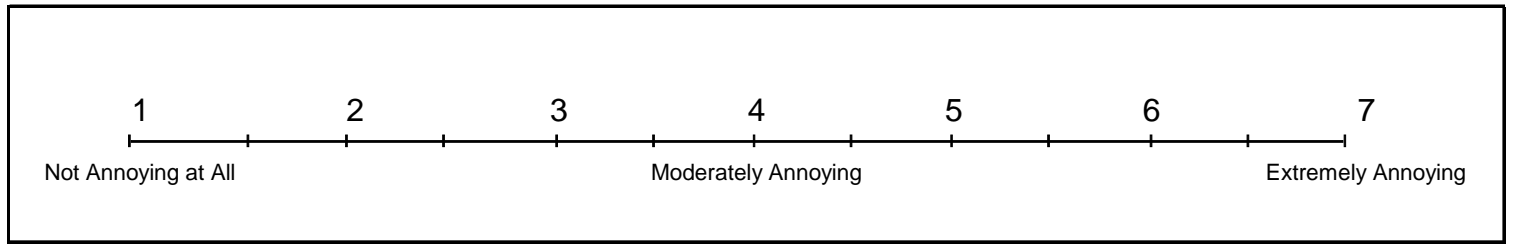

3. Please rate the intensity of the vibration alerts.

Response:

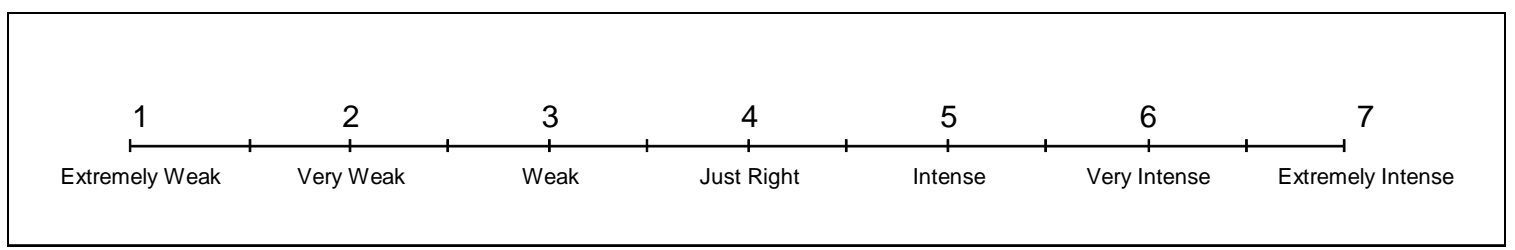

4. Please rate the duration of the vibration alerts.

Response:

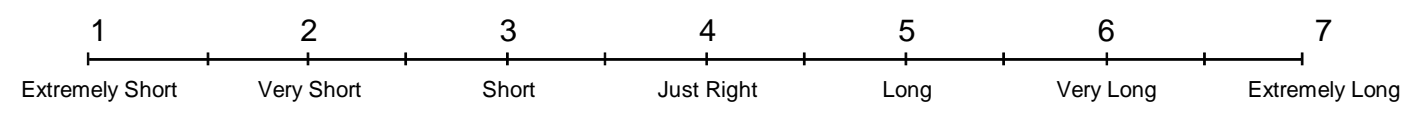




\section{APPENDIX G - REMOVAL OF RESPONSE TIME OUTLIERS}

Figure 65 shows a scatter plot of the observed practical manual response times for each condition in Experiments 1 and 2. With respect to Experiment 1, no data were removed from the "1 Alert" distribution. Outliers in the remaining conditions were removed as follows. The longest response time in the " 3 Alert" condition was removed (leaving response times shorter than or equal to $2599 \mathrm{~ms}$ ). The longest response time in the "7 Alert" condition was removed (leaving response times shorter than or equal to $4300 \mathrm{~ms}$ ). With respect to Experiment 2, no data were removed from the "Seat Pan" condition. The longest response time in the "Seat Pan + Seat Back" condition was removed (leaving response times shorter than or equal to $2133 \mathrm{~ms}$ ). The removed data are highlighted below with red circles.

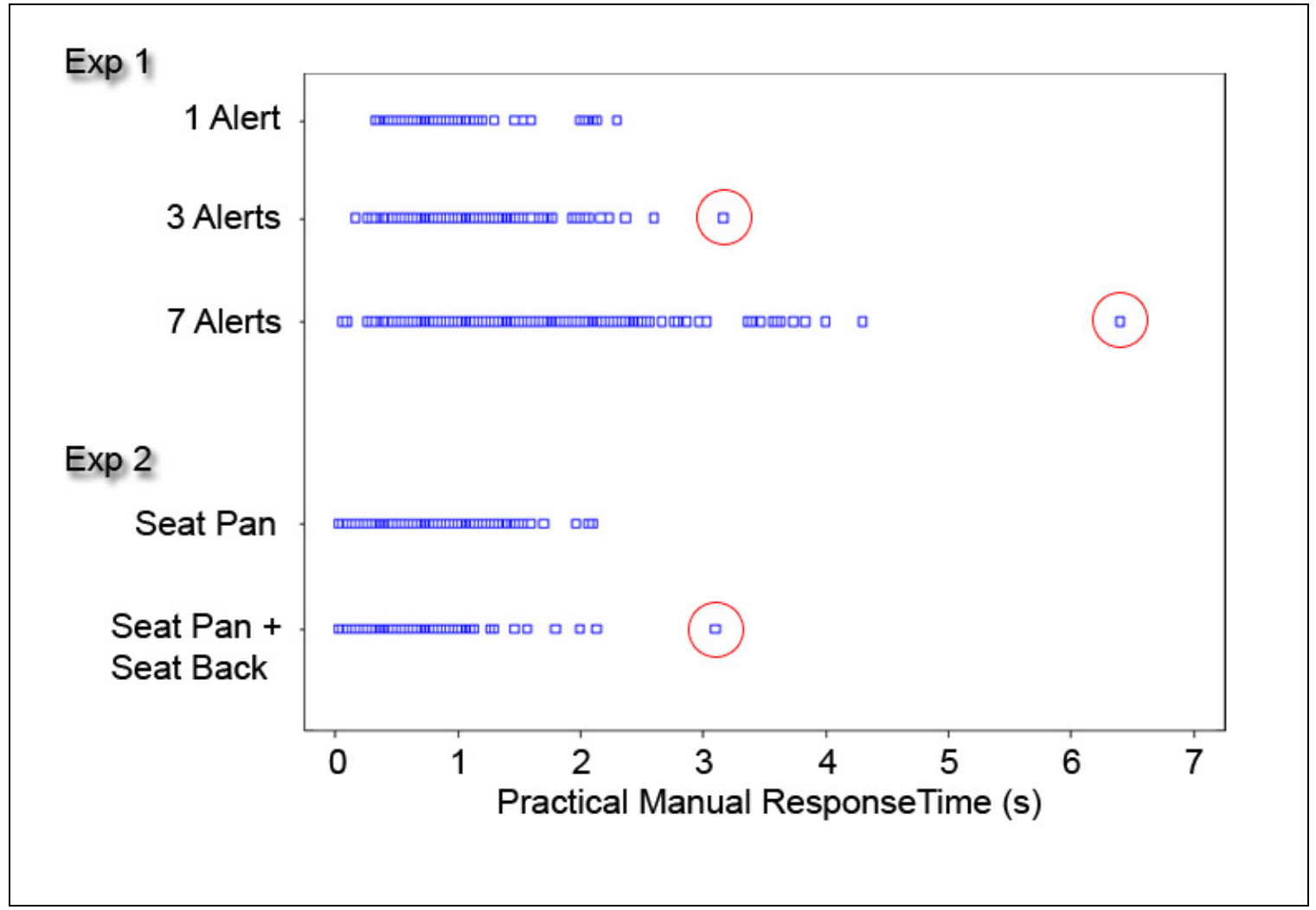

Figure 65. Scatter plot of the observed practical manual response times for each experimental condition. 


\section{APPENDIX H - ADDITIONAL DRIVER RATINGS OF ALERTS IN EXPERIMENT 1}

At the end of Experiment 1, participants answered general questions regarding the alerts they experienced. The results are presented in this appendix.

Question 1: Please rate how urgent the vibration alerts were.

On average, drivers found the haptic driver seat alerts to be between moderately to extremely urgent (mean response $=5.125$, s.e. $=0.23$ ). Figure 66 shows drivers' ratings broken down by age and gender.

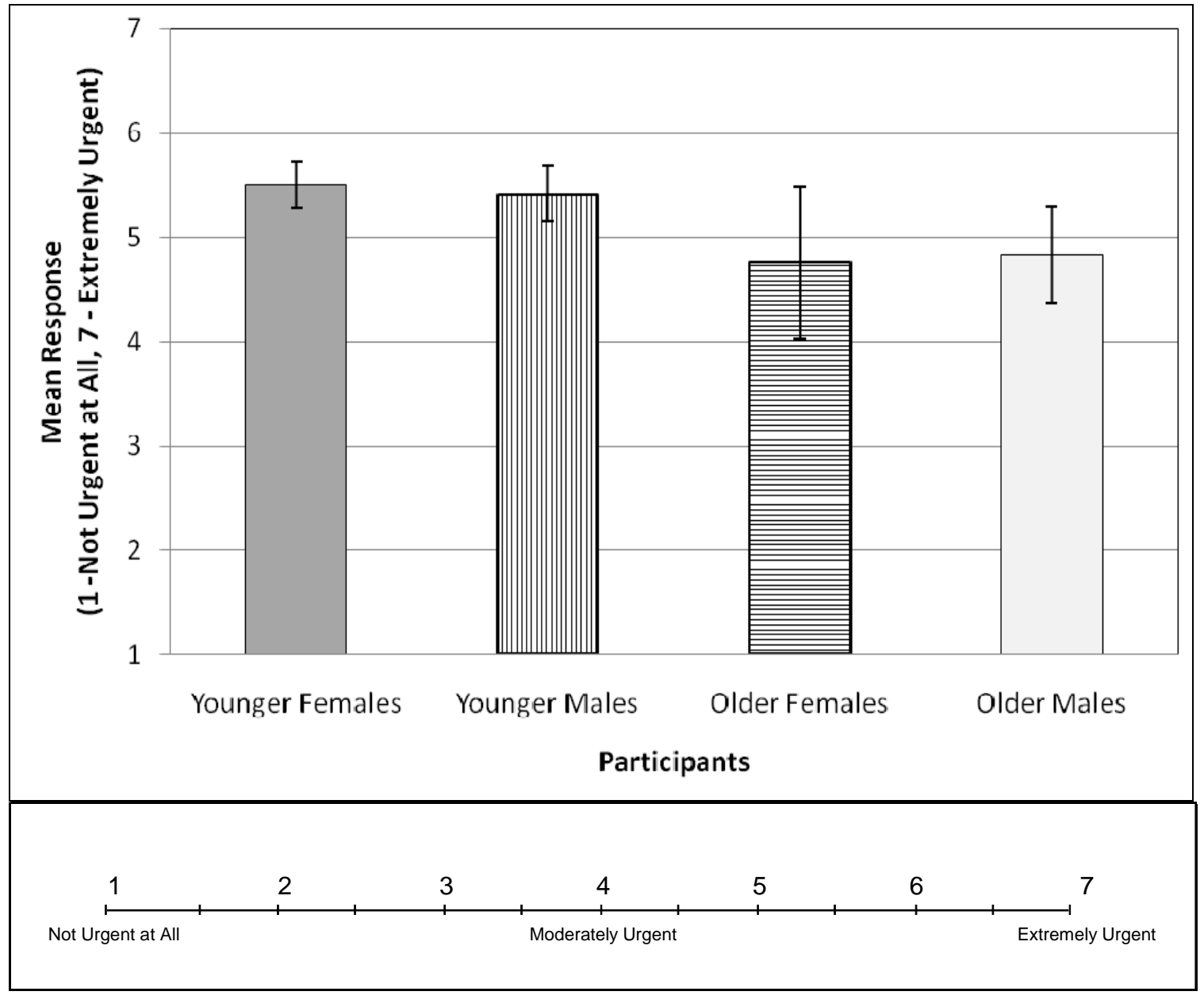

Figure 66. Drivers' mean ratings of how urgent the alerts were. 
Question 2: Please rate how annoying the vibration alerts were.

On average, drivers found the haptic driver seat alerts to be less than moderately annoying (mean response $=2.85$, s.e. $=0.31$ ). Figure 67 shows drivers' ratings broken down by age and gender. It is interesting that younger males appeared to have rated the alerts to be more annoying than the other participants.

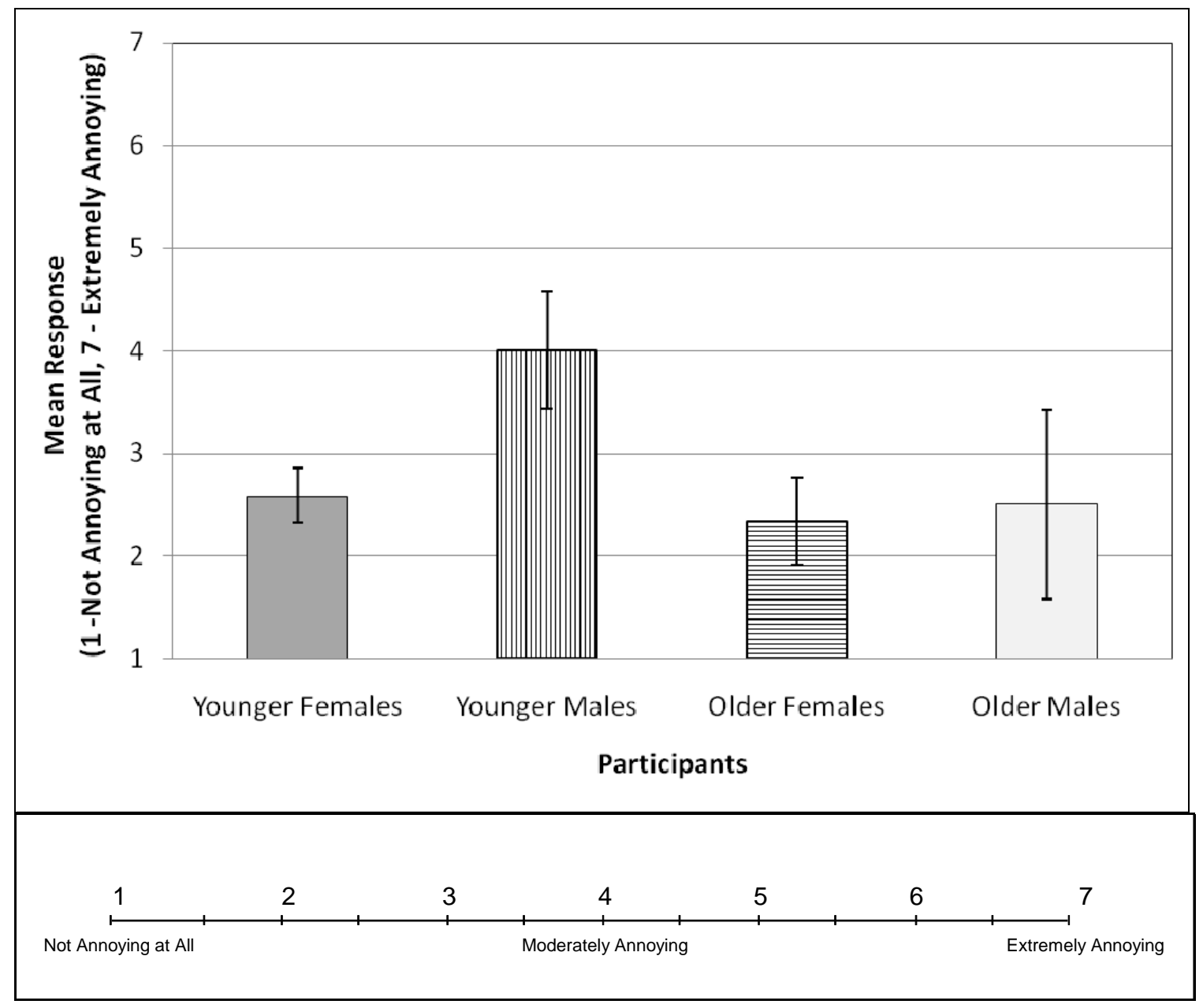

Figure 67. Drivers' mean ratings of how annoying the alerts were. 
Question 3: Please rate the intensity of the vibration alerts.

On average, drivers found the haptic driver seat alerts to be intense (mean response $=$ 4.54 , s.e. $=0.21$ ). Figure 68 shows drivers' ratings broken down by age and gender. Younger males were observed to rate the alerts as more intense than the other participants. This may be why they rated the alerts as more annoying relative to the other participants.

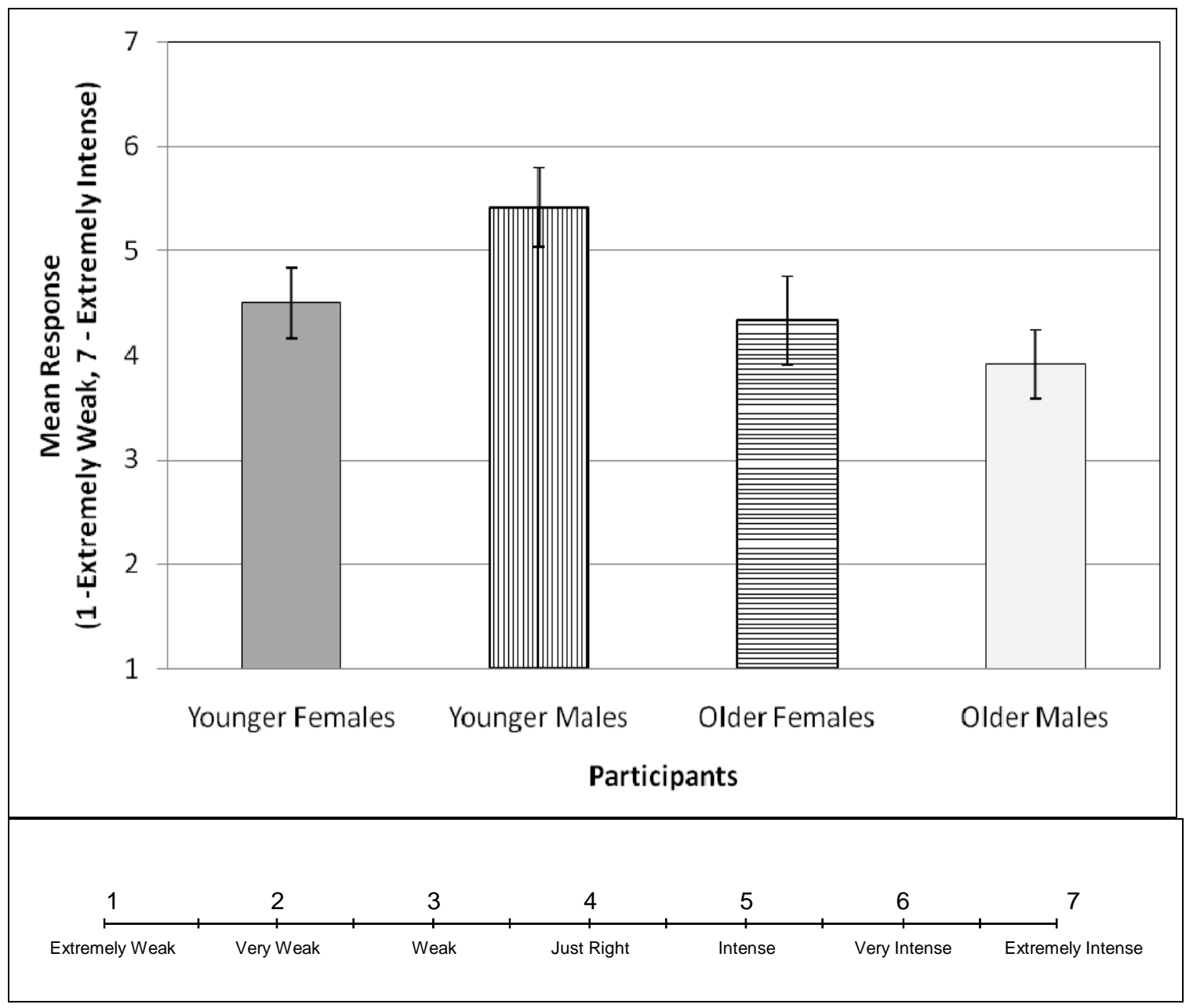

Figure 68. Drivers' mean ratings of how intense the alerts were. 
Question 4: Please rate the duration of the vibration alerts.

On average, drivers found the haptic driver seat alerts to be just right (mean response $=$ 4.00 , s.e. $=0.79$ ). Figure 69 shows drivers' ratings broken down by age and gender.

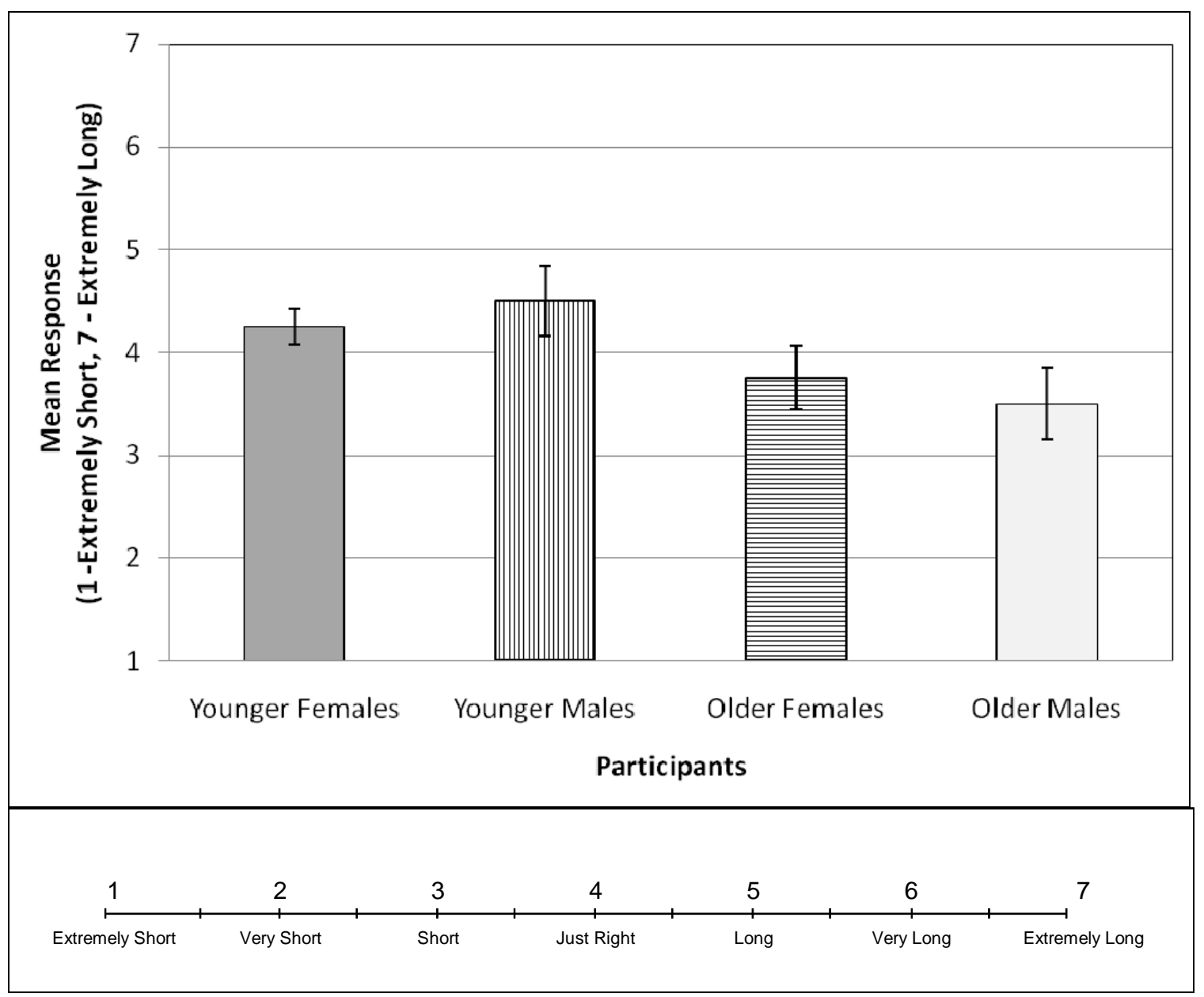

Figure 69. Drivers' mean ratings of how long the alerts were. 
Participant \#:

\section{APPENDIX I - HAPTIC ALERT LOCATION QUESTIONNAIRE}

Please answer each question by selecting a number on the scale that best reflects your response. Half numbers, such as 4.5, are also acceptable. Please write down the number on the blank response line.

1. Please rate how easy it was to distinguish meaningful vibration alerts from those you were not supposed to respond to.

Response:

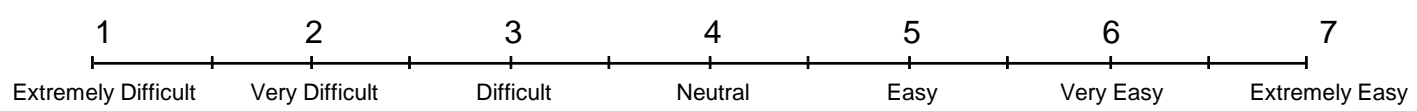

2. Please rate how distinct the vibration alerts were from each other.

Response:

\begin{tabular}{|c|c|c|c|c|c|c|}
\hline 1 & 2 & 3 & 4 & 5 & 6 & 7 \\
\hline Extremely Blurry & Very Blurry & Blurry & Neutral & Well-Defined & $\begin{array}{c}\text { Very } \\
\text { Well-Defined }\end{array}$ & $\begin{array}{c}\text { Extremely } \\
\text { Well-Defined }\end{array}$ \\
\hline
\end{tabular}

3. Please rate how difficult it was to perform this task.

Response:

\begin{tabular}{llcrrrr}
1 & 2 & 3 & 4 & 5 & 6 & 7 \\
\hline Extremely Difficult & Very Difficult & Difficult & Neutral & Easy & Very Easy & Extremely Easy
\end{tabular}




\section{APPENDIX J - HAPTIC ALERT LOCATION QUESTIONNAIRE (OVERALL)}

Please answer each question by selecting a number on the scale that best reflects your response. Half numbers, such as 4.5, are also acceptable. Please write down the number on the blank response line.

1. Please rate the intensity of the vibration alerts that were presented through the bottom of the driver seat.

Response:

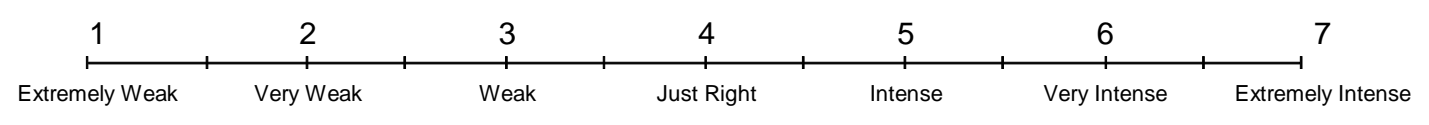

2. Please rate the intensity of the vibration alerts that were presented through the back of the driver seat

Response:

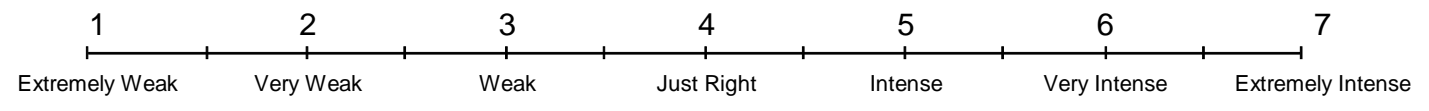

3. Please rate how annoying the vibration alerts were when presented through the bottom of the driver seat.

Response:

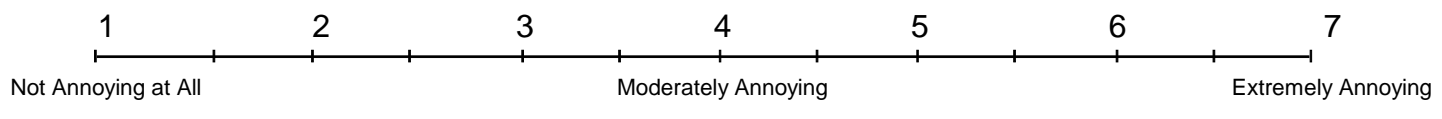

4. Please rate how annoying the vibration alerts were when presented through the back of the driver seat.

Response:

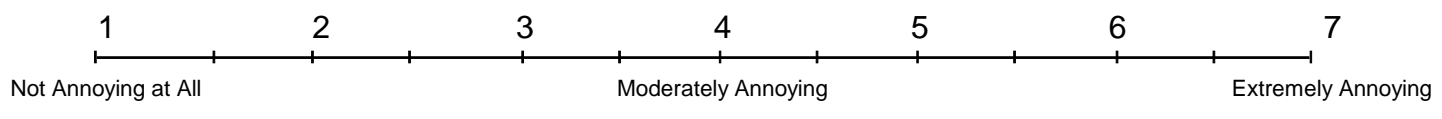


Participant \#:

5. How appropriate is it to vibrate the bottom of the driver seat to alert you to a crash threat?

Response:

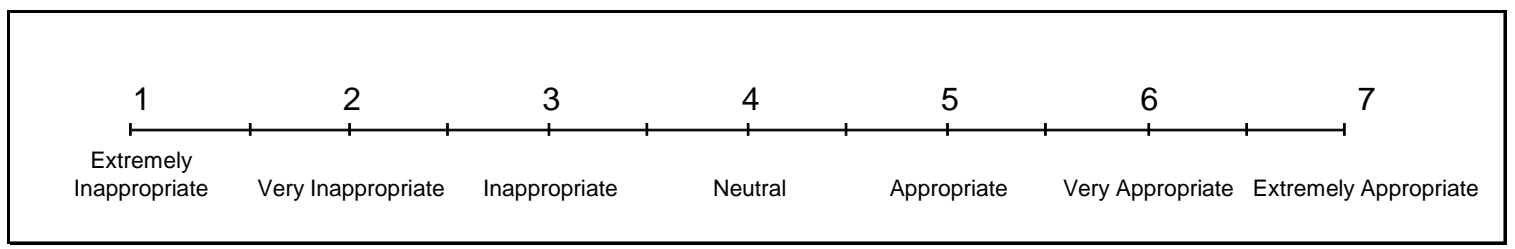

6. How appropriate is it to vibrate the back of the driver seat to alert you to a crash threat?

Response:

\begin{tabular}{|c|c|c|c|c|c|c|}
\hline 1 & 2 & 3 & 4 & 5 & 6 & 7 \\
\hline $\begin{array}{l}\text { Extremely } \\
\text { Inappropriate }\end{array}$ & Very Inappropriate & Inappropriate & Neutral & Appropriate & Very Appropriate & Extremely Appropriate \\
\hline
\end{tabular}




\section{APPENDIX K - ADDITIONAL DRIVER RATINGS OF ALERTS IN EXPERIMENT 2}

At the end of Experiment 2, participants answered the following questions.

Question 1: Please rate the intensity of the vibration alerts that were presented through the bottom of the driver seat.

On average, drivers rated the intensity of the alerts presented through the bottom of the driver seat as just right (mean response $=4.25$, s.e. $=0.15$ ). Figure 70 shows drivers' ratings broken down by age and gender.

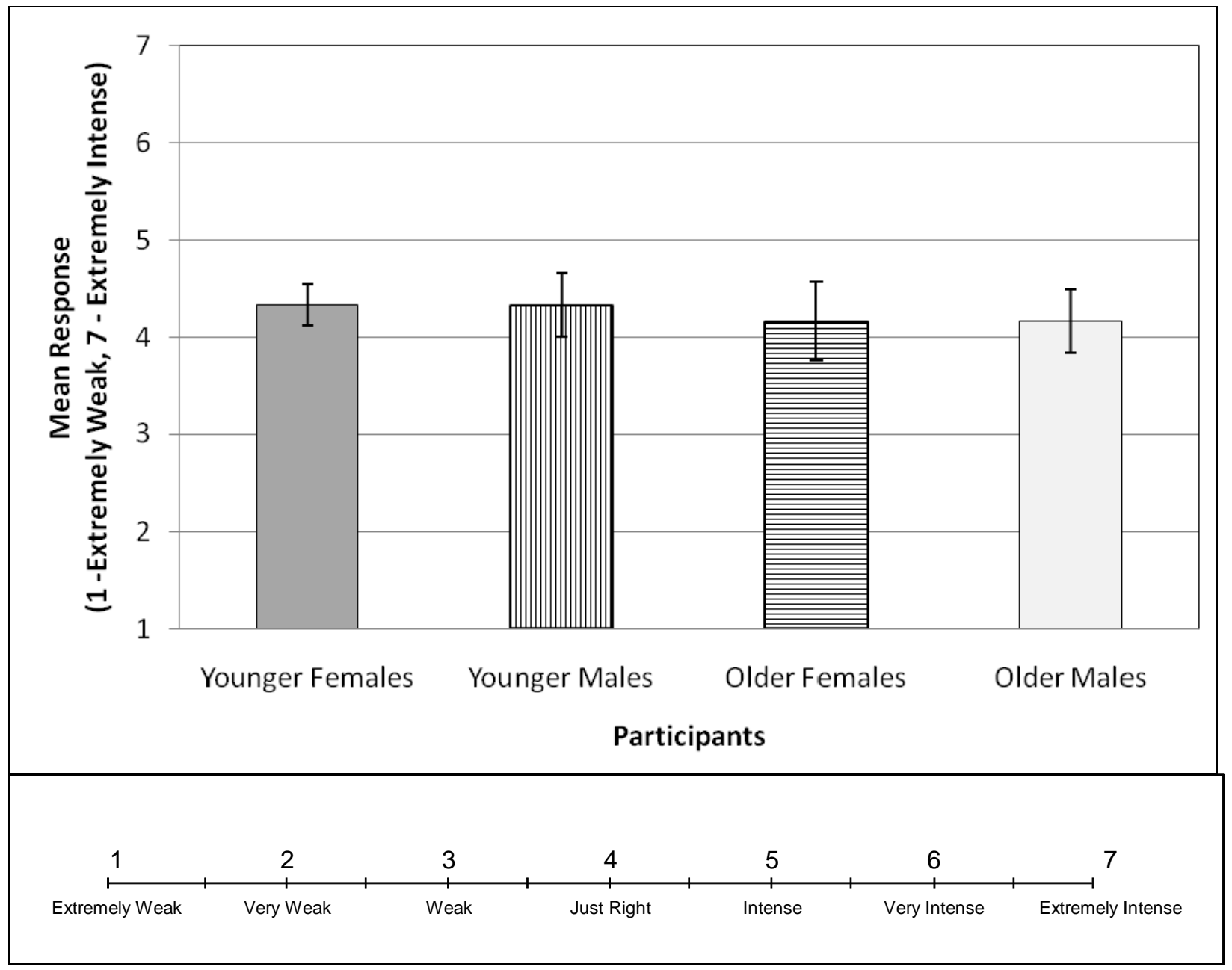

Figure 70. Drivers' mean ratings of the intensity of the vibration alerts that were presented through the seat pan. 
Question 2: Please rate the intensity of the vibration alerts that were presented through the back of the driver seat.

On average, drivers rated the intensity of the alerts presented through the back of the driver seat as intense (mean response $=4.88$, s.e. $=0.20$ ). Figure 71 shows drivers' ratings broken down by age and gender.

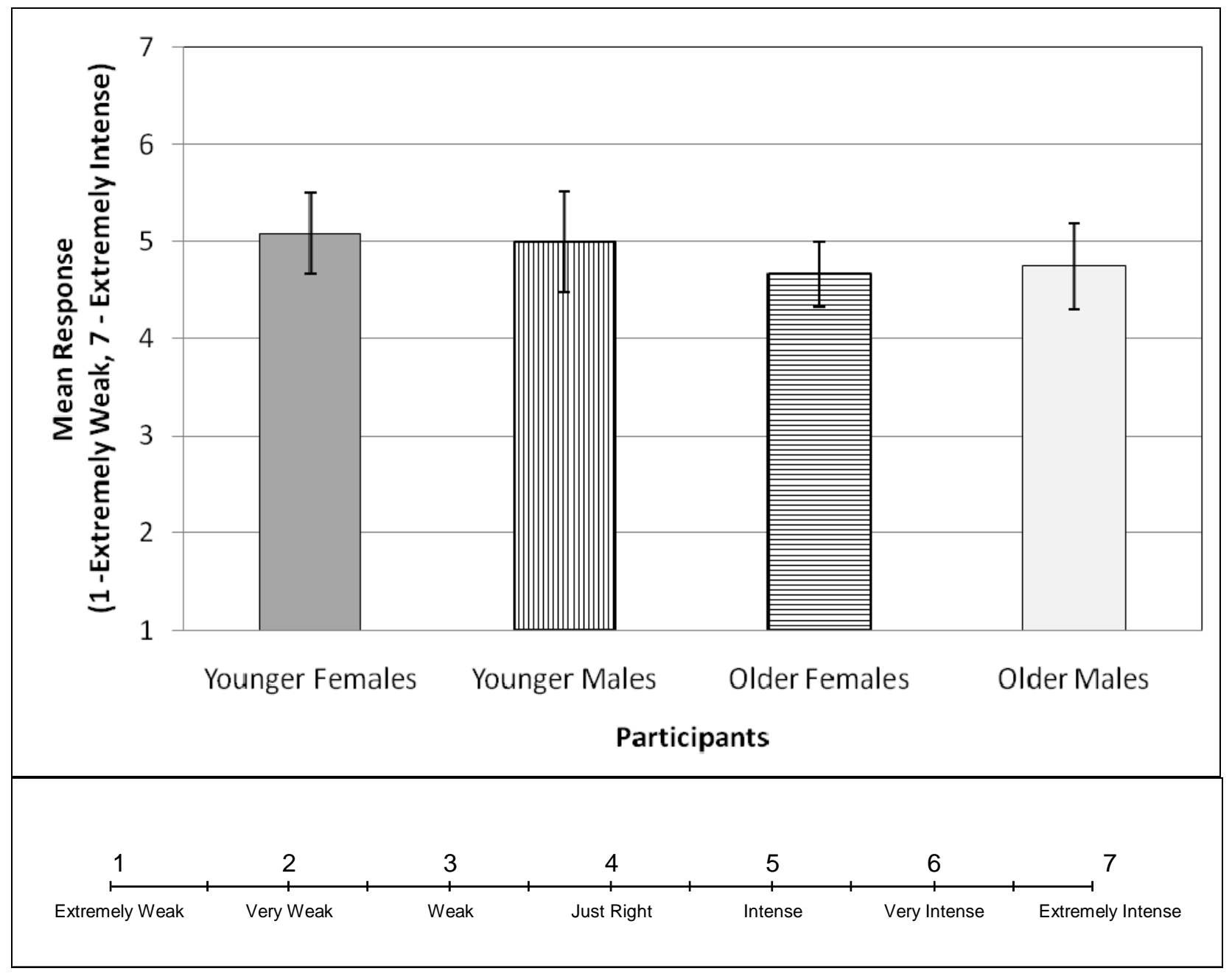

Figure 71. Drivers' mean ratings of the intensity of the vibration alerts that were presented through the seat back. 
Question 3: Please rate how annoying the vibration alerts were when presented through the bottom of the driver seat.

On average, drivers found the alerts presented through the bottom of the driver seat to be less than moderately annoying (mean response $=3.00$, s.e. $=0.25$ ). Figure 72 shows drivers' ratings broken down by age and gender.

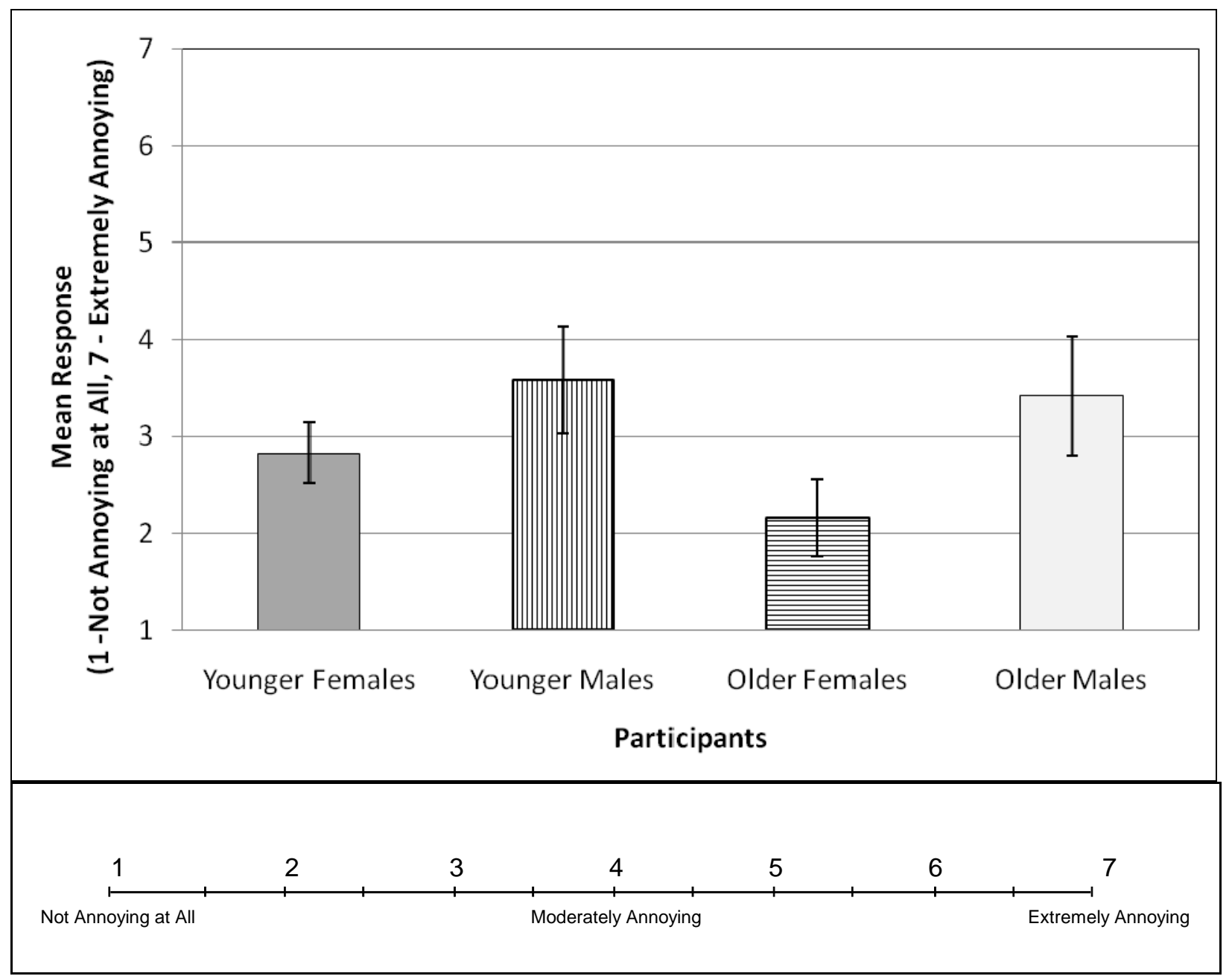

Figure 72. Drivers' mean ratings of how annoying the vibration alerts were when presented through the seat pan. 
Question 4: Please rate how annoying the vibration alerts were when presented through the back of the driver seat.

On average, drivers found the alerts presented through the back of the driver seat to be less than moderately annoying (mean response $=2.60$, s.e. $=0.27$ ). Figure 73 shows drivers' ratings broken down by age and gender.

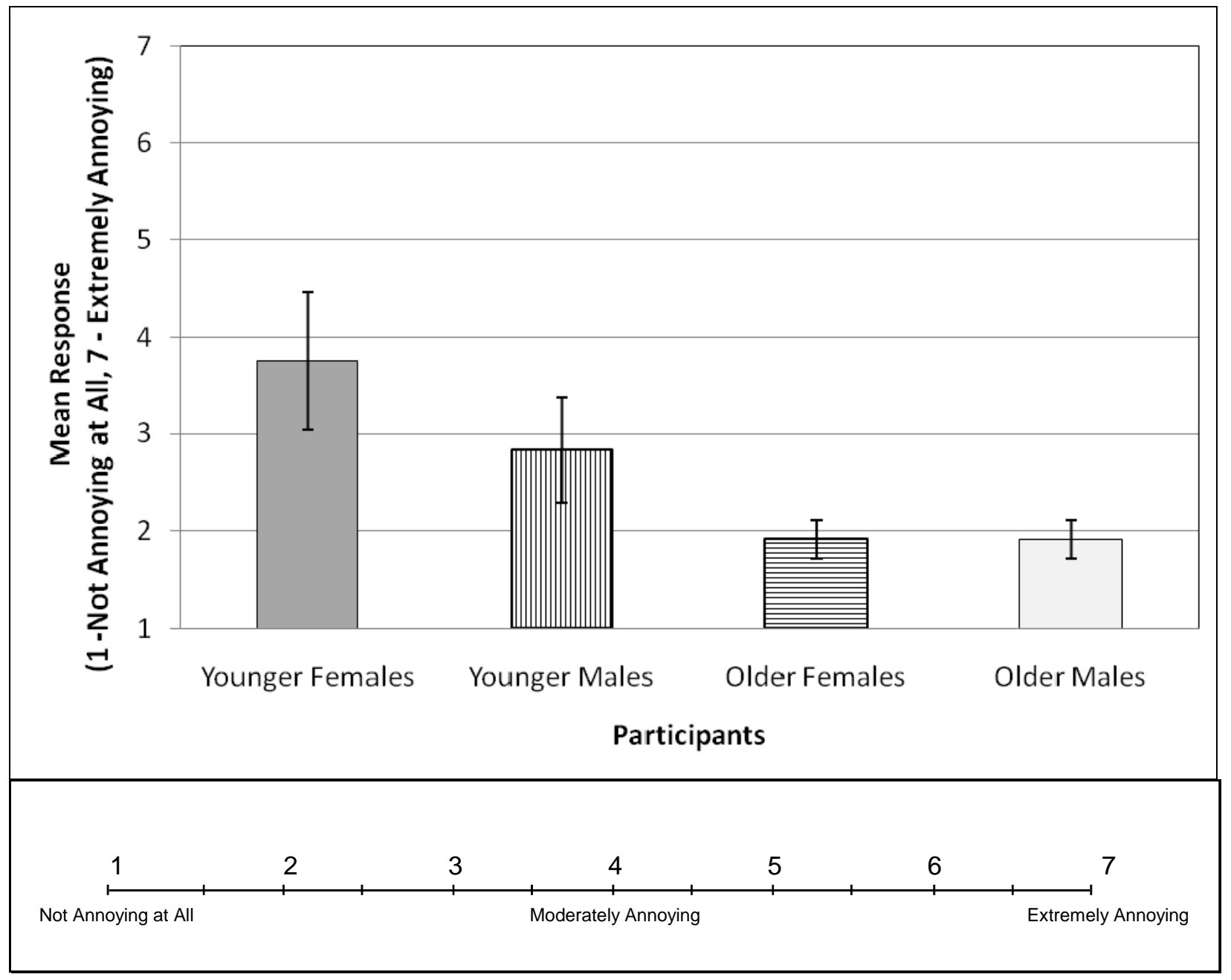

Figure 73. Drivers' mean ratings of how annoying the vibration alerts were when presented through the seat back. 
Question 4: Please rate how appropriate it is to vibrate the bottom of the driver seat to alert you to a crash threat.

On average, drivers reported that it was appropriate to vibrate the bottom of the driver seat when alerting them to a crash threat (mean response $=5.04$, s.e. $=0.26$ ). Figure 74 shows drivers' ratings broken down by age and gender.

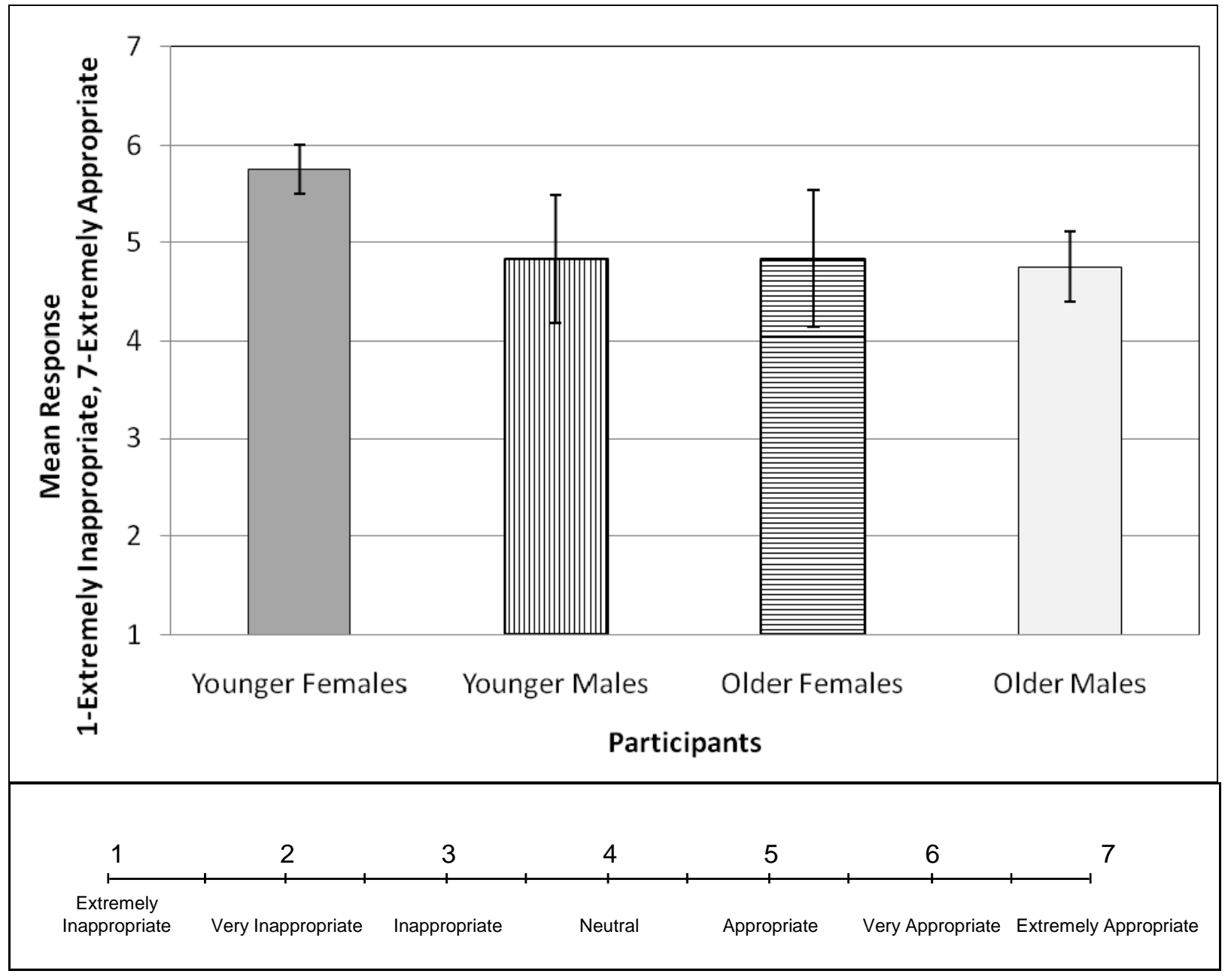

Figure 74. Drivers' mean ratings of how appropriate it is to vibrate the bottom of the driver seat to present an alert. 
Question 5: Please rate how appropriate it is to vibrate the back of the driver seat to alert you to a crash threat.

On average, drivers reported that it was very appropriate to vibrate the back of the driver seat when alerting them to a crash threat (mean response $=5.52$, s.e. $=0.22$ ). Figure 75 shows drivers' ratings broken down by age and gender.

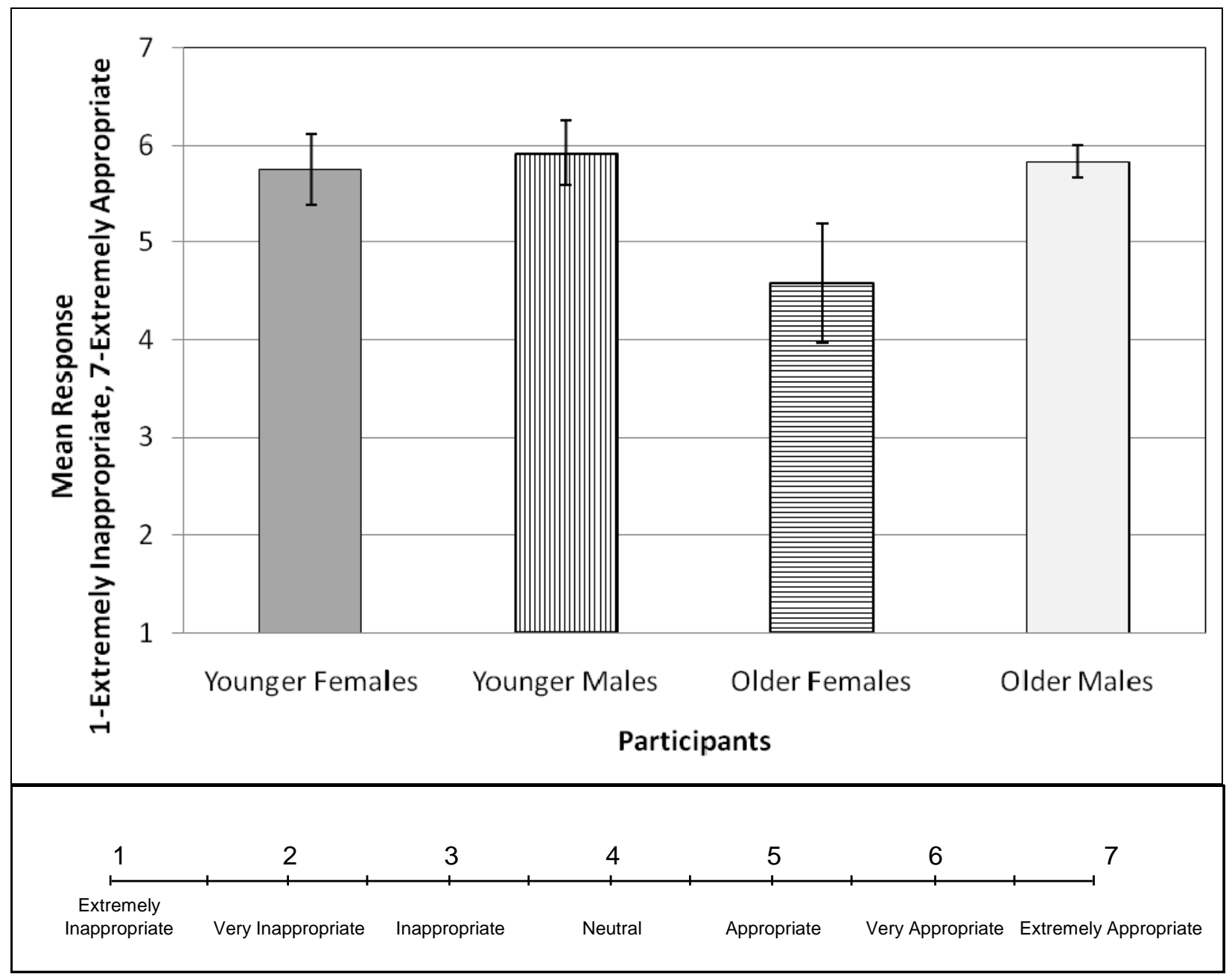

Figure 75. Drivers' mean ratings of how appropriate it is to vibrate the back of the driver seat to present an alert. 
Participant \#:

\section{APPENDIX L - SURPRISE BRAKING EVENT}

Please answer each question by selecting a number on the scale that best reflects your response. Half numbers, such as 4.5, are also acceptable. Please write down the number on the blank response line.

1. Please rate how surprised you were that you had to stop.

Response:

\begin{tabular}{|c|c|c|c|c|c|c|}
\hline 1 & 2 & 3 & 4 & 5 & 6 & 7 \\
\hline $\begin{array}{l}\text { Extremely } \\
\text { Unsurprised }\end{array}$ & Very Unsurprised & Unsurprised & Neutral & Surprised & Very Surprised & $\begin{array}{l}\text { Extremely } \\
\text { Surprised }\end{array}$ \\
\hline
\end{tabular}

2. Please rate how distracted you were while the barricade was inflated.

Response:

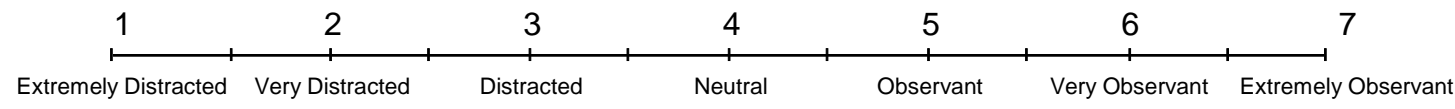

3. Please rate how much this event felt like an actual emergency braking event.

Response:

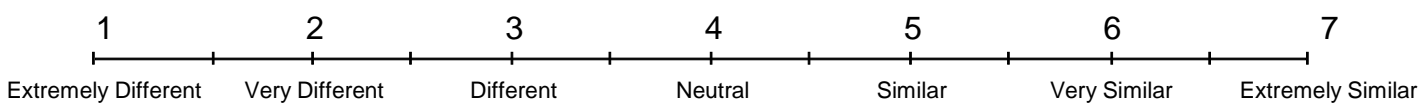

4. Please rate how fast you were at pressing the brakes.

Response:

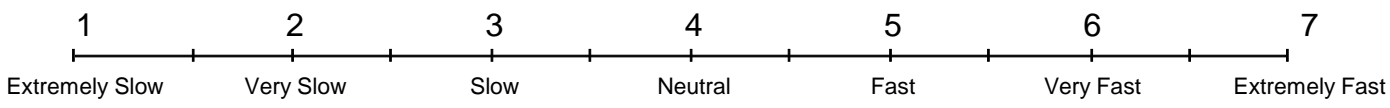

Check here if you did not press on the brakes: 
Participant \#:

5. Please rate how hard you pressed on the brakes - extremely hard braking being what you would perform in response to a child darting out in front of your car.

Response:

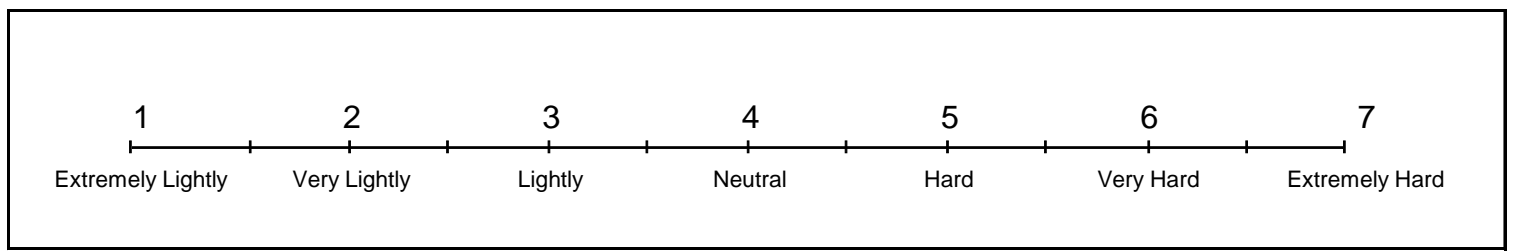

Check here if you did not press on the brakes:

6. Was there anything that prevented you from braking hard? If so, please explain? 
Participant \#:

\section{APPENDIX M - SURPRISE BRAKING WITH HAPTIC SEAT}

Please answer each question by selecting a number on the scale that best reflects your response. Half numbers, such as 4.5, are also acceptable. Please write down the number on the blank response line.

1. Please rate how surprised you were that you had to stop.

Response:

$\begin{array}{cccccc}\begin{array}{c}\text { Extremely } \\ \text { Unsurprised }\end{array} & \text { Very Unsurprised } & \text { Unsurprised } & \text { Neutral } & \text { Surprised } & \text { Very Surprised } \\ \begin{array}{c}\text { Extremely } \\ \text { Surprised }\end{array}\end{array}$

2. Please rate how distracted you were while the barricade was inflated.

Response:

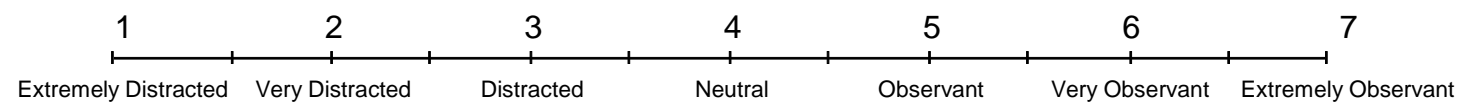

3. Please rate how much this event felt like an actual emergency braking event.

Response:

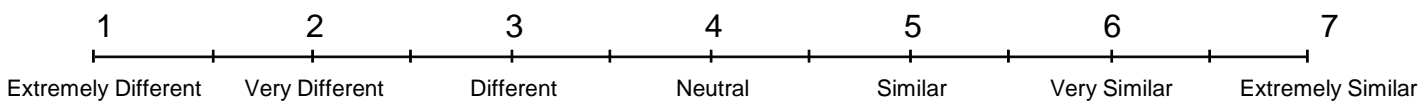

4. Please rate how well the vibrating seat grabbed your attention.

Response:

$\begin{array}{llccccc}1 & 2 & 3 & 4 & 5 & 6 & 7 \\ \begin{array}{l}\text { Extremely } \\ \text { Ineffective }\end{array} & \text { Very Ineffective } & \text { Ineffective } & \text { Neutral } & \text { Effective } & \text { Very Effective } & \begin{array}{c}\text { Extremely } \\ \text { Effective }\end{array} \\ & & & & \end{array}$


Participant \#:

5. Please rate how appropriate the vibration alert was for telling you to look forward.

Response:

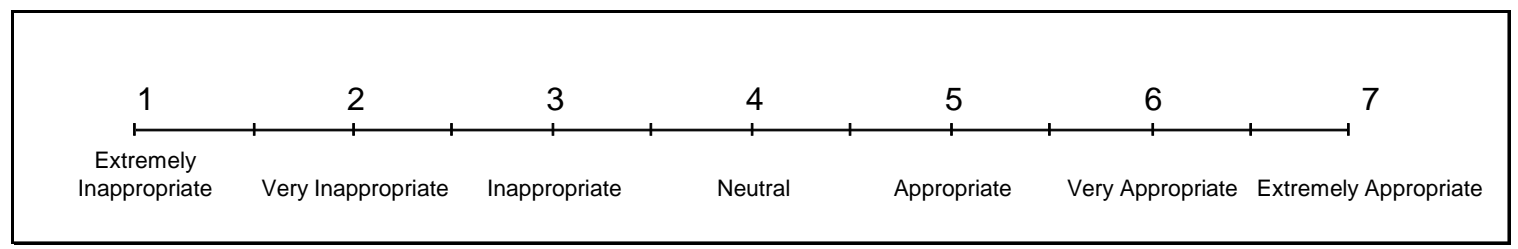

6. Please indicate the degree to which the vibration made you look forward.

I didn't look up at all

I looked up, but it had nothing to do with the vibration

I looked up, but only after hesitating due to confusion

I looked up immediately in response to the vibration

7. Please rate how fast you were at pressing the brakes.

Response:

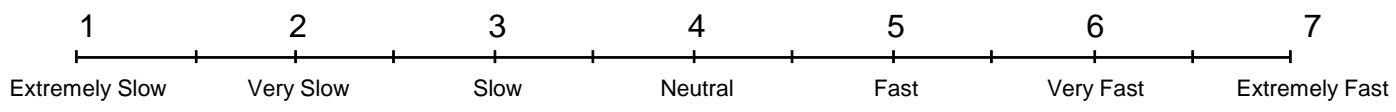

Check here if you did not press on the brakes: 
8. Please rate how hard you pressed on the brakes - extremely hard braking being what you would perform in response to a child darting out in front of your car.

Response:

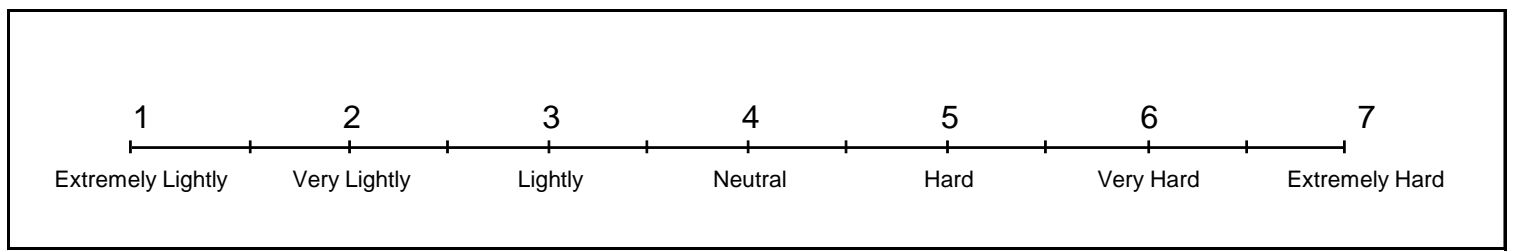

Check here if you did not press on the brakes:

7. Was there anything that prevented you from braking hard? If so, please explain?

8. Please rate how much you agree with the following statement "If my car could detect a collision before it occurs, I want my driver seat to vibrate in this manner to warn me."

Response:

$\begin{array}{ccccccc}1 & 2 & 3 & 4 & 5 & 6 \\ \text { Strongly Disagree } & \text { Somewhat Disagree } & \text { Disagree } & \text { Neutral } & \text { Agree } & \text { Somewhat Agree } & \text { Strongly Agree }\end{array}$




\section{APPENDIX N - SCATTER PLOTS OF THE VARIABLES ANALYZED IN EXPERIMENT 3}

This appendix presents scatter plots of the variables analyzed in Experiment 3 according to FCW alert activation. Data points associated with trials in which drivers collided with the barricade are denoted by an asterix, while all other data points are denoted by squares.

\section{Time from Inflatable Barricade Activation to Returning Eyes to the Forward Roadway}

Figure 76 separates drivers' elapsed time from the inflatable barricade being launched to them returning their gaze to the forward roadway according to those that received an FCW alert and those that did not. It can be seen that there is a tighter distribution for the group that had the FCW alert compared to the group that did not.

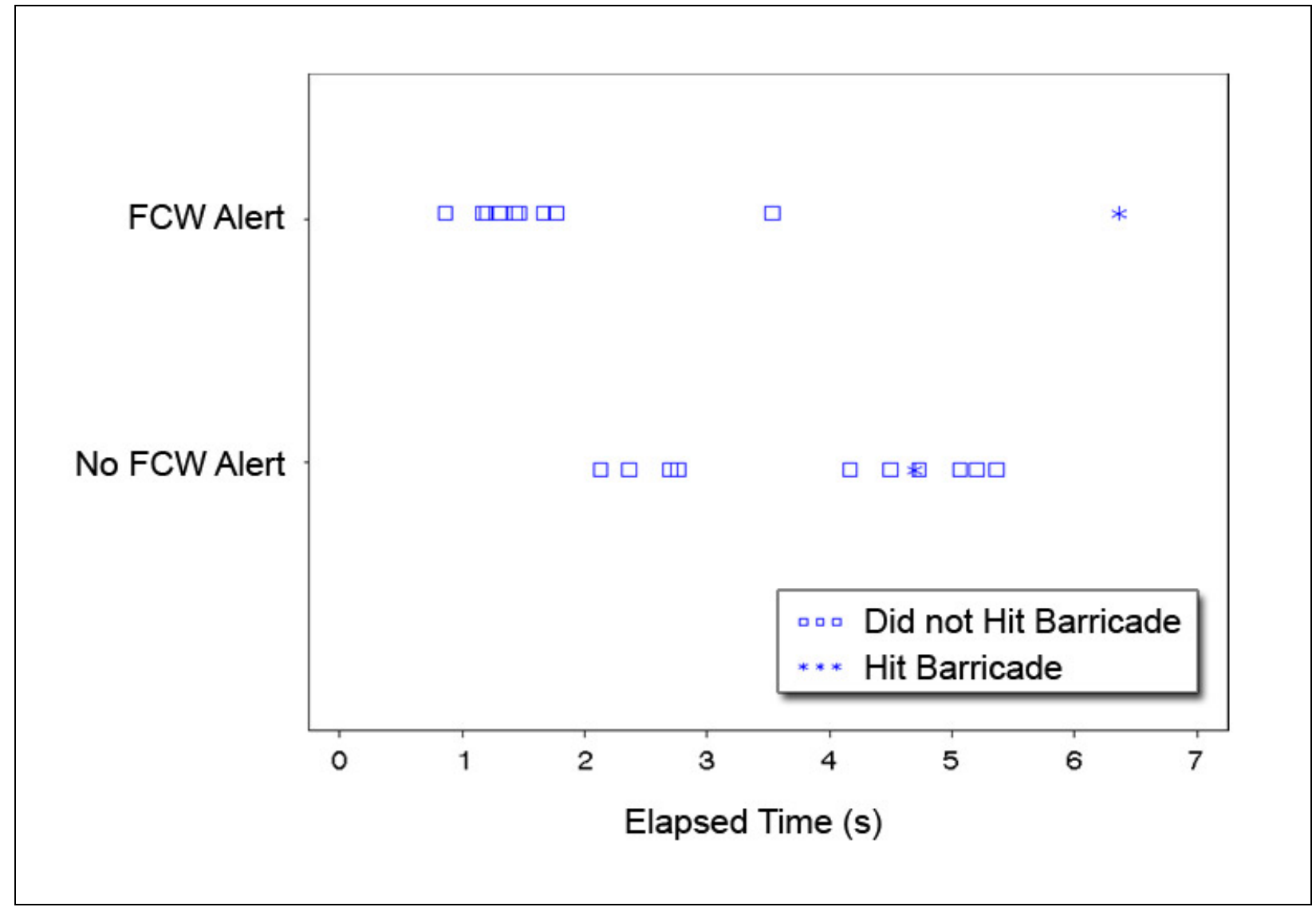

Figure 76. Time from inflatable barricade activation to returning eyes to the forward roadway. 


\section{Foot-Off Throttle Response Time}

Figure 77 separates drivers' elapsed time from the inflatable barricade being launched to them taking their foot off the throttle according to those that received an FCW alert and those that did not. It can be seen that there is a tighter distribution for the group that had the FCW alert compared to the group that did not.

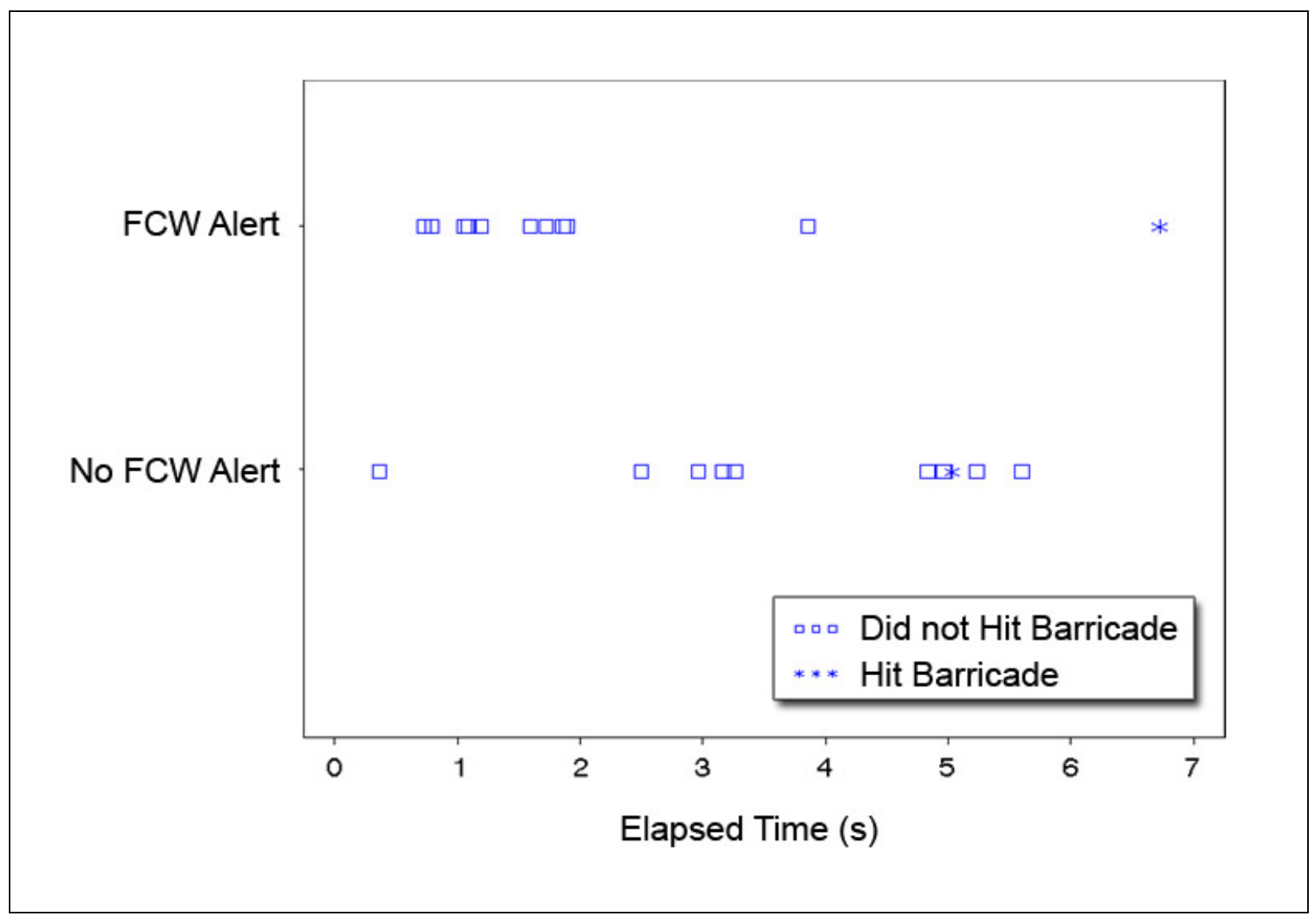

Figure 77. Foot-off throttle response time. 


\section{Foot-On Brake Response Time}

Figure 78 separates drivers' elapsed time from the inflatable barricade being launched to them pressing the brake pedal according to those that received an FCW alert and those that did not. It can be seen that there is a tighter distribution for the group that had the FCW alert compared to the group that did not.

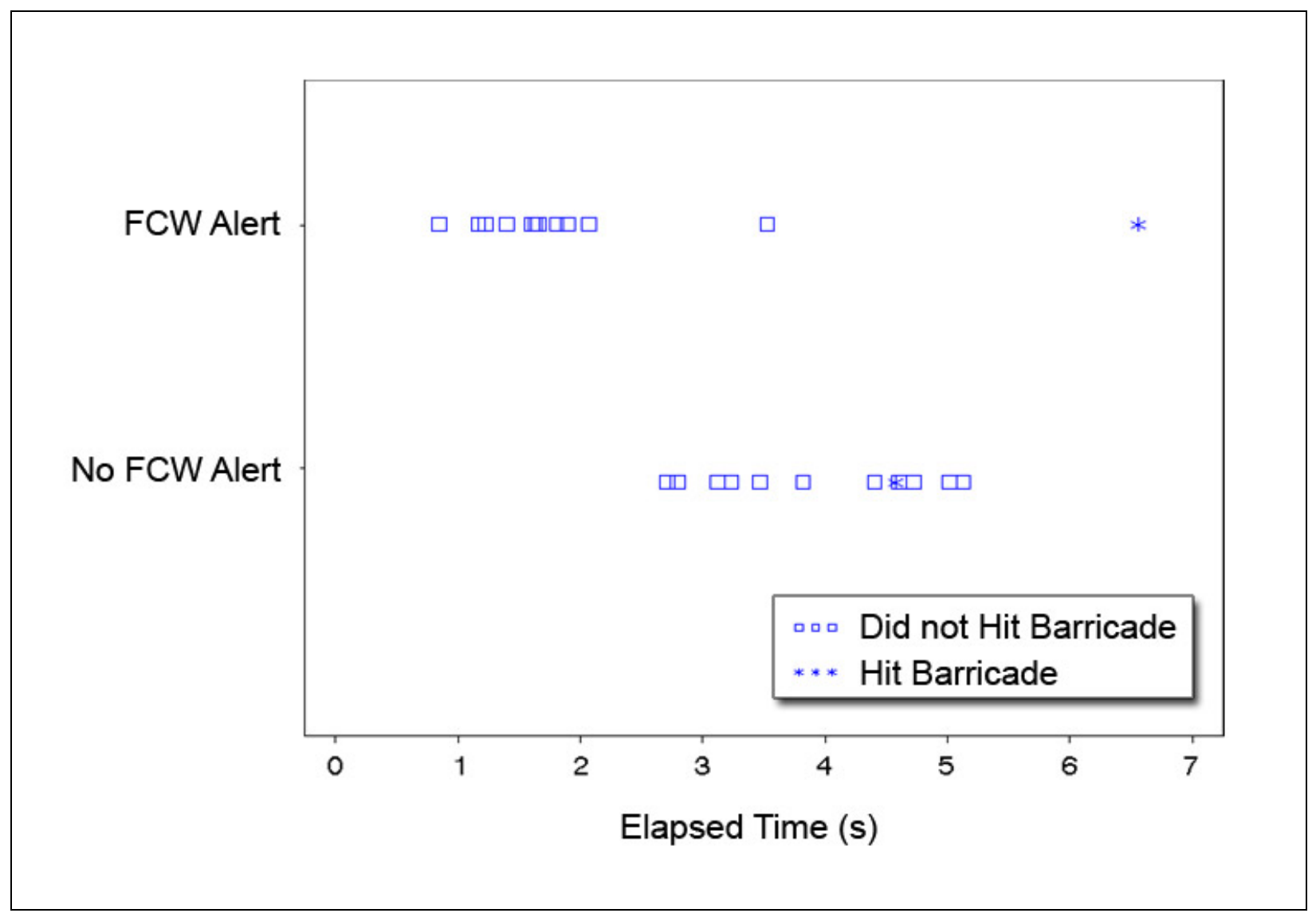

Figure 78. Foot-on brake response time. 


\section{Time Taken to Stop}

Figure 79 separates drivers' elapsed time to stop according to those that received an FCW alert and those that did not. It can be seen that there is a tighter distribution for the group that had the FCW alert compared to the group that did not.

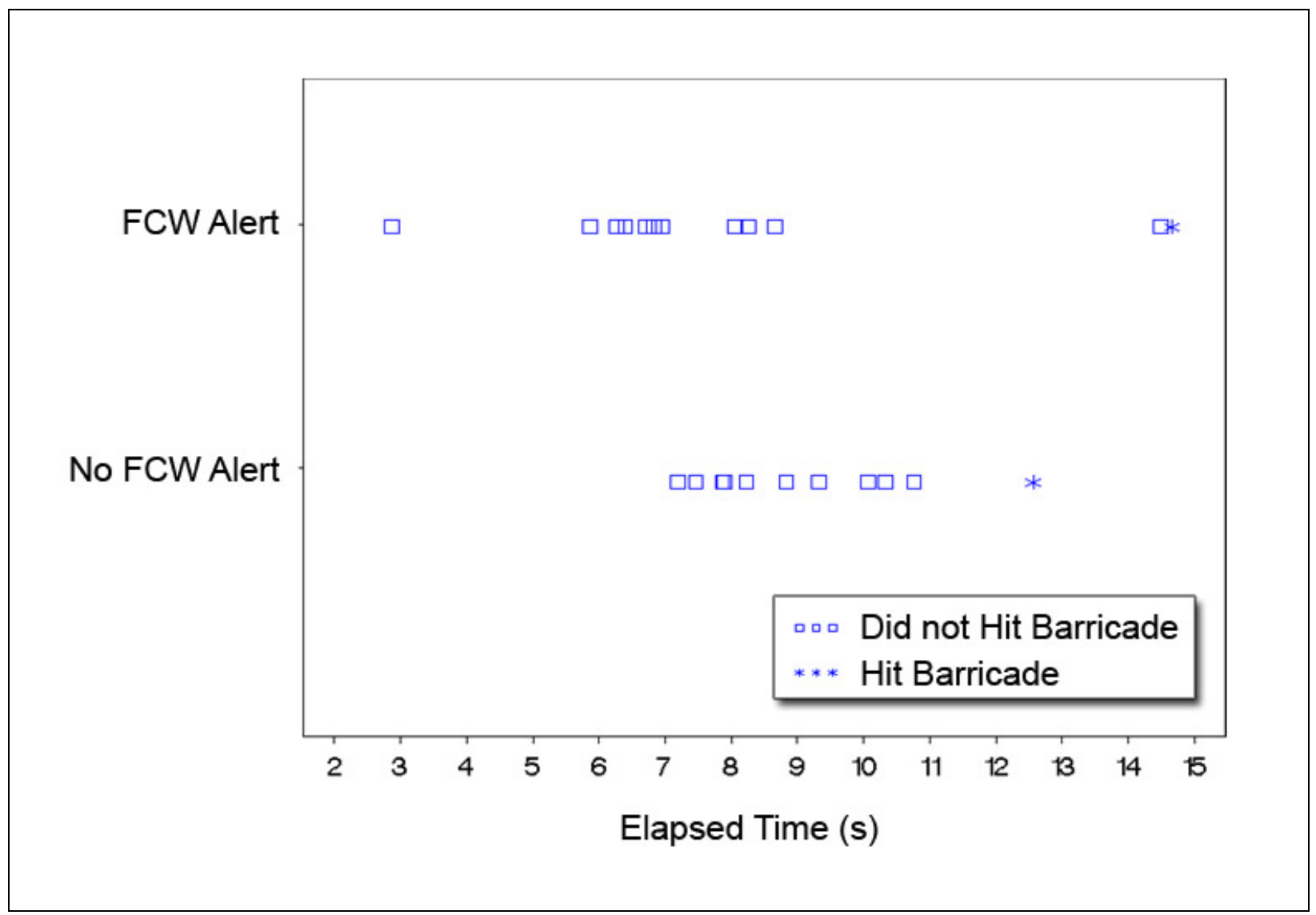

Figure 79. Time taken to stop. 


\section{Peak Deceleration}

Figure 80 separates drivers' peak deceleration according to those that received an FCW alert and those that did not. It can be seen that there is a tighter distribution for the group that had the FCW alert compared to the group that did not.

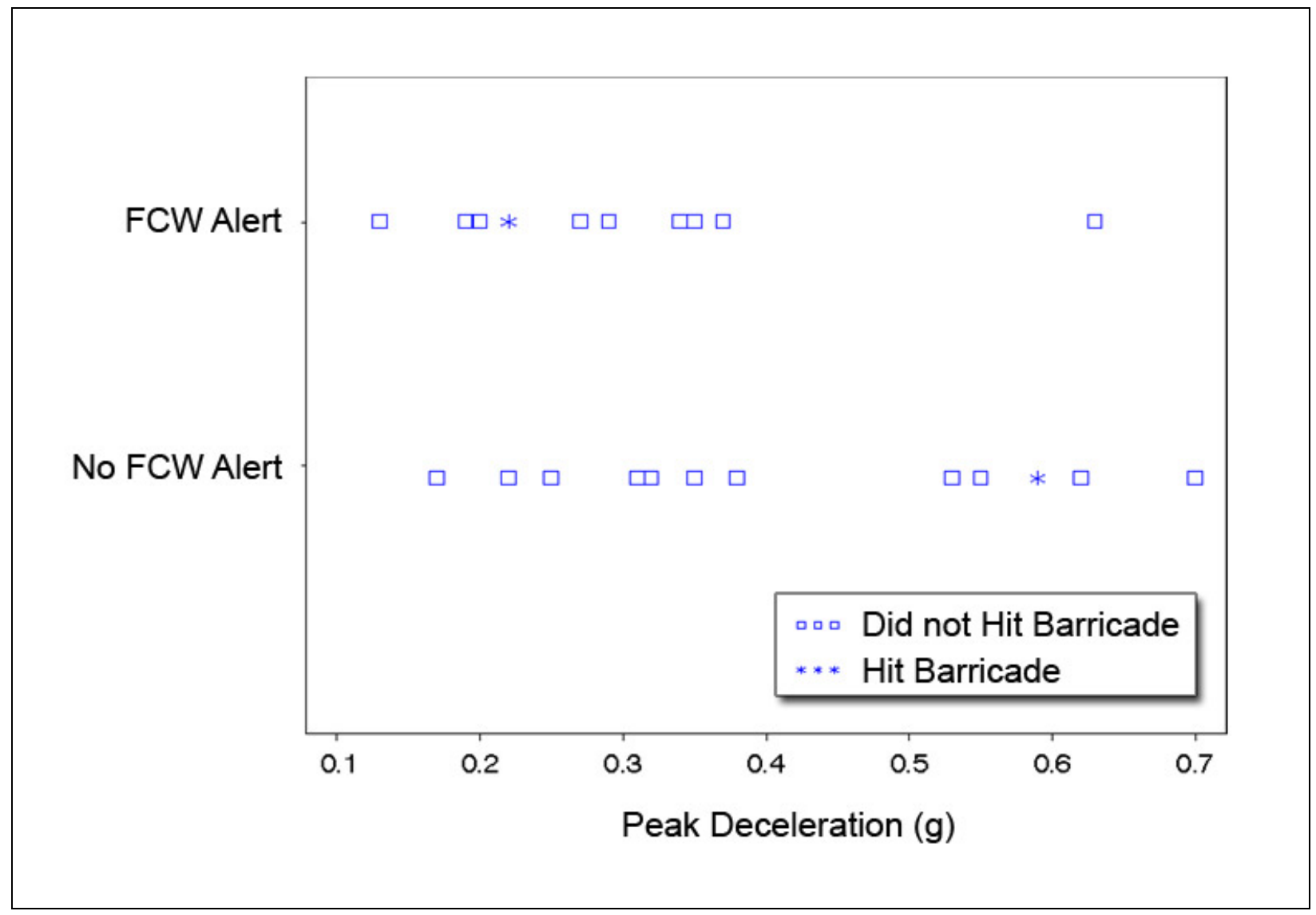

Figure 80. Peak deceleration. 


\section{Distance Between Car and Inflatable Barricade}

Figure 81 separates drivers' peak deceleration according to those that received an FCW alert and those that did not. It can be seen that there is a tighter distribution for the group that had the FCW alert compared to the group that did not.

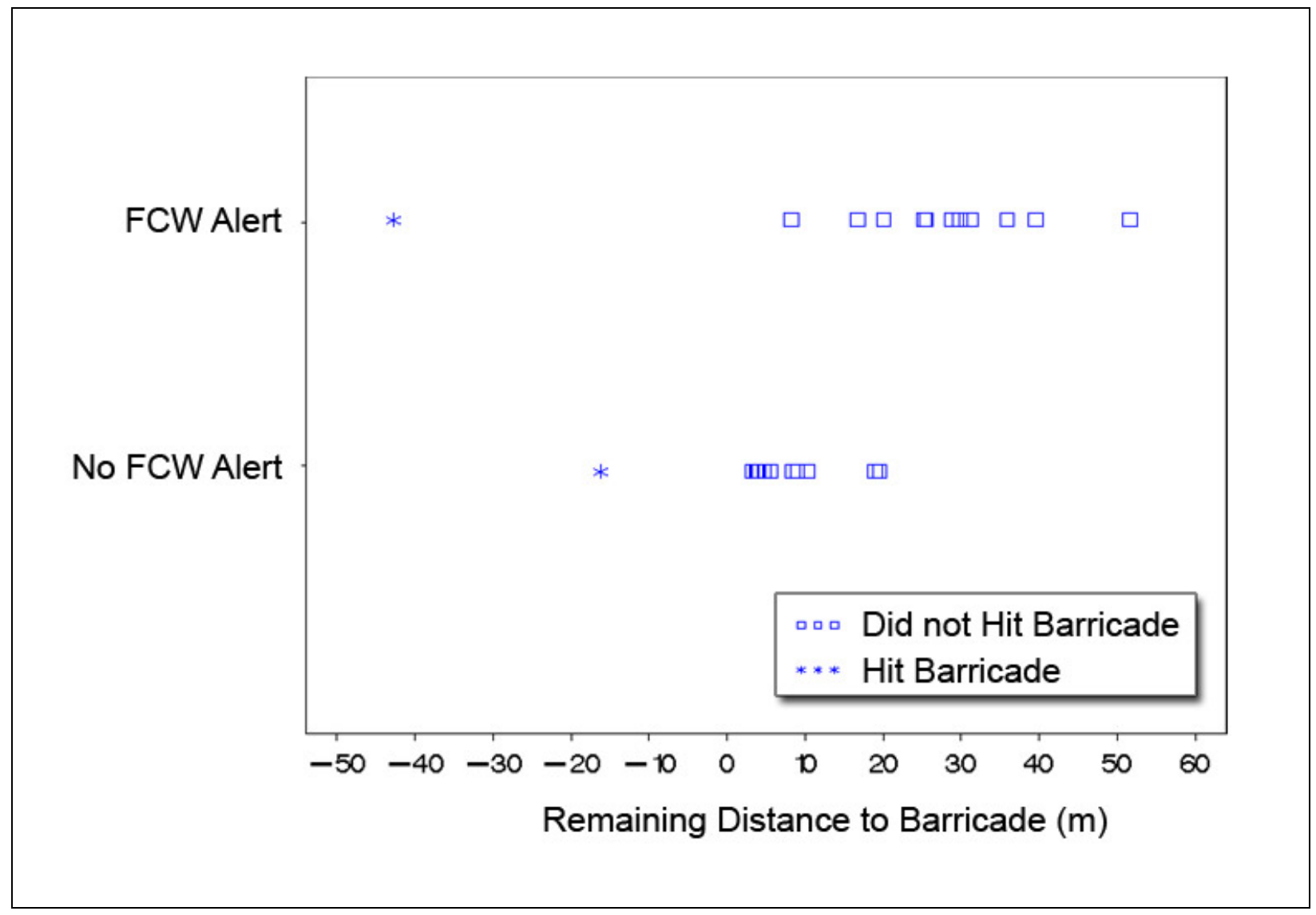

Figure 81. Remaining distance between vehicle and inflatable barricade. 


\section{APPENDIX O - EXPERIMENT 3 DRIVER RATINGS}

Question 1: Please rate how much this event felt like an actual emergency braking event.

On average, drivers that did not receive an FCW alert reported that the surprise event felt similar to an actual emergency braking event (mean response $=5.08$, s.e. $=0.45$ ). Drivers that received an FCW alert also reported that the event felt similar to an emergency braking event (mean response $=5.50$, s.e. $=0.19)$. A $2($ Age $) \times 2($ Gender $) \times 2($ FCW Alert $)$ between-subjects ANOVA was performed on drivers' ratings. No significant differences were found. Figure 82 shows drivers' ratings broken down by age and gender.

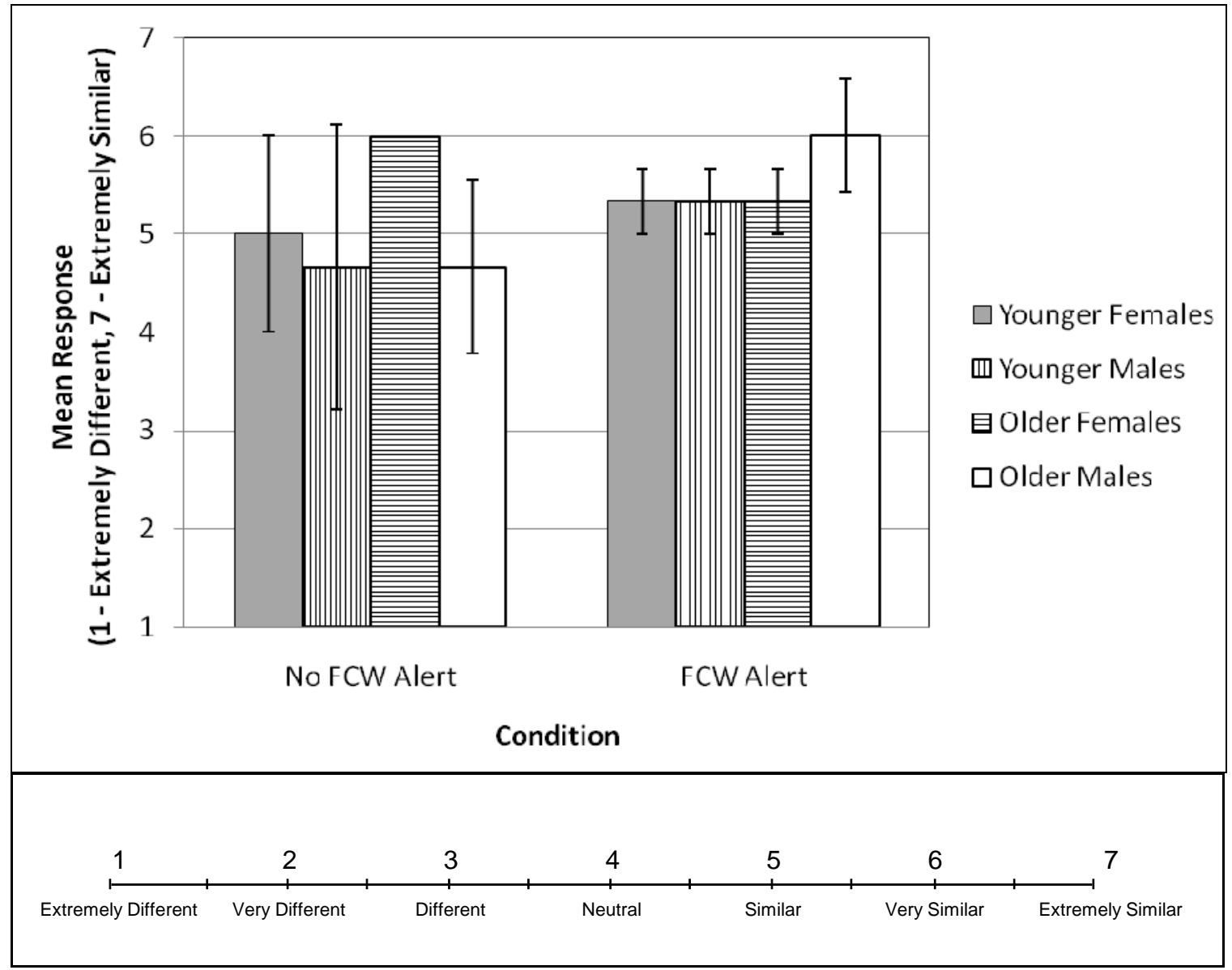

Figure 82. Drivers' rating of event's similarity to an actual emergency braking event. 
Question 2: Please rate how fast you were at pressing the brakes.

On average, drivers that did not receive an FCW alert reported that they were fast at pressing the brakes (mean response $=4.54$, s.e. $=0.37$ ). Drivers that received an FCW alert were neutral when asked how fast they were at pressing the brakes (mean response $=4.42$, s.e. $=0.36$ ). A 2 (Age) x 2 (Gender) x 2 (FCW Alert) between-subjects ANOVA was performed on drivers' ratings of how fast they were at pressing the brakes. A significant gender effect was found $(\mathrm{F}(1$, $16)=5.45, \mathrm{p}=0.0329)$. Females rated themselves to have pressed the brakes "Fast" (mean = 4.95 , s.e. $=0.32$ ), while males were neutral in their ratings of how fast they pressed the brakes $($ mean $=4.00$, s.e. $=0.35)$. An Age $x$ Gender $\times F C W$ Alert interaction was also found $(F(1,16)=$ $8.67, \mathrm{p}=0.0095)$. Figure 83 shows drivers' ratings broken down by age and gender.

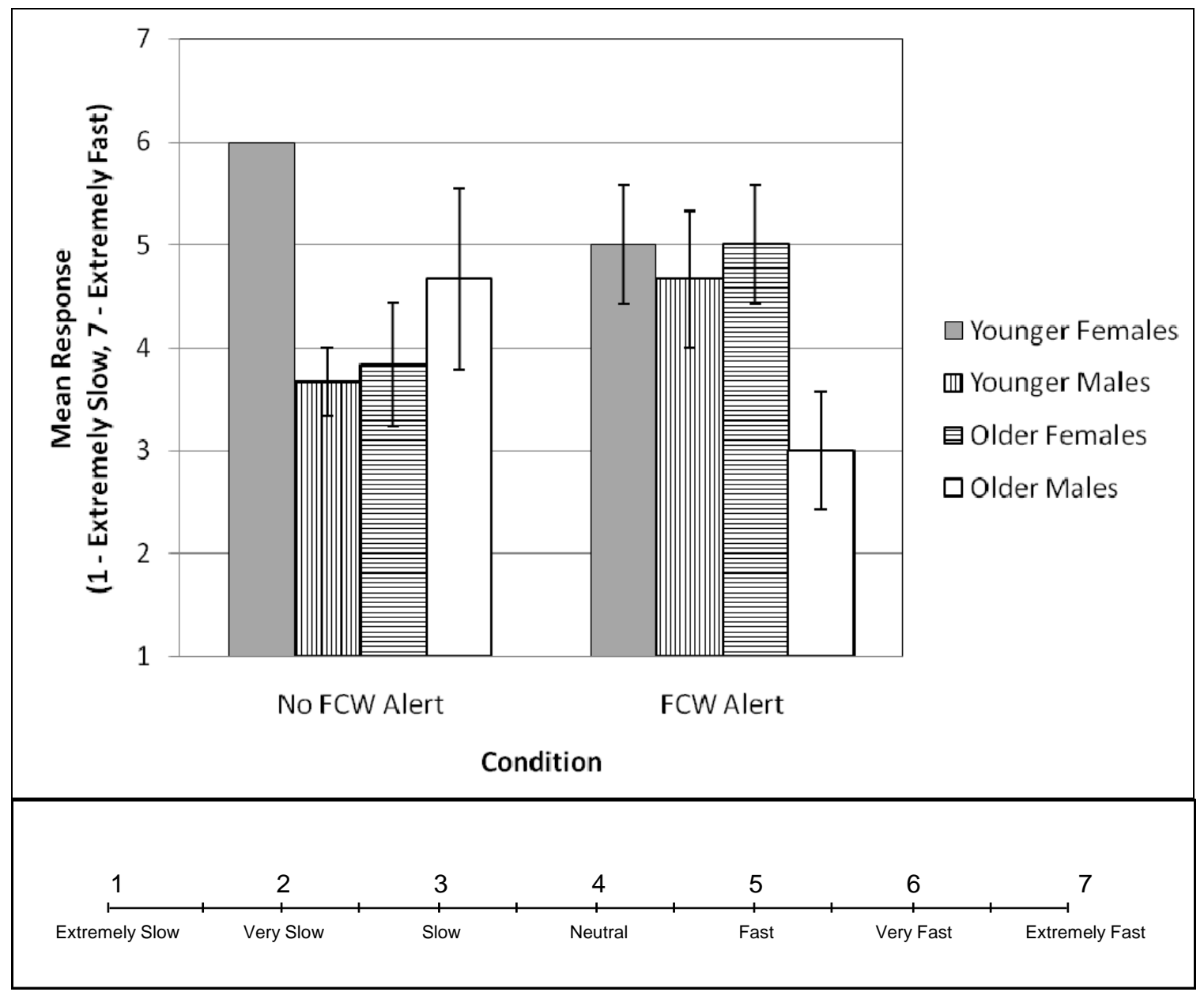

Figure 83. Drivers' ratings of how fast they pressed the brakes broken down by age and gender. 
Question 3: Please rate how hard you pressed on the brakes - extremely hard braking being what you would perform in response to a child darting out in front of your car.

On average, drivers that did not receive an FCW alert reported that they pressed hard on the brakes (mean response $=4.71$, s.e. $=0.30$ ). Drivers that received an FCW alert were neutral when asked how hard they pressed on the brakes (mean response $=4.17$, s.e. $=0.30$ ). A 2 (Age) x 2 (Gender) x 2 (FCW Alert) between-subjects ANOVA was performed on drivers' ratings of how hard they pressed on the brakes. No significant differences were found. Figure 84 shows drivers' ratings broken down by age and gender.

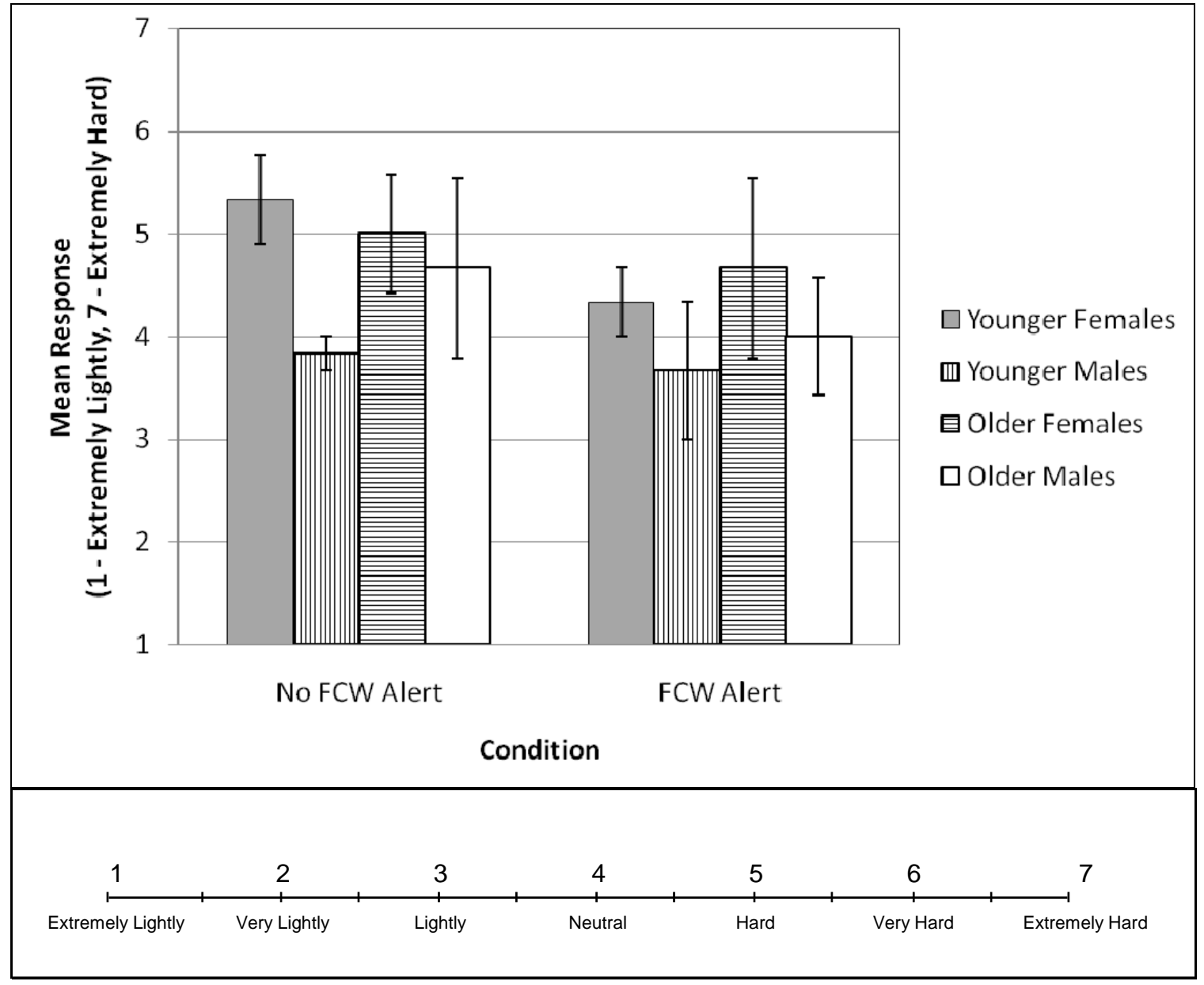

Figure 84. Drivers' ratings of how hard they pressed on the brakes broken down by age and gender. 
Question 4: Please rate how well did the vibrating seat grab your attention.

On average, the drivers that received the FCW alert while they performed the search task reported that it was effective at grabbing their attention (mean response $=4.75$, s.e. $=0.55$ ). Figure 85 shows drivers' ratings broken down by age and gender.

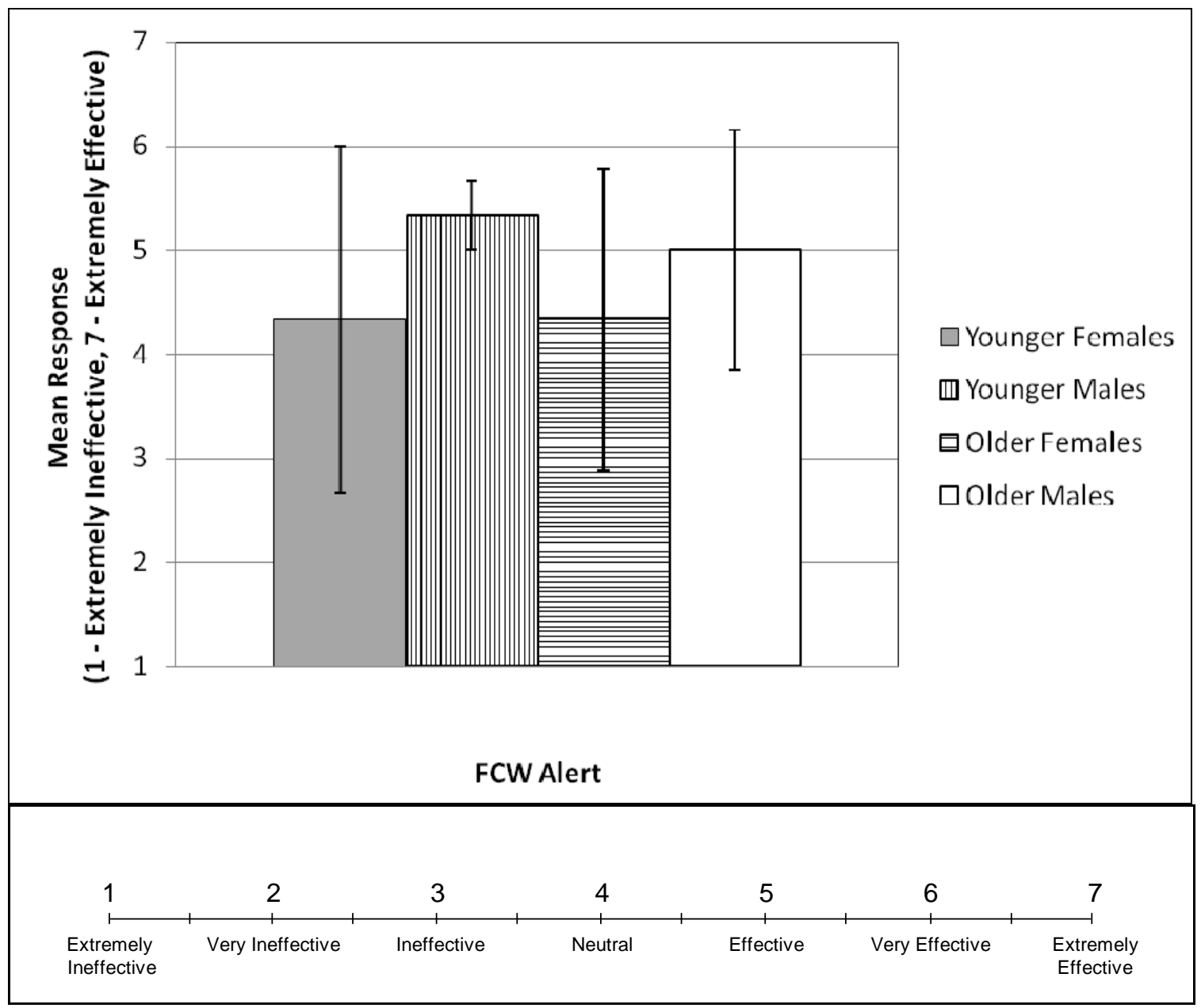

Figure 85. Drivers' ratings of how well the vibrating seat grabbed their attention broken down by age and gender. 
Question 5: Please rate how appropriate the vibration alert was for telling you to look forward.

On average, the drivers that received the FCW alert while they performed the search task reported that it was appropriate for telling them to look forward (mean response $=4.50$, s.e. $=$ 0.60 ). Figure 86 shows drivers' ratings broken down by age and gender.

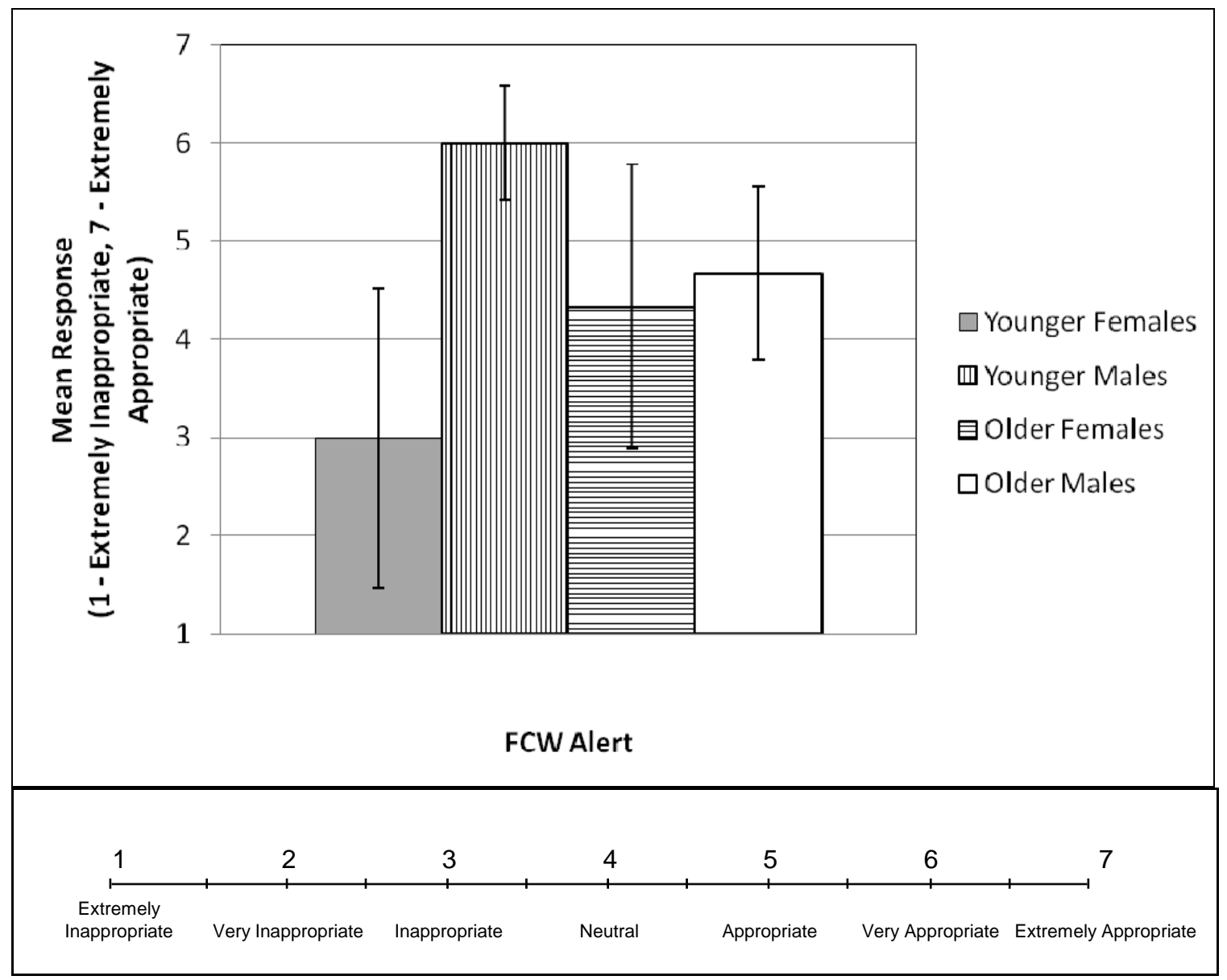

Figure 86. Drivers' ratings of how appropriate the vibrating seat was for telling them to look forward broken down by age and gender. 
Question 6: Please rate how much you agree with the following statement "If my car could detect a collision before it occurs, I want my driver seat to vibrate in this manner to warn me."

On average, drivers reported that they agreed with the statement "If my car could detect a collision before it occurs, I want my driver seat to vibrate in this manner to warn me" (mean response $=5.67$, s.e. $=0.43$ ). Figure 87 shows drivers' ratings broken down by age and gender.

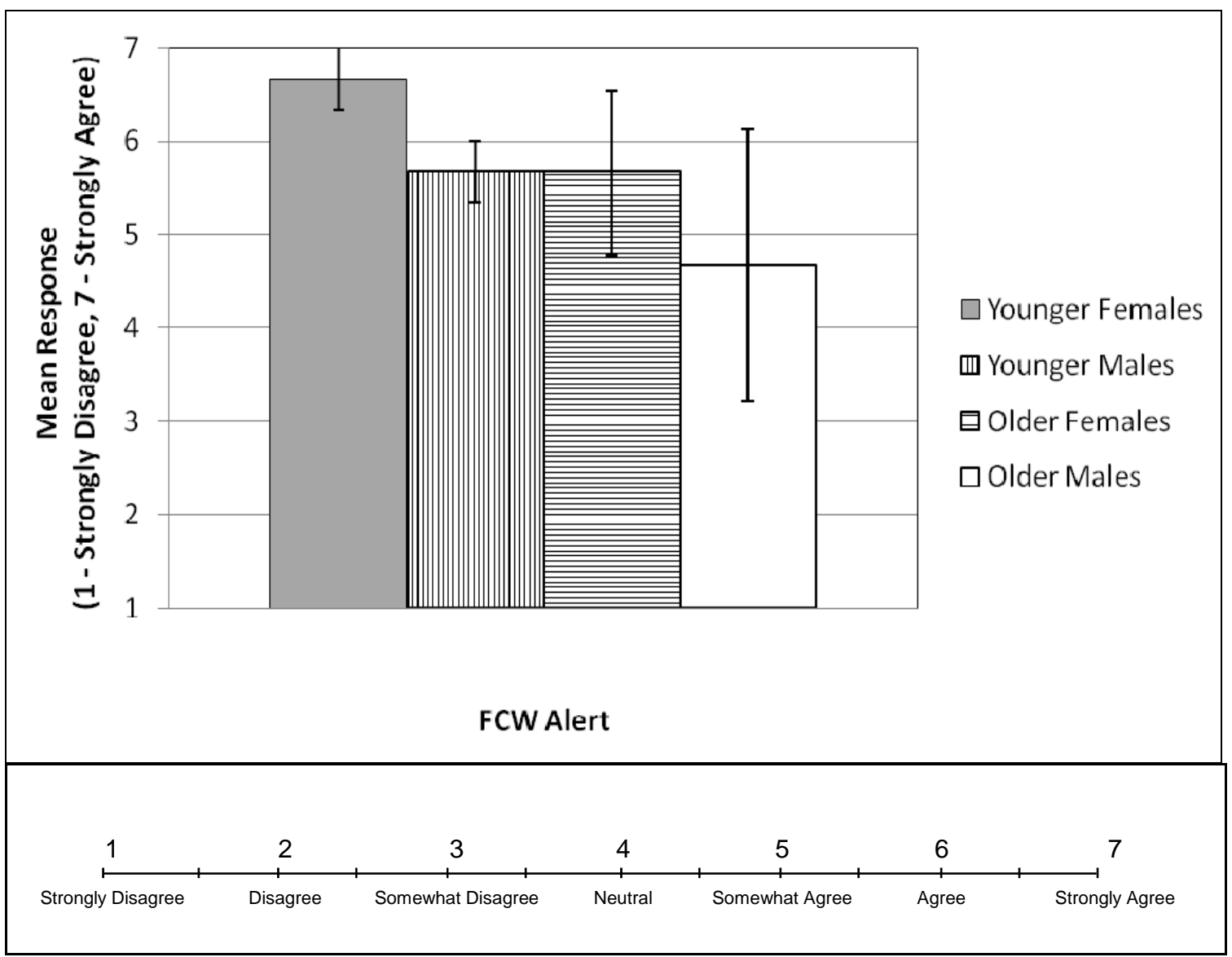

Figure 87. Drivers' ratings of how much they would like their driver seat to vibrate to warn them of an impending collision broken down by age and gender. 